II.

\title{
LISTE ALPHABETIQUE DES MEMBRES ALPHABETICAL LIST OF MEMBERS
}

Cette 1iste est préparée d'une bande magnétique tenu par D. Reidel Publishing Company et soutenu en comparaison des archives du Secrétariat de 1'UAI.

Les noms figurent à la première lettre du premier mot; par exemple, le nom De Jong se trouve à la lettre D.

Les noms des pays sont données sous leur forme anglaise.

Les membres de l'Union sont priês de bien vouloir informer le Secrétaire de 1'UAI, 61 ave: de l'Observatoire, F-75014 Paris, France, de toutes les erreurs qu'ils pourraient relever dans la liste.

This list is prepared from a magnetic tape held by D. Reidel Publishing Company and collated with the records of the IAU Secretariat.

Names are given under the first letter of the first word; for example, the name De Jong is to be found under $D$.

The names of countries are given in the English form.

Please inform the Secretary of the IAU at 61 ave. de 1 'Observatoire, F-75014 Paris, France, of any errors that you may find in this 1 ist. 
A'HEAFA MICHAEL F DR ASTRE UCNY PROGRAN UAIV OF MARYLANC CELLEGE PK MO 2074 ? U.S.A.

$\triangle P A L A K I N V I C I$ IIR K CR IAST ITUTE FOR THECRE i. KLTUIZTU QUAY $1921 \times 7$ LENINGRAL U.S.S.R.

AEL ALA J IR

FVIFICINO ARAGUANEY

FLAATA RAJA NC 1

CHACAITC CARACAS 106 $\checkmark E A E L U E L A$

AHETTI O PROF

IST ARL

LARCO ENR I CO FFRMI 7

I -5 III25 FIREN?E

ITALY

APLES HAROLD J G CR

CSIRC

iIV CF RAOIOPHYSICS

$P$ C DOX 70

EPING NSW 2121

APRAHAN ZULEMA TR

CRAAN / CN/ CNPO

RUA PARA 277

$\because 12435 A 0$ PAULO SP

IRAZIL

APREL CE RRITO E J C OR

CESERVATORIOAASTRO

TAPADA

LISFCN

PCRTURAL

ACKER AGPES DR

CFSERVATOIRE

iI RUE CE

LUAIVFRSITE

F- $673 O$ STRASBOURC

FRANCE

ALANS A N MR

$6549 \mathrm{~N} 35 \mathrm{TH}$ ROAD

$\triangle K L I N G T C N$ VA 22213

U.S. $\Lambda$.

ACEL ARTHUR F PROF IMER

OCRTHEPN ARIZUNA

UNIVERSITY

FCX 5679

FLAGSTAFF AZ 86001

U.S.A.

ALCLFSSCN TORD DR

KKAGEMDLMSGATAN 12

S-21E 19 MALMO

$\triangle G R I$ ISR BERNART L MR

P $\mathrm{P}$ SQ 2 SLAY

F-FII ${ }^{2}$ GIF-YVETTE
AAIJESTAD PER ARNE DR

PFYSICS DEPT

ARMPE AZ 85281

U.S.A.

AEHASOV ALIK R DR

STEMAKHA ASTRO-

PFYSICAL OESERVATORY

373243 SHEMAKHA AZER

U.S.S.R.

AEELE M K CR

LATVIAN STATE UNIVER

SITY ASTRONOMICAL

OBSERVATORY

22609 Q RIGA

U.S.S.R.

ABHYANKAR KRISHNA D PROF

DEPT CF ASTRONOMY

OSMANIA UNIVERSITY

HYDERABAD 500 CO 7

INDIA

AROU-EL-ELLA MOHAMED S DR HEL WAN OBSERVATORY

HELWAN CAIRO
ARAB REP. OF EGYPT

APRAMI ALBERTO PROF

OSSERVATOR IO ASTRONO MICD DI TRIESTE

I TAL Y

ABT HELMUT A DR

KITT PEAK NATL OBS

BCX 26732

TUCSON AZ 85726

U.S.A.

ACTON LOREN W DR

LOCKHEEO PALO ALTO

RESEARCH LAB

3251 HANOVER ST

PALO ALTO CA 94304

$U \cdot S \cdot A$.

ADAMS DAVID J DR

ASTRONOMY DEPT

LEICESTER LEI 7RH

U.K.

AOEL SHARAF MOHAMED DR

CEPT OF ASTR ONOMY

CAIRO

ARAB REP. OF EGYPT

AFANASJEVA PRASKOVYA M DR PULKOVO OB SERVATORY 196140 LEN INGRAD

$U . S . S . R$.

ACUERO ESTELA L DR

OES ASTRONOMICO

5000 CORDOBA

AR GENTINA
AARSETH SVERRE J DR

INST OF ASTRONOMY

MADINGLEY RD

CAMBRIDGE CB3 OHA

U.K.

ABBOTT WILLIAM N DR MICHALACOPOULOU 42 ATHENS 612

GREECE

$\triangle B E L L$ GEORGE $O$ PROF

DEPT OF ASTRONOMY

UNIVERSITY OF

CALIFORNIA

LOS ANGELES CA 90024

U. S.A.

ABLES HAROLD D OR

US NAVAL OBSERVATCRY $P O$ ROX 1149

FLAGSTAFF AL B6OOJ

U.S.A.

ABRAHAM HENRY J $M$

7 CASTLEREAGH

CRE SCENT

MACOUARIE ACT 2614

AUSTRALIA

ABRAMOWICZ MAREK DR N COPERNICUS ASTRON

CENTER

BARTYCKA 18

OO-7IG WAR SAW

POLAND

ABU EL ATA NABIL UR

BUREAU D LONGITUDES

77 AVENUE DENFERTE

ROCHEREAU

F-75014 PARIS

ADAM MADGE G DR

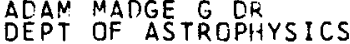

SOUTH PARKS RD

OXFORD OXI $3 R Q$

U.K.

ADAMS THOMAS F DR G-7 MS 329 LOS ALA-

$P$ OOX 1663

LOS ÁLAMOS NM 87545 U.S.A.

ADELMAN SAUL J DR

THE CITADEL

CHARLESTON SC 29409

U.S.A.

AGEKJAN TATEOS A.PROF

UNI VERSITY OF

LENINGRAD

DBSERVATORY

U. S.S.R.

AHLUWALIA HARJIT SINGH DR

DEPT PHYSICS ASTRON

UNI V OF NEW MEXICD

800 YALE BLVD N E

ALEUQUERQUE NM 87131

U.S.A. 
$\triangle L^{N} E S$ INAM IBRAHIM HELWAN CESERVATCFY HELWAI NEAR CAIRO $\triangle R A B K E P$. OF FGYPT

$\triangle$ IKAWA TOSHIKI CE ASTRCNCMICAL INST TCHCKU UNIVERSITY JEPA

AITKEN CAVIC K CR

DEPT PHYS AND ASTRO UNIV CCLLEGE LONDON GCWER STREET

LCNEON WCIE GBT

U.K.

AKAPANE KENJI A PROF TCFYC ASTRONOMICAL

CESERVATORY

CSAWA NITAKA

TCKYO 131

JAPAN

AKSENCV E P DR

TTE RUEFRG STATE AS-

117234 MOSCOW

U. S.S.R.

$A L-S \triangle D T I$ ABDUL ACIM CR

PHYSICS DEPT

SCIEVCE COLLEGE

BAGHLAO UNIVERSITY

$\mathrm{EACHCAC}$

HEP. OF IRAO

ALDERS HENRY PROF

VASSAR COLLEGE

CESERVATORY

PCUGHKEEPS IE NY 12601

U.S.A.

ALCRCVANDI SUELI MV DR

INSTITUTO ASTRONUMI

CC E GEOFISICO

CP $3: 627$

CRAZIL

$\triangle L F V E N$ HANNES PROF

CEPT CF PLASMA

PFYSICS

RCYAL INST OF TECHN

SWODEN 44 STOCKHOLM

ALKSNIS ANCREJS DR

RADICASTROPHYSICAL

CBSERVATORY

226524 RIGA LATVIA

U.S.S.F.

ALLEA CW PROF

NTS STRCMLD

WCDEN PC ACT 2606

AUSTRALIA

ALLEA RCNALD J DR KAPTEYN LABORATOR IUM

FCSTEUS BOO

9700 AV GRONINGEN

THE NETHERLANDS
AHNERT $P$ DR

ZENTRAL INST FUER

ASTROPHYSIK

STERNWARTE SONNEBERG

CLR-64 SONNEBERG

G.D.P.

A IKMAN GEORGE C L DR DOMIN IDN ASTROPH OBS $5071 W$ SAANICH ROAD

VICTORIA $B C \quad$ V8X $3 \times 3$

CANADA

AIZENMAN MORRIS L DR

DIV ASTRONOMICAL SC

NAT SC FOUNDATION

RM 615 1800 G ST NW

WASHIN

U.S.A.

AKABANE TOKUHIDE DR

HIDA OBS SER VATORY

506- 13 KAMITAKARA

GIFU

JAPAN

AKSNES KAARE DR.

NORWEGIAN DEFENCE

RESEARCH ESTABLI SHM

P O BOX 25

2007 K JELLER

NORWAY

ALAN IJA I F DR

AEASTUMANI A STROPHY-

SICAL OB SERVATORY

383762 ABASTUMANI

GEORGIA

ALBRECHT RUDOLF DR

INS F ASTRONOMIE

UNIVERSITAET WIEN

$A-I I 80$ WIEN

AUSTRIA

ALEXANDER JOH: B

ROYAL GREENWI'H OBS

HAIL SHAM

EAST SUSSEX BN27 IRP.

U.K.

AL I SSANDRAR \&S C PH D

LAB OF ASTI DPHYSICS

UNIV OF ATHENS

PANEP ISTIMIOPOLIS

ATHENS 621

GREECE

ALLADIN SALEH MOHAMED DR

DEPT OF ASTRONOMY

OSMANIA UNIVERSITY

HYOERABAO 500007

INDIA

ALLEN CHR I ST INE

INSTITUTO DE ASTROND

MIA UNAM

APDO POSTAL 70-264

MEXICO

$A L L E R$ HUGH D DR

DEPT OF ASTRONOMY

UN IVER SITY MICHIGAN

953 PHYS-ASTRO BLDG

ANN ARBOR MI 48109

U.S.A.
AIAD A Z DR

CAIRO UNIVERSITY

FACULTY OF SCIENCE

ASTRONOMY DEPT

GEZA ORMAN
ARAB REP. OF EGYPT

AIME C DR

DEPT DASTROPHYSI OUE

UNI VERSITE DE NICE

PARC VALRÓSE

F-O6034 NICE CEDEX
FRANCE

AIZU KO PROF

RIKKYO UNI VERSITY

NISHI-I KEBUKURO 3

TOSHIMAKU TOKYO 171

JAPAN

AKCAYLI MELEK M A DR

EGE UNI VERSITES I

GOK BILIMLERI ENST

BORNOVA IZMIR

TURKEY

AKYOL MUSTAFA U PROF

EGE UNI VERSITY

SCIENCE FACULTY

DEPT DF ASTRONOMY

BORNOVA IZMIR

TURKEY

ALBANO J DR

OBS ASTRONOMICO

ARGENTINA

ALDROVANDI RUBEN DR INSTITUTO DI FISICA TEORICA

RUA PAMPLONA 145

OI 405 SAO PAULO SP

BRAZI L

ALEXANDER JOSEPH $K$

CODE 693

GODDARD SPACE FLIGHT

CENTER

GREENBELT MO 20771

U.S.A.

ALKSNE ZENTA DR

RADIOAS TROPHYSI CAL

OBSERVATORY

TURGENEVA IO

226524 RIGA LATVIA

U.S.S.R.

ALLAMANDOLA LOUIS JOHN DR

STERREWACH

HUYGENS LAB

POSTBUS 9513

2300 RA LEIDEN

ALLEN DAYID A DR

ALGLN DAYSTR RALIAN

OBSERVATORY

PO BOX 296

EPPING NSW 2121

AUSTRALIA

ALLER LAWRENCE HUGH

ASTRONOOMY DEPT

UNIVERSITY DF CALIF

MATH-SCIENCES BLDG

LOS ANGELES CA 90024

U.S.A. 
ALLER MARGQ F CR

UATVERS ITY MICHIGAN

ANA ARECR MI 48109

U.S.A.

ALI AVISTA CARLOS A DR

PAS ASTRENOM ICO

1900 LA PLATA

ARGENTINA

ALTSCHULER MART IN D.PROF

CEPT CCN PUTER SC

STATE UNIVERSITY DF

NEW YCNK AT BUFFALO

ANHERST NY 14226

U.S.A.

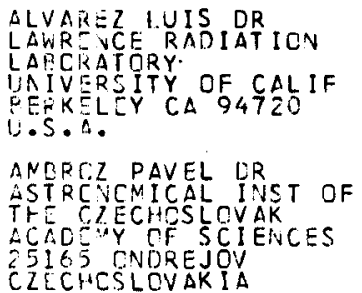

AMCEXSEN JOHANNES LEKTOR

BRCRFELDE OBS

CCPENHAGEN UNIV

LK -434 TCLLOSE

EENNAPK

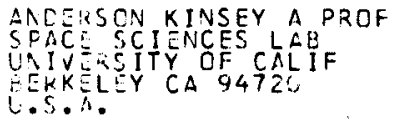

ANCREW KENNETH L PROF

DEPT OF PHYSICS

W LAFAYTTE IN 47907

U.S.A.

$\triangle N$ DEWW PETER J DR

RCYAL GREENWICH

CESEFVATCRY

HAILSHAN

CAST SUSSEX RN27 IR?

U.K.

AMTPILLAT HENRI L PROF

LAU TASTRONONIE

TECH LU LAITUEDOC

TANCE

ANGEL J RUGER P PROF

STEVARC OBSERVATCRY

UNIVE ZSITY OF

itk! 7 C NA

TUESC $A Z$ 85721

U. $\$ . A$.

AAOUITA CAA DR CHILE

CES ASTRCN NACICNAL

CASILLA 36-C

SAAT ASC

CAIL:
ALLOIN DANIELLE DR OBSERVATOIRE DE NICE F-O 6007 NICE CEDEX FRANCE

ALTENHCFF WILHELM J CR RACIOASTRONOMIE

AUF DEM HUEGEL 69

D- 5300 BONN

ALURKAR $S K$ DR

PFYSICAL RES LAB

NAVRANGPURA

AHMEDABAD 380009

INDIA

ALY M K PROF DIRECTOR

RABITATUL AALAM AL-

ISLAMIIC OBSERVATORY

MECCA ALMUKARRAMA

SAUDI ARABIA

ANAND $S P S D R$

APPL IEO RESEARCH AND

1996 GENERALS HWY

ANNAPOL IS MD 21401

U.S.A.

ANDER SON ERYAN OR

UN IV OF MANCHESTER

JODRELL BANK

MACCLESFIEED

CHESHIRE SKII 9DL

U.K.

ANDO HIRQYASU DR

TOKYO ASTRONOMICAL

OFSER VATORY

OSAWA 2-21-1 MITAKA

TOKYO

JAPAN

ANIDREWS DAVID A DR

ARMAGH OBSER VA TORY

ARMAGH BTEI $90 \mathrm{G}$

U.K.

ANOR I ENKO DMITRY A DR

VLADIMIRSKAYA 5153

FLAT 75

252 ? 3 KIEV

U.S.S.R.

ANDR ILLAT YVETTE DR

DE HAUTE-PRDVENCE

ST-MICHEL L OBS

F- 04300 FORCALQUIER

FRANCE

ANGELETTI LUCIO DR

OSSERVATURIO ASTRO-

NCNICC DI RCMA

I TAL $\mathrm{Y}$

ANILE ANGELO M

OSSERVATOR JO

ASTROFISICO

CITTA UNIVERSITARIA

I 95123 CATANIA

ITALY
ALMAR I VAN DR

SATELLITE GEODETIC

OB SERVATORY

POB 546

1373 BUDAPEST

HUNG ARY

ALTROCK RICHARD C DR AIR FORCE GEOPHYSICS

SACRAMENTO PEAK, OBS

SUNSPOT NM $8 B 349$

U.S.A.

ALVAREZ HECTOR DR

DEPTC DE ASTRONOMIIA

CASILLA 36-D

SANTI AGO

CHILE

AMBARTSUMIAN $Y$ A PROF DR B YURAKAN ASTROPHYSI

CAL OBSERVATORY

U.S.S.R.

ANDERS EDWARD PROF

UNI VERSITY OF

CHICAGO

5649 SELLIS VVE

CHICASO IL 60637

U. S.

ANDERSON CHRISTOPHER M OR WASHBISR OBSERVATORY

UNI VERSITY OF

WISCONSIN

MADISON WI 53706

U.S.A.

ANDREW BRYAN H DR

HERZEERG INST DF

ASTROPHYSICS

NAT RES COUNCIL

OTTAWA ONT KIA ORG

CANADA

ANDREWS DONALO H DOMINION ASTRO OBS NAT RES COUNC CANADA 5071 WEST SAANICH RD VICTORIA BC $\vee 8 X \quad 3 \times 3$ CANADA

ANORIESSE CORNE LIS D DR KAPTEYN STERREWACHT

MENS I NGHEWEG 20

RODEN DP

THE NETHERLANDS

ANDRLE P DR

A STRONONICAL INST

CZECH ACAD

12023 PRAHA 2

CZECHOSLOVAKIA

ANGIONE RONALD J DR

ASTRONOMY DEPT

UAN DIEGE

SAN DIEGO CA 92182

U.S.A.

ANSARI $S M$ RAZAULLAH DR PHYSICS DEPT

ALIGARH MUSLIM

UN I VERSITY

ALIGARH UP 202001

INOIA 

AATCNACCPOULCS CREG PROF LAIVERSITY OF PATRAS PATRAS

oDEECE

ANZER ULRICH DE UHC ASTPCPFYSIK

FCEHKINGER RING

GER RASY, F.R.

APPEIISLLER IMMO PROF

LANLESSTERNWARTE

K. EENIOSTUHL

$C-69$, HEI DELBEFC 1

GERNNNY, F.R.

ARCAVAI H DR

INST CF ASTRCNOMY

NAL 1 ILEY ROAD

CAM $⿴ 囗 十$ ICCE

l. K.

AROUL A NOEL MR

INST LF ASTRONONY

THE CBSERVATORIES

CALINOLEY RD

U.K.

ARIAS DE GREIF JURGE PROF

CESEOVATCRIO

NACIC:NAL

APARTACC 2584

PCGCTA

CELCN IA

ARNCULE MARCEL L DR

INST DAS LIB BRUXELLES

AVE F C RCOSEVELT 50

BELGIUM

ARCNS JONATHAN

UEPT OF ASTRONOMY

UNIVERSITY OF CAL IF

RERKELEY CA $9472 \mathrm{C}$

U.S.A.

ARTHUR DAVID W. G

US GFOLOGICAL SURVEY

FLAGSTAFF ARIZONA

U.S.A.

$\triangle S C H E N H A C H$ BFRNC PH C

HEIT RWANGERSTR 54

D-dO? NUENCHEN 7 :

GERNAVY, F.R.

ASLANOV I A DR

CHIENAKHA ASTRRPHYSI-

CAL CBSERVATORY

373243 AZEREAICZAN

U.S.S.K.

ASTEPI ADIS GEORCIOS DR IESTRENOMY G

UAIV THESSALONIKI

THESSALCNIK

GREECE
ANTONOV V A DR.

LENINGRAD STATE UNI-

VEKSITY ASTRONOMICAL

OBSERVATORY

199178 LEN INGRAD

U.S.S.R.

AOK I SHINKO PROF

TOK YO ASTRONOMICAL

OESER VATOPY

MITAKA

TOKYO 181

JAPAN

ARA I KENZO DR

DEPT OF PHYSICS

KUMAMOTO UNI VERSITY

JPAN

ARDEBERG ARNE L DR

LUND OBSER VA

$S-222 \quad 24$ LUND

SWEDEN

ARGYLE P E DR

DOMINION RADIO

ASTROPHYSICAL OBS

PCX 248 ONT BC V2A $6 \mathrm{~K} 3$

CANADA

ARKHIPOVA V P DR

STERNEERG STATE AS-

TRONOMICAL INSTITUTE

117234 MOSCOW

U.S.S.R.

ARNQUIST WARREN N DR 8127 DELGANY AVE PLAYA DEL REY CA 90291

U.S.A.

ARP HALTON DR

HALE CESER VATORIES

813 SANTA BARBARA ST PASADENA CA 91101

U.S.A.

ARTRU M C DR

OBS DE MEUDON

FRANCE

ASHBROOK JOSEPH DR

16 SUMMER ST

WESTON MA C2193

U.S.A.

ASSEO ESTELLE DR

OQSERVATOIRE DE

MEUDON DAF

$F-92190$ MEUDON

FRANCE

ATANASIJEVIC IVAN OR.

FACULTY OF SCIENCE

65OO GL NI JMEGEN
ANTONUCCI ESTER OR

UNIVERSITA DI TORINO

C D AZEGLIO 46

ITALY'

APPARAO K M VOR

TATA INST FUND RES

HOMI BHABH ROAD

BDMBAY 400005

INOIA

ARAKELIAN MARAT A DR BYURAKAN ASTROPHYSICAL OBSERVATORY $37 \$ 433$ EYURAKAN ARMENIA

U.S.S.R.

AREND $S$ DR

AVENUE DE SATURNE 11 B- 118 RRUXELLES

BELGIUM

ARGYRAKOS JEAN PROF DR

UNIY TECHNI QUE

NATI ONALE D ATHENES

193 PATISSIOV ST

ATHENS 816

GREECE

ARNETT W DAVID PROF

ENRICO FERMI INST

933 E 56TH STREET

CHICAG

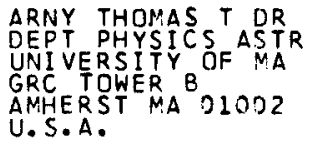

ARPIGNY CLAUDE PROF

INST D ASTRCPHYSIQUE UNIVERSITE DE LIEGE BELGIUM

ASAAD ADLY S PROF

DBSERVATORY OF

HELWAN

HELWAN

ARAB REP. OF EGYPT

A SLAN ZEKI DR

UNIV OF ANKARA

FEN FAKULTESI

ANKARA

TURKEY

ASSOUSA GEORGE ELIAS DR

DEPT TERR MAGNETISM

DEPT TERR MAGNET ISM

5241 EROAD BR RD N W WASHINGTON DC 20015 U.S.A.

ATHANASSOULA E D DR OBSERVATOIRE

DE BESANCON

4 IB AVE OBSERVATOIRE

FRANCE 
$\triangle T H A Y R$ GRANT DR

HIGH ALTITUDE

CFSEXVATOKY

PCLLLER CO 80302

U.S.A.

AUECULE JEAN CR

INST OASTROPHYSIQUE

IS B IS BLVD ARAG

$F-75,14$ PARIS

FRANCE

\section{AUNAN JASLN R PROF \\ LEPT OF GEOPHYSICS \\ AINE ASTRCNDMY \\ UAIVERSITY OF PC \\ VANCCUVER BC VGT IWS \\ CANACIA \\ AVERY LCRNE W CR \\ HERZHERG INST DF \\ ASTRGIPHYSICS \\ NAT RES CDUNC CANADA \\ CTTAWA CNT KIA ORG \\ CANADA \\ AVRETT EUGENE H CR \\ CE YTEP FOR \\ ASTRCPHYSICS \\ 60 GARCEN ST \\ CANHRIEGE MA 02133 \\ U.S.A. \\ A7ZCPAROI M MR \\ CES DE TOULOUSE \\ F-315L; TOULOUSE \\ FRANCE}

PAPACZHANIANC MICHAIL CR

ASTRCINOMICAL OBS

LENINGRAD UNIVERSITY

LEAINGRAD

U.S.S.R.

PACCHUS PIFRRE PROF

1 INPASSE DE

L-CESERVATOIRE

$F-59000$ LILLE

FRANCE

HAEHR ULRICH DR

QUIACKESTRASSE 44A

D-69:O HEIDEL BERG

GERMANY, F.R.

PAGHOS BALEGH B DF

HELWAN CBSERVATORY

HELWAN CAIRO

ARAQ REP. OF EGYPT

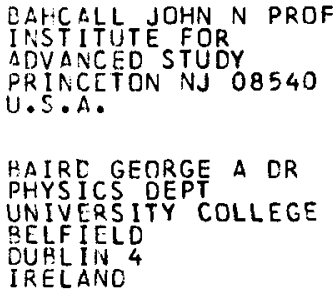

ATKINSON ROBERT D E DR

SWAIN HALL WEST 319

INDIANA UNIVERSITY

BLOOMINGTON IN 47401

U.S.A.

AUER LAWRENCE H DR

HIGH AL TI TUDE

OBSERVATORY

$P$ O BOX 1470

BOULDER CO BO302

U.S.A.

AUNER GERHARD OR

INST FUER ASTRONOMIE

UN IVERSITAETSSTR 4

A- 6020 INNSBRUCK

AUSTR IA

AVIGNON YVETTE DR

OBSERVATOIRE

DE MEUDON

F-O2190 MEUDON

FRANCE

AXFORO W IAN PROF

MPI FUER AERONOMIE

POSTFACH $2 \mathrm{C}$

$D-3411$ KA TLENBURG-

LINDAU 3

GERMANY, F.R.

BAARS JAAP W M DR

MPI FUER

RACIOASTRONOMIE

AUF DEM HUEGEL 69

D-5300 BONN

GERMANY, F.R.

BABADZHANCV $P$ B DR

ASTROPHYSICAL INSTI -

TUTE OF TADJIKIAN

ACADEMY OF SCIENCES
734670 DUSHANBE

U.S.S.R.

BACKER DONALD CH OR

GOI CAMPBELL HALL

UN IV OF CALIFORNIA

BERKELEY CA 94720

U.S.A.

BAERENTZEN JORN

ELMEHOJVEJ 66

DK-B270 HOJAJERG

DENMARK

BAGILDINSKIJ BRONISLAV

KULKOVO OBSERVATORY

196140 LENINGRAD

U.S.S.R.

BAHNER KLAUS DR

LANDES STERNWAR TE

KOENIGSTUHL

D-6900 HE IDELBERG 1

GERMANY, $F$.R.

BAIRD KENNETH M DR

DIVISION OF PHYSICS

NAT RES COUNC CANADA

MONTREAL ROAD

OTTAWA DNT KIA ORG

CANADA
AUBIER MONIQUE G DR

DBSERVATOIRE DE

MEUDON
$F=92$ I9O MEUDON

FRANCE

AULUCK F C PROF

DEPT OF PHYSICS AND

ASTROPHYSICS

UNI VERSITY QF DELHI DELHI 110007

INDIA

AURIEMMA GIULIO DR

CNR ASTROF IS ICO

SPAZI ALE C P 67

I -00044 FRASCATI

ROMA

ITALY

AVNI YORAM DR

WE I ZMANN INST I TUTE REHOVOT

I SRAEL

AYOIN CEMAL ASSOC PROF

AU FEN FAKULTESI

ASTRONOMI BOLUMU

BESEVIER ANKARA

TURKEY

BAART EDWARD E PROF

DEPT OF PHYSICS

RHODES UNIVERSITY

P O BOX 94

GRAHAMSTOWN 6140

SOUTH AFRICA

BABCOCK HORRACE W DR

HALE OBSERVATORIES

813 SANTA BARBARA
PASADENA CA 91101

U.S.A.

BAECK NICOLE A L DR 195
$B-9218$
B

BELGIUM

BAGGALEY WILLIAM JACK DR

PHYSICS DEPT

UNIV OF CANTERBURY

CHRI STCHURCH

NEW ZEALAND

BAGLIN ANNIE DR OBSERVATOIRE DE NICE F-060O7 NICE CEDEX FRANCE

BAHNG JOHN D R PROF DEARBORN OBSERVATORY NORTHWESTERN

UNI VERSITY

EVANSTON IL 60201

U. S.A.

BAIZE PAUL DR

6 RUE DAUB I GNY

F-75017 PARIS

FRANCE 
FAJAJA E DR

INSTITUTO ARGENTINO

OE RACIOASTRONOMIA

CAS ILLA NO 5 $V I L L A$ ELISA PR PS AS $\triangle$ PCENTINA

EAKCS GUSTAV A PROF

IEPT OF PHYS ICS

UNIVERSITY WATERLCO

CANALA

BALDIPELLI LUIGI DR I -40141 BOLOGNA

IT $\triangle L Y$

BALC II RALPH B

3110 NANHATTAN LANE

GRAND RAPIDS MI49506

U.S. $\Lambda$.

ALL JCHN A DR

CBSERVATORY

HARVARL MA O1451

U.S. A.

EALN IIS GEORGES C TER CTR NAT D'ETUCES SPA GRLUP RECH GEOD SPAT $1 \&$ AV EQDUARC QELIN $F-314 \%$ TOULOUSE

FKANCE

GASCYOPADHYAY A CR

INCIA NETED DEPT

INCI AN ASTRON EPH UN

P546 OK N NW ALIPORF

CALCLITA 700053

INCIA

BANCS GECKRE S PROF

I CANAina

ASTQCIOMY DEPT

IOAMAINA

GRECE

$\triangle D R \triangle T T A G B D R$

ESEFTVATCRIO ASTRO-

NUNICO OI ROMA

$1-0,1390$ RCMA

IAREIZK MADELEINE AST ADJ

CPSE RVATCIRE

C. NAQS ILLE

F PLACE LEVERRIER

FRANCE

HARCLA SZABCLCS CR

KCARCLY OESERVATOJPY

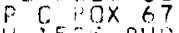

H- 523 BLUAPEST

HLICARY,

IARKER EOWIN S CR

MCIIC ALE OBSERVATORY

TCKT UNVIS TX 79734

U.S. .
BAKER JAMES GILBERT DR WINCHESTER MA 01890

U.S.A.

BALAZS BELA A DR

CEPT OF ASTRONOMY

EOTVOS UN I VER SITY

KUN BELA TER 2

HONGARY
HUNG

BALDWIN JACK A DR

INST OF ASTRONOMY

MADINGLEY ROAD

CAMER IDGE CB 3 OHA

U.K.

BALICK BRUCE PROF

ASTRONOMY DEPT FM-20

UNIV OF WASHINGTON

SEATTLE WA 98195

U.S.A.

BALLARIO M C PROF

USSERVATORIO ASTRONO

MICO DI ARCETRI

VIA S LEONARDO

ITALY 50 FIRENZE

BALQNA LUIS ANTERO DR

S A ASTRON OBS

QESERVATORY 7935

CAPE

SOUTH AFRICA

BANIN $Y G$ DR

P IE I I 4 M

6t4 697 IRKUTSK 33

U.S.S.R.

RAPPU M K V DR

DIRECTOR

INDIAN INSIITUTE OF

ASTROPHYSICS

BANGALORE 560034

INOIA

BARBANIS BASIL PROF

OEPT OF ASTRONOMY

UNIVERSITY OF PATRAS

PATRAS

BARBIERI CESARE PROF

INST DI ASTRONOMIA

UNIVERSI TA DI PADOVA

IT 35100 PADOVA

BARDEEN JAMES M PROF

PIYSICS DEPT FM- 15

UNIVERSITY OF

WASHINGTON

SEATTLE WA 38195

U.S.A.

BARKHATOVA KLAUDIA PRUF

STATE UM IVER SITY

LENIN ST

620083 SVERDLOVSK

U.S.S.R.
BAKER NORMAN H PROF

ASTRONOMY DEPT

COLUMBIA UNI VERSITY

PUPIN HALL 538 W 120 NEW YORK N Y IOOL27 U.S.A.

BALAZS LAJOS G DR KONKOLY OBSERVATORY HOI 1525 BUDAPEST HUNGARY

BALDWIN JOHN E DR CAVENDI SH LABORATORY MADINGLEY RO CAMBRIDGE CB3 DHE U.K.

BALKLAVS A E DR RADI OAS IROPHYS I CAL OBSERVATORY LATVIAN ACADEMY OF SCIENCES 226026 RIGA U.S.S.R.

BALLI EOIBE PROF

UNI VERS ITE

ISTANBUL

I STANBUL
TURKEY

BANDERMANN L WR

ROYAL OBSERVATORY ELACKFORD HIL ${ }_{\text {EI }} L_{3} \mathrm{H}$ U. K.

BANOS COSMAS J DR DBSER VATOIRE NATIONAL D A THENES A THENS 306

GREECE

BARANNE A DR

OBSERVATOIRE 2 PLACE LE VERRIER F-I3OJ4 MARSEILLE FRANCE

BARBARO G OR

OSSERVATORIO ASTRON VICOLO DELL PADÕVA

I TALY

BARBON ROBERTO PROF OSSERVATORID ASTROFISICD I TALY

BARKAT ZALMAN PROF THEORETICAL PHYSICS HEBREW UNIVERSITY JERUSALEM

ISRAEL

BARLAI KATALIN OR KOIIKOLY DBSERVATCRY BOX 67

H- 1525 BUDAPEST 
BARLTTI RAFFAELE ENG CSSERVATCRIO ARCETRI $\angle A R G C$ FERMI 5 I $=50125$ FIRENZE

EARNARD HANNES A J DR PHYYSICS DEPT

UNIVERS ITY OF $E$ C

2,75 WESBROOK MALL VANCCUVER VGT IWG CANALA

I3APNCTHY JENO DR PROF Y3 3 LINCOLN ST
EVANSTCN IL 60201 i...$A$.

LAFRETT ALAN H PROF NCON $2 E-331$ C. TCCHNCLOGY U.S.A.

H.ARRY UCN C OR

UAIVERSITY OF

SLUTHERN CAL IFORNIA

LCS ANGELES CA 90007

U.S.A.

DARTHOLDI PAUL CR

COSERVATCIRE

UE GENEVE

CH-1 29: SAUVERNY GE

SWITIEKLAND

EASCHEK POOO PROF

INST F THEORET ISCHE

ASTRCPHYS IK

IN NEUENHEIMER F 294

$[-6,3]$ ] HE I DELEER

GERVANY, T.R.

EASU PAIOYAYATH REACER

AFPLI D NATHEMATICS

DEPARTNENT CU

92 P P C ROADO

INOIA

PATES CAVID R PRCF

CEPT QF APPLIED NATH

CUEENS UNIVERSITY

RFLF IST BT7 INN

U. $k$.

ELTH SECFFREY T DR

DEPT OF ASTROPHYS ICS

UNIVERSITY OF OXFORD

CXFCRU CXI $3 R Q$

1,. K.

BATTEN ALAN H DR

QCNINICN ASTROPFYS

CESFRVATORY

5.71 W SANICH RL

VICTCRIA HC VBX $3 \times 3$

CANACA

FAUEQ CARL A QR

PENNA STATE UNIV

UAIV PK PA 16802

U. . A.
BARLIER FRANCOIS E DR CER GA

AV COPERNIC

F-06130 GRÁSSE

FRANCE

BARNES AARON DR

NASA AMES RES CTR

MODE $245-3$ FIT CA 94035

U.S.A.

BAROCAS VINICIO DR

JEREM IAH HORROCKS

AND WILFRED HALL OBS

MOQR PARK

PRESTON PRI GAD

U.K.

BARROW COLIN H DR

CEPT OF PHYSICS

WEST INDIES

KINGSTON W I 7

JAMA ICA

BARTAYA R A DR

ABASTUMAN I A STRDPHY-

SICAL OBSERVATORY

383762 ABASTUMANI

GEORGIA

U.S.S.R.

BARTOL INI CORRADO

OSSERVATOR IO

ASTRONOMICO

$\checkmark$ IA ZAMBONI 33

I 40126 BOLOGNA

BASH FRANK $N$ PROF

ASTRONOMY DEPT

UN IVERSI TY OF TEXAS

AUSTIN TX 78712

U.S.A.

BASU DIPAK DR

DEPT OF PHYSICS

WEST INDIES

ST AUGUSTINE

TRINIDAD AND TUBAGO

BATES RICHARD HEATON T DR

ELECTR ICAL ENG DEPT

UN IV OF CAN TEREURY

CHR I STCHURCH

NEW ZEALAND

BATRAKOV J $M$ DR

INSTITUTE OF THEORE-

ICAL A SIRONOMY

U.S.S.R

BATTISTINI PIERLUIGI DR

OS SER VATORIO

ASTRONOMICO

VIA ZAMRON I 33

ITALY

BAUM WILLIAM A DR

LOWELL OB SERVATQRY

$P O B O X 1269$

FLAGSTAFF AZ 86002

$U: S . A$.
BARLOW MICHAEL J DR ANGLO AUSTRALIAN OBS $P$ O BOX 296

EPPING NSW 2121

AUSTRALIA

BARNES II I THOMAS G DR

DEPT DF ASTRONOMY

UNI VERSITY OF TEXAS

AUSTIN TX 78712

U.S.A.

BARRETO LUIZ MUNIZ PROF OBSERVATOR I O

NACI ONAL

RUA GEN BRUCE 586

2 DOOO RIO DE JANEIRO BRAZIL

BARROW RICHARD F DR

PHYSICAL CHEMISTRY

LABORATORY

SOUTH PARKS RD

OXFORD OXI $3 Q Z$

U.K.

BARTH CHARLES A PRDF

LASP UNIVERSITYY

OF COLORADO

BOULDER CD 80309

U.S.A.

BASART JOHN P

NATR RADIOASTRON OBS

$P$ O BOX O

SOCORRO NM 87801

U.S.A.

BASTIN J A PROF

PHYSICS DEPT

QUEEN MARY COLLEGE

MILE END ROAD

U.K.

BATES BRIAN DR

DEPT OF PURE AND

APPLIED PHYSICS

QUEENS UNI VERS ITY

BELFAST BT7 INN

U.K.

BATESON FRANK $M$ OBE $P$ O BOX 3093

GREERTON TAURANGA

NEW ZEALAND

BATTANER EDUARDQ DR

INSTITUTO DE ASTROF I

SICA DE ANDALUCI A

APDO 2144

GRANADA

SPAIN

BAUDRY ALAIN DR

OB SERVATOIRE

DE BORDEAUX

F-33270 FLOIRAC

FRANCE

BAUSTIAN WW MR

KITT PEAK NAT OBS

$950 \mathrm{~N}$ CHERRY AVE

P O BDX 26732

TUCSON $A Z 85726$

U.S.A. 


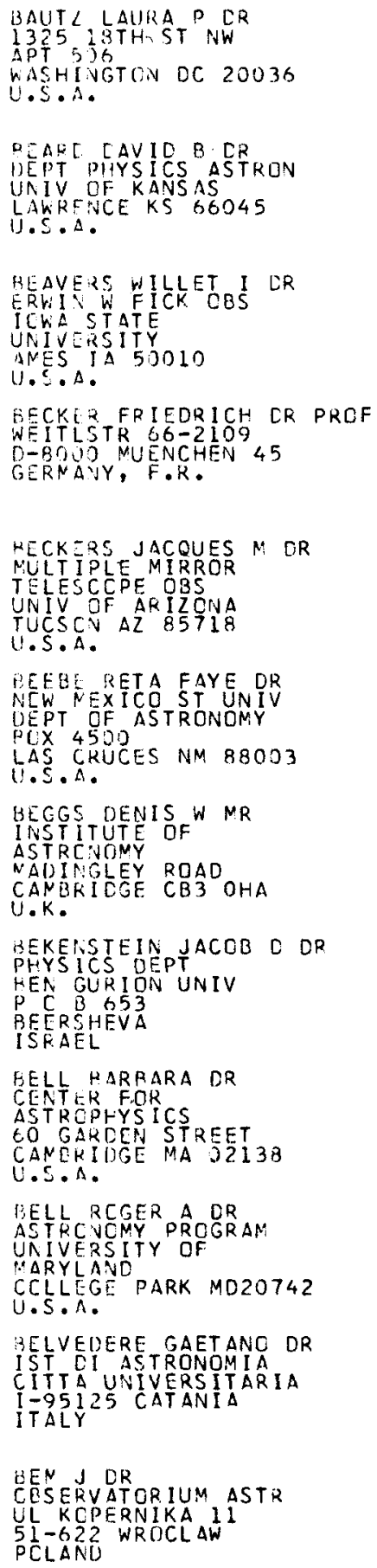

BAYM GORDON ALAN DR DEPT OF PHYSICS

URBANA IL $61 B O 1$

U.S.A.

BEARDSLEY WALLACE R DR

DEPT PHYS AND ASTRON

UNIV OF PITTSBURGH

ALLEGHENY OBS B 7658

PITTSBURGH PA 15214

U.S.A.

BEC-BOR SENBERGER ANN I CKDR

BUREAU LONGITUDES

ROCHEREAU

$F-75014$ PARIS

FRANCE

BECKER ROBERT A DR

$P$ BOX 4609

CARMEL CA 93921

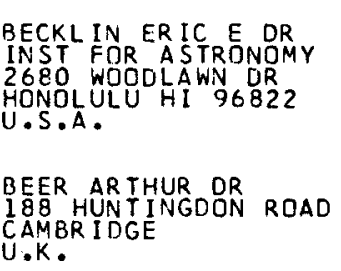

BEHR ALFRED PROF HAMBURGER SIERNWARTE GOJENBERGSWEG 112 DE 2050 HAMBUR

BEL NICOLE J DR OBSERVATOIRE DE MEUDON F-92190 MEUDON FRANCE

BELL KENNETH LLOYD DR DEPT OF APPLIEO MATH AND THEORET PHYSICS QUEEN 'S UN I VER SI TY

BELFAST BT 7 INN U.K.

BELOTSERKOVSKI J

DAVID J DR GOSSTANDART USSR LEN INSKY PROSPECT 9 117049 MOSCDW U.S.S.R.

BELY OLEG DR OBSERVATOIRE DE NICE B P 252
F-O6007 NICE CEDEX
FRANCE BENAVENTE JOSE DE MAR INA SAN FERNANDO CADIZ SPAIN
BEALE JOHN S DR ROYAL GREENWICH OBSERVATORY

HAI LSHAM

EAST SUSSEX BN27 IRP U. K.

BEAUDET GILLES DR DEPT DE PHYSIQUE CP 6128 MONTREAL PO H3C $3 \mathrm{~J} 7$ CANADA

BECK H G

VEB CARL ZEISS

FORSCHUNGSZENTRUM CARL ZEISS STR 1 DDR 69 JENA

G.D.R.

BECKER WILHELM PROF ASTRON INST UNIVERSITAET BASEL VENUSSTR 7 CH- 4102 BINNINGEN SWITZERLAND

BECKMAN JOHN E DR DEPT OF PHYSICS

MILE END ROAD

LONDON E1 4 NS

U.K.

BEER REINHARD DR

JET PROPULSION LAB

CALIF INST DF TECH

$480 O$ OAK GROVE DR

PASAOENA CA 9 IIO3

U.S.A.

BEINTEMA DOUWE A DR UNIV OF GRONINGEN P O $80 X 800$ GRONINGEN

THE NETHERLANDS

BELKOVICH O I DR ENGELHAROT ASTRONOMI CAL OBSERVATORY 422526 KAZAN U.S.S.R.

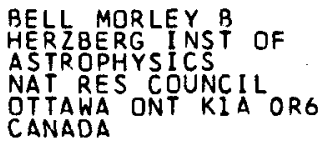

BELTON MICHAEL JS DR SOLLAR SYSTEM PROG $950 \mathrm{~N}$ CHERRY AVE TUC SON AZ 85726 U.S.A.

RELY-DUBAU FRANCOISE OBSERVATOIRE DE NICE B-P 252
F-O6OO 7 NICE CEDEX
FRANCE

BENDER PETER L DR

JILA

UNIVERSITY OF

COLORADO

BOULDER CO 80309

U.S.A. 
13ENEOICT G F DR

UAIV CF TEXAS

AUSTIN TX 78712

U.S.A.

BENFCRD GREGORY DR

PHYSICS DEPT

UAIV OF CALIFORNIA

IKVINE CA 92717

U.S.A.

\section{BENVENUTI P DR \\ OSSERVATORIO ASTRO- F IS I CO \\ I $-361 \mathrm{I} 2$ ASIAGO}

WEKS RICHARD A DR

DEPT PHYS AND ASTRON

UNIV OF ROCHESTER

419 SPACE SC 14627

R.C.HEST.

PEKGER CHRISTIANE DR

CIRCA

AVENUE COPERNIC

F-613: GRASSE

FRANCE

PERCIRCN JACOUFLINE A DR ESC C-C CERN

CH-1ZII GENEVE 23

SUITIERLAND

PERNAI ROHERT HIRAM OR IT

KN $36-227$

77 NSSACHUSETTS AVF CAMHFIDOE MA OZ139

U.S.A.

LERT AUL CHARLES DR

CHSEPVATCIRE

TES NGLCNEUDON

FRANCE

IEKTIAUS FLOR C PROF

WAVERS SPAAN 220

D-303? HEVERLEE

PELOIUN

BERTCUT CLAUDE PH D

LANLISSTERNWARTE

KICENIOSTUUIL

C-GG HEIDELAERG 1

CERMAYY, F.R.

EELERMANN KLAUS P PROF

PH BEZLIN ABT 4

UALTSERSTR 74-100

T-IOJ PERLIN

BHATIA V B DR

DEPT OF PHYSICS ANC

OELHI UNIVERS ITY

CELH - 110007

INCIS
BENEST D DR

B P 252

F-06007 NICE CEDEX

FRANCE

BENNETT JOHN CAISTER DR

90 MALAN STREET

R IVIERA

PRETOR IA 0084

SOUTH AFR ICA

BENZ ARNOLD DR

RAOIO ASTRONOMY

GROUP ETHZ

HOCHSTR 58

CH-8O 44 ZUER ICH

SWITZERLAND

BERGE GLENN L DR

OWENS VALLEY RADIO

OBSERVATORY

CAL TECH 10224

PASADENA CA 91125

U.S.A.

BERGER JACQUES G DR

OBSER VATOIRE

DE PAR IS

F-75014 PARIS

BERGSTRALH JAY T DR

MPL $1838-365$

4 BOOO OAK GROVE DRIVE

PASADENA CA 91103

U.S.A.

RERNACCA $P$ DR

QSSER VATOR IO ASTRO-

FISICO

ASIAGO VICENZA

ITALY

BERTAUX J LDR

SER VICE D AERONOMIE

$P$ NO
F- $91370^{3}$ VERRIERES-

LE-BUI SSON

BERTIN GIUSEPPE DR

SCUOLA NORMALE SUP PIAZZA DEI CAVALIERI IT 56100 P ISA

BFSSELL MICHAEL S DR MT STROMLO

OBSERVATORY

WODEN PO ACT 2606

AUSTRALIA

BEYER MAX DR

JUSTUS-BR INCKMANN-

STRASSE 101

D-2O50 HAMBURG 80

GERMANY, F.R.

BHATNAGAR ARVIND DR

VEDHSHALA UDAIPUR

SOLAR OBS

II VIDYA MARG

UOA IPUR RAJAS 313001

INOIA
BENEVIDES SOARES $P$ DR

INSTITUTO ASTRONOMI -

CO E GEOF ISICO

CAIXA POSTAL $30 \quad 627$

BRAZIL

BENSAMMAR S DR

OBS DE MEUDON

F-92I9O MEUDON

BERENDZEN RICHARD DR

PROVOS

THE AMERICAN

UNIVERSITY

WASHINGTON DC 20016

U.S.A.

BERGEAT JACQUES G DR OBSERVATOIRE DE LYON F-69230 SAINT-GENIS-

LAVAL

FRANCE

BERGER XAVIER DR

CERGA

AVENUE COPERNIC

F-6130 GRASSE

FRANCE

BERKHUI JSEN ELLY M DR

MPI FUER

RADI OASTRONOMIE

AUF DEM HUEGEL 69

D-5300 BONN

GERMANY, F.R.

BERNAT ANDREW PLOUS DR KITT PEAK NAT OBS 950 NORTH CHERRY AVE $P$ O BOX 26732

TUCSON AZ 85726

U.S.A.

BERTHOMIEU GABRIELLE DR OESERVATOIRE DE NICE BP 252

F-06007 NICE CEDEX

FRANCE

BERTOLA FRANCESCO PROF

OSSERVATORIO

ITALY 35100 PADOVA

BETTIS DALE G PROF

UNIVERSITY OF TEXAS

AT AUSTIN

AUSTIN TX 78712

U.S.A.

BHATIA PREM K DR

DEPT OF MATHEMATICS

UNI VERSITY OF

JODHPUR

JODHPUR - 342001

I ND I A

BHATNAGAR $K$ B DR

ZAKIR HUSSAIN COLL

UNIV OF DELHI

$\triangle$ JMERI GATE

NEW DELHI IIO 006

INDIA 
FHATTACHARYYA J C PROF INC I AN INST ITUTE OF ASTREPHYS ICS

CANGALCRE 560034

INC I $\triangle$

EICARSCV RAVILISH CR

ASTRCPFYSICAL INST

TALJIK SSR

734675 DUSHANBE

U.S.S.R.

EIEGIVVG JOHN HAROLD DR

RADIC ASTRONOMY LAB

CANPUELL HALL

UAIV OF CALIFORN IA

PERKELEY CA 94720

U.S.A.

PIERNANN LUDWIG PROF DR

NPI F ASTKOPHYSIK

FCFHR INGER RING 6

D-EOOOD MUENCHEN 40

GERNANY, F.R.

BI JAIUI ALBERT DR

COSERVATOIRE DE NICE

8 P 252

F-O6JO7 NICE CECEX

FRANCE

HINCHAN RICHARD G DR RCY $\triangle L$ GREENW ICH

CESERVATORY

HAILSHAM

EAST SUSSEX BN27 IRP

U.K.

BIPAUD FRANCOIS DR

CBSEKVATOIRE

DE $N \subseteq U C C N$

$F-92190$ MEUDON

FRANCE

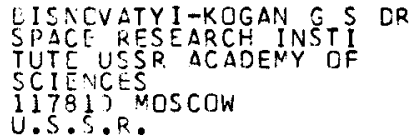

BLACK JOHN HARRY CR HARVARE COLLEGE OBS CTR TER AST RO

CANPKIOGE MA 02138

U..S.A.

ELAHA MILAN DR

CEPT OF PHYSICS AND

ASTRC UOMY

UAIV RSITY MARYLAND,

U.S.A.

EAACO CARLO DR

CSTERVATORIO

ASTRCF ISICD

CITTA UNIVERSITARIA

$1-95125$ CATANIA

ITALY

ILASIUS KARL RICHARD DR PLANET ARY SC INST

$283 \bar{S}$ LAKE

SUITE 218

PASATENA CA 91101

U.S.A.
8HONSLE RAJARAM $V$ PROF PHYSICAL RESEARCH

LABORATORY

NAVRANGPURA

AHMEDABAD 380009

IND IA

BICAK JIR I DR

DEPT OF MATH PHYSICS

CHARLES UNIVER SITY

POVLTAVSKA 8

180 OO PRAHA 8

CZECHOSLOVAKIA

BIEL ICK I MACIEJ DR

A STRONOMICAL

OBSER VATORY

AL UJAZOOWSKIE 4

OO-478 WAR SZAWA

POLAND

BIERMANN PETER L DR

MPI FUR RADIOASTRON

AUF OEM HUEGEL 69

D-530O BONN

BILLAUD G J

CERGA

ÁVE COPERN IC

F-6130 GRASSE

FRANCE

B INNENDIJK L PROF DR

FLOWER AND COOK

OBSERVATORY

UNIV OF PENNSYLVANIA

PHILADELPHIA PAI9104

U.S.A.

BIRKLE KURT PH

MP I FUER ASTRONOMIE

KOEN IGSTUHI

D-6900 HEIDELBERG 1

GERMANY, F.R.

BISWAS SUKUMAR DR

SOLAR PHYS GROUP

TATA INST FUND RES

HOM I BHABHA ROAD

BOMBAY 400005

IND IA

BLACKWELL DONALD E PROF

DEPT OF ASTROPHYSICS

SOUTH PARKS ROAD

OXFORD OXI $3 R Q$

U.K.

BLAIR GUY NORMAN DR

ASTRONOMY PROGRAM

DEPT EARTH SPACE SCS

SUNY

STONY BROOK NY 11794

U.S.A.

BLANCO VICTOR M DR

CERRO TOLOLO

INTERAMER ICAN DBS

CAS ILLA $E 3$ D

LA SERENA

CHILE

BLECHA ANDRE BORIS G OR 16 RUE ET-DUMONT

SH- 1204 GENE
B I ANCHINI ANTONIO DR OSSERVATORIO ASTROFISICO I 36012 ASIAGO

BIDELMAN WILLIAM P PRGF WARNER AND SWASEY

OB SERVATORY

1975 TAYLOR RD

E CLEVELAND OH 44112

U.S.A.

BIEMONT EMILE DR

RUE A LECLEROS 39

B-4652

BELGIUM

BIGAYJH DR

10 AVE DU GENERAL

DELESTRAINT

F-DIOOO BOURG-EN-

FRANCE

BILLINGS DONALD E PROF

UNI VERSITY COLORADO

DEPT OF ASTRO-

GEOPHYSICS

BOULDER CO 80309

U.S.A.

BINNEY JAMES J DR

I KEBLE ROAD

OXFORD OXI 3NP

U. $k$.

BISIACCHI GI ANFRANCO DR

INST DE ASTRONOMIA

UNAM

APDO POSTAL 70-264

MEXICO D F

MEXICO

BLAAUW A PROF DR

STERREWACHT

POSTBUS 9513

2300 RA LEIDEN

BLADES JOHN CH DR

ANGLO AUSTRALIAN OBS

$P$ BOX 296

EPPING NSW 2121

ÁUSTRALIÁ

BLAMONT JACQUES E PROF

SERVICE D AERONOMIE

DU CNRS B P 3

F-91370 VERRIERES

LE BUI SSON

BLANDFORD R D CR

INST OF ASTRONDM

MADINGLEY ROAD
CAMBRIDGE CB3 OHA

U.K.

BLEEKER JOHAN A M DR IR HUYGENS LABORATORY

WASSENAARSEWEG 78

2300 RA LEIDEN

THE NETHERLANOS 
ALESS KOPERT C PROF

LAIVERSITY WISCONSIN

475 CHARTER ST

NALISCN WI 53706

U.S.A.

SLUCNAN SIDNEY A PROF

DEPT OF PHYS ICS

UA AVERSITY OF

PENNEYLVANIA

PHILADELPHIA PAI9104

U.S. A.

BCERCV M S DR

ASTRENOMICAL COUNCIL

USSR ACAOEMY SCI

1OOOI7 MOSCOW

U.S.S.R.

POCENHEIMER PETER PROF

LICK OBSERVATORY

UNIVERSITY OF CAL IF

U.S.A.

DCEHME A OR

HEINRICH HERTZ INST

TELEGRAFENBERG

COR I5 POTSDAM

G.C.R.

ECERNGEN FRE IMUT CR PH ZENTR INST ASTR.CPHYS

$K$ SCHWH ARZSCHILD DAS

CLR-69G1 TAUTENEURG

$G \cdot C \cdot Q$.

PCEGESS $\triangle L B E R T$ CR

CCLE 673

GCCCARD SPACE

FLIGHT CENTER

GREENHELT MO 20771

U.S.A.

SCHLIN J DAVID CR

CCCE ST NASA HEADQUARTERS

WASHINGTON DC 20546

U.S.A.

QCISCHCT ANDRE DR

NEUCCN

F-9219O MEUDON

TRANCE

BCLCAL C DR

UAIV OPSERVATORY

UNIVERSITE

ISTANBUL

TUKKEY

ECLTON C THOMAS PROF

PAV EOX 360

RICHNOND HILL

CNT $L 4 C 4 Y 6$

CANACA

CCNALZOLA S DR

CES DE MEUDON

FRANCE
BLINDV N S DR

STERNBERG STATE AS-

117234 MOSCOW

U.S.S.R.

BLUM EMILE-JACQUES OR

IRAM EMILE DES MARTYRS

F-38O26 GRENOBLE

FRANCE

BOBROVNIKOFF NICHOLAS T

OP

1623 VISALIA AVE

RERKELEY CA 94707

$U . S . A$.

BOEHM KARL-HEINZ PROF

ASTRONQMY DEPT

UN IVER S I TY OF

WASHINGTON

SEATTLE WA 98195

U.S.A.

BOEHME SIEGFR IED DR

ASTRONOMI SCHES

RECHEN INSTI TUT

MOENCHHOF STR $12-14$

D-6900 HE IDEL

GERMANY, F.R.

BOESGAARD ANN M PROF

INST FOR ASTRONOMY

2680 WOODLAWN OR

HCNOLULU HI 96822

U.S.A.

BOGGESS NANCY W DR

NASA HEADQUARTERS

WASHINGTCN DC 20546

U.S.A.

EOHL IN RALPH C DR

CODE 672

GODDARO SPACE

FLIGHT CENTER

GREENBELT MD 20771

U.S.A.

BOK BART J PROF

STEWARD OBSERVATORY

UN IVER SI TY AR I ZONA

TUCSON AZ 85721

$U . S . A$.

BOLDT ELIHU DR

CODE 661

GODDARD SPACE FLIGHT

CENTER

U.S.A.

BOLTON JOHN G

ANRAO

BOX 276

PARKES NSW 2870

AUSTRALIA

BOND HOWARD E DR

DEPT PHYSICS AND AST

LOUISIANA STATE

UN IVER SITY

BATON ROUGE LA 70803

U.S.A.
BLITZSTEIN WILLIAM DR

DEPT OF ASTRONOMY

UNIV OF PENNSYLVANIA

D RITTENHOUSE LAB EI

PHILADELPHIA PAI9174

U.S.A.

BLUM PETER PROF

INST F ASTROPHYSIK

UNI VERSI TAET BONN

AUF DEM HUEGEL 71

D-530D BONN I

GERMANY, F.R.

BOCHONKO DONALD R DR

DEPT MATH ASTRONOMY

UNIV OF MANITOBA

WINNI PEG MB R3T 2 N2

CANADA

BOEHM-VITENSE ERIKA PROF

ASTRONOMY DEPT

UNI VERSITY OF

WASHINGTON

SEATTLE WA 98195

U.S.A.

BOERNER GERHARD DR MPI FUER PHYSIK UND ASTROPHYSIK

FOEHR INGER RING 6

D-8000 MUENCHEN

GERMANY, F.R.

BOESHAAR GREGORY ORTH DR

DEPT OF PHYSICS

UNIV OF OREGON

EUGENE OR 97403

U.S.A.

BOGORODSKIJ A F DR

KIEV STATE UNIVERS I -

TY ASTRONOMICAL

OBSERVATORY

$252053 \mathrm{KIEV}$

U.S.S.R.

BOHRMANN ALFRED PROF

SCHAERSTRASSE 23

GERMANY, F.R.

BOKSENBERG ALEC DR

UNIV COLLEGE LONDON

GOWER STREET

LONDON WCIE 6BT

U.K.

BOLEY FORREST

WILDER LABORATORY

DARTMOUTH COLLEGE

HANOVER NH 03755

U.S.A.

BONANOMI J DR

QBSERVATOIRE

DE NEUCHATEL

CH-2OOO NEUCHATEL

SWI TZERLAND

BONDARENKO L N DR

STERNBERG STATE AS-

TRONOMICAL INSTI ITUTE

117234 MOSCOW

U.S.S.R. 
ECNE 1 HERMANN PROF SIR LEPT OF ENERGY MILLPA:NK

LCNCCN SWIP $40 \mathrm{~J}$

U.K.

PCANET ROGER M CR LPSP CNRS

F-9 137 U VERRIERES

LE GUISSON

BCNCV $\triangle N G E L$ DR

ASTRCVEMICAL

C. ESERVATOFY

$P$ C PCX 36
15 SUIA 4

EULGARIA

PCCTH RCY 5 CR

NUFFIELD RADIO ASTRO

NCNY LAPORATORIES

JCLRELL BANK

NACCLESFIELD SK119CL

U.K.

$P C R G M I N C$ J DR
DEPT D ASTROPHYS I QUE

INAIVEZSITE DE NICE

PARC VALRCSE

$F-16: 34 \mathrm{NICE}$ CEDEX

FRANCE

BCSNCN-CPESPIN CENISE

bic 33

$E-\overline{4} 00^{3} L I E G E$

EELOIUM

SCTTINELLI LUCETTE DR

UE VEUCON

UAE I CASTRONOMIE

FROACE MEUDON

BCULCV JACQUES J RR

El AVENUE: DE

$L$ CHSERVATOIRE

F-75OIL PARIS

FPANCF

ECUVIER PIERRE PRDF

CFSERVATOIRE

OE GEVEV

CH-1290 SAUVERNY GE

SWITIERLAND

BCWYEK C STUART PROF

UNIVERS ITY

CF CALIFORNIA

ASTRL NONY DEPT

BESK LEY CA 94729

L. .

SCYCE PFTER P CR

164 HCBART ST

G. $A$ HIVGTON DC 20009

U.S.A.

13CZIS GEORGE PROF

LEPT THEOQ MECHANICS

UAIVLRSITY

CF THESSALONIKI

THESSALCNIKI

GRETCE
BONEV T MR

OES POPULAIRE

BUL GAR IA

BONNOR W B PROF

QUEEN EL I ZABETH

CALLEGEN HILL ROAD

LONDON WB 7AH

U.K.

BONSACK WALTER K PROF

INST FOR ASTRONOMY

2680 WOODLAWN DR

HONOLULU HI 96822

$U \cdot S \cdot A$.

BOPP BERNARD W DR

DEPT PHYSICS ASTRON

UNIV OF TOLEDO

TOLEDO OH 43606

U.S.A.

RORRA ERMANNO F DR

DEPT DE PHYSIQUE

UNIVERSITE LAVAL

STE FOY

QUEBEC PQ GIK 7P4

CANADA

BOTEL HEIRO A OR

LISBOA

PORTUGAL

BOUGERET J L DR

OBS DE MEUDON

FRANCE

BPURY AR SENE J DR

ITST D ASTROPHYSI QUE UPIVERSITE DE LIEGE B-4200 COINTE DUGREE BELGIUM

BOWELL E L G DR

LOWELL OBS

FLAGSTAFF AL 86002

U.S.A.

BOYARCHUK A A DR CR IMEAN ASTROPHYSICAL OBSERVATORY USSR ACAD UF SCI NAUCHNY 334413 CR IMEA

U.S.S.R.

BOYD ROBERT L F PROF MULLARD SPACE

SC IENCE LAB UCL HOLMEURY ST MARY U.K.

BOZKURT SUKRU DR

EGE UN IVERSITY

DESERVATORY

PK 21

BORNOVA IZMIR

TUPKEY
BONIFAZI ANGELO DR NOMICO

I 401 DO BOLOGNA

BONOMETTO SILVIOA DR

ISTITUTO DI FISICA

VIAAMARZOL

I 35100 PADOVA

TALY

BOOKMYER BEVERLY B DR DEPT OF PHYSICS AND ASTRONOMY CLEMSON UNI VERSITY CLEMSON

BORGMAN JAN DR PROF KAPTEYN OBSERVATORY MENS I-NGHEWEG 20 RODEN DR

THE NE THER LANDS

BOSMA PIETER B DR SUBFAC NATUURKUNDE VRI JE UNIVERSI TEI

DE BOELELAAN $10 B 1$

THE NETHERLANDS

BOTEZ ELVIRA DR

INSTI TUT D.ENSEIGNE-

MENT SUPERI EUR

5800 SUCEAVA

ROUMANIA

\section{BOUIGUE ROGER PROF \\ LAB D ASTRONOMIE \\ UNI VERSI T \\ PAUL SABATIER \\ F-3IO77 TOULOUSE COX \\ FRANCE}

BOUSKA JIRI DR

DEPT OF ASTRONOMY

CHARLES UNIVERSITY

SVEDSKA

150 OO PRAHA

CZECHOSLOVAKIA

BOWEN EDWARD G DR $50 I O$ MAXWELL AVENUE U.S.A.

BOYARCHUK MARGARITA E OR CRIMEAN ASTROPHYS

OBSERVATORY

P IO NAUCHNYJ

334413 CRIMEA

U.S.S.R.

BOYTON P E DR

UNIV OF WASHINGTON

SEATTLE WA 98195

U.S.A.

BRACCESI ALESSANDRO PROF

OSSERVATORI

ASTRONOMICO

VI A ZAMBONI 33

$1-40126$ BOLOGNA

ITALY 


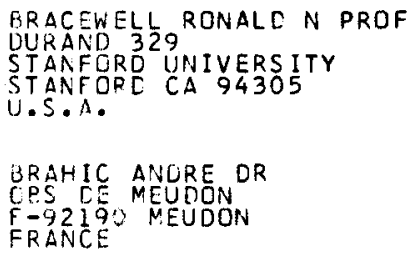

BKANSOA NICHOLAS J B A DR GENERAL BOARD OF THE FACULTIES

THE CLD SCHOOLS

I).K.

ERAUNINGER H DR MPI FUER PHYSIK UND $\triangle S T R C P H Y S I K$

INST EXTRATERR PHYS

[- 8 SO GARCHING

GERNANY, F.R.

GREALEY G A P ENG

ICAL OBSERVATORY

$507 \mathrm{I}$ W SAANICH RD

VICTCR IA BC V $8 \times 3 \times 3$

CANAEA

EKECKINRIDGE JAMES B DR

$J P L$ CALTECH

NAIL STOP IBZ-30

4800 DAK GROVE CR

PAS ADENA CA 91103

U.S.A.

BREJCC IZABELLA I DR

PULKCVC OBSERVATORY

19614 LENINGRAD

U.S.S.R.

RRIEVA EOUARDO PRUF

OBSERVATORIO

NACI CUAL

UCGCTA

UCGCTA
CELCMEIA

DRINKMAN BERT C DR

SPACE RESEARCH LAB

DENELUXLAAN 21

UTRECHT

THE NETHERLANDS

THCGLIA PIETRD DR

CSSERVATORIO

ASTRONOMICO

I- 220155 MERATE COMO

I TALY

FRCSCHE PETER PROF

CESERVATORIUM

HCHER LIST

D-5508 DAUN

GEKMAVIY, F.R.
BRAES L L E DR

STERREWACHT

POSTBUS 9513

2300 RA LEIDEN

THE NETHERLANDS

BRANCH DAVID DR

DEPT OF PHYSICS AND

ASTRONOMY

UN IVERSITY OKLAHOMA

NORMAN OK 73019

U.S.A.

BRANDT JOHN C DR

CODE 680

GODDARD SPACE

FL IGHT CENTER

GREENEELT MO 20771

U.S.A.

BRAUDE SEMION YA PROF AG

INST RADIOPHY ELECTR

ACADEMY OF SCIENCES

THE UKRAINIAN SSR

310085 KHARKOV

U.S.S.R.

BRAUNSFUR TH EDWARD PH D

ASTRONOMI SCHES INST

RUHR UN I VER S I TAET

POSTFACH 102148

D-4630 ROCHUM

GERMANY, F.R.

BRECHER AVIVA DR PRDF

DEPT OF PHYSICS

WELLESLEY COLLEGE

WELLESLEY MA 0218

$U . S . A$.

BREGER MICHEL DR

DEPT OF ASTRONOMY

UNIVERSITY OF TEXAS

AUSTIN TX 78712

U.S.A.

BRETAGNON $P$ DR

BUREAU D LONGI TUDES

77 AVENUE DENFERT-

ROCHEREAU

F-750 14 PAR IS

FRANCE

BR IHAYE CHARLES C A DR

SCHEMER INGLAAN $14 \mathrm{~A}$

1900 OVER I JSE

BELGIUM

BROADFOOT A LYLE DR

3625 EAST AJO WAY

TUCSON AZ 85713

$U \cdot S \cdot A$.

BROMAGE GORDON E DR ASTROPHYSICS RES DIV $S R C$ APPLETON LAB

CULHAM LAB

$\triangle B I N G D O N$ OXON

$\mathrm{U} \cdot \mathrm{K}$.

BROSTERHUS E B F DR

DOMINION ASTRO-

PHYSICAL OESERVATORY

5071 W SANICH RDRR7

VICTOR IA BC V $8 \times 3 \times 3$

CANADA
BRAHDE ROLF DR

INSTI TUTE OF THEORE-

UNI VERSITY OF OSLO

BLINDERN OSLO 3 NORWAY

BRAND PETER W J L DR

DEPT OF ASTRONOMY

UNI VERSITY

OF EDINBURGH

EDINBURGH EH8 $9 Y L$

U.K.

BRANSCOMB L M DR

NATI ONAL BUREAU OF

STANDARDS

WASHINGTON DC 20025

U.S.A.

BRAULT JAMES W DR

KITT PEAK NATI ONAL

OB SERVÁTORY

PO BOX 26732

TUCSON A2 85726

U.S.A.

BRAY ROBERT J OR

CSIRO NATI ONAL MEASU

REMENT LABORATORY

PO BOX 218

LINDFIELD NSW 2070

AUSTRALIA

BRECHER KENNETH PROF

MASSACHUSETTS INSTI -

TUTE OF TECHNOLOGY

ROOM 6-201

CAMBRIDGE MA 02139

U.S.A.

BRE I NHORST ROBERT A DR

ASTRON INSTITUT

STERNWARTE

AUF DEM HUEGEL 71

D-5300 BONN I

BRIDLE ALAN H PROF

DEPT OF PHYSICS

QUEENS UNIVERSITY

KINGSTON ONT KTL $3 N 6$

CANADA

BRINI D PROF

LABDRATORIO TESRE

VIA CASTAGNOLI 1

I -40100 BOLOGNA

BRODERICK JOHN DR

PHYSICS DEPT

VPI AND SU

BLACKSBURG VA 24061

U.S.A.

BROOKES CLIVE J DR

1 RYEF IELD CLOS

WEST HAGLEY

W MIDLANDS DYG OJS

U. K.

BROTEN NORMAN W

ASTROPHYSICS

NAT RES COUNC CANADA

OTTAWA ONT KIA ORG

CANADA 


\section{ERCUCKE ROGER DR 72.3 KUNNING ROPE CIRCL \\ PRCDINE I AN W A DR NUFFIELD RADID ASTRO NCNY LAEORATORIES JCLKELL EANK MACCLESFIELD SK119DL $\mathrm{U} \cdot \mathrm{K}$. \\ CRUCK HERMANN A PROF CEAIGOWER \\ PENICUIK EH26 9LA U.K.}

BRUMUEKG VICTOR A OR INST ITUTE FOR THEORE IICAL ASTRONOMY

$192187 \mathrm{~L}$

LRUAK WILLL IAM EE LR AASA HEADOUARTERS 405 MARYLAND AVES W WASHINGTEN DC 20546 U.S.A.

ERUZEK ANTON DR KIEP N NHEUER INSTITUT FUER SCNNENPHYSIK D-78US FREIBURG GERNAIUY, F.R.

EUES IRMELA O LR RENEIS STERNWARTE, C-86JD BAMRERG

GERNANY, F.R.

OULLARD EDWARE SIR

IGFP $A O 25$

UNIVERSITY DF

CALIFORNIA

LA JOLLA CA 92093

U.S.A.

EUNNER A N DR

PERKIN-ELMER CORP

MOS 846 WCSTER HTS RD

UANEURY CT OGB10

U.S.A.

EURCH CECIL R DR

PHYS ICS DEPT

THE UNIVERSITY

ERISTOL 8

U.K.

BURGESS ALAN CR

DEPT OF APPLIED MATH AND THEORET ICAL PHYS

SILVER STREET

CANBRIOGE CB3 9EW

$U . K$.
BROUW W N DR

RADIOSTERRENWACHT

OUDE HOOGEVEENSE

DIJK 4

DWINGELOO 7514

THE NETHERLANDS

BROWN ROBERT L DR

NATIONAL RADI

ASTRONOMY IOAS

BOX 2 EREN EANK WV 24944

BROWNLEE DONALO E PROF

DEPT OF A STRONOMY

UNIV CF WASHINGTON

SEATTLE WA 98195

U.S.A.

BRUCK MARY T DR

ROYAL DBSER VATORY

EOINBURGH EHS 3HJ

U.K.

BRUNER EL $C$ JR OR

LOCKHEED P A RES LAB

DEPT 52-12 BLDG 202

3251 HANO VER ST

PALO ALTD CA 94304

U.S.A.

BRUSTON P DR

$B$
$F-91370$

LE-BUISSON

BUCHLER J ROBERT PROF DEPT OF PHYSICS AND ASTRONOMY

UNIVERSITY FLORIDA

GAINESVILLE FL 32611

U.S.A.

BUFF JAMES $S$ DR

CEPT PHYSICS ASTRON

DARTMOUTH COLLEGE

HANOVER NH 03755

U.S.A.

BULLEN K E PROF DEPT DF APPLIED MATH UNIVERSITY OF SYDNEY SYDNEY NSW 2006

AUSTRALIA

BURBIDGE E MARGARET PROF

DEPT OF PHYSICS

UNIV OF CALIFORNIA

SAN DIEGO MCCO-II

LA JOLEA CA 92093

U.S.A.

BURGER J J DR IR WERK GROEP KOSM I SCHE STRAL ING K ONNES LAB NIEUWSTEEG 18

LEIDEN

THE NETHERLANDS

BURGESS DAVID DDR

BLACKETT LABORATORY

IMPER IAL COLLEGE OF

SCI AND TECHNOLDGY

LONDON SW7 $28 Z$

U.K.
BROWN HARR I SON DR

DIV OF GEOLOG ICAL

SCI ENCES

CALTECH

PASADENA CA 91103

U.S.A.

BROWN RONALD D PROF

CHEMI STRY DEPT

MONASH UNI VERSI TY

WELLING TON ROAD

CLAYTON VICT 3168

AUSTRALIA

BROWNLEE ROBERT $R$ DR

G-DO MS 570

LOS ALAMOS SCI

LABORATORY

LOS ALAMOS NM 87544

U.S.A.

BRUECKNER GUENTER E DR

CDDE 7142

NAVAL RESEARCH

LABORATORY

WASHINGTON DC 20375

U.S.A.

BRUNET JEAN-PIERRE DR

OBSER VATOIRES

1 AVE C FLAMMARION

F-3ISOO TOULOUSE

BRUWER J A

SOUTH AFRICAN ASTRONOMICAL OBSERVATORY

$P O B O X 3718$

JOHANNESBURG 2000

BUDDING EDWIN DR

ASTRONOMY DEPT

UNIVERSITY OF

MANCHESTER

MANCHESTER MI3 9PL

U.K.

BUHL DAVID DR

INFR RADID ASTRO BR

NASA GODDARD SPACE

FLIGHT CTR CODE 693

GREENBELT MD 20771

U.S.A.

BUMBA VACLAV DR

ASTRONOMICAL INST OF

ACADEMY OF SCIENCES

25165 ONDRE JOV

CZECHOSLOVAKIA

BURB IDGE GEOFFREY R PROF

OFF OF THE DIRECTOR

KITT PEAK 2673

TUCSDN AZ 85726

U.S.A.

BURGER MARI JKE DR

SPARRENSTRAAT 58

1170 BRUSSEL

BELGIUM

BURKE BERNARD $F$ DR

DEPT OF PHYSICS

CAMBRIDGE MA 02139

U.S.A. 


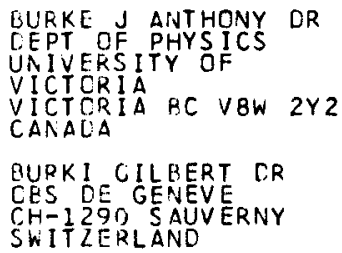

CABRITA ELEQUIEL CR CESERVATORIO
DE LISHCA
TAPACA LISBOA
PCRTUGAL

CACCIN BRUNO CSSERVATORIO ASTRONO NICE OI CAPOOIMONTE $\checkmark I A$ POIARIELLO IG I-E: 131 NAPOLI I TALY

CALAVAI G PROF ESSERVATORIO ASTRO FISICO DI ARCETRI ITALY

\section{CALCI VITTORIA DR \\ ASTRCF IS ICA SPAZ IALE C F 67 ROMA I -00 44 FRASCAT I T ALY} CANARE BAS BADIA V AOS PROF FACULTAC DE CIENCIAS UAIV DE ZARACOZA

$Z \triangle R \triangle G O Z A$

SFAIN
BURKHART C DR

OES DE LYON FRANCE.

BURLAGA L F DR

NASA GSFC

GREENBELT MD 20771

U.S.A.

BURSA MILAN DR

ASTRONOMICAL INST

CSAV

BUDECSKA 6

120 23 PRAHA 2

BUSCOMBE WILLIAM PROF

ASTRONOMY DEP

NORTHWESTERN

UN IVER SITY

EVANSTON IL 60201

U.S.A.

BUTLER C JOHN DR ARMAGH DBSER VA TORY ARMAGH BTGI 9DG

U.K.

BYKOV M F DR

ASTRONOMICAL INST UZBEK IAN ACADEMY OF SCIENCES

7 COOOO TASHKENT

U.S.S.R.

BYSTROV NIKOLAI F DR

PULKOVO OBSERVATORY

196140 LENINGRA

U.S.S.R.

CACC IAN I ALESSANDRO DR OSSERVATORIO ASTRONOMICO DI ROMA I - 00100 ROMA

ITALY

CADEZ ANDREJ DR

UNIV OF LJUELJANA

JADR AN SKA 19

61000 LJUBL JANA

YUGOSLAVIA

CALAME ODILE DR

AVENUE COPERNIC

F-O6130 GRASSE

FRANCE

CALVANI MASSIMO DR OSSERVATOR IO A STRONDM ICO ITALY 30 O PADOVA

CAMERON ALASTAIR G W PROF HARVARD COLLEGE

60 GARDEN STREET

CAMBR IDGE MA 02138

U.S.A.
BURKHEAD MART IN S

ASTRONOMY DEPT

I NDI ANA UNIVERSITY

SWAIN HALL WEST

BLOOMINGTON IN 47405

U. S.A.

BURNELL SUSAN J B DR MULLARD SPACE SC LAB LONDON

U. K.

BURTON $W$ BUTLER DR

DEPT OF ASTRONOMY

UNIV OF MINNESOTA

116 CHURCH ST S E

MINNEAPOLIS MN 55455 U.S.A.

BUSER ROLAND DR

ASTRONOMICAL INST

UNIV OF BASEL

VENUSSTRASSE ?

CH-4 I 22 BINNI NGEN

SWI T ZERLAND

BUTLER DENNIS DR

YALE UNIV

BOX 2023 YALE STAT

NEW HAVEN CT O6520

U.S.A.

BYRD GENE G DR

DEPT PHYSICS ASTRON

UNIV OF ALABAMA

BOX 1921

UNIVERSITY AL 35486

U.S.A.

BYSTROVA NATALIJA V DR

SPECIAL ASTROPHYSI -

CAL OB SERVATORY

LENI NG RAD BRANCH

196140 LENINGRAO

U.S.S.R.

CACCI ANI CARLA DR

IST OI ASTRONOMI A

I 401 OOO BOLOGNA

CAHN JULIUS H ASSOC PROF

UNI VER IITY OF

ILLI NOI S OBSERVATORY

URBANA IL 61 BO

U.S.A.

CALLEBAUT DIRK K DR UNI VERS I TE I TSPLEIN I B 2610 WILRIJK-ANTW BELGIUM

CALVO MANUEL

DEFPTO DE AS TRONOMIA

UNI VERSIDAD ZARAGOZA

ZARAGOZA 9

SPAIN

CAMERON WINIFRED S MRS

CODE 601

GODDARD SPACE FLIGHT

CENTER

GREENBELT MD 20771

U.S.A. 
CANICHEL HENRI CR

CRSERVATOIRE

IE TCULOUSE

T-3.5ST TOULOUSE

FRANCE

CANPBELL DONALDB

ARECIBC OHSERVATDRY

PCX $\rightarrow 95$

AKECIHC PR 00612

U.S.A.

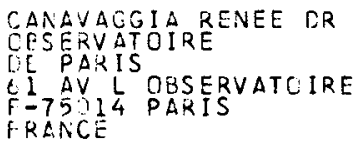

CANIZARES CLAUDE R PROF IIT

RN 37-50

CANCRIDGE MA O2139

U.S.A.

CANNCY WAYNE H TE

DEPT OF PIIYS ICS

4700 KTELE STREET

CCWNSVIEW ONT MJJIP3

CANADA

CAPEN CHARLES F

LCWELL OBSERVATOKY

ECX 1269

FLAGSTAFF AZ 86001

U.S.A.

CAPRIOTTI EUGENE R CR

CEPT SF ASTRONOMY

5058 A SMITH LAB

CCLUVBUS OH 43210

U.S.A.

CARDCNA OCTAVIO OR

INST NAC DE ASTROFIS

APCC PCSTAL 216

FUEBLA PUE

NEXICO

CARLETCV NATHANIEL P OR

SICAL CBSERVATORY

GO GARCEN ST

CAMERILGE MA U2138

U.S.A.

CARCFF LAWRENCE J

AVES RES CTR MS245-3

NCFFETT FIELD

U.S.A.

CARR THOMAS D PRUF

DEPT OF PHYS ICS AND $\triangle S T R C N D M Y$

UNIVERSITY FLORIDA

GAINESVILLE FL 32611

U.S.A.

CARRASCC GUILLERMO DR

CES ASTRON NAT IONAL

UNIVERSIOAO DE CHILE

CASILLA 36-D

SANT I AGC

CHILE
CAMM G LESLIE DR

THE UNIVERSITY

MANCHESTER MI3 SPL

U.K.

CAMPBELL JAMES W

BLACKFORD HILL
EDINBURGH EH9 $3 H J$

U.K.

CANDY MICHAEL P MR

PERTH OB SER VA TORY

WALNUT ROAD

BICKLEY 6076

AUSTRALIA

CEANNDN C J DR

MATHEMATICS

UN IVERSITY OF SYDNEY

SYONEY NSW 2006

AUSTRALIA

CANTU ALBERTO M DR

OSSERVATOR IO ARCETRI

LARGO FERMI 5
I -50125 FIRENZE

IT -50125 FIRENZE

CAPLAN JAMES

MAR

PLACE ILLE VERRIER

PLACE LE VARR IER

FRANCE

CAPUTO FILIPPINA DR

CNR ASTROF I SICA

ROMA

I=O0O44 FRASCATI

CARDUS ALMEDA J DR
OBSER VATOR ID DEL

EBRO

RDQUETAS

TOR TOSA BARCELONA

SPAIN

CARLQVIST PER A OR

ROYAL INST OF

TECHNOLOGY SWEDEN

CAROUBALOS C A PROF LAE ELECTRONIC PHYS UN IVER ITY OF A THENS 104 SOLONOS STREET ATHENS 144

CARRANZA G J DR

LAPR IDA

COR DOBA

CARRASCO LUIS DR

INST I TUTO DE

ASTRONOMIA

APDD POSTAL 70264

MEXICO 20 DF

MEXICO
CAMPRELL BRUCE DR

CFHT CORPORATION

BCX 1597

KAMUELA HI 96743

U. S. A.

CANAL RAMON M DR

DPTO FIS TIERRA COS

UNI VERSIDAD

DE BARCELDNA

BARCELONA 14

SPAIN

CANF IELD RI CHARD C DR PHYSICS C-OII UCSD

U.S.A.

CANNON RUSSELL D DR

ROYAL OBSERVATORY

BLACKFORD HILL

EDINBURGH EH9 $3 \mathrm{H}$ J

U.K.

CAPACCIOLI MASSIMO DR

ASTRONOMICAL OBS

UNIVERSITY DF PADOVA

ITALY 350 PADOVA

CAPRIOLI GIUSEPPE PROF

DSSERVATORIO ASTRONO

MICO DI ROMA

VIAA TRIONFALE 204

RQMA

CARBON DUANE F DR

KITT PEAK NAT OBS

P $O 0 \times 26732$

TUC SON AZ 85726

U.S.A.

CARESTIA REINALDO A DR

OBS ASTRON F AGUILAR

AV BENAVIDEZ 8175

OESTE RIVADAVIA

5400 SAN JUAN

ARGENTINA

CARM LAWRENCE E DR

SACRAMENTO PEAK OBS

CARPENTER LLQYD DR

CENTER

CODE 921

GREENBELT MD 20771

U.S.A.

CARRASCO GARRORENA R DR CLARA DEL REY 14 SPAIN

CARROLL P KEVIN PROF

PHYSICS DEPT

UNI VERSITY COLLEGE

BELFIELD

IRELAND 


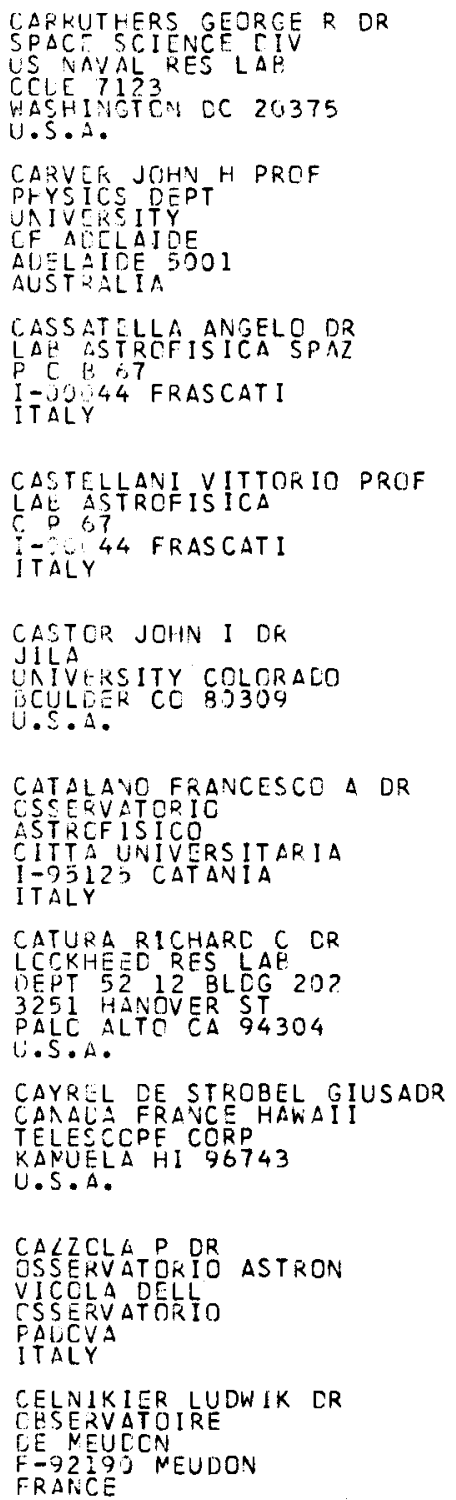

CESARSKY CATHERINE J CR SEP-CEN SACLAY R $P$ NO 2 F-91190 GIF-YVETTE FRANCE

CESTER HRUNO PROF CSSERVATORIO I IA TI I PQLOLIL

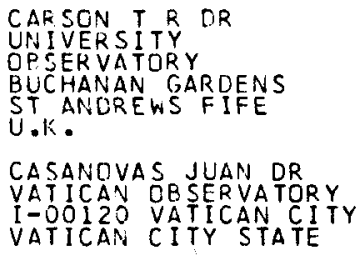

CYASSE M DR CEN SACLAY

B P NO 2 GIF-S-YVETTE

CASTELLI FIDRELLA OR NOMICO

VIA TIEPOLO 11

ITALY 34131 TR IESTE

CASWELL J L DR

CSIRO RADIOPHYSICS

OBOX 76

EPPING NSW 2121

CATALANO SANTO DR

IST CI ASTRONOMIA

CITTA UN IVER SI TAR I A

ITALY

CAUGHLAN GEORGEANNE R

PROF

DEPT OF PHYSICS

MONTANA STATE UNIV

BOZEMAN

CAYREL ROGER DR

CANADA FRANCE HAWAII

TELESCOPE CORP

KAMUELA HI 96743

U.S.A.

CECCARELLI M PROF

ISTITUTO DI FISICA

GENERALE

UN IV DI BOLOGNA

ITALY 100 BOLOGNA

CEPLECHA ZDENEK OR

ASTRONOMICAL INST

CZECHO ACAD SC IENCES

25165 ONDREJOV

OASER VATORY

CZECHOSLOVAKIA

CESARSKY OIEGO A DR

DESERVATOIRE

DE MEUDON

RAOIO ASTRONOMIE

F-92190 MEUDON

FRANCE

CHAFFEE FREDERIC H DR MT HOPK INS

OBSER VATORY

P 0 BOX 97

$\triangle M A D O \quad A Z 85640$

U.S.A.
CARSWELL RDBERT F DR

INST OF ASTRONOMY

MAOINGLEY ROAD

CAMBRIDGE CB3 OHA

U.K.

CASINI CATERINA DR

ISTI TUTO DI

ASTRONOMIA

VIA BRERA 28

CASSINELLI JOSEPH P DR

NOMY DEP

UNI VERSI TY WISCONS IN

MADISON WI 53706

U.S.A.

CASTELLI JOHN P

HANSCOM AFB MA 01731

U.S.A.

CATALA ASUNCION P DR

DPTD FIS TIERRA COS

UNI VERSIDAD

DE BARCELONA

BARCELONA 28

SPAIN

CATO TORGNY DR

RODDBAATSGATAN 3

$S-42171$ VAESTRA

FROE LUNDA

SWEDEN

CAVALIERE ALFONSO G PROF IST DI ASTRONOMIA VIC OSSERVATORI

CAZENAVE ANNY DR

CNE S-GRGS

II AVE EDQUARD BELIN F-31400 TOULOUSE

CEFOLA PAUL J DR

MAIL STATION 64

C S DRAPER LAB CAMBRIDGE MA O2I39 U.S.A.

CEPPATELLI GUIDO DR OSSERVATORIO ASTROFISICO DI ARCEIRI I 50 ITALY FI RENZE

CESCO CARLOS DR OBS ASTR F AGUILAR BENAVIDEZ 8175 DESTE 5407 MARQUESADO SAN JUAN

CHAISSON ERIC J PROF HARVARD OBSERVATORY CAMBRIDGE MA O2138

U.S.A. 
CHANARAUX P DR CPS DE MEUDON FRANCE

CHAMBLISS CARLSON R DR KUTZTCWN STATE

CLLLESE

KUTZTOWN PA $1953 i$

U.S.A.

CHAPMAN CLARK R CR

PLANETARY SC INST

SUITE 2 O I

TUCSCN $2 \frac{1}{2} 85719$

U.S.A.

CHAPRONT J DR
BUREAU C LONGITUDES
77 AVE CENFERT -

RCCHEREAU

FT5 14 PAR IS

FRANCE

CrAU WAI Y PRDF

PHYSICS DEPT

GUEEYS UNIVERSITY

KINGSTCN CNT KTL 3NG

CHENG CHUNG-CHIEH DR

NAVAL RESEARCH LAE

CCLE 7174C

WASHINGTON DC 20375

U.S.A.

CHERNEGA IN A A CR

CBSERVATORY OF THE

KIEV UNIVERS ITY
CESEKVATORNAYA

$252553 \mathrm{KIEV}$

U.S.5.k.

CHEVALIER CLAUDE DR

CESERUUCLN

FRAN

CHICSI CESARE S DR

ISTITUTC EI

URIVFRSITA DI PACOVA

I-35IJO PADOVA

ITALY

CHITRE D M DR

RANA RESEARCH INST

$P C$ HEDEAL

IANOALCRE 560000

IACI $A$

CLIUIERI CLAUOIO PROF CSSERVATORIO ARCETRI

LARGÜ FERMI 5

ITATY $=55$ FIRENZE

CHMIELEWSKI YVES CR

UEPT CF ASTRONONY

$R^{2} L$ NOCRE HALL

AUSTINTX 78712

U.S. 2 .
CHAMEERLAIN JOSEPH M DR

ADLER PLANETAR IUM

1300 S LAKE SHORE DR

CHICAGO IL 60201

U. \$.A.

CHANDRASEKHAR S PROF

CHICAGO IL 60637

U.S.A.

CHAPMAN GARY A DR

DEPT PHYSICS ASTRON

CALL IF STATE UNIV

NORTHRIDCE CA 91324

U.S.A.

CHAPRONT- TOUZE M DR

BUREAU D LONGI TUOES 77 AVENUE DENFERT-

RCCHEREAU

F- 75014 PAR IS

FRANCE

CHAVIRA ENR IOUE SR

INST NAC ASTROPHYS

OP TICA Y ELECTRONICA

AP PUSTAL $216 Y 51$

MEXICO

CHEREDN ICHENKO V I DR

KIEV POLYTECHNICAL

INSTITUTE

$252056 \mathrm{KIEV}$

U.S.S.R.

CHERNYKH N S DR

CR IMEAN ASTRO-

PFYSICAL ORSERVATORY

NAUCHNY

334413 CR IMEA

U.S.S.R.

CLEVALIER ROGER A DR

DEPT OF ASTRONOMY

P O BOX 3818

CHARLOTTESVL VA 22903

U.S.A.

CHIS CHEORGHE PROF DR

STR REPUBLICI I 109

$3400^{\circ}$ CLUJ NAPOCA

ROUMANIA

CHITRE SHASHIKUMAR M DR

TATA INSTITUTE

HOMI BHABHA ROAD

COLABA

BOMRAY 400005

IND IA

CHIUDER I-URAGD F PROF

OSSERVATORIO ASTROFI

$S$ ICO DI ARCE TR I

$\angle A R G O E$ FERMI 5

I -50125 FIRENZE

CHCI BON CHOL

ACADEMY OF SCIENCES

PYONGYANG ASTRONOMI-

CAL OBSERVATURY

PYONGYANG

DEM PEOPLE'S REP OF KOREA
CHAMBERLAIN JOSEPH W PROF

DEPT SPACE PHYS ICS

AND ASTRONOMY

RICE UNIVERSITY

HOUSTON TX 77001

U.S.A.

CHANMUGAN GABESAR PROF

DEPT OF PHYSICS

AND ASTRONOMY

LA STATE UNIVERSITY

U.S.A.

CHAPMAN ROBERT D DR

CODE 680

GODDARD SPACE FLIGHT

GENTER

U.S.A.

CHARVIN PIERRE DR

INAG

77 AVENUE DENFERT

ROCHEREAU

F-75014 PARIS

FRANCE

CHEN KWAN-YU PRDF

DEPT OF PHYSICS AND

ASTRONOMY

UNI VERS I TY FLDR I DA

GAINESVILLE FL 32611

U.S.A.

CHEREPASHCHUK A M DR

STERNBERG STATE

A STRONOMICAL INST

NOMICAL INSTI TUTE

117234 MOSCDW

U.S.S.R.

CHERTOPRUD $V$ E DR

HYDROMETEOROLOG ICAL

CENTER OF THE USSR

123376 MOSCOW

U.S.S.R.

CHINCARINI GUIDO L DR

DEPT PHYS AND ASTRON

UNIV OF OKLAHOMA NEL

SON BD 440 W BROOKS

NORMAN OK 73069

U.S.A.

CHISTYAKOV V E OR

SOLAR STATION

692533 GORNOTAE ZHNOE

U.S.S.R.

CHIU HONG - YEE DR

MUDD BLOG RM 828

COLUMBIA UNIY

NEW YORK NY 10027

U.S.A.

CHLIETOVSKY FRANCA OR OSSERVATORIO ASTRO-

NOMICO

BRERA

MILANO

CHOLLET FERNAND

OBSERVATOIRE PARIS

GI A VENUE DE

$L$ OASERVATOIRE

F-750I 4 PARIS

FRANCE 
CHOPINET MARGUERITE DR CESERVATOIRE

CE BORLEAUX

F -33270 FLOIRAC

FRANCE

CHRISTIANSEN WILEUR PROF

SCHCOL OF ELECTRICAL

ENGINEERING

UNIVERSITY OF SYDNEY

SYCNEY NSW 2006

AUSTRALIA

CHUHB TALBOT A OR

$5023 \mathrm{~N} 38 \mathrm{TH} S T$

ARLINGTON VA 22207

U.S.A.

CHUPP ECWARD L CR

PHYS ICS DEPT

CENERITT HALL

UNIV NEW HAMPSHIRE

CURHAM NH 03824

U.S.A.

CHUVAEV K K DR

CQINEAN ASTROPHYSI-

CAL CBSERVATDRY USSR

ACAD OF SCI NAU

U.S.S.R.

CIO PALACIOS RAFAEL PROF FACULTAO DE CIENCIAS Z $\triangle R \triangle G O Z A$

SPAIN

CLARIA JUDR

DE ASTRCNOM I A

$\triangle P T$ DE CORREDS 264

$M E R I Q A$

VERELUELA

CLARK DAVID H DR

RCYAL GREENWICH

CESERVATORY

HAILSHAM

EAST SUSSEX BN27 IRP

A

CLARK THOMAS A CR

PACIC ASTRO BRANCH

NASA OCDDARD SPACE

GREEIVBLLT MD 20771

U.S.A.

CLARKE THOMAS R UR

REYAL ONTARIO MUSEUM

10 OUEENS PK CRESC

TCRCNTC ONT MSS $2 C 6$
CANADA

CLEGG PETER E OR

GUEEN MARY COLLEGE

LCNDCN EI $4 \mathrm{NS}$

U.K.

CLIFTON KENNETH ST DR

MASA E S 64 SALL SFC AL 35812

U.S.A.
CHOU KYONG CHOL PROF

DEPT OF A STRONOM

AND METEOROLOGY

YOUNSE I UNIVERSITY

SEOUL I20

REPUBLIC OF KOREA

CHR ISTY JAMES WALTER DR

EXPLDRATORY DEV ST

US NAVAL OBS

WASHINGTON DC 20390

U.S.A.

CHUGAJNOV P F DR

CRIMEAN ASTROPHYSI-

CAL OESERVATOR Y OS SR

334413 CRIMEA

U.S.S.R.

CHURCHWELL EDWARD B DR

WASHBURN ORSERVATORY

UNIVER SITY WI SCONSIN

475 N CHAR TER ST

U.S.A.

CHVOJKOVA WOYK E DR

ASTRONOMICAL INST

BUDEC SKA 6

12023 PRAHA 2

CILLIE G G PROF

4 MINSER IE STREET

STELLENBOSCH 7600

SOUTH AFR ICA

CLARK ALFRED JR PROF

DEPT MECH AND AERO

SCIENCES

UNIVERSITY ROCHESTER

ROCHESTER NY 14627

U.S.A.

CLARK FRANK OL IVER OR

DEPT PHYSICS ASTRON

UNIV OF KENTUCKY

LEXINGTON KY 40506

U.S.A.

CLARK THOMAS ALAN DR

PFYSICS DEPT

UNIV OF CALGARY

2920-24 AVENUE N W

CALGARY ALB T2N IN4

CANADA

CLAUSEN JENS VIGGO LEKTCR

CLPENHAGEN UN I VER

SITY OB SER VA TOR

BRORFELDE

DK-4340 TOLLOSE

DENMARK

CLEGG ROB IN F S DR

DEPT OF ASTRONOMY

UNIV OF TEXAS

AUSTIN TX 78712

U.S.A.

CLIMENHAGA JOHN L PROF

DEP I OF PHYSICS

UNIVER SITY

VICTORIA BC V8W $2 Y 2$

CANADA
CHRI STENSEN-DALSGAARD J

INST D'ASTROPHYS I QUE

UNIVERSITE DE LIEGE

AVENUE DE CDINTE 5

B-420

CHRISTY ROBERT F DR

PROVOST OFFICE

CALIFORNIA INSTITUTE

OF TECHNOLOGY

PASADENA CA 91125

U.S.A.

CHUN MUN-SUK DR

DEPT OF ASTROPHYSICS

SOUTH PARKS ROAD

OXFORD OXI $3 R Q$

U.K.

CHURMS JOSEPH

S A ASTRONOMICAL OBS $P$ POX 9 .

QB SERVATORY

CAPE 7935

SOUTH AFRICA

CIATTI FRANCO DR

QSSERVATORIO

ASTROFISICO

I 36012 ASIAGO VI

CIMINO MASSIMO A PROF

YIA A CAOLOLO 19

I TALY

CLARK BARRY G DR

NATI ONAL RADIO

ASTRONOMY OBS

$P$ O BOX $O$

SOCORRO NM 87801

U. S.A.

CLARK GEORGE W PROF

MIT ROOM 37-6I

CAMBRIDGE MA O2139

U.S.A.

CLARKE DAVID DR

DEPT OF ASTRONOMY

THE UNIVERSI TY

GLASGDW G12 $8 Q Q$

U. K.

CLAYTON DONALD D PROF

AND ASTRONOMY

RICE UNIVERSITY

HOUSTON TX 77001

U.S.A.

CLEMENT MAURICE J PROF

DEPT OF ASTRONOMY

UNI VERS I TY OF

TORONTO

TORONTO ONT MSS IAT

CANADA

CLUBE S $Y M$ MR

ROYAL ORSERVATORY

EDINBURGH EH9 $3 H J$

U.K. 
CCCHRAN WILLIAM CAVID DR

DEPT OF ASTRONOMY

AUSTIV TX 78712

U. S. A.

CCOINA LANDABERRY S J DR INST ITUTO ASTRONOMI-

CAIXA POSTAL 30627

IOOO SAO PAULO SP

PRAZIL

CCFFFEN DAVID L CR

$28 B O$ PROACWAY

NEW YORK NY 10025

U.S.A.

CCHEN JEFFREY M CR

PHYSICS DEPT

UAIV OF PENNSYLVANIA

PHILADELPHIA PAI9174

U.S.A.

CCHEN MARSHALL H PROF

CALTECH 102-24

PASACENA CA 91125

U.S.A.

CCLEURN DAVID S DR

AMES RESEARCH CENTER

MCFFETT FIELD

CA 94335

U.S.A.

CCLLIN-SQUFFRIN SUZY DR OBSERVATOIRE

CE NEIJCON

F-92190 MEUDON

FRANCE

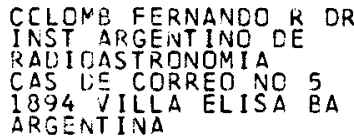

CCNCCNI PAOLO DR

CSSERVATORIO ASTRONCNICO

ERERA

MILAND

ITALY

CONNES JANINE CR

CIRCE

F-9144J ORSAY CEDEX

FRANCE

CCNTCPCULOS GEORGE PROF

ASTRGNOMY DEPT

PANEPIST IMIOPOLIS

$\triangle T H E N S 621$

GREECE

CCCK ALLAN F DR

SICS HCO-SAO

60 GARDEN ST

CAMBRIDGE MA 02138

U.S.A.
COCKE W JOHN PROF

STEWARO OB SERVATORY

UNIVERSITY AR I ZONA

TUCSON AL 85721

U.S.A.

CODINA VIOAL J M DR BARCELONA I3

SPAIN

COFFEY HELEN E DR

NOAA

NGSOC 063

BOULDER CO 80303

U.S.A.

CCHEN JUD ITH DR

KITT PEAK NATIONAL

OASERVATORY

P O BOX 26732

U.S.A.

COHEN MAR TIN DR

ASTRONOMY DEPT

UNIV OF CALIFORNIA

BERKELEY CA 94720

U.S.A.

COLE TREVOR W OR
CSIRO RADIOPHYSICS

P D BOX 76

EPP ING NSW 2121

AUSTRALIA

COLL INS GEORGE W I I PROF COLUMBUS OH 43210

U.S.A.

COLOMBO G PROF DR

UNIV DI PADOVA

VIA F MARZOLO 9

ITALY 3500 PADOVA

CONDON JAMES J DR

PFYSICS DEPT VA PQLY

325 ROBESON HALL

BLACKSBURG VA 24061

U.S.A.

CQNNES PIERRE DR

SERVICE D ASTR ONOMIE

B $P 1370$ VERRIERES LE

BUISSON

CONWAY ROBIN G DR

NRAL

JODRELL RANK

CHESHIRE SKII 9DL

U.K.

COOKE B A DR

$X$-RAY ASTRONOMY

GRDUP PHYSICS DEPT

THE UN IVER SI TY
LEICESTER LEI TRH

U.K.
CODE ARTHUR D

WASHBURN OBSERVATORY

UNIV OF WISCONS IN

MADISON WI 53706

U.S.A.

COELHO BALSA M C DR OBS ASTRONOMICO

COIMBRA

PORTUGAL

COGAN BRUČE C ${ }_{X-1}{ }^{2}$

LOS ALAMOS SC LAB

LOS ALAMOS SC LAB

U. S.A.

COHEN LEON PROF

DEPT OF PHYSICS

HUNTER COLLEGE

695 PARK AVE

NEW YORK NY 10021

U.S.A.

COHEN RAYMOND J DR

NRAL

JODRELL BANK

MACCLESF I ELD

CHESHIRE SKII 9OL

U.K.

COLGATE STIRLING A DR

THEORETICAL DIVISION

LOS ALAMOS SCIENTI-

FIC LAB MS 210

LOS ALAMOS NM 87845

U.S.A.

COLLINSON EDWARD H

THE COPSE

CHURCH LANE PLAYFORD

I PSWICH

SUFFOLK I P6 9DR

U. $K$.

COMBES M MR

OBS DE MEUDON

FRANCE

CONKLIN EDWARO K

C 15 MORTH INC

MANHATTAN BEACH

CA 90266

U.S.A.

CONTI PETER S DR

JILA

UNI VERSITY COLORADO

BOULDER CO 80309

U.S.A.

COOK ALAN H PROF

CAVENDISH LABORATORY

MADINGLEY RD

CAMBRIDGE CB3 OHE

U.K.

CORBIN THOMAS ELBERT DR

US NAVAL OBS

TRANSIT CIRCLE DIV

WASH I NG TON DC 20390

U.S.A. 


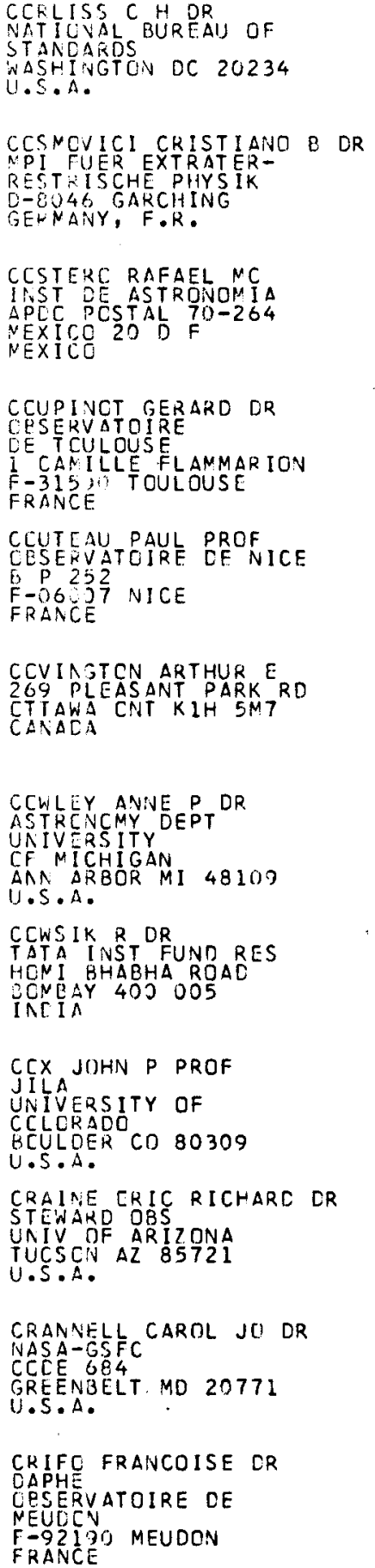

CORNEJO ALEJANDRO A MSS INADE POSTAL 216 Y 51 PUEBLA PUE

MEXICO

COSTAIN CC C DR

NAT RES COUNCIL

OTTAWA KIA ORG

CANADA

COUDERC PAUL DR

5 SOUARE DELAMB
F-75O14 PARIS

FRANCE

COURTES GEORGES PROF

OBSERVATOIRE DE

MARSEILLE

2 PLÁCE LE VERRIER

F- 13004 MARSEILLE

FRANCE

COUTREZ RAYMOND A J PROF

UNIVERSITE LIBRE DE

BRUXELLES

$50 A V$ F D ROOSEVELT

$B-1050$

BELGIUM

COWAN JOHN J DR

HARVARD SMI THS OBS

CTR FOR ASTROPHYSICS

60 GAREEN STREET

CAMBRIDGE MA 02138

U.S.A.

COWLEY CHARLES R PROF

ASTRONOMY DEPT

UN IVERSITY

OF MICHIGAN

ANN ARBOR MI 48109

U.S.A.

COX AR THUR N DR

LOS ALAMOS

SCIENTIFIC LAB

$P$ BOX $1 \in 63$

LOS ALAMOS NM 87545

U.S.A.

COYNE GEORGE $\checkmark$ DR

LUNAR AND PLANETARY

LABORATORY

UNIVERSITY ARI ZONA

TUC SON AZ 85721

$U \cdot S \cdot A$.

CRAM LAWRENCE EDWARD DR

SACRAMENTO PEAK OBS

SUNSPOT NM 88349

U.S.A.

CRAWFORD DAVID

KITT PEAK NATIONAL

OBSERVATORY

BOX 26732

IUCSON AZ 85726

U.S.A.

CR ISTALDI SALVATORE DR

OSSERVATORIO

ASTROF ISICO

CITTA UN IVER SI TAR I A

$1=95125$ CATANIA

ITALY
CORWIN HAROLO G JR DR

DEPY OF ASTRONOMY

UNIV OF EDINBURGH

EDINBURGH EHS $3 \mathrm{HJ}$

U. K.

COSTAIN CARMAN H DR

DOMINI ON RADIO

ASTROPHYSICAL OBS

$B O X 248$

PENTICTON BC V2A $6 \mathrm{~K} 3$

CANADA

COUNSELMAN CHARLES C PRDF

MIT DEPT EARTH AND

PLANE TARY SCIENCES

CAMBRIDGE MA O2139

U.S.A.

COUSINS A W J DR

$S$ A ASTRONOMICAL OBS

OBSERVATORY

OBSERVATORY

SOUTH AFRICA

COUTTS-CLEMENT CHRISTINE

DEPT OF ASTRONOMY

UNI VERSI TY TORONT O

TORONTO ONT MSS IAT

CANADA

COWIE LENNOX LAUCHLAN DR PR INCETON UNIV OBS

PEYTON HALL

PR INCETON NJ 08540

U.S.A.

COWLING THOMAS G PRCF

19 HOLLIN GARDENS

HEAD INGLY

LEEDS LS16 $5 \mathrm{NL}$

U.K.

COX DONALD P PROF

DEPT OF PHYSICS

UW MADI SON

1150 UNIVERSITY AVE

MADI SON WI 53756

U.S.A.

CRAIG IAN JONATHAN D OR

DEPT OF APPLIED MATH

UNIV OF WAIKATO

HAMI LTON

NEW ZEALAND

CRAMPTON DAVID DR DOMINION ASTROPHYSI CAL OBSERVATORY 5071 W SAANICH ROAD VICTORIA BC V $8 \times 3 \times 3$ CANADA

CRE ZE MICHEL DR BESANCON 41 AV $L$ OBSERVATOIRE F-25DOO BESANCON

CRISTESCU CORNELIA G DR OBSERVATOI RE

DE BUCAREST

75212 BUCAREST

ROUMANIA 


\section{CRCOM CAVID L DR \\ AFPLETCN LABORATCRY \\ DITTEN PARK \\ SLCUGH PERKS SL 3 $3 \mathrm{JX}$ \\ U.K.}

CFUISE ADRIAN MICHAEL DR MULLARC SPACE SC LAB UCRKING

SURREY RHS GNS U.K.

CSADA IMRE K DR KCAKELY OESERVATORY PCX to H-1525 BUDAPEST
HUNGARY

CUFFEY J MR N N STATE UNIV
DEPT OF EARTH SC AND
ASTRCINOMY

UNIV PK NM 88001

U.S.A.

CULHANE LEONARD DR MULLARE SPACE SC LAB HCLNHIJRY ST MARY CEKKING

SURR -Y RHS ONS

U.K.

CUAY YVETTE J DR

CESERVATOIRE CE

NEUDON

PLACE JANSSEN

$F=92190$ MEUDON

FRANCE

CZYZAK STANLEY J DR

ASTRCNOMY OEP

CHIC STATE UNIV

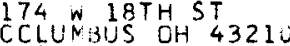

I..S.A.

CA RCICHA VIEIRA E CR

IAT UE FISICA

UAIV FECERAL DA

OUOO) PCRTO ALLECRE

URAZIL

DA SILVA LICIO DR

CESERVATORIO NACION

$R$ GEN GRUCE 586

$S$ CRISTCVAO

DoOOC RIO DE JANEIRO ERALIL

CAADIC ZARKO DR

YUGOS $\triangle C A D$ SC ART

CENET RCVA $1 B$

4102 ZAGREB

CANT zEE EOWARD J DR

NUFFIELE RADIO ASTRO

NCNY LABS

JCLRELL BANK

MCCLESFIELD SK1190L

U.K.

EALLAPCRTA N PROF

IST ITUTO ASTRONOMIA UNIVERSITA

VICLLO OSSERVATORIO

$I-35120$ PADOVA
CROVISIER J DR
OBS DE MEUDON
F-92I I9U MEUDON
FRANCE

CRUTCHER RICHARD M DR

UN IVERSITY OF

ILL INO IS OBSER VATORY

URBANA IL 61801

U.S.A.

CUDABACK DAVID D DR

RADIO ASTRONOMY LAB

UN IVER S ITY

OF CAL IFORNIA

BERKELEY CA 94720

U.S.A.

CUGNON PIERRE DR

OESERVATOIRE ROYAL

DE BELGIQUE

AVE CIRCULAIRE 3

BELGIUM

CULVER ROGER BRUCE DR

DEPT DF PHYSICS

COLDRADO STATE UNIV

U.S.A.

CUPERMAN SAMI PROF

DEPF OF PHYSICS AND

ASTRONOMY

TEL-AVIV UNIVER SI TY

RAMAT-AVIV

I SRAEL

D ANTONA FRANCESCA DR OSSERVATORIO ASTRONOM ICO ITOOIOO ROMA

DA SILVA A VC S OBS ASTRONUMICQ UN IV SANTA CLARA COIMERA

POR TUGAL

DACHS JOACHIM PROF DR ASTRONOMISCHES INST RUHR - UN I VER S I TAET POSTFACH 102148

D-4635 BOCHUM

DAHN CONAKD CURTIS DR

US NAVAL OBS

PLAGSTAFF AZ 86002

$U \cdot S \cdot A$.

DAISHIOO TSUNEAKI DR

CEPT OF SC IENCE

SCHOOL OF EDUCATION

WASEDA UNIVERSI TY

JAPAN

DALTABUIT ENRIQUE DR

INSTITUTO DE

ASTRONOMIA UNAM

APDO POSTAL 70264

MEXICO OF

MEXICO
CRUIKSHANK DALE P DR

2680 WOODLAWN DR

HONOLULU HI 96822

-U.S.A.

CRUVELLIER PAUL EDR

LAB ASTRO SPATI ALE

LES TROIS LUCS

F-13012 MARSEILLE

FRANCE

CUDWORTH KYLE MCCABE DR YERKES OBS

WILLIAMS BAY WI53191

U.S.A.

\section{CUGUSI LEONINO OR \\ IST DI ASTRONOMIA \\ UNI VERSITA \\ CAGLIAR \\ I TALY}

CUNNI NGHAM LELAND E PROF DEPT OF ASTRONOMY

UNIVERSITY OF

CALIFORNIA

BERKELEY CA 94720

U.S.A.

CURRIE DQUGLAS G OR

UNIV OF MARYLAND

COLLEGE PK MD 20742

U.S.A.

D'QDORICO SANDRO DR

OSSERVATORIO

ASTROFISICO

I 36012 ASIAGO VI

DA SILVA I F PROF DR FACULDADE DE CIENCIA LI SBON

DADAEV ALEKSANDR $N$ DR

PULKOVO OBSERVATORY

196140 LENINGRAD

U.S.S.R.

DAIGNE GERARD G

OBS DE MEUDON

FR92190 MEUDON

DALGARNO ALEXANDER PROF

HARVARD COLLEGE OBS

SO GARDEN STREET

U.S.A.

DAMBARA TAKESHI PROF SHI ZUOKA UNIVERS ITY OTANI SHIZUOKA 422 JAPAN 
CDNRY J M ANTHONY DR CEPT OF MATHEMATICS URRTHERARTYL RALEIGH NC 276,7 U.S.A.

CAPIZIGER I JOHN LR TELECCEPE PROJECT OR

CHIT? ILKLANDEVA 23

() $\triangle U T C D I J T$ G DR

7 ENTRTINST ASTROPHYS STERIWARTE BABELSE R LUX MEUFG STE ITA CDR-1', 2 PQTSDAM

G.C.R.

RAVICSR:N WILLIAM PROF UATHEMATICS DEPT $P C$ BX 50

[U $Y$ E [ I

NEN LEALAND

LAVIES ROUNEY D PROF

NUFFIELC RADIO

JCCR-LL CANK

NACCLSSFIELD SK119DL

U.K.

EAVIS LEVERETT JR PRDF

$4.5-41$ CALTECH

PASADENA CA 91125

U. S.A.

CAVIS MOREIS S PROF

UEFT PHYS ASTRCNOMY

284 PHILLIPS H $39 A$

CRA PHILLIPS HE 2759

U.S. $\Delta$.

[AWE JEHN $\triangle L A N$ CR

DEPT OF ASTRONOMY

THE UNIVERS ITY

LEICESTER LEI TRH

U.K.

DE PGER K S OR

ASCHUURN USSERVATORY

475 A CHARTER STREET

MATISDN NI 53706

U.S.A.

CF FELICE FERNANEO CR CALILEI

UNIVERSITA DI PACOVA

ITALY 35 OS PAODVA

DE GP $\triangle A F F W$ DR STEREKUNOIG INST ZCANCNOURG 2

THE NETHERLANDS OE GRCCT MART CP ARNAGH BTGI 900 U.K.
DANESE LUIGI DR

OSSERVA TORIO ASTRO-

NOMICO

I-351CO PADOVA

CATLOWE AYTON DR

LOCKHEED P A RES LAB

DEPT $52-12$ BLDG 202

PALO ALTO CA 94304

U.S.A.

DAVIDSEN ARTHUR FALNES DR

DEPT OF PHYSICS

JOHNS HOPKINS UNIV

CHARLES AND 34 TH STS

U.S. IM.

DAVIES JOHN G DR

NRAL

JOURELL BANK

MACCLESFIELD

U.K.

DAVIS CEECIL G JR

UNIVERSITY OF

CALIFOKNIA LASL

LOS ALAMOS NM 87545

U.S.A.

DAVIS MARC DR

DEPT OF ASTRONOMY

HARVARD UNIVERSITY

6O GARDEN ST

CAMBR IDGE MA 02138

U.S.A.

DAVIS RICHARD J DR

NUFFIELD RAD IO

A STRONOMY LABS

JODRELL BANK

MACCLESFIELD SKI190L

U.K.

DE BERGH C DR

GROUPE PLANETES

SECT D.ASTROPHYSIQUE

OBS DE MEUDON

F- 92190 MEUDON

FRANCE

DE BRUYN ANTONIUS G DR

RÁDIOSTERREWACHT

POSTBUS 2

7990 AA DWINGE LOO

DE FREITAS PACHECOJ A DR

DBSERVATORRIO DE SAO

CAIXA POSTAL 30627

SAO PAULC

BRAZIL

DE GRAAUW TH DR

SPACE SC IENCE DEPT

ESA

NOORDWIJK

THE NETHERLANDS

DE GROOT T DR

STERR EKUNDIG

INST I TUUT

ZONNENBURG 2

$3 E 12$ NL UTRECHT
DANKS ANTHONY C R

E SO

SCHLEISSHEIMER ST 17

D-8046 GARCHING B M

GERMANY, F.R.

DAUBE-KURZEMNIECE I A DR

RADI OASTROPHYSICAL

ORSERVATORY LATVIAN

ACADEMY OF SCIENCES

226026 RIGA

U.S.S.R.

DAVIOSON KRIS DR

SCHOOL PHYS AND ASTR

UNI $V$ OF MINNESOT A

MINNEAPOLIS MN 55455 U. S.A.

DAVIES MERTON E MR

1414 SAN REMO ORIVE

PAC IF IC PALISADES

CA 90272

U.S.A.

DAVIS JOHN DR

SCHOOL OF PHYSICS

UNIVERSITY OF SYDNEY

SYDNEY NSW 2006

AUSTRALIA

DAVIS MICHAEL M DR

ARECIRO OBSERVATORY

$P$ O BOX 995

ARECIBO PR DO6I2

U.S.A.

DAVIS ROBERT J DR

CENTER FOR ASTRO

PHYSICS

GO GARDEN ST

CAMBRIDGE MA 02138

U.S.A.

DE BIASE G A DR

OSS ASTRONOMICO

VIALE DEL PARC?

MELLINI 84

ROMA

DE CASTRD A DR

OBSERVATORIO

ASTRONOMICO

ALFONSO XII 5

SPAIN

DE GRAAF T DR

INST VDOR FONET ISCHE

WE TENSCHAPPEN

GROTE ROZENSTRAAT 31

9712 TG GRONINGEN

THE NETHERLANDS

DE GREVE JEAN PIERRE DR ASTROPHYSICAL INST

$\checkmark$ U PLEINLAAN 2

BELGIUM BRUSSELS

DE JAGER CORNELIS PRDF

THE ASTRONOMICAL

INSTI TUTE

BENELUXLAAN 21

3527 HS UTRECHT 
DEPTAGER G OPR RFCEES UNIVERS ITY

GRAHAMSTOWN 6140

SCUTH AFRICA

CEKKOZT JULES J CR

G50 GV NIJMEGEN

THE NETHERLANCS

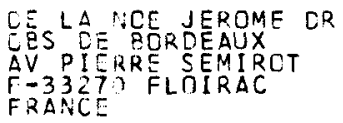

DE NARCUS WENOELL C PROF

KENTUCKY-LEXINGTON

LEXINGTCN KY 40506

U.S.A.

DE SABBATA $V$ PRCF OR

IST LI FISICA

UAIV DI BOLOGNA

IA IRIVERIO 46

ITALY

CE YCUNG DAVIC S CR

ECGENUNT RD

CFARLOTTESVILLE

VA $23 \Rightarrow$ l

CEFARHAT SUZANNE $V$ DR

CES DE PARIS

GI AV L'OBSERVATOIRE

FRAIVE 14 PARIS

CECAUX BERNARC
FORUE BOIS LE VENT
F-75.TIE PARIS FRARCE

CEHARVCNG JEAN MICHEL OR LAS TRAVERSE CU SIP-

HCA LES TROIS LUCS

FRANC:

DEKKER E $Q R$

STEREEWACHT

FCSTrius 3513

THE NETHERLANCS

DELECULLE LUC PAOF

ROPHYSICUE

UAIVERSITE UE LIEGE

ULGIJN

LELSFMME ARMAIC H PROF DR

L. TST OF PHYYSICS AND

ASTRC UCVYY

UAIVERSITY OF TOLECO

TCLEEC OH 436J6

U.
DE JONG T DR

ASTRONOMICAL INST

ROETER STRAAT 15

AMSTERDAM 1004

THE NETHERLANDS

CE KORTE P A J DR

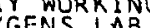

WAS SENAAR SEWEG 78

2300 RA LEIDEN

THE NETHERLANDS

DE LA REZA RAMIRO DR

OESERVATORID

NAC IONAL

R GEN BRUCE 586 ZCO8

2COOO RIO DE JANEIRO BRAZIL

DE MOTTONI Y PALACIOS DR

PRIVAT DBSERVATORY

I-16I45 GENOVA

ITALY

DE VAUCOULEURS G PROF

DEPT OF ASTRONOMY

UNIVERSITY OF TEXAS

AUSTIN TX 78712

U.S.A.

DE ZOTTI GIANFRANCO DR

OSSERVATURIO ASTRO-

NOMICO

$I=35100$ PADOVA

DEBEHOGNE HENR I DR SC

DE BELGIOUE

3 AVE CIRCULAIRE

B-1 180 BRUXELLES

DEEMING T J DR

DEPT OF ASTRONUMY

404 PHYSICS BLDG

UNIVERSITY OF TEXAS

AUSTIN TX 78712

U.S.A.

DEINZER W PROF OR

UTIVERSI TAETS-

STERNWAR TE

GE ISMARLANDSTR 11

D- 3400 GOETTINGEN

GERMANY, F.R .

DELACHE PHILIPPE J DR

OBSSERVATOIRE DE NICE

AP 252

F-O6OO 7 NICE CEDEX

FRANCE

DELCRO IX ANORE J S DR

$19 A$ E VANDER VELDE

$B-7230$ FRAMERIES
BELCIUM

DEMARCQ JEAN L ING OESERVATTOIRE DE NICE $B P 252$

F-06007 NICE CEDEX

FRANCE
DE JONGE J K DR

DEPT OF ASIRONOMY

UNIV OF PITTSBURGH

RI VERVIEW PARK

PITTSBURGH PA 15214

U.S.A.

DE LA HERRAN $\checkmark$ JOSE ENG

INSTITUTO DE

A STRONOMIA MEXICO

AP POSTAL 971

MEXICO 1 DF

DE LOORE CAMIEL PROF

ASTROPHYSICAL I NST

VRI JE UNIVERSITEIT

VAN BRUSSEL

BEIOSO BRUSSELS

DE PASCUAL MARTINEZ M DR

OBSERVATORID

ASTRONOMICD

ALFONSO XII 5

MAORID 7

SPAIN

DE VEGT CHR PROF DR

HAMBURGER STERNWARTE

GDJENBERGSWEG 112

$D-2550$ HAMBUR

DEARBORN DAVIDS P DR

STEWARD OBS

UNI Y OF ARIZONA

TUC SON AZ 85721

U.S.A.

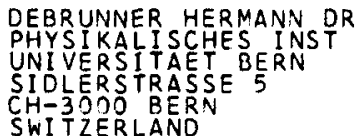

SWI TZERLAND

DEERENBERG AD J DR

COSMIC RAY WORKING

GROUP HUYGENS LAB

WASSENAARSEWEG 78

2300 RA LEIDEN

THE NETHERLANDS

DEJAIFFE RENE J DR

DBSERVATOIRE ROYAL

DE BELGIQUE

AVE CIRCULAIRE 3

BELGIUM

DELANNOY JEAN DR

OBSERVATOIRE DE

BORDEAUX

AVE PR P SEMIROT

F-33270 FLOIRAC

FRANCE

DELHAYE JEAN PROF

GI A VE DE

L OBSERVATOIRE

FRANCEE

DEMARET JACQUES DR

I58\%033 AVENUE DE

L DE SERVATOIRE

B-4 TOO LIEGE

BELGIUM 
DENARQUE P PROF

YALE UNIVERS ITY OBS

YALE STATION

NEW HAVEN CT 06520

U.S.A.

SEENIN V G PROF DR

TRCNGMICAL INSTITUTE

117234 MOSCOW

U.S.S.R.

DENIISYUK EDVARD K DR

ASTRUPHYSICAL INST

48,068 ALMA AT A

U.S.S.R.

\section{DENNISCN P A DR \\ UEPT CLF PHYSICS \\ ADELAIDE SA $50 O I$
ALSTRALIA}

DERMENDJIEV VLADIMIR DR DEPT OF ASTRONOMY

EULGARIAN ACAD OF SC

7 TH NOVEMEER STR 1

SCFI A

BULGAiRIA

CEUGNER FRANL-LUOWIG OR

INST F ASTRONOMIE U

AN HUBLANO

D-8700 WUERZBURC

GERNANY, F.R.

UEUTSCHMAN W.A. DR

KCSE-HULMAN INST.

5530 WAEASH AVE.

TERRE HAQASH AVE 87803

U.S.A.

DEWITT B S DR

UAIV OF TEXAS

AUSTIIV TX 78712

U.S.A.

CEZSC LORANT PROF

HEL IUPHYS I CAL

CESERVATORY

$H-4013$ DEBRECEN

HUNGARY

DIEAY E A DR

STERNBERG STATE AS-

117234 MOSCOW

U.S.S.R.

CICKEL JOHN R ASSOC PROF

UAIVERSITY OR

ILLINOIS OBSERVATORY

UREAMA IL 6 IBOI

U.S.A.

DIECKVOSS WILHELM KE PROF HANBURGER STERNWARTE [- $205 \%$ HAMBURG 85

GERMAQY, F.R.
DEMENKO A A DR

KIEV STA TE UNI VERSI-

SERVATORY

$252053 \mathrm{KIEV}$

U.S.S.R.

DENIS CARLO DR

INST D ASTROPHYSIQUE

UN IVER SITE OE LIEGE

A-42OO COINTE-OUGREE

BELGIUM

DENNIS RR IAN ROY DR

NASA-GSFC

CODE 684

GREENBELT MD 20771

U.S.A.

DENT WILLIAM A PROF

PFYSICS ASTRONOMY

DEPT GRC TOWER B

UN IVER SITY OF MASS

U.S.A.

DESESQUELLES JEAN DR

UN I VER SI TE LYON I

CAMPUS LA DOUA

F-69621 VILLEURBANNE

FR ANCE

DEUPREE ROBERT G DR

ROSTON UNIV

725 COMMONWEALTH AVE

U.S.S.A.

DEVINNEY E J DR

100 UNION AVE

DELANCD NJ 08075

U.S.A.

DEWITT JOHN H JR

3602 HOOOS HILLS RD

NASHVILLE TN 37215

U.S.A.

DI SEREGO A SPERELLO DR IST DI ASTRONOMIA

UNIV DI PADOVA

$I=35100$ PACOVA

DICKE ROBERT H PROF

JOSEPH HENRY LABS

PHYSICS DEPT

PR INCETON UNIVER SI TY

PRINCETDN NJ O8540

U.S.A.

DICKENS ROBERT J DR

ROYAL GREENWICH

OBSER VATORY

HAILSHAM

EAST SUSSEX BN27 IRP

U.K.

DIERCKSEN GEERD H F PH D MP I FUER PHYSIK UND ASTROPHYSIK

FOEHR INGER RING 6

D-8000 MUENCHEN 40

GERMANY, F.R.
DEMERS SERGE DR

DEPT DE PHYSIQUE

UNIV DE MONTREAL

CP 6128 SUCC A

$\begin{array}{lllll}\text { MONTREAL PQ H3C } & 3 \mathrm{~J} 7\end{array}$

CANADA

DENISSE JEAN F PROF

OBSERVATOIRE

DE MEUDON

$F=92190$ MEUDON

FRANCE

DENNISON E W DR

ELECTRONIC VISION CO 11526 SORRENTO

VALLEY ROAD

SAN DIEGO CA 92121

DEPRIT ANDRE PROF

CTR FOR APPLIED MATH

NATIONAL BUREAU OF

STANDARDS

WASHINGTON DC 20234

U.S.A.

DESPAIN KEITH HOWARD DR

DEPT OF ASTRONOMY

HAVERFORD COLLEGE

HAVERFORD PA 19041

U. S.A.

DEUTSCH ALEKSANDR N

PULKOVO OBSERVATORY

196140 LENINGRAD

U.S.S.R.

DEWHIRST DAVYD W DR

INST OF ASTRONOMY

THE OBSERVATORIES

MADINGLEY RD

CAMBRIDGE CB3 OHA

U.K.

DEWITT-MORETTE C PROF UNIVERSITY OF TEXAS AUSTIN TX 78712

U.S.A.

DI TULLIO GRAZIELLA DR OSSERVATORIO ASTRONOMICO

$1=35100$ PADOVA

DICKEL HELENE R OR

ILLINOIS OBSERVATORY

URBANA IL 61801

U.S.A.

DICKINSON DALE F DR

JPL 3 - 301

480 OAK GROVE DRIVE

PASADENA CA 91103

U.S.A.

DIETER NH DR

RADIO ASTRONDMY LAB

UNIV OF CALIFORNIA

BERKELEY CA 94720

U. S.A. 


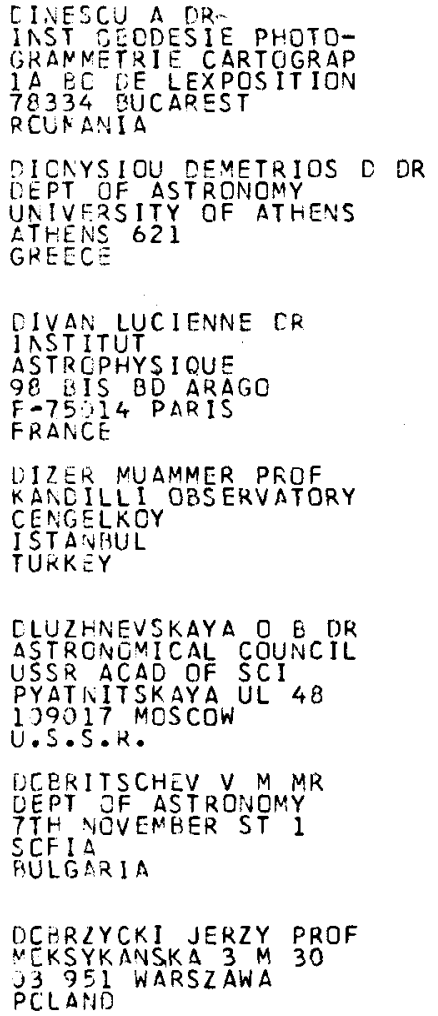

CLUZHNEVSKAYA O B DR ASTRONGMICAL COUNCIL PYATNITSKAYA UL 48 109017 MOSCOW U.S.S.K.

DCERITSCHEV Y M MR DEPT OF ASTRONOMY SCFIA IARIA

DCERLYCKI JERZY PROF MEKSYKANSKA 3 M 30 PCLAND WARSZAWA

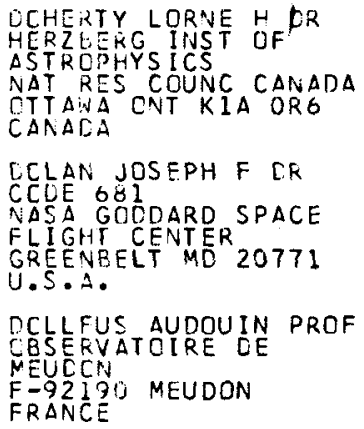

DCNKE HELMUT PH D TROPHYS RESA-LUXEMBURGST ITA CER-15. 2 POTSDAM G.C.R.

DCPITA MICHAEL A CR MT STRCMLO CESERVATORY PRIVATE B,AG 2600 AUSTRALIA

\section{CINGENS $P$ PROF DR STERRENKUNDIG INST KORTRIJKSE STWEG 523 $B-9000$ GENT \\ BELGIUM}

DIRIKIS MA OR

LATVIAN STATE UNI VER

SITY ASTRONOMICAL

OBSER VATORY

226098 RIGA

$U \cdot S \cdot S \cdot R$.

DIVARI $N$ E DR

ODESSA POLYTECHNICAL

INSTITUTE

270044 DDESSA

U.S.S.R.

DJURKOVIC PETAR M PROF

OBSERVATOIRE

DE BELGRADE

VOLGINA 7 G 11050 BELGRADE

YUGOSLAVIA

DOAN H H DR

OPSER VATOIRE DE LYON

F-69230 SAINT-GENIS

LAVAL

FRANCE

DOBRONRAVIN PETER P DR

CRIMEAN ASTROPHYSI -

CAL OBSERVATORY

334413 NAUCHNYJ

CRIMEA

U.S.S.R.

DODD RICHARD J DR

ROYAL OBSERVATORY

BLACKFURD HILL

EDINBURGH EHg $3 \mathrm{HJ}$

U.K.

\section{DOHERTY LOWELL R PROF UN IVER I IY WI SCONSIN \\ 475 N CHAR TER ST \\ MADISON WI 53706 \\ U.S.A.}

OOLGINOV ARKADY Z PROF DR IOFFE PHYSICAL

TECHN ICAL INSTI TUTE

194021 LENINGRAD

U.S.S.R.

DOMINKO FRAN PROF DR

SARANOVICEVA 11

61000 LJUBL JANA

YUGOSLAVIA

DOMMANGET J DR

OBSERVATOIRE ROYAL

DE BELGIQUE

AVE CIRCULA IRE 3

B-1 180 BRUXELLES

DORMANO JOHN R DR

MATHEMATICS OEPT

TEESSIDE POLYTECHNIC

MIDDLESBROUGH

CLEVELAND TSI 3BA

$U \cdot K$.
DINULESCU NICQLAE I PROF SOSEANA KISELEFF 13 72168 BUCURESTI RECTOR I

DISNEY MICHAEL J DR

OBSERVATORY

HAI L SHAM

EAST SUSSEX BN27 IRP

U.K.

DIXON ROBERTS S DR

OHIO STATE UNIVER-

SITY RADIO DBS

2015 NEIL AVE

COLUMBUS OH 43210

U.S.A.

DJUROVIC DRAGUT IN M DR

DEPT OF ASTRONOMY

FAC OF SCI ENCES

STUOENTSKI TRG 16

YUGOSLAVIA

DOAZAN VERA DR

DBSERVATOI RE

DE PARIS

GI AVE L OBSERVATOIR

F-750I 4 PARIS

DOBROVOLSKY OLEG V PROF

INST OF ASTROPHYSICS

734670 DUSHANBE

U.S.S.R.

DOGAN NADIR PRUF

A U FEN FAKULTESI

ANKARA

TURKEY

OOKUCHAEVA $Q D$ DR STERNBERG STATE ASTRONOMI CAL IN NS

U.S.S.R.

DOLIDZE M V DR

ABASTUMANI ASIROPHY-

SICAL OBSERVATORY

383762 ABASTUMANI

GEORG I A

U.S.S.R.

DOMINSKI IRENEUSZ DR

BOROWIEC

63-120 KDRNIK

POLAND

DONN BERTRAM D

GODDARO SPACE FLIGHT

CENTER

CODE 691

GREENBELT MD 20771

U.S.A.

DOROSHKEVICH A G DR

MATHE MATICS

USSR ACADEMY OF SCI

125047 MOS

U.S.S.R. 


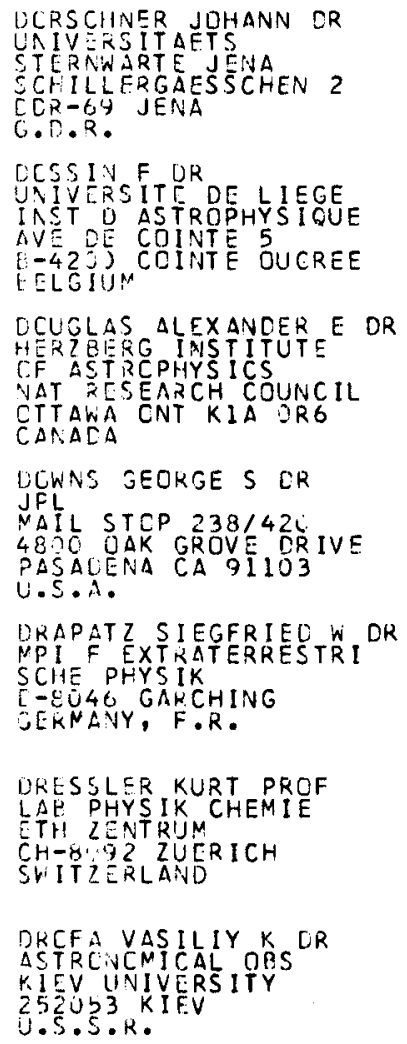

DUEIASKIJ B A DR INSTITUTE OF RACIO $\triangle C A D E M Y$ OF SCIENCES 1039.7 MOSCOW U.S.S.R.

CUCHESINE MAURICE DK CISERVATUIRE DOE PARIS EI AVE OESERVATOIRE $F=75$ I 4 PARIS FRANCE

DUFAY MAURICE PROF UAIVERSITE CLAUCE 43 ERARC-LYON I I I EMERE $F-63621$ VILLEURBANNE FRANCE

\section{DUFTCU PHILIP L CR QEPT OF APPLIED MATH BELFAST BT7 INN U.K.}

DUNA DMITRIJP DR NAIN ASTRONOMICAL OB $252127 \mathrm{KIEV}$

U.S.S.R.
DUS REIS M PROF

DIRECTOR OF THE

OBSERVATORY

COIMBRA

DOUGHTY NOEL A DR

PFYSICS OEPT

UNIVER SITY

CHR ISTCHURCH 1

NEW ZEALAND

DOUGLAS JAMES $N$ PROF

DEPT OF ASTRONOMY

UNIV OF TEXAS

AUSTIN TX 78712

U.S.A.

DRAKE FRANK D PROF ${ }_{402}^{N}$ SPÁCE SCI BLDG CORNELL UNIVERSITY

ITHACA NY 14853

U.S.A.

DRAVINS DAINIS DR

LUPD DBSERVATORY

SVANEGATAN 9

$S-222,24$ LUND

SWEDEN

DREVER RONALD W P DR DEPT OF NATURAL PHIL GLASGOW UN IVERSITY GLASGDW GI2 8QQ U.K.

DRYER MURRAY DR

SPACE ENVIRONMENT

$L A B$

NDAA ERL

BOULDER CO 80302

U.S.A.

DUBOSHIN G N PROF DR STERNBERG STATE ASTRONOM ICAL INS

U.S.S.R.

DUERBECK HILMAR W DR OBS HOHER LIST

UNIV STERNWARTE BONN

D- 5568 DAUN

GERMANY, F.R.

DUFLOT MARCELLE DR

MAR SE ILLE

2 PLACE LE VERRIER F-13004 MARSEILLE FRANCE

DULEY WALTER W PROF

PHYSICS DEPT

YORK UNIVERSITY DOWNSVIEW ONT M3JIP3 CANADA

DUMONT RENE DR

OBSER VATOIRE

DE BDRDEAUX

F -33270 FLOIRAC

FRANCE
DOSCHEK GEORGE A DR

NAVAL RESEARCH

LABORATORY

CODE 7125.8

WASHING TON DC 20375

U.S.A.

DOUGLAS A VIBERT OR

40267 SYDENHAM ST

KINGSTON ONT K7L $3 \mathrm{H} 2$

CANADA

DOWNES DENNIS D DR

MPI F RAOID-

ASTRONOMIE

AUF DEM HUEGEL 69

D-5300 BONN

DRAMBA $C$ PROF

DBS ASTRONOMIQUE

5 CUTITUL DE ARGINT

P.O.BOX 28

75212 BUCAREST

ROUMANIA

DRAVSKIKH A F DR

SPECIAL ASTROPHYSI-

LENI NGRAD BRANCH

196140 LENINGRAD

U.S.S.R.

DRILLING JOHN S

DEPT OF PHYSICS AND

ASTRONOMY

LOUI SI ANA STATE UNIV

BATON ROUGE LA TOBO3

U.S.A.

DUBAU J DR

OBS DE MEUDON

F-92I90 MEUDON

DUBOV EMIL E PROF

WDC -8

MOLODEZZHNAYA 3

117296 MOSCOW

U.S.S.R.

DUERST JOHANNES DR

SWISS FEDERAL OBS

SCHMELZBERGSTR 25

$\mathrm{CH} 8006$ ZUERICH

SWI TZERLAND

DUFLOT RENEE DR

OBSERVATOIRE

DE MARSEILLE

F-13004 MARSEILLE

FRANCE

DULK GEORGE A PROF

DEPT ASTRO-GEOPHYS

UNIV OF COLORADO

BOULDER CD 80302

U.S.A.

DUMONT SIMONE OR

INST D' ASTROPHYS I QUE 98 BIS BLVO ARAGO F-75014 PARIS 
DUNCAN ROBERT A PROF CSIRL

DIV CF RAOIOPHYS ICS

$P$ CE BOX 76

EFPING NSW 2121

AUSTRALIA

CUNHAM THEDDORE JR DR CHECCRUA NH O3817

U.S. $\triangle$.

LUPREE ANUREA K OR GU GARDEN ST

CANGRIDGE MA 02138

U.S. $A$.

DURNEY BERNARD R ER NAT ICHAL CTR FOR ATNCSPHER IC RES

BCULCER CO 80303

U.S.A.

CVCRAK RUDOLF DR ASTRCNGMISCHES INST UER UVIVERS ITAET

$A-8110$ GRAZ

AUSTRIA

DYER CHARLES CHESTER DR

PHYS SCS GR RM R L2Z

SCARLORCUGH COLLEGE

UAIV OF TORONTO

TCRONTO ONT MIG 357

CARAEA

CYSOV JOHN E CR

UN IVERSITY OF

NANCHESTER

NANCHESTER M13 PPL

U.K.

CZIGVASHVILI R M CR

AEASTUMAN ASTROPHY -

SICAL CBSERVATORY

$U . S . S \cdot R$.

ECCLES MICHAEL J CR INTUROH I

U.K.

ELNCNDS FRANK N JR CR

ULN IV 15212 STY OF TEXAS

AUSTIV TX 78712

U.S.A.

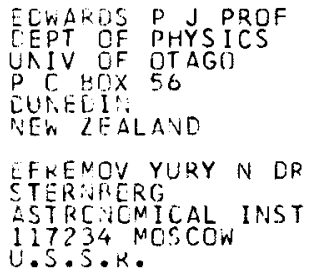

DEPT OF AEROSPACE

ENG INEER ING

UN IVERSI TY OF TEXAS

AUSTIN TX 78712

U.S.A.

DUNKELMAN LAWRENCE

CODE 685 LAB F ASTRO

AND SOLAR PHYSICS

GODDARD SPACE FL CTR

GREENB

DUPUY DAVID L DR

DEPT OF ASTRONOMY ST MARY S UNIVERSITY HAL IFAX NS B 3 H $3 \mathrm{C}_{3}$

CANADA

DURRANT CHRISTOPHER J DR

KIEPENHEUER INST

SCHOENECK STR 6

D-780O FRE IBURG

GERMANY, F.R.

DWORETSKY MICHAEL M DR

DEPT OF PHYSICS AND

ASTRONOMY

UN IV COLLEGE LONDON

LONDON

DYER EDWARD R OR

NATIONAL ACADEMY OF

SCIENCES

$215 I$ CONSTITUTION AV

WASHINGTON DC 20418

U.S.A.

DZHAP IASHVILI $V P$ DR

AEASTUMAN I A STROPHY-

SICAL OBSERVATORY

383762 ABASTUMANI

GEQR G I A

U.S.S.R.

EATON JOEL A DR

PENNA STATE UNIV

UNIV PK PA 16802

U.S.A.

EOOY JOHN A OR

OBSERVA TORY

$80 \times 3000$

BOULLER CO 80307

U.S.A.

EDMONDSON FRANK $K$ PROF

GOETHE LINK OBS

$319 A$ SWA IN HALL WEST

INDIANA UNIVER SITY
BLOOMING ION IN 47405

S.A.

EEL SALU HEINO DR

TOR -444 TURAVERE

ESTONIA

U.S.S.R.

EGGLETON PETER P DR

INSTITUTE DF

ASTRONOMY

MADINGLEY RD

CAMBRIDGE CB3 OHE

U.K.
DUNHAM DAVID W
COMPUTER SCS CORP
COLLESVILLE RD

SILVER SPG MD 20910

U.S. A.

DUNN RICHARD B DR

SACRAMENTO PEAK

OB SERVATORY

SUNSPOT NM 88349

U.S.A.

DURISEN RICHARD H DR

DEPT OF ASTRONDMY

SWA IN WEST 319

INDI ANA UNI V

BLOOMINGTON IN 4740 I

U. S.A.

DUTHIE JOSEPH G PROF

UNI VERSI TY ROCHESTER

DEPT OF PHYSICS AND

ASTRONDMY

ROCHESTER NY 14627

U.S.A.

DYCK M DR

KITT PEAK NATIQNAL

OB SERVATORY

$P$ O BOX 26732

TUCSON AZ 85726

U.S.A.

OYSON F J OR

ADVANCED $S$ TUDIES

PRINCETON NJ O8540

U.S.A.

DZIEMBOWSKI W DR ASTRONOMICAL CENTER

UL BARTYCKA 18

OO-716

EBBIGHAUSEN E G

PHYSICS OEPT

EUGENE OR 97403

U. S.A.

EDLEN BENGT PRQF

DEPT OF PHYSICS

SDEL VEGATAN 14

$5-223$ 62 LUND

EDWARDS ALAN CH DR

DEPT OF ASTROPHYSICS

SOUTH PARKS ROAD

OXFORD OXI $3 R Q$

U.K.

EFREMOV YU I OR

MATHEMATICS

USSR ACADEMY OF SCI

125047 MOSCDW

U.S.S.R.

EHLERS JURGEN PROF

MPI FUER PHYSIK UND A STROPHYSIK MUENCHEN D-8000 MUENCHEN 40

GERMANY, F.R. 


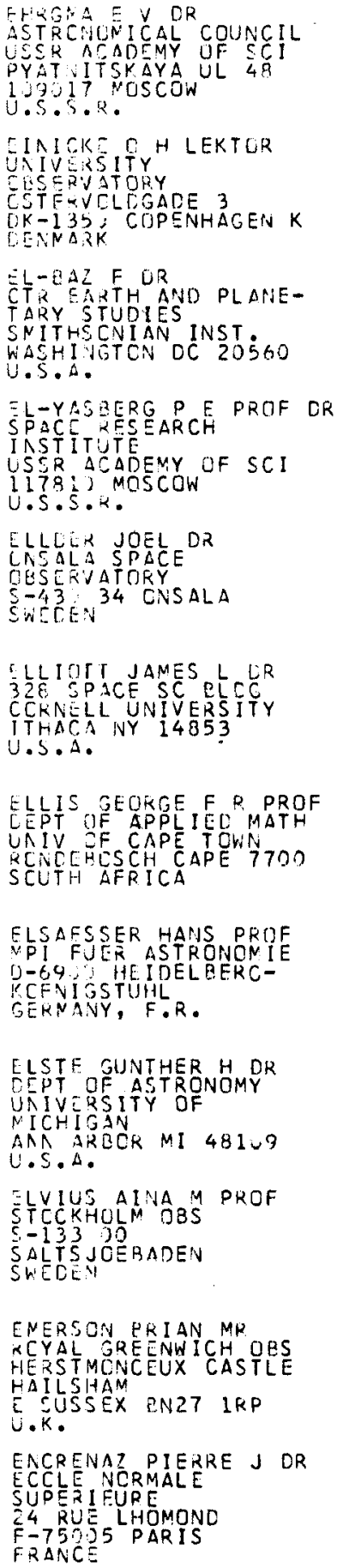

ELSTE GUNTHER H DR CEPT OF ASTRONOMY UAIVERSITY OF NICHIGAN

EICHHORN HEIIJRICH K OR DEPT OF ASTRONOMY

UN IV OF FLOR IDA

231 SPACE SC RES $B L D$

GAINESVILLE FL 32611

U.S.A.

EINSATO JAAN DR

$2 O 2444$ TORAVERE

ESTONIA

EL-RAEY MOHAMED E DR

DEPT OF PHYSICS

FACULTY OF SCIENCE

ALEXANDRIA EXANORIA

ELFORD W $O$ DR

DEPT OF PHYSICS

UNIV OF ADELAIDE

ADELAIDE

ELLIOT HARRY PROF BLACKETT LABORATORY IMPER IAL COLLEGE

LUNDDN SW7 2EZ

U.K.

ELLIOTT KENNETH H DR

DEPT OF SPACE RES

UNIV OF BIRMINGHAM

$P 0 \mathrm{BOX} 363$

BIKMINGHAM BIS 2 TT

U.K.

ELLYETT CLIFTON D PROF DEPT OF PHYSICS UNIVERSITY NEWCASTLE AUSTRALIA ELSMORE BRUCE DR MADINGLEY ROAD CAMBR IDGE CB 3 OHE U.K.

ELSTON WULFGANG E PROF DEPT OF GEOLOGY

UNIVERSITY

ALBUQUERQUE NM 87131

U.S.A.

ELVIUS TORD PROF

LUND OESERVATORY

SVANEGATAN 9

$S-22224$ LUND

SWEDEN

EMERSON JAMES $P \quad R$

DEPT OF PHYSICS

MILE END ROAD

LONDON

ENCRENAZ T DR

GROUPE PLANETES

SECT D'ASTROPHYSIQUE QBS DE MEUDON F-92190 MEUDON
EICHLER DAVID DR

ASTRONOMY PRDGRAM

UNIV OF MARYLAND

COLLEGE PK MD 20742

U.S.A.

EKERS RONALD J DR KAPTEYN LABORATORIUM

POSTBUS 800

GRONINGEN

THE NETHERLANDS

EL-SHAARAWY M B DR

HELWAN OBSERVATORY

HELWAN NEAR CAIRO

ARAB REP. OF EGYPT

ELGAROY OYSTEIN PROF

INST THEOR ASTROPHYS

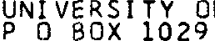

BLINDERN OSLO 3

\section{ELLIOTT IAN DR}

DUNSINK OBSERVATORY CASTLEKNOCK

CO DUBLIN

ELLIS G R A PROF

UNIV OF TASMAN

HOBART TASMANIA

AUSTRALIA

ELMEGREEN BRUCE GORDON DR DEPT OF ASTRONOMY

PUPIN LABS

COLUMBIA UNIY

NEW YORK NY 10027

U.S.A.

ELST E DR

KONINGLI JKE STERREN-

WACHT VAN BELGIE

RING LAAN 3

B-1IBO BRUSSEL

ELVIS MARTIN S DR 46 HOWARD ROAD LEICESTER

U.K.

ELWERT GERHARD PRQF TI SCHE ASTROPHYSIK UNIV TUEB INGEN D-7400 TUEBINGEN GERMANY, F.R.

EMINZADE T A DR SHEMAKHA ASTROPHYSI CAL OBSERVATORY 373243 AZERBAIDZAN U.S.S.R.

ENDAL ANDREW S DR DEPT PHYSICS ASTRON LOUISI ANA STATE UNIV U.S.A. 
ENGELHARD E J G PROF DR $S A C K$
$C-33$

CERNANY, F.R.

ENCME SHINZO ASSCC PROF RESEARCH INST I TUTE AAGCYA UNIVERSITY TCYCKAWA 442 JAPAN

EPSTEIN EUGENE E DR $130-422$ AERO CORP UCX 92357

LCS AIVELES CA 90009 U.S.A.

EPSTFIN RICHARD I CR NCP.DITA

PLEGCAMSVEJ 17

UK-213O COPENHAGEN?

CENMARK

ERIKSEN GUNNAR PRDF IAST THEOR ASTROPHYS UAIVERSITY OF OSLO

$P[10 \times 1329$

HLINUEKN OSLOO 3

NCRWAY

ERUSHEV A $N$ QR

CRIMEAV ASTRCJPHYSI-

CAL OBSERVATORY USSR

$\triangle C A D C F$ CSCI NAI

U. S.S.R.

ESKICULU A NIHAT

NALTEPE ANKARA

TURKEY

EVANS DAVID S PREF

UA IVERSITY OF TEXAS

AUSTIN TX 78712

$U . S . A$.

EVANS KENTON C CR

PHYSICS DEPT

THF UNIVERSITY

LEICESTER LEI TRN

L.K.

EVANS HOGER O CR

KUTHERFORD

LABCEATCRY

EICCT JX11 JaX

U.K.

EVIATAR AHAROW PRUF

AEFT CF COEPHYS ICS

TE-AVIV UNIVERS ITY

$T E L-A \cup[V$

$I S K A=t$

EER CILHAN PROF

ICDLE EAST ECEF-

ANKARA

TUHKEY
ENGIN SEMANUR ASSOC PROF DEPT OF ASTRONOMY

UNIVER SITY OF ANKARA

FEN FAKULTESI

ANKARA

TURKEY

ENSL IN HEINZ DR

DEUTSCHES HYDROGRA-

PHISCHES INSTITUT

POSTFACH 220

D- 2000 HAMBURC 4

EPSTEIN GABRIEL LEO OR

NASA-GSFC

CODE 682

GREENBELT MD 20771

U.S.A.

ERDI $\&$ DR

ASTRONOMICAL DEPT

LORAND EOTVOS UNIV

$K$ KUN BELA TER 2
$H-1083$ BUDAPEST

HUNGARY

ERPYLEV N P DR

A STRONOM I CAL COUNCIL

USSR ACADEMY OF SC

109017 MOSCOW

U.S.S.R.

ESHLEMAN VON $R$ PROF

DURAND 221

STANFORD UNI VER SITY

STANFORD CA 94305

U.S.A.

ESPOSITO F PAUL PROF

DEPT OF PHYSICS 11

UII IVER S I TY OF

CINCINNATI

C INCINNATI OH 45221

U.S.A.

EVANS J V DR

MIT L INCOLN LAB

$M$ ILLSTONE / HA YSTACK

DBSER VATORY

LEXINGTON MA 02173

U.S.A.

EVANS NANCY R DR

183 BR IAR HILL AVE

TORONTO ONT MAR IHE

CANADA

EVUOKIMOV YU $V$ OR

ENGELHARDT ASTRONOMI

CAL OASERVATORY

42252 E KALAN

U.S.S.R.

EWEN HARDLD I OR

WESTON MA OQRAO

U.S.A.

FABER SANDRA M PROF LICK UBSER VA TORY

UN IVER SITY OF

CAL I FOKN IA

SANTA CRUZ CA 95064

U.S.A.
ENGVOLD DDDBJOERN DR

UNIVERSITY OF OSIO

$P O B O X 1029$

BLINDERN OSLO 3 NORWAY

EPPS HARLAND WARREN PROF

DEPT OF ASTRONOMY

UNI VERS ITY OF CALIF

MATH SCI RM 8983

LOS ANGELES CA 90024

U.S.A.

EPSTEIN ISADORE PROF

ASTRONOMY DEPT

COLUMBIA UNIVERSITY

NEW YORK NY 10027

ERICKSON WILLIAM C OR

ASTRONOMY PROGRAM

UNIVERSITY MARYLAND

COLLEGE PARK

$M D 20742$

U.S.A.

ERSHKOVICH ALEXANDER DR $58 / 1$ HANKIN $5 T$

RAANANA

I SRAEL

ESI POV $V F$ DR

STERNAERG STATE AS-

TRONOMICAL INSTI TUTE

117234 MÓSCOW

U.S.S.R.

EVANGELIDIS E DR

UNIVERSITY OF PATRAS DEPT OF ASTRONOMY PATRAS

EVANS JOHN WDR

SACRAMENTO PEAK

OBSERVATORY

SUNSPOT NM 88349

U.S.A.

EVANS NEAL J II ASST PRDF

DEPT OF ASTRONOMY

AUSTIN TX 78712

U.S.A.

EVERHART EDGAR DR

PHYSICS DEPT AND

CHAMBERLIN OB DENVER

DENVER CO $8020 \mathrm{~A}$

U.S.A.

EWING MARTIN $S$

PALTECH 102-24 25

U.S.A.

FABIAN ANDREW C OR

INSTI TUTE

MACINGLEY ROAD

CAMBRIDGE CB3 OHE

U.K. 
FABRE HERVE DR

2 AVENUE MARECHAL

F-O6310 BEAULIEU MER

FRANCE

FAHR HANS JOERG PROF DR

INST ASTROPHY EXTRA-

AUF CEN HUEGEL 71

GERNAVY, F.R.

FALCIANI KOBERTO DR

CAPCOIMCNTE ASTRO

UPSERVATORY

VIA NOIARIELLO 16

I -8OI 3 I NAPOL

ITALY

FALLE SAMUEL A DR

UAIVEKSITY OF LEECS

LEEDS LS2 9JT

U. $k$.

\section{FANT I CARLA GIOVANN IN I \\ NIA DI RADIOASTRONO- \\ VIA IRNERIO 46 \\ I -41$) 126$ BOLOGNA \\ FARINELLA PAÕ்O DR \\ CSSERVATORIO ASTRO- \\ NONICO \\ $E R E R$ \\ II $L A N O$}

FAUEKVER DDNALD J DR

NT STRLMLO

WCOEN PO ACT 2606

AUSTRALIA

FAY THEODORE C CR

MAIL STOP I9

HUNTSVILLE AL 35607

U.S.A.

FEAUTRIER NICOLE CR

CESEPVITOIRE

DE NEUTCCM

$F-92130$ MEUDON

FRANCE

TECOROVA RIMMA T OR

$\triangle S T R C$ IOMICAL OBS
3270$,$) NIKOL AEV$

U.S.S.R.

FEIEELMAY WALTER A DR

NASA-SSFC

GREENBELT MD 20771

U.S.A.

FEITZINGER JOHANNES $V$ PHD ASTRCNCMISCHES INST

QUHR UNIVERS IT AET

PCSTFACH 102148

GERMANY, F.R.
FAELTHAMMAR CARL GUNNE PR DEPT PLASMA PHYSICS ROYAL INST OF TECHNOLOGY S-IDO 44 STOCKHOLMTO
SWEDEN

FAHY EOWARO F PROF PHYSICS DEPT

UNIVERSITY COLLEGE

CORK

IRELAND

FALK SYDNEY W JR DR

UNIV DF TEXAS

AUSTIN TX 78712

U.S.A.

FALLER JAMES E PROF JILA NBS TY COLORADO BOULDER CO BO3O9

U.S.A.

FANT I R DR

PFYSICAL INST

UNIV OF BOLOGNA

$V I A$
I IRNER IO 40126 BOLOGNA

ITALY 26 BOLOGNA

FATCHIKHIN NIKOLAJ V DR PULKOVO OBSERVATORY 196140 LENINGRAD

U.S.S.R.

FAULKNER JOHN PROF

UNIVERSITY OF

CAL IFORN IA

SANTA CRUZ CA 95064

U.S.A.

FAZIO GIOVANNI G DR CENTER FOR GO GARDEN ST CAMBR IDGE MA 02138

U.S.A.

FECHTIG HUGO DR

SANSER WEG

D- 9906 LE I MEN

GERMANY, F.R.

FEGAN DAVIDJ DR

PHYSICS DEPT

UNIV CDLLEGE

BELFIELD

IRELAND

FEINSTEIN A DR

1900 LA PLATA

ARGENTINA

FEIX GERHARD DR

RUHR UNIVERSITAET

CEPT XI I

POSTFACH $132 \quad 148$

D-4630 BDCHUM
FAHLMAN GREGORY G DR DEPT GEOPHYS AND AST UNI VERSITY OF B C 2075 WE SBROOK PLACE VANCOUVER BC VGT IW5 CANADA

FAIRALL ANTHONY P DR

DEPT OF ASTRONOMY

UNIV OF CAPE TOWN RONOEBOSCH 7700 CAPE

SOUTH AFRICA

FALL $S M D R$

INST OF ASTRONOMY

MADINGLEY ROAO

CAMBRIDGE CB3 OHA

U. K.

FALLON FREDERICKW DR

DEPT OF ASTRONOMY

UNIV OF S FLORIDA

TAMPA FL 33620

U. S.A.

FARAGGIANA ROSANNA PROF

OSSERVATORI O

I I T T I I I POLO TRIESTE

FAUCHER P DR

OBS DE NICE

B P 252

F-06007 NICE CEDEX

FRANCE

FAWELL DEREK R DR

UNIVERSITY OF LONDON

QBSERVATORY

MILL HILL PARK

LONDON NW7 205

U. K.

FEAST MICHAEL W DR

P A A BOX 9

OBSERVATORY

CAPE 7935

SOUTH AFRICA

FEDOROV E P DR

UKRAINI AN ACAD OF SC

GOLOSEEVO

252127 KIEV

U.S.S.R.

FEHRENBACH CHARLES PROF

OBSERVATOIRE DE

HAUTE PROVENCE

ST MICHEL L OBS

F-0430 FORCALQUIER

FRANCE

FEISSEL MARTINE

OBSERVATOIRE PARIS

61 A VE DE

L-OBSERVATOIRE

FRANCE

FEJES I TVAN DR

GOMI SATELLITE

PO BOX 546

1373 BUDAPEST 
FELDNAN PAUL A CR HERZEERG INST ASTROD 1.) SUSSEX OR

CT AWA CNT KIA ORG CANADA

FELEABCK PAUL DR $F-32195$ MEUDON

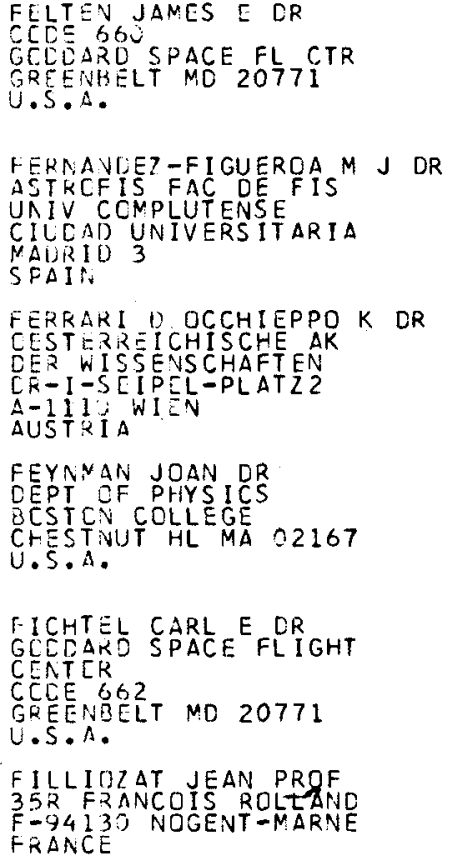

FINN $\ddot{C} C R$

INST TCR ASTRONOMY 2525 CCRREA ROAO

U.S.A.

FIRNANI CLAUDIO A PROF

INST ITUTO DE

ASTRCNOMIA UNAM

NEXICO $200^{\circ}$ DF

NEXICO

FISCHEL DAVID DR

CCDE 671 I

GKESNBELT MD 20771

U.S.A.

FISHER J RICHARE

NRAC

$P$ C BOX 2

GREEN BANK WV 24944

U.S.A.
FELOMAN PAUL DONALD DR JOHNS HOPKINS LNIV

BAL TIMORE MD 21218

U.S.A.

FELLGETT PETER 8 PROF CEPT OF CYBERNETICS 3 EARLY GATE WHITEKNIGHTS

READ ING RGE 2AL

U.K.

FENKART ROLF $P$ DR ASTRONOMI SCHES INST UN IVERSI TAET BASEL VENUSSTR 7 CH-4IO2 BINNINGEN SWITZERLAND

FERNIE J DONALO PROF

DAVIO OUNLAP DOS

$P$ O BOX 360

RICHMOND HILL

ONT L $4 C$ C 4 Y

CANADA

FERRAL-MELLO S PROF DR

UN IV DE SAO PAULO

DEPT ASTRONOMI

CAIXA POSTAL 30627

OIOOO-SAO PAULO SP

BRAZ IL

FIALA ALAN D DR

WASHANGTON DC 20390

U..S.A.

FIELD GEORGE 8 PROF

CENTER FOR

ASTROPHYSICS

CAMBRIDGE MA 02138

U.S.A.

FINDLAY JOHN W DR

NATRADIO ASTR OBS

EDGEMONT RD

CHAR LOTTESVILLE

$\checkmark A, 2290$

U.S.A.

FINZ I ARR IGO DR

DEPT OF MATHEMATICS

TECHNION I I T

HA IFA

ISRAEL

FIRNE IS MARIA G DR INST FUR ASTRONOMIE TUERKENSCHANZSTR 17 $A-1180$ WIEN

AUSTRIA

FISCHER P LEANDER PRDF FREYUNG 6 A-IDIO W

FISHER PHILIP C 2401 SHARON OAKS DR MENLO PARK CA 94025 U.S.A.
FELDMAN U DR DEPT OF PHYSICS AND ASTRONOMY

TEL-AVIV UNIV

RAMAT-AVIV

I SRAEL.

FELLI MARCELLO DR OSSERVATORIO ARCETRI LARGO FERMI 5 I TSDLY 25 FIRENZE

FENTON K 8 DR

DEPT OF PHYSICS UNIV QF TASMANIA AUSTRALIA

FERRARI ATTILIODR ISTITUTO DI FISICA CR M D AZEGLID 46 I -10125 TORINO

FESTOU M DR

SERVICE DIAERONOMIE CNRS B P NO 3 F-91370 VERRIERES LE BUI SSON

FICARRA A MR

LAB NAZI ONALE DI

RAOIOASTRONOM VIA IRNERIO 46

FIELDER GILBERT DR LUNAR AND PLANETARY UNIT E S DEPT VES TYY LANCASTER UNIVERS I TY

U.K.

FINK U DR

LUNAR AND PLANETARY LABORATORY UNIVERSITY ARIZONA

TUC SON AZ 85721

U.S.A.

FIREMAN EDWARD L

ASTROPHYSICAL OBS

60 GARDEN ST

CAMBRIDGE MA 02138 U.S.A.

FIROR JOHN W DR NATIONAL CTR FOR AT MOSPHERIC RESEARCH $P O B O X 3000$ BOULDER CO 80703 U.S.A.

FI SCHER STANISLAV DR ASTRONDMICAL INST CSAV

BUDECSKA 6

CZO 23 PRAHA 2

FI SHER RICHARD R DR

HIGH ALTI UODE OBS

$P$ BOX 3000

BOULDER CO 80307

U.S.A. 
FISHKOVA L M PROF $\triangle$ PASTUMANI ASTROPHYSICAL CBSERVATORY 38376.2 ABASTUMAN I GECRGIA

FIX JOHN U DR ASTRCNCMY

UNIVERSITY: OF IUWA

ICWA CITY IA 52240

L.S.A.

FLEISCHER ROBERT CR W 733 CHURCH ST N W (4) $A S H I N G$

FLQREVSKY KYRILLP DR

VERNADSKY INS

47 A VEROBJOVSKOJE

117334 MOSCOW

U.S.S.R.

FLCRSCH ALPHONSE CR

QESERVATOIRE

II RUE L UNIVERSITE

$F-67$ O) STRASBOURG

FRANC:

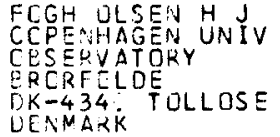

FONENKT ALEXANCER F CR

$\begin{array}{lll}\text { FCNEFKE ALEXANEER F CR } & \text { CR } \\ \text { SPECIAL ASTROPHYS OB }\end{array}$

MIZHNIJ ARKHYZ

$35714 J$ STAVROPOL SKIJ

KRAJ

U.S.S.R.

FCRBES J E DR

INCIANAPOLIS IN46208

U.S.A.

FCRNAN WILLIAM RICHARD DR

SNITHSCNI AN OES

COMPRIDEE MAROZI38

U.S.A.

FCRT 1 CIUSEPPE OK

CSSERVATORIO ARCETRI

LARGC FERMI 5

ITALY

FCSSAT ERIC DR

CESERV 252

F 67 NICE CECEX

FCX KENNETH DR

DEPT PHYS ICS ASTRON

UAIV OF TENNESSEE

NENPHIS TI 38133

U.S.A.
FITCH WAL TER S DR

STEWARD OB SER VATORY

UNIV OF ARIZONA

TUCSON AZ 85721

$U \cdot S \cdot A$.

FLANNERY BRIAN PAUL DR

GO GARDEN STREET

CAMBR IDGE MA 02138

U.S.A.

FLETCHER J MURRAY

OOM INION ASTRQPHYSI -

CAL OBSERVATORY

5071 W SAANICH RR7

$\checkmark$ ICTOR IA BC V $8 \times 3 \times 3$

CANADA

FLORENTIN-NIELSEN R M SC COPENHAGEN UNI VERSITY OBSER VATOR BRORFELDE

OK -4340 TOLLOSE

DENMARK

FLOWER DAVID R DR

DEPT OF PHYSICS

UNIVERSITY OF DURHAM

DURHAM DHI 3LE

U.K.

FUKKER AAD D DR

STERREWACHT

SONNENBORGH,

ZONNENBUR

THE NETHERLANOS

FONTAINE GILLES DR

DEPT OF PHYSICS

$P$ BOX 6128

MONTREAL PQ H3C $3 J 7$

CANADA

FORD HOLLAVD C ASSOC PRCF

DEPT OF ASTRONOMY

LOS ANGELES CA 90024

U.S.A.

FURT BERNARO $P$ DR

DBSER VATO IRE

DE MEUDON

$F-92190$ MEUDON

FRANCE

FORTINI TERESA DR

QSSERVATOR IO

ASTRONOMICO

I-00136 ROMA

ITALY

FOUKAL PETER $V$ DR

CENTER FOR

ASTROPHYSICS

6C) GARDEN ST

CAMBR IDGE MA 02138

U.S.A.

FOX $W$ E MR

4) WINDSOR ROAD

NEWARK NOTTINGHAMS

U.K.
FITZGERALD M PIM PROF

OEPT OF PHYSICS

UNI VERS ITY WATERLOD

WATERLOO DNT N2L $3 G 1$
CANADA

FLECKENSTEIN J D PROF OR

UNIVERSTTAET

RHE INSPRUNG 21

SWITZERLAND

FLIEGEL HENRY $F$

JPLO OAK GROVE DRIVE

PASADENA CA 91103

U.S.A.

FLORIDES PETROS S PROF

SCHODL OF MATHEMA-

DUBLIN 2

I RELAND

FOGARTY WILLIAM G DR

2511 PLOVER RD

WI SCONSIN RAPIDS

W. S.A.

FOMALONT EODWARD B DR

NATI ONAL RADID

ASTRONOMY

SOCORRO NM 87801

U.S.A.

FORBES ERIC G DR

HISTORY DEPT

EDINBURGH UNIVERS ITY

5O GEORGE SQUARE

EDINBURGH EH8 9 JY

U. K.

FORDWK JR DR

TERRESTRIAL MAGNET

CARNEGIE INSTITUTION

5241 BRDAD BR RD N W WASHINGTON OC $20015^{\circ}$

U. S. A.

FORT DAVID NORMAN DR 733 LONSDALE ROAD

OTTAWA KIK DJ9

CANADA

FOSBURY ROBERT A E DR TELESTCOPE PROJECT C 10 CERN

CH-12II GENEVA 23

SWI TZERLAND

FOWLER WILLIAM A PRCF

CALTECH 106 38.25

U.S.A.

FOY RENAUD DR

OBSERVATOIRE

DE MEUDON

F-92 I90 MEUDON 


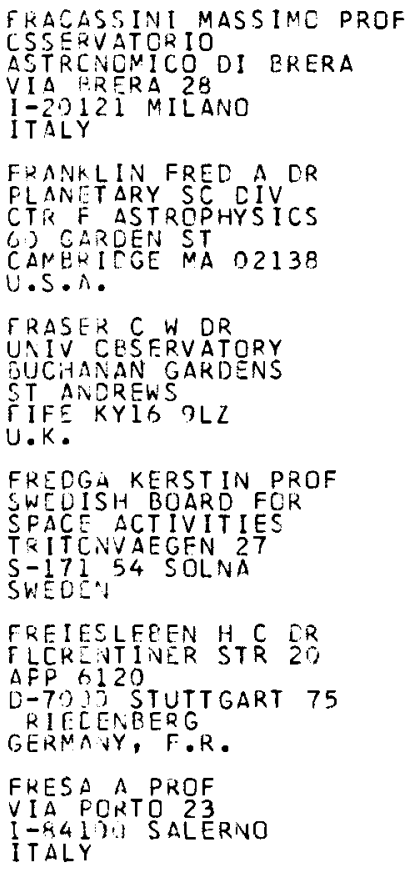

FKICKE WALTER PREF DR OIRECTOR ASTRONOMISCHES RECHENINST ITUT 12-14 NCENCHHOFSTR U-69: HEIDELBERG

GERNANY, F.R.

FKIELLANDER MICHAEL PROF CEPT UF PHYSICS W'ASHINGTON

UNIVERSITY

ST LCUIS MO 63130

U.S.A.

FRISCH HELENE DR CPSERVATOIRE DE NICE BP $2 \leq 2$

$\mathrm{F}-1,0,57$ NICE-CECEX

FRANCE

FRCESCHLE CHRIST IANE D DR CESERV F 673 NICE CEDEX FRANCE

FRCLCV M S DR

ASTRENOMICAL COUNCIL USSR ACADEMY OF SCI 1 UOD 17 MOSCOW

ij.S.S.R.

FUCHS JCSEPH PROF DR GELCSCHLAGSTR 185-9 $A-114$ WIEN

AUSTRIA
FRACASTORO MARIO G PROF OSSERVATORIO ASTRONO MICO DI TOR INO

P INO TOR INE SE

ITALY

FRANTSMAN YU L DR

RADIOASTRUPHYSICAL

OBSER VATORY LATVIAN

ACADEMY OF SCIENCES

226026 RIGA

U.S.S.R.

FRATER RQBERT HOR UN IVERSITY OF SYDNEY SYDNEY N S W 2006 AUSTRALIA

FREDR ICK LAURENCE W PROF EAANDER MCCORMICK OBSERVATORY BOX 3818 CHARLOTTESVILLE

VA 22903

U.S.A.

FREIRE FERRERO R G DR

DEPT ASTRDPHYS FOND

OBS DE PARIS

5 PLACE JANSEN

F-92190 MEUDON

FRANCE

FRESNEAU A DR

OBS DE STRASBOURG

11 R DE L UN IVER SITE FRANCE

FR IEDEMANN CHR ISTIAN DR

UN IVER SI TAETS

STERNWARTE JENA

SCHILLERGAESSCHEN 2

DDR - 69 JENA

$G \cdot D \cdot R$.

FR IEDMAN HERBERT DR US NAYAL RESEARCH LAB CODE 7100

WASHINGTON DC 20375

U.S.A.

FRISCH UR IEL DR OBSERVATOIRE DE NICE $B P 252$ F-06007 NICE-CEDEX

FROESCHLE CLAUDE DR $B$ P 252 F-O6007 NICE-CEDEX FRANCE

FROST KENNETH J DR

NASA GSFC

GREENBELT MD 20771

U.S.A.

FUJ IMOTO MASAYUK I DR NI IGATA UN I VER SITY 1-7-2 SAIJYO-MACHI JYOETSU

NIGATA 943

JAPAN
FRANDSEN SOEREN PROF INST OF ASTRONOMY UNI VERSI TY OF AARHUS OK- 8000 AARHUS $C$ DENMARK

FRANZ OTTO G DR LOWE LL OBSERVATORY $P$ O BOX 1269 FLAGSTAFF AZ 86002 U.S.A.

FRAZIER EDWARD N DR AEROSPAC CORPORATION $P$ BOX 92957 LOS ANGELES CA 90009 U.S.A.

FREEMAN KENNETH C DR MT STROMLO OBSERVATORY WODEN PO ACT 2606 AUSTRALIA

FREITAS MOURAO R R UR OBS NACI ONA

R GENERAL BRUUCE 586

SAO CRISTOVAO

RID DE JANEIRO BRAZIL

FRICKE KLAUS DR

UNI VERSITAETSSTERNW

UNI V GOE TT I NGEN GE I SMAR LANDSTR 11 D=340O GOETTINGEN GERMANY, F.R.

FRIEOJUNG MICHAEL DR INST D ASTROPHYSIQUE 98 I S BLVD ARAGO FRANCE

FRINGANT ANNE-MARIE DR

OBS DE PARIS

61 AV L'OBSERVATOIRE F-75014 PARIS

FRITZOVA-SVESTKA L OR 147 DOPPERSTRAAT

BUNSCHOTEN

THE NETHERLANDS

FROGEL JAY A DR CERRO TOLOLO INTERAMERIC OBSERVATORY CASILLA 63 D LA SERENA

FRYE GLENN M PROF PHYS DEPT ROCK RLDG CASE WE STERN RESERVE UNIVERSITY CLEVELAND OH 44106 U.S.A.

FUJIMOTO MITSUAKI DR DEPT OF PHYSICS NAGOYA UNI JAPAN 
FU JITA TAY YISHIO PROF UAIVERSITY OF TCKYO FUAKYD KU

ICKYC; 113

JAPAR

FUREILLIDK INGENAR DR

KITT DEAK NATICNAL

CESERVATORY

$P C$ Cox 26732

TUCSSCN AZ 85726

GASRIEL MAUR ICE R DR

IIST ASTRDPHYSIGUE

UNIVHSITE DE LIEGE

$\begin{array}{ll}E & -4 \\ E & 0\end{array}$

GAIL H P DR THERTAPYS

LER UNIVERSITAET

IN NEUENHEIMER F 294

1) -69 ) HEIDELEERG I

GERN $\triangle N Y, F, R$.

CALECTTI IERO PROF

CAPQRATORIOS DI

CCRSC FIUME 4

ITALY 33 TORINO

GALLAGHER III JOHN S DR CUSERVATORY

ITY DF

ILLINOIS

LREANA IL 61801

U.S. . .

\section{GALLCIET LOUIS CR \\ CSTERVATOIRE \\ E I AVE CBSERVATOIRE \\ $F-75314$ PAR IS \\ FRANCE}

GANALELOIN ABQULLA I DR

HELWAN CESERVATCKY

$\triangle R \triangle B$ REP. OF EGYPT

SAPCSHKIN SERGEI ILLAR DR
HARVARO COLLEGE

CESERVATORY

F. J GAROEN ST

CANPRIEGE MA D2138

U.S.A.

GARLICK GEORGE F CR

LET SCUTH B. BELOIT AVE

U.S.A.

GARINICK ROPERT ING

CESERVATOIRE DE LYON

F-69230 ST-GENIS

LAVAL

GARTCNW R S PROF

QLACKETT LABORATORY

INPERIAL COLLEGE

LCNCCN SW7 $28 Z$

U.K.
FUKUI TAKACJ DR

DEPT QF LIBERAL ARTS

DOKKYO UNI VERSITY

SAKAE-MACHI 600

SOKA SAITAMA

JAPAN

FUR SENKC M A DR

INSTI TUTE OF THEORE -

TICAL ASIRONOMY

192187 LENINGRAD

U.S.S.R.

GAHM GOESTA F DR

STOCKHOLM

OBSERVATORY

$S-13300$

SALTSJOEBADEN

GAIZAUSKAS VICTOR DR

HERZEERG INS

NAT RES COUNC CANADA

OTAWA ONT KIA ORG

AN ADA

GALIBINA I V DR

IICAL A STRONOMY

U.S.S.R.

GALLET R $M$

964 TTH STREET

BOULDER CO 80302

U.S.A.

GALPER IN YU I DR

INSTITUT'

USSR ACADEMY OF SCI

$117810 \mathrm{MO}$

GAMMELGAARD PETER PRDF

HANS BROGESVEJ 8

DK- 822 TER

GARDNER FRANCIS F DR

CSIRU UIVISION OF

RADIOPHYSICS

P C 76

EPPING NSW 2121

AUSTRAL IA

GARMANY CATHERINE D DR

JILA

UNIV OF COLORADO

BDULDER CO 80309

U.S.A.

GARR I SON ROBERT F PROF DAVID DUNLAP OBS

$P$ BOX 360

RICHMOND HILL

DNT 4 C $4 Y 6$

GARZOLI $S$ L DR

MARZQLI S LL DR 2412

$4 \mathrm{H}$

BUENOS AIRES

AKGENTINA
FULCHIGNONI MARCELLO PROF

$\angle A B O R A T O R I O$ DI

ASTROF ISICA SPAZIALE

E.N.R.REP PLANET OL

I TOOOO4 FRASCAT I

GAERIEL ALAN H

$A R D$

CULHAM LABORATORY

ABINGOON OXFORDSHIRE

U.K.

GAIGNEBFT J DR

AVE COPERNIC

F-06130 GRASSE

FRANCE

GALAL A A DR

HELWAN OBSERVATORY

HELWAN NEAR CAIRO

GALKIN LEONIO S A DR

CAI DANASTROPHYS I-

334413 PO NAUCHNY

CRIMEA

U.S.S.R.

GALLETTA GIUSSEPPE DR OSSERVATORIO ASTRD-

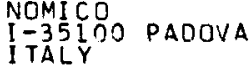

GALT JOHN A DR

OOMINION RADIO

ASTROPHYSICAL OBS

BQX 248

PENTICTON BC V2A $6 K 3$

CANADA

GAPOSCHKIN E DR

SMITHSONIAN ASTRO

OBSERVATORY

60 GARDEN ST

CAMBRIDGE MA 02138

U.S.A.

GARF INKEL BORIS DR

YALE UNIVERSITY

OBSERVATORY

NEW HAVEN CT 06520

U.S.A.

GARMIRE GORDON P PRGF

320-47 CALTECH

PASADENA CA 91125

U.S.A.

GARSTANG ROY H PROF

JILA UNIVERSITY

COLORADO

BOULDER CO 80309

U.S.A.

GASCOIGNE S C B DR

MT STROMLO

WODEN PO ACT 2606

AUSTRALIA 
GASKA STANISLAW OR

INST ITUTE ASTRONOMY

I) CHDPINA 12-18

PCLANE

GAUSTAO JZHN E PROF

EEPT OF ASTRONOMY

UNIVETSITY OF

CALIFOKNI A

BERKELEY CA 94720

$\mathrm{U} . \mathrm{S} \cdot \mathrm{A}$.

GAVRILUV I V DOR

CEIN ASTRONOMICAL THE UKRAINIAN

$\triangle C A C$ OF SCIENCES

$252127 \mathrm{KIEV}$

U.S.S.R.

GEPQIS KATHARINE \& DR

JILA

UAIVERSITY OF

C.CLCRACO

BCULLER CO 80302

U.S.A.

CEHRZ KCEERT COUGLAS DR

LEPT PHYSICS ASTRON

UA IV OF WYOMING

UA IV STA BOX 3905

LRAT IE WY 82071

U.S.A.

GENKIY IGOR L PROF DR

PFYS ICS FACULTY

KAZAKH STATE UNIV

KCNSCMCLSKAYA 96

$480512 \triangle$

SECRCELIN YVONNE M CR

CBS DE NAKSEILLE

F 13 O MARSEILLE

GERPALOI MICHELE CR

INST U ASTROPHYS IQUE

98 AIS BO ARAGO

F-75:14 PARIS

FRANCE

GERCLA HUMBERTO CR

SESELRCH CENTER

PC TOX 2 I

YCRKIWN HTS INY 10598

U.S.A.

QEYER EDWARD H PROF DR EASERVATOKIUM

HCHER LIST

C. 55 C? CAUN EIFEL

GERNAYY, F.R.

GTESRES ROSHOY AZER OR

HELWAIV OESERVATORY

HELWAY VR CAIRO

ARAQ REP. OF EGYPT

GIACHETTI RICCAREO DR

VIA BCLCGNESE 93

I $-5,01 j U$ FIREVZ

ITALY
GATEWOOD GEORGE DIRECTOR

ALLEGHENY OBS

OESERVATORY STATION

PITTSBURG PA 15214

U.S.A.

GAUT IER DANIEL

OESERVATD IRE

DE MEUDON

F-92190 MEUDON

FRANCE

GAY JEAN DR

CEKGA

AVE COPERNIC

F-O6I3O GRASSE

GEHRELS TOM PROF

LUNAR LABORATORY

UNIVER SITY OF

AR IZONA

TUC SON AZ 85721

U.S.A.

GEISS JOHANNES PROF

PHYS INST

UN IVER SITAET BERN

SIOLER STR 5

SH- 3012 BERN
SWITZERLAND

GENT HUBERT MR

35 ALBERT ROAD NORTH

MALVERN

WORCS WR 14 2TP

$U . K$.

GERARD ER IC DR

QRSERVATUIRE DE

MEUDON DEPT DE RADIO

ASTRONOMIE

F-92190 MEUCON

FRANCE

GERGELY TOMAS ESTEBAN DR

ASTRONDMY PRDGRAM

COLLEGE PK MD 20742

U.S.A.

GERSHBERG R E DR

CRIMEAN ASTROPPHYSI -

CAL OBSERVATORY

NAUCHNY

334413 CR IMEA

U.S.S.R.

GEZAR I DANIEL YSA DR

NASA-GSFC

CREENBELT MD 20771

U.S.A.

GIACAGLIA G E O PROF ESCOLA POLITECHNICA

CAIXA POSTAL 8174

SAO PAULO

BRAZIL

GIANNQNE PIETRO PROF OSSERVATORIO ASTRONDMICO

$\checkmark$ D PARCO MELLINI 84

ITALY 36 ROMA
GAUSS F STEPHEN US NAVAL OBSERVATORY WASHINGTON DC 20390

U.S.A.

GAVIOLA ENRIQUE PROF OR INSTI TUTO BALSEIRO 8400 BAR I LOCHE ARGENTINA

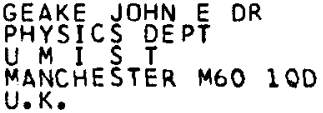

GEHREN THOMAS PH D KOENIGSTUHL

D-6900 HEI DELBERG 1

GELFREJKH GEORGIJ B DR PULKOVO OBSERVATORY

196140 LENINGRAD

U.S.S.R.

GEORGELIN YVON P DR

OBSERVATOIRE

DE MARSEILLE 2 PLACE LE VERRIER
F-13OR MARSEILLE

FRANCE

GEREAL D DR

OBS DE MEUDON

FRANCE

GERLEI OTT

HELI OPHYSICAL

4010 DEBRECEN

HUNG ARY

GETMANTSEV G G DR

RADI QPHYSICAL

RESEARCH INSTITUTE 603600 GORK I

U.S.S.R.

GHIGO FRANCIS D DR

DEPT OF ASTRONOMY

UNIV OF MINNESOTA

116 CHURCH ST SE

MINNEAPOLIS MN 55455

U. S.A.

GIACCONI RICCARDO PROF

DEPT OF ASTRONONY

GO GARDEN ST

CAMBRIDGE MA 02138

U.S.A.

GIANNUZZI MARIA A. OR

PIAZZA GRAMSCI 4

I-0:O 41 ALBANO

LALI ALE 
GIESOV DAVID MICHAEL DR PHYSICS DEPT CANPUS STATION SECCRRO NM 87801 $\mathrm{U} .5$. A.

GIESE RICHARD H PROF RUHR UNIVERS ITAET

EEPEICH EXTRATERRE-

STRISCHE PHYSIK

OEKNANY, F.R.

GILLIVGHAM PETER CR AAGLC AUSTRALIAN OBS $P$ C BOX 76

EFPIVIS NS W 2121

AUSTRALIA

GILRA DAYA P OR

KAPTEYN ASTRONOMICAL INST ITUT E

UNIVERSITY GRONINGEN GRUN I INGEN

THE NETHERLANDS

GICIA ISABELLA $M$ DR

LAP RACIOASTRONOM IA

$\checkmark I A$ IRNERIO 46

I $-40 I 26$ EOLOGNA

GIQVANNELLI RICCARDO DR CSSERVATORIO ASTROI-5 I25 FIRENZE

IT ALY

GLASER HAROLD DR

NASA

WASHINGTON DC 20546

U.S.A.

\section{GLASS IAN S DR
S A ASTRCNOMICAL DBS
P C FOX \\ CESERVATORY CP 7935 \\ SCUTH $\triangle F R I C A$}

GLEQUVA NINA I CH

INST THEORET ASTRON

USSR ACAD OF SCS

192197 LENINGRAD

$\mathrm{U} . \mathrm{S} \cdot \mathrm{s} \cdot \mathrm{k}$.

GLENCRCSS WILLIAM M OR

UAIVERSITY COLLEGE

GCWEK STREET

LCNCCN WCIE 6BT

$U . K$.

GNEVYSHEV MSTISLAV N OR IOEI ) LENIINGRAD

U.S.S.R.

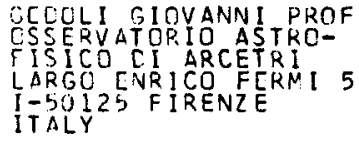

GICLAS H L MR

LOWELL OBSERVATORY BOX 1269

FLAGSTAFF AZ 86002

U.S.A.

GIESEKING FRANK DR

OBS HOHER LIST STERNWARTE BONN

D-5568 DAUN

GERMANY, F,R.

GILMAN PETER A DR

ADVANCED STUDY PROGR

NATCTR FOR ATM RES

NAT CTR
BOX 3000

BOULLER CO 80307

U.S.A.

GINGER ICH OWEN PROF

CTR FOR ASTROPHYSICS

U.S.A.

GIOYANELLI RONALD G DR

NATIONAL MEA SUREMENT

LABORATORY

PO BOX 218

LINDFIELD NSW 2070

AUSTRALIA

GIOVANNINI FONTI C

OSS ASTRONOMICO

BOLOGNA

I TAL $Y$

GLASPEY JOHN W DR

DEPT OE PHYSIQUE

UNIV DE MONTREAL

CP 612B SUCC A

MONTREAL QUE H3C $3 J 7$

CANADA

GLASSGOLO ALFRED E PROF

NEW YORK UN I VERSI TY

PHYSICS DEPT

NEW YORK NY 10003

U.S.A.

GLEDHILL JOHN A PRDF

RHODES UN I VERSITY

GRAHAMSTOW

GRAHAMSTOWN 6140

SOUTH AFR ICA

GLIESE WILHELM DR

ASTRDNOM! ISCHES

RECHEN INSTITUT

MOENC HHOF S IR 1214

GERMANY, $F \cdot R$.

GNEVYSHEVA RAISA S DR

196140 LENINGRAD

U.S.S.R.

GOOWIN J G DR

UN IV OESERVATORY

SOUTH PARKS ROAD

OXFORD OX1 3RQ

U.K.
GIERASCH PETER J DR

3 I 8 SPACE SC BLDG

1 THACA NY 14853

U.S.A.

GIETZEN JOSEPH W

ROYAL GREENWICH

OBSERVATORY

HAILSHAM

EAST SUSSEX BN27 IRP

U. K.

GILMORE ALAN C MR

101 HAPPY VALLEY RD

WELLING TON 2

NEW ZEALAND

GINZBURG VITALY L PROF LENI NSKEY PROSPECT 53

$117924 \mathrm{GSP}$

MOSCOW B 333

U.S.S.R.

GIOVANNELLI FRANCO DR LAB ASTROFISICO SPAZ $P$ B 67

I $=00044^{7}$ FRASCATI

GLAGOLEVSK I J JU V DR

CAL OBSERVATORY

USSR ACAOEMY OF SCI

$357140 \mathrm{~N}$ ARKHYZ

U. S.S.R.

GLASS BILLY PRICE DR

DEPT OF GEOLOGY

UNIV OF DELAWARE

NEWARK DE 19711

U.S.A.

GLEBOCKI ROBERT DR

INSTI TUTE OF PHYS ICS

80-308 GDANSK

POLAND

GLEI SSBERG WOLFGANG

BUCHENWEG 12

D-6374 OBERURSEL

GERMANY, F.R.

GLUSHNEVA I N DR

STERNBERG STATE ASTRONOMICAL INSTI TUTE $117234 \mathrm{MOSC}$ OW

U.S.S.R.

GODART QDON PROF

RUE OE CHATEAU 96 BELGIUM ROUSVAL

GOEBEL ERUST DR

INST F ASTRONOMIE

UNI VERSITAEI WIEN

$A-1180$ WIEN

AUSTRIA 


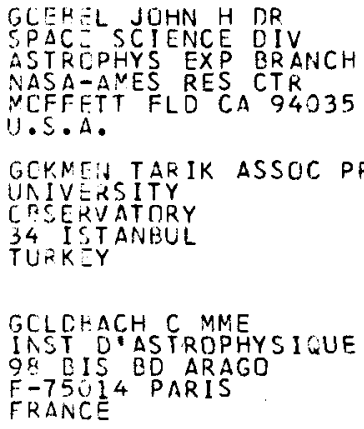

GCLOSMITH DONALD H. DR. 2153 RUSSELL STREE U.S.A.

\section{GCLCSTEIN RICHARD M OR JET PROPULS ION \\ LAUCRATCRY \\ 48.90 OAK GROVE CRIVE \\ PASADENA CA 91011 \\ U.S.A. \\ GCLDWIRE HENRY C JR \\ UNIVEXSITY OF \\ CALIFURNIA LASL \\ $B C X 1663$ MS 420 \\ LES ALAMOS NM 87545 \\ U.S.A. \\ GOMES ALERCID M DR \\ $R$ GAVIAC PEIXOTO 13 \\ $\angle P 1431$ \\ ICARAI $24000^{\circ}$ \\ NITEROJ ERJ \\ BRAZIL}

GCNDHALEKAR PRAEHAKAR DR

14 REDWCOD CLOSE

SCUTHMCOR

CIXCN OXI3 5DH

U.K.

GCNLE RCGER F J IR

DESERVATCIRE ROYAL

AVE CIRCULAIRE 3

E- II 85 BRUXELLES

BELGIUM

GOPALA RAU U V MR

ASTRCPHIYSICAL OBS

KCEIA IKANAL 3

INOIA

GCRCCN CHARLOTTE PROF

5 SQUARE PORT-ROYAL

F-75 13 PAR I

FRANCE

GCRDCN KURT ISS J PROF

HAMPSHIRE COLLEGE

U.S.A.
GOKDOGAN NUZHET PROF

UNIVERSITY

UNIV ISTANBUL

ISTANBUL

TURKEY

GOLAY MARCEL PROF

OBSERVATOIRE

DE GENEVE

CH- I290 SAUVERNY GE

SWITZERLAND

GOLDBERG LEO PROF

KITT PEAK NATIONAL

OBSER VATCRY

TUC SON AZ 85726

U.S.A.

GCLDSMITH PAUL F DR

DEPT PHYSICS ASTRON

GRC TOWER B RM 626

UN IV OF MASS

AMHER ST MA D 1003

U.S.A.

GOLDSTEIN SAMUEL J PROF

UNIVERSITY VIRGINIA

PO BOX 3818

CHARLOTTESVILLE

VA 22903

U.S.A.

GOLLNOW H DR

MT STROMLO OBS

AUSTRALIA

GOMEZ GONZALEZ J DR PASEO IMPERIAL $296 \mathrm{H}$ MADR ID 5

SPAIN

GONDOLATSCH FRIERICH PROF ASTRONOMISCHES MOENCHHOFSTR 12-14 $D-6900$ HEIDELBERG GERMANY, F.R.

GOODY R M

CEPP

PIERCE HALL

29 OXFORD STREET

CAMBR IDGE MA 02138

U.S.A.

GOPASYUK $S$ I DR

CRIMEAN ASTROPHYSI-

CAL OBSERVATORY USSR ACAD OF SCI NAUCHNIY 334413 CR IMEA U.S.S.R.

GORDON COURTNEY P PROF HAMPSHIRE COLLEGE U.S.A.

GORDON MARK A DR NAT RADIO ASTRONOMY QBS SUITE 100 $2010 \mathrm{~N}$ FORBES BLVD TUCSON

U.S.A.
GOKHALE MORESHWAR HAR I DR INDIAN INSTI I TUTE OF BANGALDRE 560034 I NDI A

GOLD THOMAS PROF SPACE SCIENCE BLDG CORNELL UNIVERS U. S. A.

GOLDREICH P DR

CALTECH

PASADENA CA 91109

U. S.A.

GOLDSMITH S. DR . DEPT OF PHYSICS AND ASTRONOMY

TEL AVIV UNIVERSITY TEL AVI

GOLOSWORTHY FREDERICK A SCHOOL MATHEMAT ICS

UNI VERSITY OF LEEDS

LEEDS LSL 9 JT

GOLUB LEON DR

HAR VARD COLLEGE

OBSERVATORY

60 GARDEN ST

CAMERIDGE MA 02138

U.S.A.

GOMEZ MARIA THERESA DR DSSERVATORIO ASTRONOMICO NAPOLI

GONZALEZ G NAT ASTROFIS INST NAC DE ASTROF I
OPT Y ELECTRONICA
APT POSTALES 21651 PUERLA PUE MEXICD

GCOSSENS MARCEL DR LOZENHOEKSTRAAT 127 BELGIUM

GORBATSKY VITALI J G PROF

LENINGRAD UNIVERSITY

OB SERVATORY

I 991 I B LENINGRAD

U.S.S.R.

GORDON I $M$ DR

INSTI TUTE OF RADIO

PHYSICS AND

ELECTRONIC

310085 KHARKOV

U.S.S.R.

GORENSTEIN PAUL DR

CENTER FOR ASTRO-

PHYSICS

60 GARDEN STREET

CAMBRIDGE MA 02138

U.S.A. 


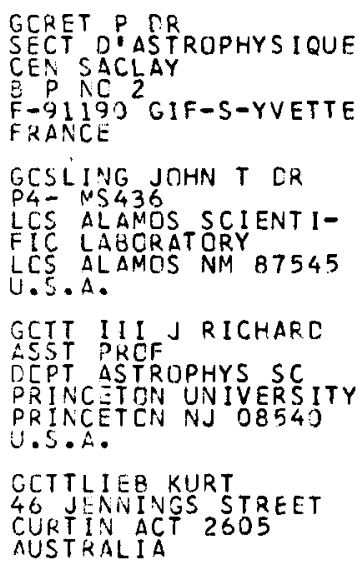

GCUGUENHEIM LUCIENNE CESEKVATDIRE CE MEUDON RAOI CASTRONOMIE F-92190 MEUDON FRANCE

GCWER J F R DR

IE 15 MCTAVISH ROAD R L

GRAECSKE HAROLC C JR LAWRENCE LIVERMORE LAPCRATORY

$P C E C X 808$

LIVESUCRE CA 94550

U.S.A.

GRAHAN ERIC DR

HICH ALTITUDE OES

NATL CENTER ATMO RES

$P C$ ACX 3000

RCULLER CO 80307

U.S.A.

GRAINGER J F DR

UN IST

NANCHESTER MGO 100

U.K.

GKASCALEN GARY L CR LNIV OF WYOMING $P C$ COX 3905 UN STA LARAMIE WY 8207 U.S. A.

GRAYLECK EDWIN J ER PHYSICS DEPT

UAIV UF NEVADA

4555 S MARYLAND PKY

LAS VECAS NV 89154

U.S.L.

GREEN LOUIS C PROF

HAVERFCRO COLLEGE

HAVEKFCRD PA 19041

U.S.. .
GORGOLEWSKI STANISLAW DR

INST OF ASTRONOMY

NIC COPERNICUS UNIV

CrOP INA $12-18$

\begin{tabular}{l} 
POLAND \\
\hline
\end{tabular}

GOSS $W$ MILLER DR

KAP TEYN ÁSTRONOMICAL

INSTITUTE

POSTBUS 800

9700 AV GR ONINGEN

THE NETHERLANDS

GOTTESMAN STEPHEN T DR

PHYS ICS AND

ASTRONOMY

UNIVERSITY FLORIDA

GAINESVILLE FL 32611

U.S.A. GOUDAS CONSTANTINE L PRCF UNIVERSITY OF PATRAS

GOULD RDBERT J PROF PHYSICS DEPT

UCSO

UA.S.A.LA CA 92093

GOY GERALD DR

OBSERVATOIRE

DE GENEVE

CH- 1290 SAUVERNY

SWITZERLAND

GRABOWSKI 8QLESLAW DR

INSTITUTE OF PHYSICS

UL DLESKA 48

45951 OPOLE

POLAND

GRAHAM JOHN A DR

CERRO TOLOLO

CASILLA 63 D

LA SERENA

CHILE

GRANDI STEVEN ALDRIDGE DR

DEPT OF ASTRONOMY

MATH SCS BLDG

UNIV OF CALIFORNI

LOS ANGELES CA 9 CO2 24

-S.A.

GRATTON LIVIO PROF

LABDRATOR IO DI

CAS POST 67

I-O0044 FRASCATI

ITALY

GREBENIKOV E A PROF DR

INSTITUTE OF THEORE-

TICAL AND EXPERIMEN-

117259 MOSCOW

U.S.S.R.

GREEN RORIN M OR

GLASGOW UN IVER SITY

GLASGOW GI2 8QQ

U.K.
GOSACHINSKIJ I V OR

SAN

196140 LENINGRAD

U.S.S.R.

GDSSNER SIMONE D MRS

NEW YOSOTH STREET

U. S. A.

GOTTLIEB CARL A OR

GODDARD INST FOR

SPACE STUDIES

2880 BROAD WAY

NEW YORK NY 10025

U.S.A.

GOUGH DOUGLAS OO DR

INST OF ASTR

CAMBRIDGE CB3 OHA

U.K.

GOWER ANN C DR UNI VERSITY OF VICTO-

RIA OBSERVATORY

VICTORIA BC VBW $2 Y 2$
CANADA

GOYAL A N DR

DEPT OF MATHEMATICS

UNI $V$ OF RAJASTHAN

JAI PUR

INDIA

GRADSZTAJN E DR

INSTI TUT DE PHYSI QUE NUCLEAIRE D-ORSAY

FRÁNCE

GRAHL BERND H OR MPI FUER RAOIOASTRONOMIE

AUF DEM HUEGEL 69

D-5300 BONN

GERMANY, F.R

GRANT I AN $P$ DR

PEMBROKE COLLEGE OXFORD OXI 1 DW

U.K.

GRAY DAVID F PROF

DEPT OF ASTRONOMY

UNI VERSITY OF

WESTERN ONTARIO

LONDON ONT NGA 589

CANADA

GREEN JACK PROF

DEPY OF GEQLOGY

SI TY-LDNG BEACH LONG BEACH CA 90840

U.S.A.

GREENBERG J MAYD DR

HUYGENS LABORATORY

UNI VERSI IY OF LEIDEN

WASSENAARSEWEG 78

2300 RA LEIDEN

THE NETHERLANOS 


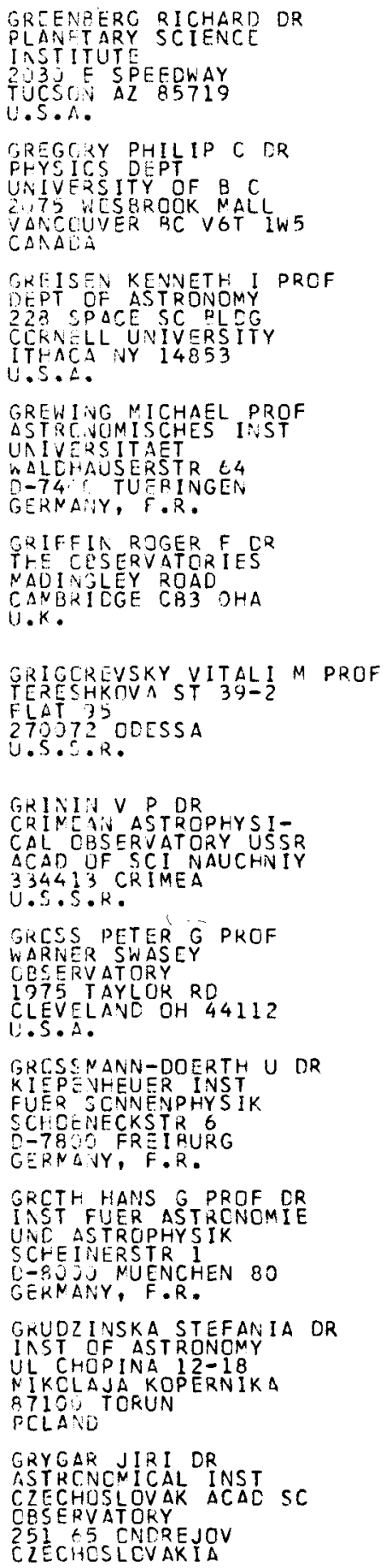

GRYGAR JIRI OR ASTRCNCMICAL INST CBSERVATORY 251 t5 CNCREJOV CZECHCSLCVAKIA

GREENSTEIN GEORGE PROF

AMHER ST COLLEGE

AMHER ST MA 01002

U.S.A.

GREGORY STEPHEN ALBERT DR SICS DEPT

BOWLING GREEN STATE

UNIVERSITY BOWLING GRN OH 4340

BOWLING

GRENON MICHEL DR

DES DE GENEVE
CH-1290 SAUVERNY

CHO 1290 SAUV
SWITLERLAND

GREYBER HOWARD D DR

IOI23 FALLS ROAD

$U . S \cdot A$.

GR IFFITH JOHN S PROF

DEPT OF MATH SC ST TTY

THUNDER BAY

ONT P7B 5 E

CANADA

GR IGOR JEV VICTOR M DR

SIBERIAN INST OF TER MAGN, IONOSPH,RAD WP $P$ BOX

664697 IRKUTSK

U.S.S.R.

GR ISHCHUK L P DR STERNBERG STATE ASTRONOMICAL INSTI TUTE 117234 MOSCOW

U.S.S.R.

GROSSMAN ALLEN S PROF ERW IN FICK

IOWA ST UN IVER SITY

AMES IA 50011

U.S.A.

GROTEN ERWIN PROF

IIST F PHYSIKALISCHE

GEODAESIE

PETER SENSTR 13

D-GIOO DARMSTADT

GERMANY, F.R.

GRURISSICH C PROF DR

VIA AOSTA $34 / 5$

ITALY 35 ITO PADOVA

GRUEFF GAVR IL DR

LABORATOR IO DI RADIO

ASTRONOMIA C N R

$\checkmark$ IA IRNER IO 46

ITALY 26 BOLOGNA

GRZEDZIELSK I STANISLAW DR CENTER FOR SPACE RES UL BARTYCKA 18

POLAND
GREENSTEIN J L PROF HALE OBSERVATORIES CALIF INST OF TECHN PASADENA CA 91125 U. S:A.

GREGUL A YA DR THE

KIEV UNI VERS ITY

QRSERVATORNAYA 3

$252053 \mathrm{KIEV}$ )

GREVESSE N DR

INSTITUT

D ASTROPHYS I QUE B-4200 COINTE-OUGREE

GRIFFIN RITA E M DR

THE OBSERVATORIES

MADINGLEY ROAD

CAMBRIDGE CB3 OHA

U.K.

GRIFFITHS RICHARD E DR

HARVARD SMITHSONIAN

CTR FOR ASTROPHYSICS

60 GARDEN STREE

CAMBRIDGE MA OLI 138

U.S.A.

GRINDLAY JONATHAN E DR HARVARD SMITHSONIAN

CENTER FOR ASTROPHYS

60 GARDEN ST

CAMRRIDGE MA O213B

U.S.A.

GROSBOL PREBEN JOHNSON DR

COPENHAGEN UNIV

OBSERVATORY

OSTER VOLDGADE

DK-1350 COPENHAGEN-K

DENMARK

GROSSMAN LAWRENCE PROF

DEPT GEOPHYSICAL SC

UNI VERSITY CHICAGO
5734 SOUTH ELLIS AVE

CHICAGO IL 60637

U.S.A.

GROTH EOWARD J III

PHYSICS DEPT

PADWIN HALL N

PRINCETON NJ OBS40

U.S.A.

GRUDLER PIERRE

AVE COPERNI

AVE COPERNIC

FRANCE

GRUSHINSKI J $N$ P PROF DR

STERNBERG STATE AS-

TRONOMICAL INSTITUTE

117234 MOSCOW

U.S.S.R.

GUARNIERI ADRI ANO DR

OSSERVATORIO

ASTRONOMICO

VIA ZAMBONI 33

I 40126 BOLOGNA 
GUEANOV VADIM S TR PULKEJC CESERVATORY 19614 LENIVGKAC

L.5.5.2.

ASENIZLL-LINGNER U OR RECH INSTITUT

MCECHHCTSTR I $2-14$

DO-ES HEDDELREKC

GEKN $\Delta M Y, F \cdot R$.

CUEST JCHA: E CR

UNIVERSITY OF LCNCOV

MESERVATORY

LENCEA NWT $2 Q S$

U.K.

GUIAAN ERWARD FRANCIS DR OFT UF ASTRUNOMY

VILLANCVA UIUIV

VILLATCVA PA 19085

U.S.A.

SULL STEPHEN J FNOS CR

CANESILOSE

U.K.

SULYAEV A P DR

LNIVERSITETSKIJ

PKCSDEKT I3

$1: 7234 \mathrm{NOSCOW}$

U.S.S.R.

GURN HAROEN S CR

ANC SPACE SCIENCES

PUAJABI U:VIVERSITY

PATIELA 147002

INUIA

GURTLVEVKO E A DR

NAIN ASTRCNOMICAL

CF SCIENCES

U.S.S.R.

GUEF JNCV H EH CR

CAL OHSERVATORY

373243 AZERBAICZAN

U.S.S.R.

GUTH VLADIMIR CR PRDF

ASIRCUCMICAL INST

Z5 to ONDREJOV

CPSEXVATCRY

CLECHCSLCVAKIA

GYLDENKERNE KJELD DR

CCFENHAGEU UNIVER-

SITY OBSERVATORY

?RCRFELD

$(K=434)$ TOLLOSE

DENNARK

HACINOH J DR

STERREW ACHT

2. 3 C RA LEIOEN

THE NETHERLANDS
GULUR N DF

EGE UN I VERS I TY

OHSER VATORY

P.K. 21 BORNOVA

I $2 N I R$

GUER IN PIERRE DR

INST D ASTROPHYSIQUE

98 B IS BL VD A

FRANCE

GUIBERT JEAN DR

OBSERVATOIRE

DE MEUDON

F-92190 MELDON

FRANCE

GUINUT BERNARD R PROF BUREAU INTTERNATIONAL DE L HEURE

GI AV L OESERVATOIRE $F=75014$ PAR IS

FR $\triangle N C E$

GULL THEOURE R DR

CCLE $683^{\circ}$

GONDARD

SPACE FLIGHT CENTER

GREEIVEELT MD 20771

U.S.A.

GULYAEV RUDOLF A DR

IZMIRAN

AKAOEMGORODOK

142092 MOSCOW REGION

U.S.S.R.

GUK SHTEIN A A DR

SPACE RESEARCH

USSR ACADEMY OF SCI

i1 7810 MOSCOh

U.S.S.K.

GURZADIAN G A PROF DR BYURAKAN $A$ STROPHYSICAL DBSER VATORY

378433 ARMENIA

U.S.S.R.

GUSSMANN EA DR

ZENTRAL INST ASTROPHY

ASTROPHYSIKALIS OBS

TELEGRAFENBERG

$C Q R=15$ POTSDAM

$G \cdot D \cdot R$.

GUTHR IE BRUCEN G DR

ROYAL OB SERVATORY

BLACKFORD HILL

EC IN BURGH

EOINBURGH EH9 $3 \mathrm{HJ}$

U.K.

HAAS J PROF DR

D- 5300 BONN
GERMANY, F.R.

HACHENBERG OTTO PROF DR RADIOASTRON INST DER UNIV BONN

$\triangle U F$ DEM HUEGEL 71

DESMOC BONN
GUELIN MICHEL CR

MEUOON

F-92190 MEUDON

GUERRERO GIANANTONIO DR OSSERVATORIO ASTRO-

NOMICO

MITANO

GUIDICE DONALD A DR

A F SEDPHYSICS

$\angle A B O R A T O R Y$

HANSCOM AFB MA 01731

U.S.A.

GULKIS SAMUEL DR

JET PROPULSIONA

LABORATORY

4800 OAK GROVE DRIVE

PASADENA CA 91103

U. S.A.

GULMEN OMUR OR

EGE UNI VERSITY

OBSERVATORY

PK 21

BORNOVA IZMIR

GUNN JAMES E PROF

CALTECH

1 $05-24$

PASADENA CA 91125

U.S.A.

GURSKY HERBERT DR

CENTER FOR

ASTROPHYSICS

6OGARDEN STREET
CAMBRIDGE MA O2I38

U.S.A.

GUSEINOV O KH OR

SHEMAKHA ASTROPHYSI -

CAL OBSERVATORY

373243 AZERBAIDZAN

U.S.S.R.

GUSTAFSSON BENGT DR

ASTRONOMICAL

DBSERVATORY

BOX 515
S-75 120 UPPSALA
SWEDEN

GUTIERREZ-MORENO A DR MRS

DEP DE ASTRONOMI A

UNI VERSIDAD DE CHILE

CASILLA 36-D

SANTI AGO

CHI LE

HABIBULLIN SH T PROF DR

ENGELGAROT ASTRONO-

MICAL OBSERVATORY

422526 KAZAN

U.S.S.R.

HACK MARGHERITA PROF

OSSER VATORIO

ASTRONOMICO

VIA TIEPOLO 


\section{HACKWELL JOHN A DR CEPT PHYSICS ASTRON UAIV OF WYOMING LARAMI \\ U.S.A.}

11DLJIDEMETRIOU JOHN CPROF EEPT GF THEORET ICAL MECHANICS

UA IV OF THESSALONIK I THESSALCNIKI

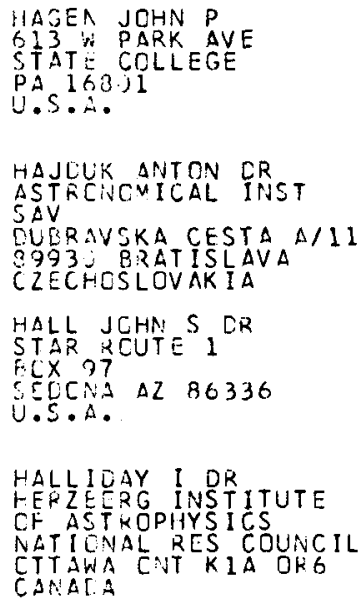

HANEEV ANTILLA KADRLE A

PRCF ÜR

UAIVERSITY OF OULU

FIA:LARC

HANNERSCHLAG ROEERT H DR IR

ACTRE VCM I CAL INST

SERVAAS

THE NLTIIERLANCS

HAAASZ JAN OR ASTRCNCMICAL CENTER UL CHOPINA I27I8 NICAL CENTREPAN 971\%:T CRUN

HANNER MARTHAS DR

MEL $1 \& 3-301$

PASGTENA CA 91103

U.S.A.

HANSEA RICHARE T MR

HIGT ALTITUOE

PESERVATORY 3000

HCULLER CO 80303

U.S.A.

HAPKE PRUCE W DR

TEPT EARTH PLANET SC

3 CLE ENOIN HALL

PITTSEURGH PA 15235

L.S.A.
HACYAN SHAHEN DR.

GROUPE D ASTROPHYSI QUE RELATIVISTE

F-92190 MEUDON FRANCE

HAEFNER REINHOLD DR

UN IV STERNWARTE

SCHE INER STRASSE 1

D-80OO MUENCHEN 80

GERMANY, F.R.

HAGEN-THORN V A DR 10 LINIJA 33 199178 LEN INGRAD

U.S.S.R.

HALL DONALD iN DR KITT PEAK NATIONAL OBSERVATORY

P U 26732

TUC SON AZ 85726

U.S.A.

HALL R GLENN DR US NAVAL OBSERVATORY WASHINGTON DC 20390

U.S.A.

HAMADA TETSUO PROF DEPT OF PHYSICS IBARAK I UNIVER SITY 310 MITO

JAPAN

HAMID S EL DIN DR

FACULTY OF SCIENCE

UNIVERSITY FOUAD

GIZA CAIRO

ARAB REP. OF EGYPT

HAMMER SCHLAG-HENSBERGE

$G$ DR

ASTRONOMICAL INST

RDETER STRAAT IN

1018 WE AMSTERDAM
THE NETHERLANOS

HANBURY RROWN R PROF SCHOOL OF PHYSICS SYDNEY NSW 2006

AUSTRALIA

HANSEN CARL J PROF

JILA

UNIVERSITY OF

BCLULDAR CO 80309

U.S.A.

HANSDN ROBERT B DR

LICK OBSERVATORY

UNIV OF CALIFORNIA

SANTA CRUZ CA 95064

U.S.A.

HARA KEN NOSUKE DR SFNDAI - SHIRIT SU-JDSH SENIOR HIGH SCHOOL KASHIWAG 3-3-1 SENDAI $980^{\circ}$ JAPAN
HADDOCK FRED T OR

UNIV OF MICHIGAN 937 PHYSICS-ASTRONOMY PLDG ANN ARBOR MI 48104 U.S.A.

HAERENDEL G DR MPI FUER PHYSIK UND ASTROPHYSIK INST EXTRATERR PHYS D-BO46 GARCHING GERMANY, F.R.

HAGFORS T DR

MIT LINCOLN LAB MILLSTON/HAYSTACK LEXING

HALL DOUGLAS S DR

VANDERBILT

UNIVERSITY

NASHVILLE TN 37235

U.S.A.

HALLAM KENNETH L DR

CODE 685 LAB FOR

ASTRON SOLAR PHYSICS

GSFC

GREENBELT MD 20771

U.S.A.

HAMDY M A M DR

HELWAN OBSERVATORY HELWAN

ARAB REP. OF EGYPT

HAMILTON P A DR

UNIV QF TASMANIA

HOBART TASMANIA AUSTRALIA

HAMZAOGLU ESAT E H DR

FEN FAKULTES

GOK BILIMLERI ENST BORNOV

TURKEY

HANES DAVID A LR

INST OF ASTRONOMY

UNIV OF CAMBRIDGE

CAMBRIDGE

U.K.

HANSEN LEIF LECTURER COPENHAGEN UNIVERSI TY OESERVATORY OSTERVOLDGADE DK- 1350 COPENHAGEN K DENMARK

HANSSON NILS DR

LUND OBSERVATORY

SVANEGATAN 9
$5-222 \quad 24$ LUNO

STI2 22

HARA TETSUYA DR

KYOTO SANGYO UNIV KYOTO 603

JAPAN 


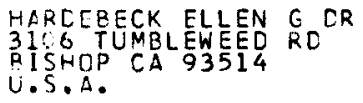

HARDCRP JOHANNES PROF DEPT EARTH SPACE SC STATE UNIVERSITY STCNY BRCOK NY 11794 U.S.A.

HARMER CHARLES F W MR RCYAL GREENWICH OBS HAI LSHAM

E SUSSEX BN27 1RP

U.k.

HARC GUILLERMO CR

IAST NAC ASTROFISICA CPT ICA Y CLECTRON ICA $A P 216 Y-51$

PUERLA PUE

HARRIS ALAN WILLIAM DR NPL $204-72$,

$48 \% 0$ OAK GROVE CRIVF PASACENA CA 91103 U.S.A.

HARKIS WILLIAM E DR

OEPT OF PHYS ICS

RCNASTER UNIV

CANACA

HART MICHAEL H DR

PHYS ICS DEPT

TKINITY UNIV

SAN ANTONIO TX 78284

U.S.A.

HARTLEY KENNETH F DR

RCYAL GREENW ICH

CESERVATORY

HAILSHAM

EAST SUSSEX BN27 IRP

U.K.

HAKTLICKK DAVIO A DR

UN IVERSITY VICTORIA

ICPT DF PHYS ICS

$\checkmark I C T C R I A B C$ VBW $2 Y Z$

CANAEA

HAKV:Y GALE A CR

INST F GESCHICHTE D

NATURW ISSENSCHAFTEN

GCETHE UNIVERS ITAET

6000 FRANKFURT

GERMANY, F.R.

HARWIT MARTIN PROF

SPACE SCIENCE BLEG

CERNCLL UNIVERS ITY

ITHACA NY 14853

U.S.A.

HASER LEO N K DR

YPI FUER EXTRATERRE-

STRISCHE PHYSIK

-2046 GARCHING

CERNANY, F.R.
HARDIE R PROF

DYER OBSERVATORY

VANDERB ILT

UN IVER S ITY

NASHVILLE TN 37235

U.S.A.

HARDY EDUARDO DR PH

DEPT DE PHYSIQUE

UNIVERSITE LAVAL

OUC DES SCS GENIE

QUEBEC

HARMER DIANNE L MRS

ROYAL GREENWICH OBS

HER S TMONCEUX CASTLE

HA IL SHAM

E SUSSEX BN27 IRP

U.K.

HARR INGTON J PATRICK DR

ASTRONDMY PROGRAM

UN IVER S I TY MAR YLAND

COLLEGE PK MD 20742

U.S.A.

HARR IS DANIEL E DR

DRAO

P O BOX 248

PENTICTON BC VZA $6 K 3$

CANADA

HARR ISON EDWARD. R PROF

DEPT DF PHYSICS AND

ASTRONOMY

UNIVERSITY OF MASS

AMHERST MA 01003

U.S.A.

HARTEN RONALO H DR

RADIOSTERRENWACHT

OWINGELOO 7514

THE NETHERLANDS

HARTNER WILLY R PROF

UN IVER S I TAE T

D-600O FRANKFURT

GERMANY, F.R.

HARTZ THEODORE $R$ DR

COMMUN ICATIONS

RESEARCH CENTER

POB 11490 STATION H

OTTAWA ONT K2H BS2

CANADA

HARVEY J W DR

KITT PEAK NATIONAL

OPSER VATORY

950 N CHERRY AVE

TUCSON AZ 85726

U.S.A.

HASEGAWA HIROICHI DR

DEPT OF PHYSICS

KYOTO UN I VER SI TY

SAK YO-KU

KYOTO 606

JAPAN

HASLAM C GLYN T DR

MP I F RADIO

ASTRONOMIE

AUF DEM HUEGEL 69

D-5300 BONN
HARDING GEORGE A

ROYAL GREENWICH

OBSERVATORY

HAILSHAM

EAST SUSSEX BN27 IRP

U.K.

HARMANEC PETR DR

ASTRONOMICAL INST

CLECHOSLOVAK ACAD

OF SCIENCES

25165 ONDRE JOV

CZECHOSLOVAKIA

HARMS RICHARD JAMES DR

PHYSICS DEPT B-OI9

UNIV OF CALIFORNIA

SAN DIEGO

LA JOLLA CA 92093

U.S.A.

HARR INGTON ROBERT S DR

U S NAVAL

CBSERVATORY

WASHINGTON DC 20390

U. S.A.

HARR I S GRETCHEN L H DR PH DEPT OF PHYSICS

UNIV OF WATERLOD

WATERLOO DNT

CANADA

HARROWER GEORGE A DR

LAKEHEAD UNIVERSITY

THUNDER BAY

QNT P7B $5 E I$

CANAOA

HARTL HERBERT DR

INST F ASTRONOMIE

UNIVERSITAETSSTR 4

$A-6020$ INNSBRUCK

AUSTRIA

HARTOOG MARK RICHARD DR

LICK OBS

UNIV OF CALIFORNIA

SANTA CRUZ CA 95064

U.S.A.

HARVEY CHRISTOPHER C DR OBSERVATOIRE

DE MEUDON

F-92190 MEUDON

FRANCE

HARVEY PAUL MICHAEL DR

STEWARD OBS

UNI $V$ OF ARIZONA

TUC SON AZ B5721

U.S.A.

HASEGAWA ICHIRC DR

2-3-II SAIDAIJINO-

GAMI

NARA 631

JAPAN

HASSAN S M DR

HELWAN INST OF ASTRO

NOMY AND GEOPHYS ICS

HELWAN NEAR CAIRD

$A R A B$ REP. OF EGYPT 
HATTCRI AKIRA A PROF HILA OESERVATORY

KAMITAKARA

GIFU KEN 50613

$J \triangle F A N$

HAUG ULRICH PROF

HANPURGER STERNWARTE

GCJENBERGSWEG 112

[-2) 53 HAMEURG 80

GEKMAIYY, F.R.

HAUPT RALPH F
317 ACEERLEY COURT
SILVER SPRING
MC 2 906

C 2,926

\section{HAVNES OVE DR \\ AURCRAL GBSERVATORY \\ LAIVERS ITY OF TRDMSO \\ $N-3030$ TROMSO \\ ALRWAY \\ HAWKINS G S DR \\ WE GARDEN ROAD 2181 \\ U.S.A.}

HAYES DCNALD S CR

TUCSCI AZ 85719

U.S.A.

\section{HAYAES RAYMOND F PROF \\ CSIRC \\ UIV CF RAOIOPHYSICS \\ PF DEX 76 \\ 作}

HAZLEHURST J DR

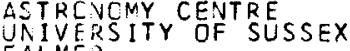

$F A L N[R$

CRIGHTDN BNI 9GH

U.K.

HEARINSHAW JOHN F DR

OEPT OF PHYSICS

UN IVERS ITY

CFRISTCHURCH

NEW LEALAND

HECELE DOUGLASW O PROF

CEPT OF PHYSICS

RCYAL HOLLOWAY

CCLLEGE

ECHAN SURREY TWZOOEX

HEFELE HERBERT PH D

KCNICSTUHL

C. -6909 HEIDEL BERG I

GERNANY, F.R.

HEGYI DENNIS J ASS PROF

RANCALL LABORATORY

UAIVERSITY OF

NICHISON

ANA $\triangle R B C R$ MI 48109

U.S.A.
HAUCK BERNARD PROF

INST D ASTRONOMIE

UN IVER SITE LAUSANNE

CH- 1290 CHAVANNES

DES BOIS

SWITZEKLAND

HAUGE OIVIND DR

INSTITUTE OF THEORE-

TICAL ASTROPHYSICS

UN IVERSITY OF OSLO

BLINDERN USLO 3

NORWAY

HAUPT WOLFGANG OR

GIRONDELLE 105

D-4630 BOCHUM

GERMANY, F.R.

HAWARDEN T G OR

UK 48 INCH SCHMIDT

TELESCOPE UNIT

PRIVATE BAG

COONABARAN NSW 2857

AUSTRALIA

HAYAKAWA SACHIO PROF

DEPT OF PHYSICS

NAGOYA UNIVERSITY

FUROKO CHIKUSAKU

NAGOYA 464

JAPAN

HAYLI AVRAM PROF OBSER VATOIRE DE LYON F-69230 ST CENIS

LAVAL

FRANCE

HAZARD CYKIL DR

INST OF ASTRONOMY

MADINGLEY ROAD

CAMBR IDGE CB 3 DHE

U.K.

HEAP SARA R DR

CODE 672 GODDARD

SPACE FLIGHT CENTER

GREENBELT MD 20771

U.S.A.

HECK ANDRE DR

VILLAFRANCA SATEL-

APARTADO 54065

MADR ID

SPAIN

HEDEMAN E RUTH

MCMATH HULBERT

ORSER VATORY

$895 N$ LAKE ANGELUS RD

PONTIAC

HEFFERLIN RAY A PROF

PHYSICS DEPT A

DRAWER H

COLLEGEDALE TN 37315

U.S.A.

HEIDMANN JEAN DR

OES DE MEUDON

F-92190 MEUDON
HAUG EBERHARD DOZ DR

MOZARTSTRASSE 20

D-7418 METZINGEN

GERMANY, F.R.

HAUPT HERMANN F PROF

ASTRONOMISCHES INST

DER UNIVERSITAET

UNIVERSTAETSPLATZ 5

$A-8010$ GRAZ

AUSTRIA

HAVLEN ROBERT J DR

NAT RADIO ASTRON OBS

EDGEMONT ROAD

CHARLOTTESVL VA2290I

U.S.A.

HAWKING STEPHEN W PROF

DEPT APPLIED MATHS

AND THEOR PHYSICS

SILVER STREET

CAMBRIDGE CBO OEW

U.K.

HAYASHI CHUSHIRO PROF

PHYSICS ÖEPT

FACULTY OF SCIENCE

KYOTO UNI VERS ITY

KYOTO 606

JAPAN

HAYMES ROBERT C PROF

DEPT OF SPACE PHY -

RICE UNIVERSITY

HOUSTON TX 77001

U.S.A.

HAZER $S$ DR

FACULTY OF SCIENCE

DEPT OF ASTRONOMY

$P K 21$

BORNOVVA IZMIR

TURKEY

HEARN ANTHONY G DR

STERREKUND IG INST

SERVAAS BOLWERK 13

UTRECHT

THE NETHERLANDS

HECKMANN OTTO PROF OR

WDHNSTIFT GOETTINGEN

$A P P$ B 1403

CHARLOTTENB.STR 29

D-340O GOETTINGEN

GERMANY, F.R.

HEESCHEN DAVID S DR DIR NAT RADIO ASTR OBS

EDGEMONT

CHAR LOTTESVI LLE

VA 22901

U.S.A.

HEGGIE DOUGLAS C DR

UNIV OF EDINBURGH

ED I NBURGH EHO $3 \mathrm{JZ}$
U. K.

HEIDMANN NICOLE DR

INST D ASTROPHYSIQUE

98 BIS BLVO ARAGO

F-750I4 PARIS 
HEILES CARL PROF

$\triangle S T R C N C M Y$ DEPT

UNIVERSITY DF

CALIFORNIA

FERKELEY CA 94720

U.S.A.

HEINTLE J R W DR

SCANTEORCH

SERVAAS BOLWERK 13

UTRECHT

THE NETHERLAINCS

HEKELA JAN OR

ASTRC ICMICAL INST

25165 CNDREJOV

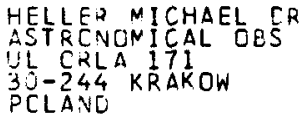

HELT RODIL E

UNIVERSITY

CSTER VOLCGADE

DK-135\% COPENHAGEN

DENMARK

HENLE KARL G ASTRONAUT

JCHNSON SPACE CENTER

HCUSTON TX 77058

U.S. A.

HENRARO JACQUES PROF

FAC UNIV OE NAMUR

t. I RUE DE BRUXELLES

EELGIUN NAMUR

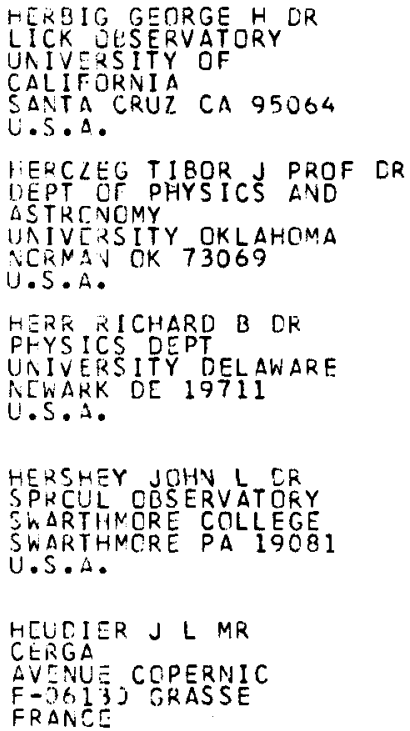

HEILESEN POUL MARTIN DR BOULEVARDEN 14216 CK- 2800 LYNGBY DENMARK

HEI'SE JOHN OR

作

3527 HS UTRECH

THE NETHERLANDS

HELALI YHYA E DR

HELWAN OBSERVATORY

HELWAN-CAIRO

$A R A B$ REP. OF EGYPT

HELLWIG HELMUT WILHELM DR NAT BUREAU STANDARDS

TIME \& FREQ DIV

325 BROADWAY RM 2031

BOULDER CO 80303

U.S.A.

HEMENWAY CURTIS L PROF
DUDLEY DBS PLAZA 7

1202 TROY

SCHENECTADY RD

LATHAM NY 12110

U.S.A.

HENON MICHEL C DR

OESERVATOIRE DE NICE

B P 252

F-06007 NICE CEDEX

FRANCE

HENR IKSEN RICHARD N DR

ASTRONOMY GROUP

DEPT OF PHYSICS

QUEENS UN IVERSITY

KINGSTON ONT KTL $3 N G$

CANADA

HERBST ERIC DR

DEPT OF CHEMISTRY

CLG WILLIAM \& MARY

WILL IAMSBG VA 23185

$U . S . A$.

HER GET PAUL DR

3522 HER SCHFL VIEW

$U . S . A$.

HERRMANN DIETER DR

ARCHENHOLD OBS

AND PUBLIC OBS

1193 BERL IN-TREPTOW

$G \cdot D \cdot R$.

HER ZBERG GERHARD DR

HERZBERG INSTITUTE

OF ASTROPHYSICS

NAT RES COUNCIL

OTTAWA ONT KIA OR6

CANADA

HEWI SH ANTONY PROF

CAVENDISH LABORATORY

MADINGLEY RD

CAMBR IDGE CB 3 OHE

U.K.
HEINTZ WULFF D DR

SWAR THMORE COLLEGE

SWARTHMORE PA 19081

U.S.A.

HEISER ARNOLD $M$ DR

DYER OBSERVATORY

VANDERBILT UNIV

BOX $1803-S T A$ B

NASHVILLE TN 37235

U.S.A.

HELFER H LAWRENCE PROF DEPT OF PHYSICS AND

ASTRONOMY

UNI VERSITY ROCHESTER

ROCHESTER NY 14627

U.S.A.

HELMKEN HENRY F DR

SMITHSONI AN

ASTROPHYSICAL OBS

60 GARDEN ST

CAMBRIDGE MA 02138

U.S.A.

HEMMLEB GERHARD DR

ZENTRALINSTITUT

PHYSIK DER ERDE

TELEGRAFENBERG A 17

DOR - 15 POT SOAM

$G \cdot D \cdot R$.

HENOUX JEAN-CLAUDE DR

OBSERVATOIRE

DE MEUDON

F-92 I9O MEUDON

FRANCE

HENRY RICHARD C PROF.

PHYSICS DEPARTMENT

JOHNS HOPK INS UNIV.

BALTIMORE MD 21218

U.S.A.

HERBST WILLIAM DR

ASTRONOMY DEPT

WESLEYAN UNIV

MIDOLETOWN CT 06457

U.S.A.

HERMAN RENEE DR

7 HIS RUE TRUDON

F-92 160 ANTONY

HERS JAN

6573 SEDGEFIELD

SOUTH AFRICA

HESSER JAMES E DR DOMINION ASTRUPHYS ICAL OBSERVATORY 5071 W SANNICH RD VICTOR I A BC V $8 \times 3 \times 3$ CANADA

HEWITT A V DR

US NAVAL OBSERVATORY

PLAGSTAFF AZ 86001

U.S.A. 
HEY JAMES STANLEY DR 4 SHERTLANDS GLOSE

WILLINCDCY

SUSSEX ENZ2 JJE

L.K.

HIPBS ALBERT $R$ MGR PLANS

JET PRCPULSION

LAPCRATORY

4030 OAK GROVE DRIVE

PASALENA CA 91103

U.S.A.

HICALSC MIGUEL A DR

F ACULTAD DE CIENCIAS

CIULAD UNIVERS ITAR IA

Z $\triangle R A C D Z A$

SPAI:

HICES LLOYPA A DR

CF ASTREPHYS ICS

NAT RES COUNC CANADA

CTTAWA CNT KIA ORG

CANAE?

HILF EBERHARD R H PH. D

PAUL-WAGNERSTR 56

C-6I;) DARMSTADT

GERNANY, F.R.

HILL PHILIP W DR

UNIVERS ITY

CESERVATORY

ST ANOREWS

FIFE KYIG $9 L Z$

U.K.

HILLS JACK G OR

CEPT ASTRIJN ASTROPHY

MICHIGAN STATE UNIV

PHYS-ASTROPHYS BLCG

E LANSING MI 48024

U.S.A.

HINTEREGGER HANS E DR

AIR FCRCE GEOPHYS ICS

HANSCUN AFE MA U1731

U.S.A.

HIFAEAYASHI HISASHI DR

TCKYC ASTRONOMICAL

CESERVATORY

CSAWA MITAKA

TCKYC 181

JAPAN

HIRCSE HIUEO PROF

TCKYC ASTRONOMICAL

CESERVATORY

OSAWA 2-CHOME MITAKA

TCKYC ISI

JAPA?

HITCTSUYANAGI JUICHI PROF

PEPT OF ASTRONOMY

DRANAK I SENDAI 98 ?

JAPAN

HCAG ARTHUR A DR

LCWELL OPSERVATCRY

SCX 1269

FLAGSTAFF AZ 86002

U.S.A.
HEYDEN FRANCIS J SJ DR MANILA OBSERVATORY

$P$ O BOX 1231

MANILA REPUBLIC OF PHILIPPINES

HICKSON PAUL OR

DEPT GEOPHYS ASTRON

UNIVERSITY OF B C
VANCUUVER BC VGT IW5

VANCOUVER
CANADA

HIDE RAYMOND PROF

GEOPHYSICAL FLUID

DYNAM ICS LARORATORY

METEOROLOGICAL OFF

BRACKNELL BERK SHIRE

U.K.

HILDITCH RONALD W DR

UNIVERSITY

ORSERVATORY

BUCHANAN GARDENS

ST ANDREWS KYI6 9LZ

U.K.

HILL GRAHAM DR

DOMINION ASTRO OBS

NAT RES CDUNC CANADA

5071 WEST SAANICH RD

$\checkmark$ ICTORIA BC V $8 \times 3 \times 3$

CANADA

HILLEBRANDT WOLFGANG PH D MPI FUER PHYSIK UND

ASTROPHYSIK

FOEHRINGER RING 6

D-8000 MUENCHEN 40

GERMANY, F.R.

HILLS RICHARD E DR

CAVENOISH LABORATORY

MADINGLEY RD

CAMBR IDGE CB 3. OHE

U.K.

HINTZEN PAUL MICHAEL N DR NASA-GSFC

GREENBELT MD 20771

U.S.A.

HIRAI MASANORI DR

OF EDUCATION

729 MUNAKATA

FUKUOKA $811-41$

JAP AN

HIRST WILLIAM P

1 CL IFFOR D CRE SCENT

BERGVL IET CP

HJALMAR SON AKE G DR

ONSALA SPACE

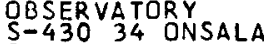

SWEDEN

HOANG BINH DY DR

OQSERVATOIRE

DE MEUDON

F-92190 MEUDON

FRANCE
HEYVAERTS JEAN DR

OB SERVATOIRE

DE MEUDON

F-92190 MEUDON

FRANCE

HIDAJAT BAMBANG PROF DR

BOSSCHA OBSERVATORY

IEMBANG JAVA
INDONES I A

HIEI EI JIRO DR

TOKYO ASTRONOMICAL

OBSERVATORY

OSAWA MITAKA

TOKYO 181

JAPAN

HILONER ERNEST DR

HIGH ALTITUDE

OBSERVATORY

P O BOX 300

BOUDER CD 80307

U.S.A.

HILL HENRY ALLEN DR

BLDG 81

UNIV OF ARIZONA

TUC SON AZ 85721

U.S.A.

HILLIARD R DR

STEWARD OBSERVATORY

UNIV OF ARIZONA

TUC SON AZ 85721

U.S.A.

HILTNER W ALBERT PROF

UEPT VER ASTRONOMY

ANN ARBOR MI 48109

U.S.A.

HIPPELEIN HANS H DR

D-6900 HEI DELBERG

GERMANY, F.R.

HIRAYAMA TADASHI DR

TOKYO ASTRONOMICAL

OBSERVATORY

OSAWA MITAKA

TOKYO 181

JAPAN

HIRTH WOLFGANG ERNST PH D

THEODOR-HEUSSTR 18

D-5354 WE I LERSW I S T

GERMANY, F.R.

HJELLMING ROBERT M DR

NATIONAL RADIO ASTRO

NOMY OBSERVATORY

SOCORRO NM B7801

U. S.A.

HOBBS LEWIS M DR

YERKES OBSERVATORY

UNI VERSITY OF

CHICAGO

WILLIAMS BAY WI53191

U.S.A. 


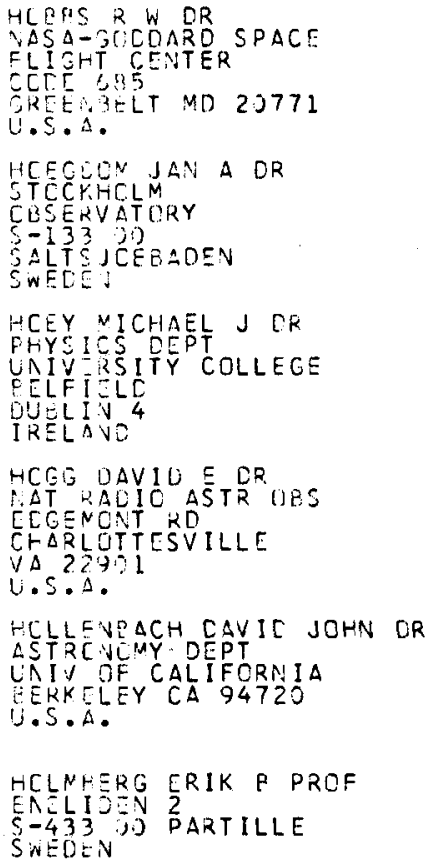

HCLNHERG ERIK P PROF

ENELISEN 2

$S-433$ JO PARTILLE
$S N E D E N$

HENEYCUTT RI KENT PROF ASTRCNCMY DEPT

SWA IA HALL WEST

INEIANA UNIVERSITY

ELCCNINGTON IN 47425

U.S.A.

HCFPE J A PROF CR

CEAN NEFRGSTR

G.L.k.

HCRI SENICHIRO PROF

OEPT OF ASTRONOMY

UAIVERS ITY OF TOKYO

EUAKYU TOKYO 113

JAPAN

HCRSKY Z DR

ASTRCNCNICAL INST

CSAV

GLECCSKA

12123 PRAHA

CZECIIOSLOVAKIA

HCSKIN MICHAEL A CR

CHURCHILL COLLEGE

U.K.

HCSOYANA KENNOSHUKE DR INTERNAT IONAL LAT ITU DE C.SERVATORY HCSHIOACKA 2-CHOME NIZUSAWA IWATE O23 JAPAN

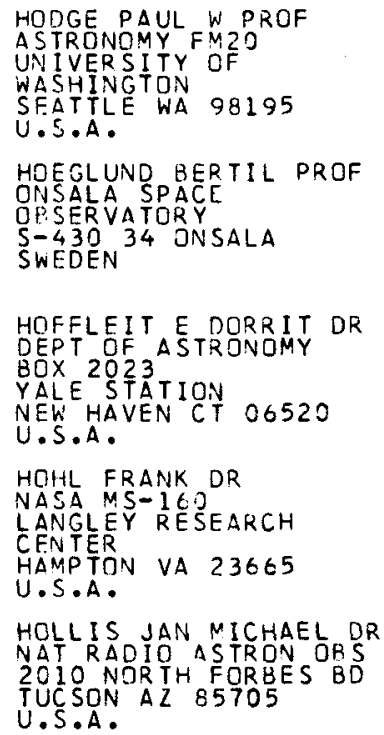

HCNG SEUNG SOD DR

COLLEGE NATURAL SC SEOUL NAT IONAL UNIV SEOUL 150

REPUBLIC OF KOREA

HORAK TOMAS B DR

GEDFYZ IKA NP

JECNA $29 A$

$6 I 200$ BRNO

CZECHOSLOVAKIA

HOROWI IZ GERALD PROF RACAH INST CF PHYS HEBREW UN I VERSITY JERUSALAM ISRAEL

HOR TON BRIAN H OR

DEPT OF PHYSICS

UN IV OF $A D E L A I D E$

ADELAIDE S A $5 \mathrm{CO}$ AUSTRALIA

HOSK ING ROGER J PROF MATHEMATICS DEPT UN IVER S I TY WA I KATO HAMILTON

NEW ZEALAND

HOTINL I METIN DR

UN IVER SITE

RASATHANESI

UN IVER SI TE ISTANBUL
HOEG ERIK LEKTOR COPENHAGEN OBS BRDRFELCE

$D K-434 \%$ TOLLOSE

DENMARK

HOEKSTRA ROEL CR

SPACE RESEARCH

LABORATORY

UTRECHT

THE NETHERLANDS

HOFFMAN JEFFREY ALAN DR

NASA-JSC

HOUSTON TX 77058

U.S.A.

HCLDEN FRANK

$7 O$ CANNCCK ROAD

STAFFORD STI7 DQO

U. K.

HOLLOWAY NIGEL J DR

UNIV OF SUSSEX

FALMER

BRIGHTON BNI $90 \mathrm{OH}$

U.K.

HOLZER THOMAS EDWARD OR HIGH ALTITUDE OBS NCAR PO $80 \times 3000$

BOULDER CO 80307 U.S.A.

HCOGHOUDT $B$ G IR

PRINSENLAAN IO

2341 KT OEGSTGEEST

THE NETHERLANDS

HORAK ZDENEK PROF DR

VIETNAMSKA

16000 PRAHA 6

HOR SKY JAN DR

DEPT OF THEOR PHYS

PURKYNE UNIVERS ITY

KOTLARSKA 2

61137 BRND

CZECHOSLOVAKIA

HOSHI REIUN OR

DEPT OF PHYSICS

NISHIIKEBUKURO $3-\mathrm{CH}$

NOSHIMA TOKYO 171

JAPAN

HOSOKAWA YOSHIMASA H PRDF

DEPT OF PHYSICS

YAMAGATA UNI VERSITY

KOSHIRAKAWA I CHOME
YAMAGATA CITY 990

JAPAN

HOUCK JAMES R

ASTRONOMY DEPT

CORNELL UNIVERSITY

I THACA NY 14853

U. S.A. 


\section{HCLK NANCY OR \\ CEPT OF ASTRCNCMY \\ 1045 PHYS-ASTRO BLDG \\ $A A N$ ARECR MI 48109 \\ U.S.A. \\ HCUTGAST JAKOA CR \\ 3512 UK UTRECHT \\ 3512
THE NETHERLANOS}

\section{HCWARO III WILLIAM E OR \\ CIV ASTRONOMICAL SC \\ NAT SCIENCE FOUNC \\ WDSHINGTCN DC 20550 \\ U.S.A.
HCWSE H DEREK
NAT IC UAL MAR IT IME
NUSEUM GREENWICH
LCACCY SEIS ONF
U. $k$.

HUA CHON IRUNG CR

VILLA LA MAJCRICA

LES EMEUS

- 1319; ALLAUCH

\section{FRANCE}

HUEENET HENRI CR

STERKENWACHT

SCANENHORGH

ZCANENBURS,

UTHECHT 2530

THE NETHERLANDS

HUCHES JCHN PETER DR

CTE FCR ASTROPHYSICS

GU GARCEN STREET

CANERIDGE MA 52136

U.S.A.

HUERNER WALTER F CR

LCE ALAMCS SCIENTI-

FIC LABOEATORY

LCS ALANOS NM 87545

U.S.A.

IUGHES VICTOR A PROF

DEPT GF PHYSICS

GUEENS UNIVERS ITY

CANALA

HUNNER CAVID C TR

JCINT INST F LAECRA-

TCRY ASTRQPHYSICS

FCULCER

HUNPHRIES C M DR

RCYAL CBSERVATORY

PLACKFCIRD HILL

EIIUURGH EHA 3 HJ

U. K.

HUNSIEAD RICHARE W DR

SCHCEL CF PHYS ICS

URVERSITY OF SYUPEY

SYCNEY iN $S$ W 2006

AUSTRALIA

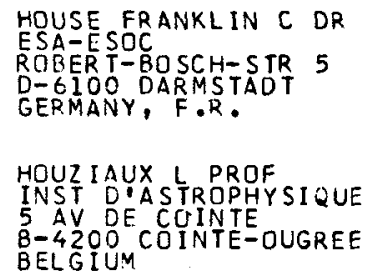

HOWARD ROBERT F DR HALE OBSER VATORIES PASADENA CA 91101 U.S.A.

HOYLE FRED SIR

COCKLEY MOOR

DOCKRAY

PENR ITH

CUMBRIA CALI CLG

U.K.

HUBBARD WILLIAM B PROF

OEPT DF PLANETARY

SCIENCES

UNIVER SITY AR I ZONA

TUCSON AZ 85721

U.S.A.

HUPER MARTIN C DR

ATOM E ASTROPHYSICS

GROUP

ETH ZENTRUM

CH 8092 ZUER ICH

SWITZERLAND

HUCHTMEIER WALTER $X$ DR

MPI FUER

RACIOASTRONOMIE

AUF DEM HUEGEL 69

D- 530 D BONN I

HUGHES DAVID W DR

DEPT OF PHYSICS

THE UN IVER SI TY

U.K.

HUGUEN IN G RICHARD

DEPT PHYS AND ASTRON

UNIV OF MASS

GRADUATE RES CTR

AMHERST MA 01002

U.S.A.

HUMPHREYS CURTIS JUDSON DR

6065 RIVERSIDE AVE

RIVER SIDE CA 92506

U.S.A.

HUNDHAUSEN A DR

HIGH AL T ITUDE OBS S

U.S.A.

HUNT GARRY E DR

LAB PLANETARY ATMOSP

DEPT PIHYS ASTRONOMY

GOWER STREET

LONDON WCIE. GBT

$U \cdot K$.
HOUSE LEWIS L DR

NIGH ALTITUDE OBS

P O BOX 3000

BOULDER CO 80303

U.S.A.

HOVENIER J W DR

DEPT PHYSICS/ASTRON

DE BOELELAAN

LO 1 HV AMSTERDAM

THE NETHERLANDS

HOWARD W MICHAEL OR

LAWRENCE LIVERMORE

LABORA TORY L 71

LIVERMORE CA 94550

U.S.A.

HQYNG PETER DR

SPACE RESEARCH LAB

BENELUXLAAN 21

UTRECHT

THE NETHERLANDS

HUBE DOUGLAS P DR

UNI VERSITY ALBERTA

EDMONTON ALTA T6G2JI

CANADA

HUBERT-DELPLACE

ANNE - MARIE DR

OBSERVATOIRE

DE MEUDON

F-92 190 MEUDON

FRANCE

HUDSON HUGH S DR

PHYSICS C-OII

UC SD

LA JOLLA CA 92093

HUGHES JAMES A DR

US NAVAL OBSERYATORY WASHING TON DC 20390

U.S.A.

HULSAOSCH A N M OR

STERRENKUNDIG INST

KATH UNIVERSITEIT

NI JMEGEN

THE NETHERLANDS

HUMPHREYS ROBERTA M PROF

ASTRONOMY DEPT

UNI VERSITY OF

MINNE SOTA

MINNEAPQLIS MN 55455

U.S.A.

HUNGER KURT PROF

INST THEOR PHY STERN

NEUE UNIV PHYS ZENTR

DLSHAUSENST CB N GIC

D-2 300 KIEL I

HUNT ROLAND DR

DEPT OF MATHEMATICS

UNIV OF STRATHCLYDE

26 RICHMOND STREFT

GLASGOW GI 1 XH

U.K. 
HUNTEN DONALD M PROF LUNAK AND PLANETARY

LAECRATCRY

UNIVERSITY $A R I Z C N A$

TUCSUN AZ 85721

U.S.A.

HURNIK HIERONIM PROF

CESERWATOR IUM

ASTRCNOMICZNE UAM

SLONECLNA 36

$60-2.96$ POZNAN

PCLAND

HUTCHEON RICHARD J DR $X-R A Y$ ASTRONOMY GR

PHYSICS DEPT

LIIV OF LEICESTER

LEICESTER LEI TRH

L.K.

HYLAND A R HARRY DR

CESERVATORY

WCLEN PO ACT 2606

AUSTRALIA

HYUN JCNG-JUNE PROF

SECUL NAT UNIVERSITY

SINLIM-DONG

KWANAK -KU

RECUL 151 IIC KOREA

I PAEIVUCV KHURS ANOKUL DR

INST OF ASTROPHYSICS

$\triangle C A D$ OF SCS DF THE

TADJIK SSR

734673 DUSHANBE

U.S.S.R.

IBEN ICKO JR PROF

$\triangle S T R C$ NOMY DEPT

UNIVERSITY DF

ILLINOIS

URFANA IL 61801

U.S.A.

ICLIS G $M$

INSTITUTE F HISTORY

CF SCI AND TECHNOLD-

10312 MOSCOW

U.S.S.R.

IKEUCHI SATQRU OR

DEPT OF PHYSICS

HEKKAIDO UNIVERS ITY

SAPPCRO OSO

JAPAN

ILL NARTON J OR

CESERVATORY

65 I BAJA

HUNGARY

INRERT MAURICE DR

CESERVATOIRE

CE NARSEILLE

2 PLACE LE VERRIER

$F-13 ! 14$ MARSEILLE

FR $\triangle N C$

IANANEN KIMMO A PROF

CRESS PHYSICS DEPT

4700 KEELE STREET

CCWNSVIEW DNT MIJIP3

CANALA
HUNTER CHR ISTOPHER PROF

MATHEMATICS DEPT

FLOR IDA STATE

UNIVERSITY

TALLAHASSEE FL 32306

U.S.A.

HURUHATA MASAAKI PROF

TOKYO ASTRONOMICAL

OBSERVATORY

OSAWA 2-CHOME

MITAKA TOKYO 181

JAPAN

HUTCHINGS JOHN B DR

DOM INION ASTRO OBS

NAT RES COUNC CANADA

5071 WEST SAAN ICH RO

$\checkmark$ ICTOR IA BC $\vee 8 \times 3 \times 3$

CANADA

HYNEK J ALLEN PROF

DEARBORN OB SER VATORY

NORTHWE STERN

UN IVERSITY

EVANSTON IL 60201

U.S.A.

IANNA PHILIP A

PO BOX 3818

CHARLOT TE SVILLE

VA 22903

U.S.A.

IBANOGLU C DR

OBSER VATORY

BDRNOVA IZMIR

TURKEY

ICHIMARU SETSUO DR DEPT OF PHYSICS

UNIVERSITY ÖF TOKYO

BUNKYO-KU

TOKYO 113

JAP AN

I J IMA SHIGETAKA PROF

TOKYO ASTRONOMICAL

OBSERVATORY

OSAWA MITAKA TOK 181

JAPAN

IKHSANOV ROAERT $N$ DR

PULKOVO OBSERVATORY

196140 LENINGRAD

U.S.S.K.

ILLES ALMAR ERZSEBET DR KONKOLY OB SERVATORY

$\mathrm{HOX} 67$

1525 BUDAPEST

HUNGARY

IMSHENN IK V S DR

INST TIEOR EXP PHYS

117259 MOSCOW

U.S.S.R.

INOUE TAKESHI PROF

KYOTO SANGYO

UNIVER SITY

KYOTOO 603

JAPAN
HUNTER JAMES H
5641 DR
APT PRAOO DR

TAMPA FL 33617

U.S.A.

HURUKAWA KIICHIRO DR

TOKYO ASTRONOMICAL

OBSERVATORY

OSAWA MITAKA

I II TOKYO

JAPAN

HYDER C L DR

NASA SOLAR GROUP

UNIV OF NEW MEXICO

800 YALE BLVD N E

ALBUQUERQUE NM 87106

U. S.A.

HYSOM E J.

HARPALNEEN

HERTS AL5 5PP

U. K.

I ANNINI GUALBERTO DR OBSERVATORIO ASTRONO MICO DE CORDOBA

$\angle A P R I D A \quad 854$

5 OOO CORDOBA

ARGENTINA

IBBETSON PETER AARON DR

WISE OBSERVATORY

TEL AVIV UNIVERSITY

TEL AVIV

I SRAEL

ICKE $V$ DR

DEPT OF ASTRONOMY

UNI V OF MINNESOTA

$116 \mathrm{CHURCH}$ ST $S$ E

MINNEAPOLIS MN 55455

U.S.A.

IKEDA TETSURO DR

1 - 18 TENMONDAI DORI MI ZUSAWA IWATE O23 JAPAN

I KHSANOVA VERA N DR PULKOVD OBSERVATORY 196140 LENINGRAD

U.S.S.R.

ILLINGWORTH GARTH D DR P PEAK NAT OBS

TUC SON AZ 85726

U.S.A.

I NGRAO HECTOR C

CAMBRIDGE SYST CORP

545 TECHNOL

CAMBRIDGE MA 02139

U.S.A.

I OANNI SIANI $B$ K DR

MAIN ASTRONDMICAL

OBSERVATORY PULKOVO

196140 LENINGRAD

U. S. S.R. 
ICSHPA B A DR

IASTITUTE OF T ERRE-

ICNCSPTER

142032 AKADDEMGORCIDOK

U.S.S.R.

IRIARTE E MR

I:FT Y ELECTRONICA

APT FOSTALES 21651

PUEELA PUE

ISAAK GEORGE R CR

CSPT GF PHYSICS

UNIV OF 3 I RMINGHAM

EIRNINGHAM BI5 2TT

ISCRE SHUZO DR

TCKYO ASTRONOMICAL

CESERVATORY

IIII NITAKA TOKYO

JAPAN

ISSERSTEDT JOERC CR
INSTITTUT ASTRONOMIE

UNE ASTROPHYSIK

AN HUBLAND

C-780) WU ERZBURG

GERMANY, F.R.

IV ANCHUK VICTOR I DR

KIEV UNIVERSITY

$252053 \mathrm{KIEV}$

U.S.S.R.

I V SNCV -KHOLODNY G S DR

INSTITUTE OF APPLIED

GECPHYS ICS USSR

ID 7150 MOSCOW

U.S.S.R.

IWANCWSKA WILHELMINA OR

CHCPINA 1218

E $7-126$

PCLANO

JA $\triangle K K D L A$ TQIVOS

CESERVATORY

TAHTITCRNINMAK I

SF-OCI 130 HELS INK I

FINLANC

JACKSON C MR

CRS AUSTRAL YALE -

CCLUNC 263

SAN JUAN

JACKSON PETER COUGLAS DR ASTRCNCMY PROGRAM

UAIV OF MARYLAND

CCLLEGE PK MD 20742

JACCPSEN THEODOR S PROF

6205 I TTH AVE NE

U.S.A.
IPSER JAMES R PROF

933 E 56TH ST

CHICAGO IL 60637

U.S.A.

IRVINE WILLIAM M PROF

ASTRONOMY GRC

TOWER B

AMHERST MA OIOO3

U.S.A.

ISHIDA KEIICHI OR

TOKYD ASTRONOMICAL

OBSER VATORY

2-21-1 DSAWA MITAKA

JAPAN

I SRAEL WERNER PROF

UNYSICS DEPT

ALBERTA

EDMONTON ALTA TGGZEL

CANADA

ITO KENSUKE A PROF

R IKKYO UN I VERS ITY

NISHI-IKEBUKURO

TOKYO 171

JAPAN

IVANOV $G$ R DR

DEPT DF ASTRONQMY

ANTON IVANOV $\$ T 5$

P 0120

1126 SOFIA

BULGAR IA

IVES JOHN CH OR MULLARD SPACE SC LAB UNIV COLLEGE LONOON LONDON

U.K.

IWASAKI KYOSUKE DR KWASAN OBSER VA TORY YAMASHINA KYOTO 607 JAPAN

JACCHIA LUIGI G DR

CENTER FOR

6 OARDEN

CAMBRIDGE MA 02138

U.S.A.

JACKSON J C DR

16 THE PARK

NEWARK

NOTTINHAMS NG24 ISO

U.K.

JACKSON WILLIAM M DR

DEPT OF CHEMISTRY HOWARD UNI VERSITY

WASHING

JACQUINQT P DR

$\angle A B$ AIME COTTON

BAT 505

UNIVERSITE PARIS SUD

F-91405 DR SAY CEDEX
I RELAND JOHN G DR

C-O 13 GORDON RD

BELVEDERE

KENT DAIT GEA

U.K.

IRWIN JOHN B PROF

2744 TYNDALL AVE

TUCSON AZ B5719

U.S.A.

ISHI ZAWA TOSHISUKE A PROF DEPT OF ASTRONOMY UNIVERSITY OF KYOTO KYOTO 606

JAPAN

I SSA ALI DR

HE LWAN OBSERVATORY HELWAN-CAIRO

ARAB REP. OF EGYPT

I TOH NAOKI DR

SOPHIA UNIVERSITY

7 KIOICHO CHIYODA-KU

TOKYO 102

JAPAN

I VANOV VSEVOLOD $V$ OR

ASTRDNOMICAL OBS

IOTH LINIJA 3

I99178 LENINGRAD

U.S.S.R.

I WANI SZEWSKA CECYLIA DR

INST OF ASTRONOMY

N COPERNICUS UNIV

CHOP INA I 18

P7-1OO

I ZVEKOV $V A$ AR

INST OF THEORET ICAL

IO KUTUZOV QUAY

192187 . LENINGRAD

U.S.S.R.

JACKISCH G DR

ZENTRALINSTITUT FUER

ASTROPHYSIK

STERNWARTE SONNEBERG

DDR-64 SONNEBERG

$G \cdot D \cdot R$.

JACKSON PAUL DR

INST FUER ASTRONOMY

DER UNIV WIEN

TUERKENSCHANZSTR 17 $A-1180$ WIEN

JACOBS KENNETH C DR

CENTER 344

BELL LABORATORIES

600 MOUNTAIN AVENUE

MURRAY HILL NJ 07974

U.S.A.

JAEGER FRIEDRICH W PROF

ZENTRALINSTI TUT FUER

SOLARTERR PHYSIK

DOR-I 5 POTSDAM

G.D.R. 
JAFFE WALTER JOSEPH DR NAT RAOID ASTRONOMY

CBSERVATORY

EEGENONT ROAD

CHARLOTTESVL VA22901

U.S.A.

JANAR CLAUDE A J CR

\& RUE DE BATTY

B-420O COINTE-DUGREE

SELGIUM

JAMESON RICHARD F DR ASTRCNCMY DEPT

LEICESTER UNIVERSITY

LEICESTER LEI 7RH

U.K.

JANKOVICS ISTVAN CR KCAKCLY OBSERVATURY CCX 67

I 255 BUDAPEST

HUNG $\triangle R Y$

JARZEHCWSKI TADEUSZ TIR

ASTRCIVOMI.CAL

INST ITUTE

KCPER IKA II

$51-62 ?$ WROCLAW

JASTROW ROBERT

INST F SPACE STUCIES

2880 ZROADWAY

NEW YORK NY 10025

U.S.A.

JAYARAJAN A P MR

ASTRCPHYSICAL OBS

$K C$ CAIKANAL 3

INCIA

JEFFREYS HAROLD PRDF SIR

IGI HUNT INGOON RCAC

U.K.

JENKINS EDWARD B DR

CBSERVATORY

PRINCETCN NJ 08540

U.S.A.

JENNER DAVID C PRDF

DEPT OF ASTRONOMY

UNIV.CF WASHINGTON

SEATTLE, WA 98195

U.S.A.

JENSCH A

PESTALCZ2ISTR

CLR 6 . C. R.

JCCKERS KLAUS DR NPI FUER AERONOM IE PCSTFACH 25

$0-3411$ KATLENBURG-L

GERNAVY, F.R.
JAHREISS HARTMUT DR

ASTRONOMI SCHES

RECHEN INSTITUT

MOE ENCHHOF STR $12-14$

D-6900 HE IDELBERG I

GERMANY, F.R.

JAMES JOHN F MK

SCHUSTER LABORATORY

THE UN IVER SITY

MANCHESTER MI3 SPL

U.K.

JANES KENNETH A DR

ASTRONOMY DEPT

BOSTON UNIVERSITY

725 COMMONWEAL TH AVE

BOSTON MA O2215

U.S.A.

JARNEFELT GUSTAF J PROF

OHJAAJANTIE 3 B 18

FINLAND

JASCHEK CARLOS O R PROF DBSERVATOIR

11 RE L UN I VER SITE

F- 67000 STRA SBOURG

FRANCE

JAUNCEY DAVID L DR

RADIOPHYSICS

$P O B O X 76$

EPPING NSW 2121

AUSTRALIA

JEFFER IES JOHN T DR

INST FOR ASTRONOMY

HONDLULU HI 96822

U.S.A.

JEFFREYS WILLIAM H DR

ASTRONOMY DEPT

UNIVERSITY OF TEXAS

AUSTIN TX 78712

U.S.A.

JENK INS L F MS

YALE UN IV OBS

$B \cap X 2023$

YALE STATION

NEW HAVEN CT 06520

U.S.A.

JENN INGS R E DR

DEPT PHYSICS AND AST

UNIVERSITY COLLEGE

GOWER STREET

LONDON WCIE $6 B T$

U.K.

JENSEN ERERHART PROF

INSTITUTE OF THEORE-

YICAL ASTROPHYSICS

UN IVERSITY OF OSLO

BLINEERN OSLO 3

NDRWAY

JOHANSEN KAREN T LEKTOR I GEL SO

DK -4300 HOLBAEK

DENMARK
JAKI MIEC JERZY DR ASTRONOMICAL INST KOPERNIKA I 51622 WROCLAW POLLAND

JAMES RICHARD A DR

DEPT OF ASTRONOMY

THE UNIVERSITY

MANCHESTER MIS $9 P L$

U. K.

JANICZEK PAUL M DR US NAVAL OBSERVATORY WASHING TON DC 20390 U. S. A.

JARRETT ALAN H PROF BOYDEN OBSERVATORY $P O B O X 334$ RLOEMFONTEIN 9300 SOUTH AFRICA

JASCHEK MERCEDES DR II R DE L UNIVERSITE F-67000 STRASBOURG

FRANCE

JAVET PIERRE PROF AV DE BEAUMONT 36 CH-1012 LAUSANNE SWI TZERLAND

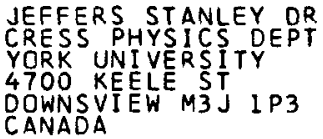

JELLEY JOHN Y PHD FRAS NP-DIV BLDG 8 AERE U.K.

JENKNER HELMUT OR UNIV-STERNWARTE TUERKENSCHANZSTR 17 $A-1180$ WIEN AUSTRIA

JENNISON ROGER C PROF

ELECTRONICS LAE

UNI VERSITY OF KENT

CANTERBURY CT2 7NT

U. K.

JERZYKIEWICZ MIKOLAJ DR ASTRONOMICAL INST KOPERNIKA I

$51-622$ WROCLAW

JOHNSON DONALD R DR 716 TIFFANY CDURT GAI THER SBURG MD20760 U.S.A. 


\section{JCHRSON FRED M PROF CR DEPT PIIYS AND ASTRON CALIF STATE UNIV 230ह $=$ AMERIGE AVE FULLERTCN CA 92634 U.S.A. \\ JCHNSON MARTIN ER PHYS ICS DEPT \\ UNIVERSITY \\ EIRNINGHAM B15 2TT \\ $U . K$.}

JCKIPII J R PROF LEPT OF PLANETARY SC ARIZCNA

TUCSEN AZ 95721

U.S. A.

JCNES RERNARD J T OR

IAST CF ASTRONOM

CANERIDGE

U.K.

JCVES ERIC $M$

LCS ALIMOS SCIENTI-

FIC LARORATORY

LCS ALAMOS NM 87545

U.S. 4 .

JCNES JAMES DR

UNIV WESTERN ONTARIO

LCNCEV ONT NGA 5 EG

CANACA

JCRDAN CAROLE DR

LEPT THEORET ICAL PHY

I KEPLE ROAD

CXFCKD UNIVERSITY

CXFERD CXI BNP

$\mathrm{U} \cdot \mathrm{K}$.

JCRGENSEN HENIVING E LEKT

UAIV RTSITY

CESERVATORY

CIK-135j COPENHAGEN K DENMARK

JESHI NOHAN N LR

RALIC ASTRONOMY

CENTKE CF TIFR

PEST $80 \mathrm{X}^{8}$

CETACAMUND 643001

I $A[I A$

JUGAKU JUN OR

TCKYO ASTRONOMICAL

LESERVATORY

CSAWA MITAKA

TCKYO 181

JAPA:

JUPP ALAN A DR

URIVERSITY LIVERPOOL

$P$ C FXX 147

LIVERPCOL L69 $3 B X$

U.K.

KAQURAKI MASAKI PROF

4-24-9 KICHIJYOJI

MINAMI

NUSASHINO TOKYO 180

$J \triangle P A N$
JOHINSON HOLLIS R PROF ASTRONDMY DEPT

INDIANA UNIVER SITY

BLOOMINGTON IN 47405

U.S.A.

JOHNSON TORRENCE $V$ DR JET PROPULSION

LABOR A TOR Y

4800 OAK GROVE DRIVE

PASADENA CA 91103

U.S.A.

JOLY FRANCOIS OR

UN IVER S I TE DE

BORDEAUX

40 RUE LAMARTINE

F- 33400 TALENCE

FRANCE

JONES BUR TON DR

LICK OBSERVATORY

UN IVER SITY

OF CAL IFORNIA

SANTA CRUZ CA $\$ 5064$

U.S.A.

JONES FRANK CULVER DR

NASA-GSFC

CODE 660

GREENBELT MD 20771

U.S.A.

JUNES JANET E DR

MAD INGLEY ROAD

CAMBR IDGE

U.K.

JORDAN H L OR DIREKTOR

INST PLASMAPHYSIK

KERNFOR SCHUNG SANLAGE

JUEL ICH GMBH PF 365

D-5170 JUELICH 1

JUSEPH J H OR

DEPT OF GEOPHYSICS

AND PLANETARY SC

RAMAT AVIV

I SRAEL

JOSHI SURESH CHANDRA DR UP STATE DBSERVATORY

MANORA PEAK

NAINI TAL 263129

INDIA

JUNG JEAN DR

AMBASS FRANCE WASH

FRENCH SC MISSION

2011 EYE STREET NW

WASHINGTON DC 20006

U.S.A.

JURA MICHAEL DR

DEPT OF ASTRONOMY

UCLS ANGELES CA 90024

U.S.A.

KABURAK I OSAMU DR ASTRONOM ICAL INST SENDAI 980 JAPAN
JOHNSON HUGH M DR

DRGN 32 H 12 BLDG 202

PALO ALTO CA 94304

U.S.A.

JOHNSTON KENNETH

CODE 7134

NAVAL RESEARCH

LABORATORY

WASHINGTON DC 20375

U.S.A.

JONES ALBERT F MR

31 RANUI ROAD

STOKE

NELSON

NEW ZEALAND

JONES DEREK H P DR

ROYAL GREENWICH

OBSERVATORY

HAI L SHAM

EAST SUSSEX BN27 IRP

U.K.

JONES HARRISON PRICE DR

KITT PEAK NAT ORS

SOLAR STATIDN

900 N CHERRY AVE

TUCSON AZ 85726

U.S.A.

JONES THOMAS WALTER DR

DEPT OF ASTRONOMY

UNIV OF MINNESOTA

MI NNEAPOLIS MN 55455

U.S.A.

JORDAN STUART D DR

LAB ASTRO SOLAR PHYS

NASA-GODDARD SPACE

FLIGHT CTR CODE 682

GREENBELT MD 20771

U.S.A.

JOSEPH ROBERT D DR

BLACKETT LAB

AS TRONOMY GROUP

I MPER IAL CDLLECE

LONDON

U. K.

JOSS PAUL CHRISTDPHER DR RIT́M 6-203

CAMBRIDGE MA 02139

U.S.A.

JUNKES JOSEF DR

12 HOHENBRUNNERSTR

D-8014 NEUBIBERG

GERMANY, F.R.

JURKEVICH IGOR DR

3130 PORT WAY

ANNAPOLIS MO 21403

U.S.A.

KADLA ZDENKA I DR

PULKOVO OBSERVATORY

196140 LENINGRAD

U.S.S.R. 


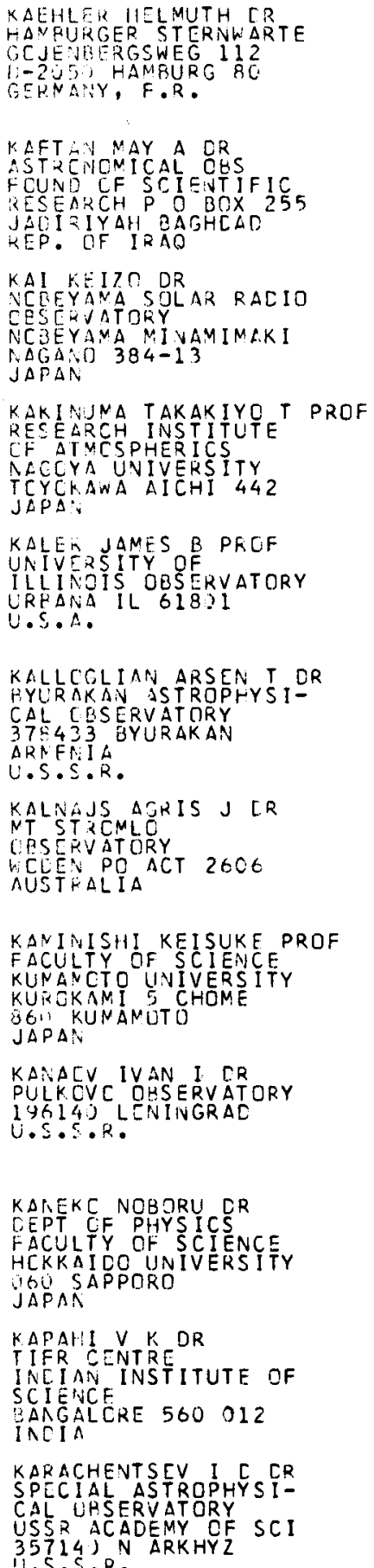

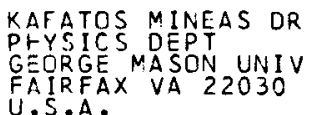

U.S.A.

KAHLER STEPHEN W DR AMER ICAN SCIENCE AND ENG INEFR ING INC TS AV CAMBR JDGE MA O2139 U.S.A.

KAIFU NORIO DR

TOKYO ASTRONOMICAL

OPSERVATORY

OSAWA M ITAKA

TOKYO 181

J APAN KAKUTA CHUICHI DR DE OBSERVATORY MISUSAWA IWATE O23 JAPAN

KALINKOV MARIN PUR DEPT OF ASTRONOMY BULGARIAN AC OF SC IOOO SOF IA

BULGARIA

KALMAN BELA DR HEL IOPHYSICAL OBS $P$ G BCX 30

4010 DEBRECEN

HUNGARY

KAMEL OSMAN M DR FACULTY OF SCIENCES ASTRONOMY DEP CAIRO UNIVERSITY GIZA

ARAB REP. OF EGYPT

KAMP LUCAS WILLEM DR

DEPT ŌF A STRONOMYY

BOSTOH UNIV

725 COMMONWEALTH AVE QOSTON

KAVDEL ROBERT S DR OESERVATOIRE

DE MEUDON

F-92I 95 MEUDON

FRANCE

KANNO MITSUO DR

HICA OESERVATORY

KAM I TAKARA

GIFU-KEN 50613

KAPLAN J DR

DEPT DF PHYSICS

UCLS ANGELES CA 90024

U.S.A.

KARANDIKAR $R \quad V$ PROF

DEPT OF ASTRONOMY

OSMANIA UNIVER SITY

HYDERABAD 500007

INDIA
KAFKA PETER

MPI FUER PHYSIK UND ASTROPHYSIK 8000 MUENCHEN 40

GERMANY, F.R.

KAHN FRANZ D PROF

DEPT OF ASTRONOM NANCHESTER MI 3 P PL

U.K.

KAISER T R PROF

DEPT DF PHYSICS

THE UNIVERSITY

SHEFFIELD S3 TRH

U.K.

KALANDADZE N B DR $\triangle B A S T U M A N Y$ ASTROPHYSICAL OBSERVATORY 383762 ABASTUMANY

U.S.S.R.

KALKOFEN W DR

SMITHSONI AN ASTROPHY SICAL OBSERVATORY GO GARDEN ST

CAMBRIDGE MA 02138 U.S.A.

KALMYKOV A M DR ASTRONOMICAL INSTITU TE UZBEKIAN ACADEMY OF SCIENCES 700700 TASHKENT U.S.S.R.

KAMI JO FUMIO PROF DR DEPT OF ASTRONOMY UNIVERSITY DF TOKYO YAYOI BUNKYO KU TOKYO 113 JAPAN

KAMPER KARLW OR

DAVID DUNLAP

DASER VATORY

RICHMOND HI LL

ONT L4C 4 Y6

CANADA

KANE SHARAD R DR SPACE SCIENCES LAB FORNI A-BERKELEY BERKELEY CA 94720 U.S.A.

KANYO SANDOR DR KONKOLY OBSERVATORY $\mathrm{BOX} 67$ 1525 BUDAPEST HUNGARY

KAPLAN L MR JPL DO OAK GROVE DRIVE PASADENA CA 91103 U.S.A.

KARDASHEV $N S$ DR

SPACE RE SEARCH

INSTI TUTE

USSR ACADEMY OF -SCI

$117810 \mathrm{M}$ 


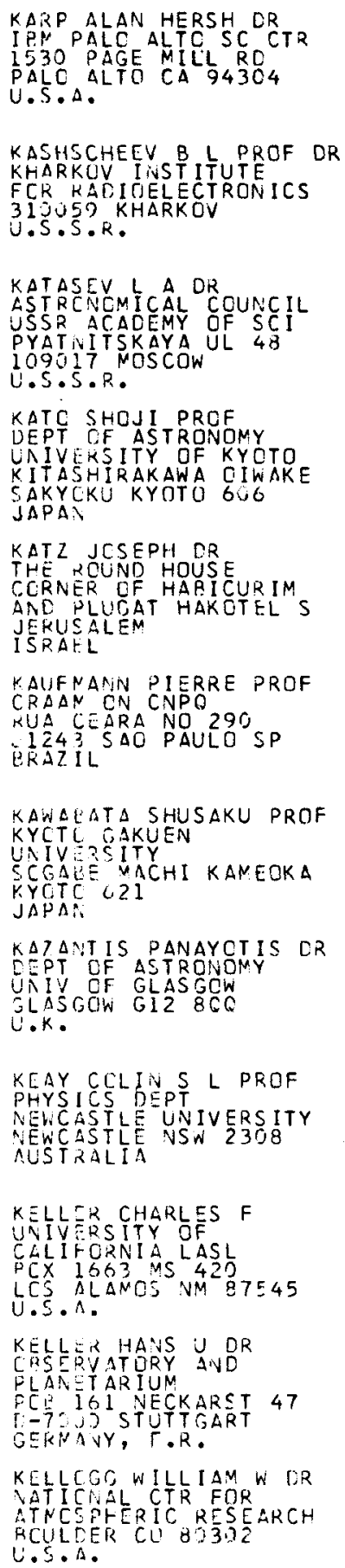

KARP ALAN HERSH OR

I PH PALC ALTC SC CTR

PALC ALTO CA 94304

U.S.A.

KASHSCHEEV $B$ L PROF DR

KHARKOV I ISST ITUTE

FCR KACIOJELECTRONICS

310059 KHARKOV

U.S.S.R.

KATASEV L A OR

ASTRENCMICAL COUNCIL

USSR ACALEMY OF SCI

109017 MOSCOW

U.S.S.R.

KATC SHOJI PROF

DEPT CF ASTRONOMY

UAIVEKS ITY OF KYOTO

KITASHIRAKAWA CIWAKE

SAKYCKU KYOTO 606

JAPAN

KATZ JCSEPH ER

THE RCUND HOUSE

CCRNER OF HARICURIM

ANC PLUCAT HAKCTELS

JERUSALEM

K.AUFNANIN PIERRE PROF

CRAAN CN CNPO

KUA CEARA NO 290

$12435 A 0$ PAULOSP

ERAZIL

KAWEEATA SHUSAKU PROF

KYCTL GAKUEN

UIVAZS ITY

KYCTC O2I

JAPAS

KAT ANTIS PANAYCTIS CR

CEPT CF ASTRQNOMY

UAIV OF GLASGCW

GLASGCW GIL BCQ

U.K.

KEAY COLIA S L PROF

NEWCASTLE UNIVERS ITY

NEWCASTLE NSW 2308

AUSTRALIA

$K \equiv L L E R$ CHARLES F

UNIVTRSITY OF

CALIFORNIA LASL

PCX $16 G 3$ MS 420
LCS ALAMOS NM 87545

U.S.A.

KELLER HAVS U DR

$P L A N=T A R I U M$

FC I 1 NECKARST 47

T:T-JS STUTTGART

GEKWAVY, F.R.

KELLCGS WILLIAM W OR

VATICNAL CTR FOR

ATNCSPHERIC RESEARCH

RCULLER

U.S.A.

KARP INSK I J VADIM N DR PULKOVO OBSERVATORY 196140 LENINGRAO

U.S.S.R.

KASPER U DR

ZNTR INST ASTROPHYSIK

STERNW BABELSBERG

R LUXEMBURG STR ITA

$O D R-150$

KATGERT PETER DR

STERREWACHT

POSTBUS 9513

2300 RA LEIDEN

THE NETHERLANDS

KATO TAKAKO DR

INST OF PLASMA PHYS

NAGOYA UNIVERSITY

RURO-CHO CHIKUSA-KU

NAGOYA 464

JAPAN

KAUFMAN MICHELE DR PHYSICS DEPT

OHID ST UNIVERSITY

174 W $18 T H$ AVE

COLUMEUS OH 43210

U.S.A.

KAULA WILLIAM M PROF

INST OF GEDPHYSICS

UN IVER SI TY

OF CALIFORNIA

LCS ANGELES CA 90024 U.S.A.

KAWAGUCHI ICHIRO PROF

DEPT OF ASTRONOMY

UACULTY OF SCIENCE

606 KYOTO

JAPAN

KAZES ILYA DR

OESERVATOIRE

DE MEUDON

F-92190 MEUDON

FRANCE

KEENAN PHILIP C PROF EMER PERKINS OBSERVATORY

BOX 449

DELAWARE OH 43015

U.S.A.

KELLER GEOFFREY

4ID HIGHGATE A VE

WORTHINGTON OH 43085

U.S.A.

KELLERMANN KENNETH I DR NATIONAL RADIO ASTRO NOMY OBSERVATORY

P O BUX 2

GREEN BANK WV 24944

U.S.A.

KENDERDINE SIDNEY DR MULLARD RADIC ASTRDNOMY LABORATORY

MAD INGLEY ROAD

CAMBR IOGE CB 3 OHE

U.K.
KARYGINA ZOYA Y DR

ASTROPHYSICAL INST

U.S.S.R.

KASTLER ALFRED PROF

SUPERI NURE

45 RUE D ULM
F 75005 PARIS

FRANCE

KATGERT-MERKELIJN J K DR

STERREWACHT

HUYGENS LAB

WASSENAARSEWEG 78

$2300^{\circ}$ RA LEIDEN

THE NETHERLANDS

KATSIS DEM DR

12 RUE VAR

A THENS

KAUFMANN JENS PETER DR

INST F ASTROPHYSIK

T

ERNST REUTER PLATZ 7

D-IDOO BERLIN 10

GERMANY, F.R.

KAWABATA KINAKI PROF

DEPT OF PHYSICS

NAGOYA UNI YERS I TY

FUROCHO CHIKUSAKU

NAGOYA 464

JAPAN

KAWATA YOSHIYUKI DR

KANA ZAWA INSTITUTE

OF TECHNOLOGY

NONOICHO

KANAZAWA MINAMI 921

JAPAN

KAZI MIRCHAK-POLONSKAYA

I DR I TUTE OF THEORE-

I I 12187 LENINGRAD

U.S.S.R.

KEGEL WI LHELM H PROF

INST THEOR PHYSIK

UNIV FRANKFURT

$R-M A Y E R-S T R$ 8 -10
$D-6000$ FRANKFURT/M 1

GERMANY, F.R.

KELLER H UWE DR

MPI FUER AERONOMIE

D-3411 KATLENBURG

GINDAU ${ }^{3}$ G.R.

KELLOGG EDWIN M DR

WATERTOWN MA O2172

WATERT

KENNEDY JOHN E PROF

UNIV OF SASKATCHEWAN

236 ARTS BLDG

SASKATOON

SASKATCHEWAN S7N OWO

CANADA 
KERES KH P PROF DR IAPHA ESTENIAN ACADE MY CF SCIENCES

TCRAVERE

262444 TARTU

U.S.S.R.

KESSLER KARL G DR CTK ABS PHYS QUANT IT NAT GUREAU STANDARDS B- 164 PHYS ICS BLOG WASHIINGTON DC 20234

U.S.A.

KHARADZE E K PROF $\triangle P A S T U M A N Y$ ASTREPHY-

SICAL CBSERVATORY

383762 ABASTUMANY

GECRGIA

U.S.S.R.

KHARITCNOV ANDREJ $V$ DR

ASTROPHYSICAL INST

480008 ALMA-ATA

U.S.S.R.

KFCLCPCV P N DR

TRCNOMICAL INST ASE

117234 MOSCOW

U.S.S.R.

KIANG TAO PROF

DUNS INK OBSERVATORY

CASTLEKNOCK

CC DUGLIN

IRELAND

KIKUCHI SADAEMCN PROF

AST KCIVOMICAL OBS

TCHCKU UNIVERSITY

$A R A M A K$

SENDAI 989

JAPAC

KILKENNY DAVIC CR

UNIV DRSERVATORY

BUCHANAN GAROENS

FIF KNOKIG $9 L 2$

U.K.

KING $\triangle N D R E W$ R DR

ASTRT VCMY UEPT

UNIV UF LEICESTER

LEICESTER

KING IVAN R PRCF

ASTRCNCMY DEPT

UNIV RS ITY OF

CALIFORNIA

EERKELEY CA 94720

U. $\triangle$ A.

KING-HELE DESMONC G MR TCYAL AIRCRAFT EST
FARNEORCUGH HANTS

U.K.

KINOSHITA HIROSHI DR

TCKYO ASTRONOMICAL

CESEKVATORY

CSAWA NITAKA

181 TOKYO

JAPA
KERR FRANK J DR

UN IVER SI TY MAR YLAND

COLLEGE PK MD 20742

U.S.A.

KESTEVEN MICHAEL J L DR

QUEEN S UNI VERSITY

KINGSTON DNT KTL 3NG

CANADA

KHARE BISHUN N DR

306 SPACE SC BLDG

CURNELL UN IVER SITY

ITHACA NY 14853

$U \cdot S \cdot A$.

KHETSUR IANI T S DR

ABASTUMANY ASTROPHY-

SICAL OB SERVATORY

$U . S \cdot S \cdot R$.

KHOLSHEVNIKOV K V OR

VENINGRAD STATE UNI-

OBSER VATORY

IS9178 LEN INGRAD

U.S.S.R.

KIASATPODR AHMAD PRDF

PHYSICS DEPT

SITY O

ISFAHAN

DANESHGAH E ISFAHAN IRAN

KILADZE R 1 DR

ABASTUMANI ASTROPHY-

CAL OBSERVATORY

383762 ABASTUMANY

U.S.S.R.

KIM YONG HEK OR

ACADEMY OF SCIENCES

PYONGYANG ASTRONOMI -

CAL OBSERVATORY

PYONGYANG

DEM PEOPLE'S REP OF KOREA

KING DAVID S PROF

DEPT OF PHYSICS AND

ASTRONOMY

UNIVERSITY N MEXICO

ALBUQUERQUE NM 87131

U.S.A.

K ING R R DR

MEDOCIND CA 95460

$U \cdot S \cdot A$.

KINGSTON ARTHUR E DR

DEPT OF APPLIED MATH

ANE THEORET PHYSICS

QUEEN 'S UN I VER SI TY

BELFAST RTT INN

U.K.

KIPPENHAHN RUDOLF PROF

MPI FUER PHYSIK UND

ASTROPHYSIK

$K$ SCHWARZ SCHILD ST

D-8046 GARCHING $8 \mathrm{M}$

GERMANY, F.R .
KERR ROY $P$ PROF

UNIV OF CANTERBURY

PRIVATE BAG

CHRI STCHURCH

NEW ZEALAND

KHACHIKIAN E Y DR BYURAKAN AS TROPHYSI CAL OBSERVATORY

378433 ARMENIA

U.S.S.R.

KHARIN A S DR

MAIN ASTRON OBS

UKRAINIAN ACAD OF SC

GOLOSEEVO

$252127 \mathrm{KIEV}$

U.S.S.R.

KHOKHLOVA $V$ L DR

ASTRONOMICAL COUNCIL

USSR ACADEMY OF SCI

PYATNITSKAYA UL 48

109017 MOSCOW

U.S.S.R.

KHROMOV G S DR

ASTRONOMICAL COUNCIL

USSR ACADEMY OF SCI

PYATNITSKAYA UL 48

109017 MOSCOW

U. S.S.R.

KIBBLEWHITE EDWARD J DR

INSTITUTE OF

ASTRONOMY

MADINGLEY RD

CAMBRIDGE CB3 OHE

U.K.

KILAR BOGDAN DR

FACULTY OF GEODESY

JUMBL JANA UNIVERSITY

61000 LJUB L JANA

YUGOSLÄVIA

KIMURA HIROSHI DR

PURPLE MOUNTAIN OBS

$\triangle C A D E M I A$ SINICA

NANKING SEPE'S REPUBLIC CHINA

KING HENRY $C$ DR

MCLAUGHLIN PLANETAR

ROYAL ONTARIO MUSEUM

100 QUEENS PARK

TORONTO ONT MSS $2 \mathrm{CG}$

CANADA

KING ROBERT WILSON JR OR

MIT 54-627

CAMBRIDGE MA 02139

U.S.A.

KINMAN THOMAS D DR

KITT PEAK NATIDNAL

OBSERVATORY

POBOX 26732

U.S.A.

KIPPER AKSEL PROF

I APHA

ESTONIAN ACAD OF SC

TORAVERE

20244 TARTU

U.S.S.R. 


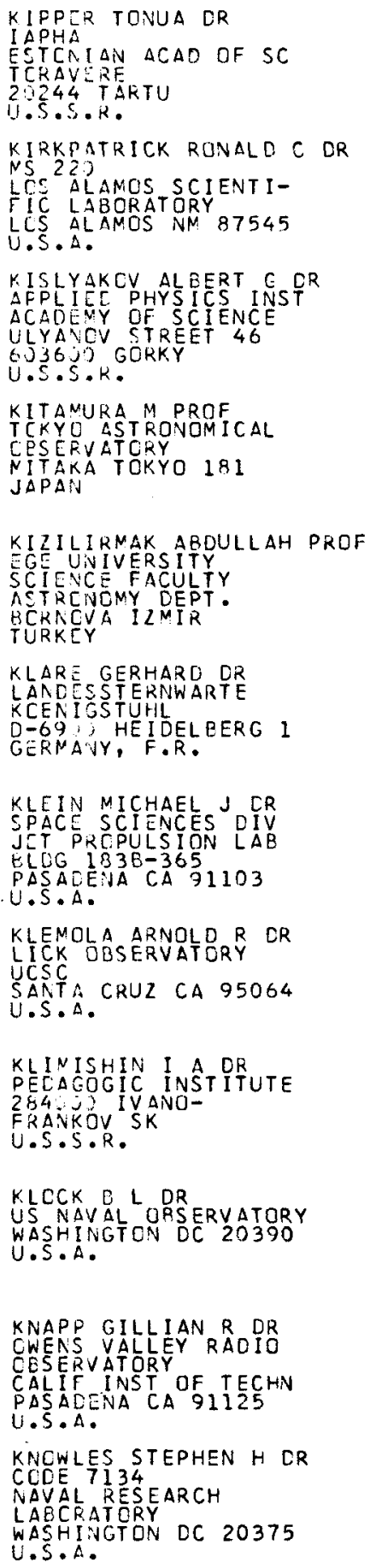

KIRAL ADNAN PROF

UN IV OBSERVATORY

BEYAZIT

ISTANEUL

KIRSHNER RQBERT PAUL DR

DEPT OF ASTRONOMY

UN IV DF MICHIGAN

ANN ARBOR MI 48103

U.S.A.

KISLYUK $V S D R$

MAIN ASTRON OBS

UKRAINIAN ACAD OF SE

GOLOSEEVO

$252127 \mathrm{KIEV}$

U.S.S.R.

KITAMURA SEI ICHI DR

SHIGA UN IVER SI TY

JAPAN

KJAERGAARD PER DR COPENHAGEN UNIVERSI TY OBSERVATORY

OSTER VOLOGADE ${ }^{3}$

DENMARK

KLARMANN JOSEPH PROF

DEPT OF PHYSICS

WASHINGTON

UN IVER S ITY

ST LOUIS MO 63130

$U \cdot S \cdot A$.

KLEIN RICHARD I DR

KITT PEAK NAT OBS

$P$ BOX 26732

TUCSON AZ 85726

$U . S . A$.

KLEMPERER W K DR

DIV ASTRONDMICAL SC

NAT SCIENCE FOUND

1800 G STREET N W

U. S.A.

KLINGLESMITH DANIEL A DR GSFC 671

GREENBELT MD 20771

U.S.A.

KNACK RDGER F DR

DEPT EARTH SPACE SCS

SUNY

STONY BROOK NY 11794

U.S.A.

KNEER $F$ DR

KIEPENHEUER INST

FUER SONNENPHYSIK

SCHOENECK STR 6

D-7800 FRE IBURG-BR

GERMANY, F.R

KNUDE JENS KIRKESKOV DR COEENHAGEN UNIV OBSERVATORY

OSTER VOLDGADE 3

DK - 1350 COPENHAGEN-K

DENMARK

\author{
KIRBIYIK HALIL DR \\ MIDDLE EAST TECHNI - \\ CAL UNIVERSITY \\ ANKARA
}

KISELEV ALEXEJ A DR

PULKOVO OBSERVATORY

196140 LENINGRAD

U.S.S.R.

KISSELL KENNETH E DR

TORY RW

W-P AFB OH 45433

U. S.A.

KITCHIN CHRISTOPHER R DR

HATF IELD PQLYTECHNIC

OBSERVATORY

BAYF OROBURY

HERTFORD HERTS

U.K.

KJELDSETH MOE DLAF DR

INSTI TUTE OF THEORE-

TICAL ASTROPHYSICS

UNIVERSITY OF OSLO

BLINDERN OSLO 3

NORWAY

KLECZEK JOS I P DR

ASTRONOMICAL

INST I TUTE

25165 ONDRE JOY

KLE I NMANN DOUGLAS E DR

CDNGARDEN ST

CAMBRIDGE MA 02138

U.S.A.

KLEPCZYNSKI WILLIAM J DR

US NAVAL OBSERVATORY

WASHINGTON DC 20390

U.S.A.

KLIORE ARVYDAS JOSEPH DR JPL

8OO OAK GROVE DRIVE

PASADENA CA 91103

U.S.A.

KNAFLICH H B MS

BOEING CQMPANY

AERDSPACE GROUP

$M$ S 88-21 POB 3999

SEATTLE WA 98124

U. S.A.

KNIFFEN DONALD A DR

NASA-GSFC

GREENBELT MD 20771

U.S.A.

KO HSIEN C PROF

DEPI OF ELECTRI I AL

OHIO ST UNIVERSITY

COLUMBUS OH 43210

U.S.A. 


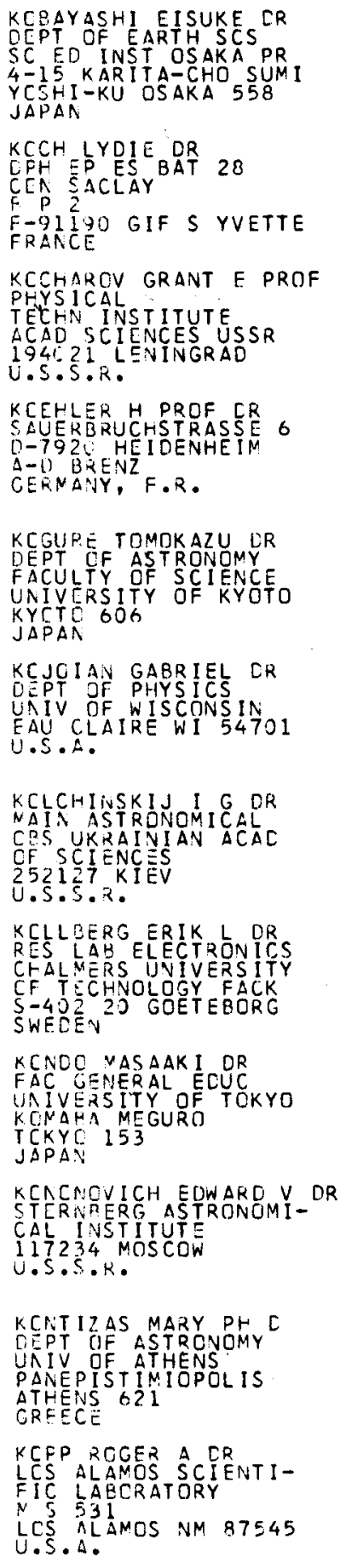

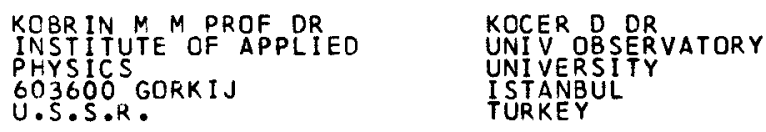

KOCER D OR

UNIV OBSERVATORY

UNI VERSITY

ISTANBUL

TURKEY

KODA IRA KEIICHI A PRDF DEPT OF ASTRONOMY FACULTY OOF SCIENCE UNIVERSITY OF TOKYO JAPAN

KOEHLER JAMES A PROF PHYSICS DEPT UN IV OF SA SKATOON SASKATOON S7N OWO CANADA

KOHL JOHN L DR

CENTER FOR

ASTROPHYSICS

6) GARDEN ST

CAMBR IDGE MA 02138

U.S.A.

KOKUR IN YUR IJ L DR

LEBEDEV PHYS INST

LESR ACAD OF SCS 53

117924 MOSCDW

U.S.S.R.

KOLESNIK L N DR MAIN ASTRONOMICAL OES UKRAINIAN ACAD OF SCIENCES $252127 \mathrm{KIEV}$

U.S.S.R.

KOMAROV N S DR ODESSA STATE UNIVERSITY ASTRONIMICAL OQSERVATCRY 270014 DDESSA

U.S.S.R.

KONDO YOJI DR

CODE 685

GODDARD SPACE FL CTR GREENBELT MD 20771 $U . S . A$.

KONOPLEVA V P DR MAIN ASTRONDMICAL OBS UKRAINIAN ACAD OF SCIENCES U.S.S.R.

KOPAL ZDENEK PROF DEPT OF ASTRONOMY UNIVERSITY OF MAVCHESTER MANCHESTER M13 9PL U.K.

KOPYLOV I M OR SPECIAL ASTROPHYSI CAL OBSER VATORY USSR ACADEMY OF SCI $357140 \mathrm{~N}$ ARKHYZ U.S.S.R.
KOCHAR R K DR

INDIA INSTITUTE OF

ASTROPHYSICS
BANGALORE $560 \quad 034$ I NDI A

KOECKELENBERGH ANDRE DR OBSERVATOIRE ROYAL DE BELGIQUE

AVE CIRCULAIRE B-1 1 gO BRUXELLES BELGIUM

KOESTER DETLEV DR INST THEOR PHY STERN NEUE UNIV PHYS ZENTR OLSHAUSENST GEBN $61 \mathrm{C}$ D-2300 KIEL I GERMANY, F.R.

KOHOUTEK LUBOS DR HAMBURGER STERNWARTE GOJENSBERG SWEG $0-2050$ HAMBURG 80 GERMANY, F.R.

KOLACZEK BARBARA DR PLAN. GEODESY DEPT UL BARTYCKA 18 OO-716 WARSAW POLAND

KOLESOV A K DR

LENINGRAD STATE UNIVERSITY AS TRO

199178 LENINGRAD

U.S.S.R.

KOME SARDFF MAX M D SC CSIRO DIVISION OF RADI OPHYSICS

BOX 76

EPPING NSW 2121

AUSTRALIA

KONIN $\vee V D R$

NIKDLAEV BRANCH OF

THE MAIN ASTRONOMI -

CAL OBSERVATORY 327000 NIKOLAEV U.S.S.R.

KONTI ZAS EVANGELOS.PH D NAT OBS OF ATHENS

ASTRONOMICAL INST

THISSION

A THENS 306

GREECE

KOPECKY MI LOSLAV DR

ASTRONOMICAL INST CZECHOSLOVAK ACADEMY OF SCIENCE 25165 ONDREJOV DBS CZECHOSLOVAKIA

KDRCHAK A A DR INSTITUTE OF TERRESTRIAL MÁGNETISM AND I CNOSPHERE

142092 ACADEMGORODOK U.S.S.R. 


\section{KCRLYLEWSKI KAZIMIERZ DR. SKLCCOWSKIES 6 PCLANO}

KCKCVYAKOVSKIJ YURIJ P DR SPECIAL ASTROPHYS OR

AI I HXIJ ARKHYZ

$35714 J$ STAVROPCLSKIJ

K.RAJ

U.S.S.R.

KCSTYAKOVA ELENA B CR

STLRNEERG STATEN

127234 MCSCOW

U.S.S.R.

\section{KCTHARI D.S DR . \\ UAIVERSITY OF CELHI \\ NEW $\Gamma E L H I$}

INDI $A$

KCURGANCFF VLADIMIR PROF $2 G$ AVE PAUL APPELL

F 75014 PARIS

FRANCE

KCVACS AGNES DR

PELI I PHYS I

$H=4913$ DEBRECEN

HUNGARY

\section{KCVAR N SEDR \\ PHYSICS DEPT \\ HCUSTCN TX 77004 \\ U.S.A.}

KENAL CHARLES THOMAS DR DEPT UF ASTROPHYSICS

CALTECH

U.S.A.

KCZHEVNIKOV N I OR

TREACMICAL INSTI TUTE

117234 MOSCOW

U.S.S.R.

KCZLGWSKI MACIEJ DR

ASTRCNOMICAL

CESERVATORY

AL UJAZDOWSKIE 4

UU 478 WARSZ AWA

CLAND

KRAFT ROEERT P PROF

LICK DASERVATURY

UNIVERSITY

CF CALIFORNIA

SANTA CRUZ CA 95064

U.S.A.

KRANJC A OR

OSS ASTRONOMICO

I-40100 BCLOGNA

IT A $A$ Y Y
KORMENDY JOHN DR

DOMINION ASTROPHYS-

ICAL OBSER VATORY

5071 W SAANICH ROAD

VICTOR IA BC V $8 \times 3 \times 3$

CANADA

KOSIN GENNAD I J S DR

PULKOVO OA SERVATORY

196140 LENINGRAD

U.S.S.R.

KOSTYLEV K V DR

ENGELHARDT ASTRONOMICAL OB SERVATORY 422526 KAZAN

U.S.S.R.

KOTOV V A DR

CRIMEAN A STROPHYSI-

CAL OBSER VATORY USSR

ACAD OF SCI NAUCHNIY

334413 CR IMEA

U.S.S.R.

KOUTCHMY SERGE DR

INSTITUT D ASTROPHY-

SIQUE CNRS

98 BIS BD ARAGO

F-750I 4 PARIS

FRANCE

KOVAL I K DR

MAIN ASTRONOMICAL

OBSERVATORY

GOLOSEEVO

$252127 \mathrm{KIEV}$

U.S.S.R.

KOVAR ROBERT $P$ DR

9666 E ORCHARD DR

U.S.A.

KOYAMA SHIN PROF DR

KAGAWA UN I VERSITY

SA IWA I CHO

TAKAMATSU 760

JAPAN

KOZIEL KAROL PROF DR UL 22 LIPCA 16

POLAND

KOZYREV NIKOLAJ A DR PULKOVO OESERVATORY 196140 LEN INGRAD

U.S.S.R.

KRAICHEVA Z DR

DEPT OF ASTRONOMY

BULGAR IAN AC OF SCS

7TH NOVEMBER

BULGARIA

KRASINSKY GEORGE A DR

INST THEORET ASTRON

USSR ACAD OF SCS

10 KUTUZOV QUAY

192187 LENINGRAD

U.S.S.R.
KOROLKOV DMITRY $V$ PROF DR

PULKOVO OBSERVAT ORY

196140 LENINGRAD

U. S.S.R.

KOSTINA LIDIJA D DR

PULKOVO OBSERVATORY

196140 LENINGRAD

U.S.S.R.

KOTELNIKOV V A ACAD

INSTITUTE OF RAOIO

AND ELECTRONICS

USSR ACADEMY OF SCI

U.S.S.R.

KOTSAKIS DEMETRIUS PRDF HIPPOCRATES STR IB9 A THENS-708

GREECE

KOVACHEV B J DR

ACAOEMIE BULGARE

SECTION ASTRONOMIE

RUE 7 NOVEMBER NO 1

BULG IARIA

KOVALEVSKY JEAN DR

CERGA

ÁV COPERNIC

F-06130 GRÁSSE

FRANCE

KOVETZ ATTAY PROF

DEPT OF PHYSICS AND

ÁSTRONOMY

TEL AVIV UNIVERSITY

I SRRAEL

KOZAI YOSHIHIDE PROF

TOKYO ASTRONOMICAL

OBSERVATORY

OSAWA MI TAKA

TOKYO

JAPAN

KOZLOVSKY B Z DR

ASTRONOMY

TEL-AVIV UNI VERSITY

RAMAT AVIV

I SRAEL

KRAEMER GERHARD DR

ERLENWEG 24

D-74OO TUEB INGEN

GERMANY, F.R.

KRAMER KH N DR

ODESSA STATE UNIVER-

SI TY ASTRONOMICAL

OBSERVATOR

270014 ODESSA

U.S.S.R.

KRAT VLADIMIR A PROF DR PULKOVO OBSERVATORY 196140 LENINGRAD

U.S.S.R. 
KRAUS JCHN D PRCF RAOIC CBSERVATORY 2015 INEIL AVE

CELUMUUS OH 43210

U.S.A.

KREINER JERZY NAREK OR

UL SPOLDZIELCOW $8 / 52$

$30-6 Z 2$ KRAKOW 47

PCLANC

KRESAK LUBOR DR

ASTRC IUNICAL INST

SAV

DURRAVSKA CESTA II/A

$893 \%$ ERAT ISLAVA

CLFCHIDSLOVAK IA

KKINOV EUGEN L PROF METEURITE COMM ITTEE

ACAE ENY OF SCIENCES

i 7313 MOSCOW

KRISTENSEIVLEIF KAHL DR

UAIV OF AARHUS

NY NLNKGADE

CK-QJJJ AARHUS C

CENMARK

KRISTIANSSCN KRISTER

GE PRCF
SEPT CF PHYS ICS
SCELVEGATAN I 4

SCELVEGATAN 14
$S-22362$ LUND

KRCGLAHL W S CRR ISTRC NCMY

UNIVERSITY KENTUCKY

LEXINGTCN KY 40506

U.S.A.

KRCAECRG PHILIPP DR

UA IVERSIIT

SCARBCRCUGH CCLLEGE

TCRCATC MIC IAS

KRUEOER E PROF

$\checkmark I A$ MAURD MACCHI 65

I $-201 \mathrm{~J}$ M MLANC

ITALY

KSANTOMALITIL V CQ

SFAC RESEARCH INSTI

SCI ACES

I17 1 J MCSCOW

U.S.S.R.

KUSCTA JUN DR

KASAY OESERVATORY

YANASHINA

KYCTO 607

JAPAK

KUHI LECNARD V PROF

ASTRE VONY DEPT

UAIVERSITY OF CLLIF

EERKELEY

U.S.A.
KRAUSE F DR

ZNTRINST ASTROPHYSIK

ASTROPHYS OBS

TELEGRAFENBERG

DOR - 15 POTSEAM

$G \cdot D \cdot R$.

KREISEL E DR

STERNW BABEL SBERG

ROSA LUXEMBURGST $17 A$

DDR- 15 I 2 POT SDAM

$G \cdot D \cdot R$.

KRESAKOVA MARGITA OR

ASTRONOMICAL INST

SAV

DUBRAVSKA CESTA $11 / A$

8930 BRATISLAV

CZECHOSLOVAKIA

KR I SHNA SWAMY K S DR

ASTROPHY ICS GROUP

TATA INSTITUTE

COLABA

BOMBAY 400005

INDIA

KR ISTENSON HENRIK DR

SLAANBAER SVAEGEN 9

$S-381$ DO K.ALMAR

SWEDEN

KR IVSKY L DR

$\triangle S T R O N O M I C A L$

INSTITUTE

25165 ONDREJOV

CZECHOSLOVAKIA

KRON GERALO E OR

4IG N BERTRAND ST

U.S.A.

KROOK M UR

HARVARD COLLEGE OBS

CAMBR IDGE MA O2138

U.S.A.

KRUSZEWSKI ANDRZEJ PROF

A STRONOM ICAL

OBSERVATORY

AL UJAZDOWSKIE 4

$0 O-478$
POLAND

KUBIAK MARCIN A DR

WAR SAW UN IVER SITY

OBSERVATORY

AL UJAZOOWSK IE 4

PO- 478

KUORITZKI ROLF-PETER PH D

INST F THEOR PHYSIK

OLSHAUSENSTRASSE

DE 2300 KIEL.

KUIJPERS JAN DR

STERREKUNDIG INST

UTRECHT

THE NETHERLANDS
KRAUSHAAR WILLIAM L PROF DEPT OF PHYSICS

1150 UNIVERSITY AVE

MADI SON WI 53706 AVE

U.S.A.

KREMPEC JANINA DR

N. COPERNICUS AST CTR

ASTROPHYSICAL LAB.

CHOP INA $12 / 18$

87-100 TORUN

POLAND

KRIEGER ALLEN S DR

AMERICAN SCIENCE AND

NG I NEER ING

955 MASSACHUSETTS AV

CAMBRIDGE MA O2139

U.S.A.

KRISHNAN THIRUVENKATA DR $132-A$ LLOYDS ROAD

GOPALAPURAM

MADRAS 600086

I ND I A

KRISTIAN JEROME DR

HALE OBSERVATORIES

813 SANTA BARBARA ST

PASADENA CA 91101

U.S.A.

KRI 2 SVATOPLUK DR

DEPT. OF ASTRONOMY

CHARLES UNIVERSITY

SVEDSKA 8

150 OnO PRAGUE

CZECHOSLOVAKIA

KRON KATHERINE GORDON

PINECREST QBS

416 N BERTRAND ST

FLAGSTAFF AZ 86001

U.S.A.

KRUEGER ALBRECHT DR

ZENTRALINSTI TUT FUER

SOLAR-TERR.PHYSIK

DDR-II99 BERLIN

G.D.R.

KRZEMINSKI W OR

COPERNICUS ASTRONO-

MICAL CENTER

UL BAR TYCKA 18

OD-716 WARSAW

POLAND

KUBICELA ALEKSANDAR DR

ASTRONOMSKA OPSERV

VOLGINA

11050 BEOGRAD

YUGOSLAVIA

KUEHNE CHRISTOPH F DR

CARL ZEISS COMPANY

POSTFACH 35/36

D=7082 OBERKOCHEN

GERMANY, F.R.

KUIPER THOMAS H H DR

JET PROPULSION LABC-

RATORY 183 B-365

PASADENA CA 91103

U. S. A. 


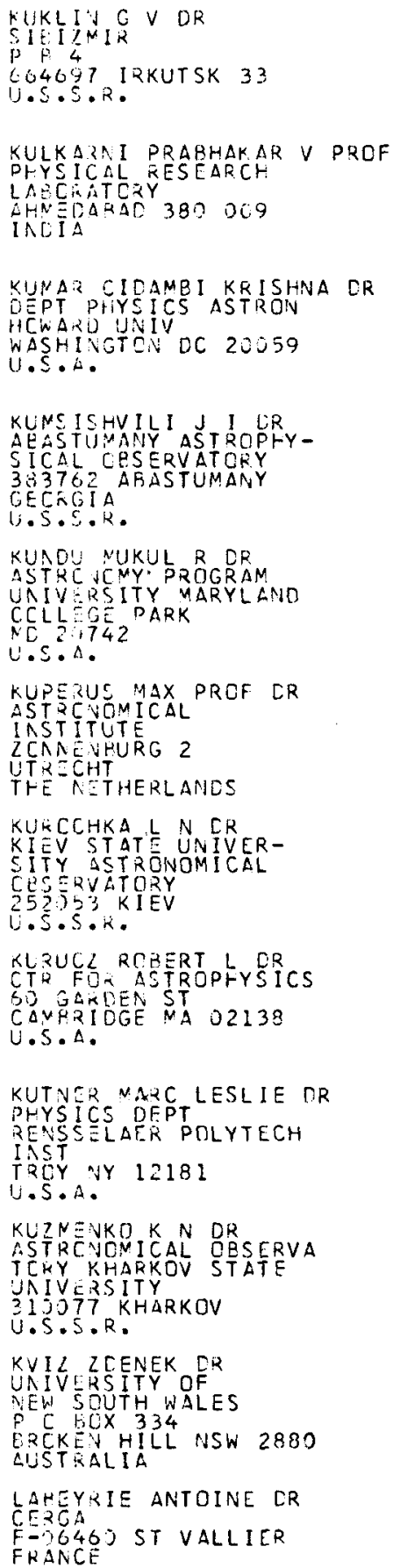

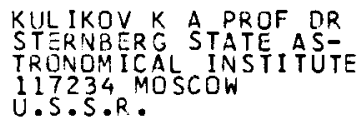

KULSRUD R M DR

ASTROPHYSICAL SCS

PR INCETON UN IVERSITY PRINCETON NJ 08540 U.S.A.

KUMAR S S PROF

DEPT OF ASTRONUMY

UNIV OF VA POB 3818

CHARLOTTESVILLE

$\checkmark A 22903$

U.S.A.

KUINCHEV PETER OR DEPT OF ASTRONOMY FACUL TY OF PHYSICS

1126 SOF IA

BUL GAR IA

KUNKEL WILLIAM E DR OBS NACIONAL

RUA GENERAL BRUCE

586 2C O8

RIO DE JANEIRO GB BR AZ IL

KUPPERIAN JAMES E DR CENTER

CODE 410

GREENBELT MO) 20771

U.S.A.

KURT V G DR

SPACE RESEARCH INSTI

TUTE USSR ACADEMY OF

SCIENCES

117810 MOSCOW

$U . S . S . R$.

KUSHWAHA R S PROF

DEPT OF MATHEMATICS

UNIV OF JODHPUR

JODHPUR RAJ

INDIA

KUTTER G SIEGFRIED DR THE EVIRGREEN STATE

OLYMPIA WA 98505

U.S.A.

KUZMIN ARKADII D

PROF DR SC

ACADEMY SC

U.S.S.R.

KWEE K K DR

STERREWACHT

POSTBUS 9513

THE NETHERLANDS

LABEYRIE JACQUES DR CENTRE DES FAIBLES RADIOACTIVITE

LAB M IXTE CNRS-CEA 91190 GIF SUR YVETTE
KULIKOVSKIJ J G DR STERNBERG STATE AS117234 MOSCOW

U.S.S.R.

KUMAJGOROOSKAYA RAISA DR

SPECIAL ASTROPHYS OE

NI ZHNIJ ARKHYZ 357140 STAVROPOLSKIJ KRAJ

U.S.S.R.

KUMSIASHVILY MZIA I DR

ABASTUMANI ASTRO-

PHYSICAL OBSERVATORY

383762 GEORGIA

U.S.S.R.

KUNOT WOLFGANG PROF DR

INST F ASTROPHYSIKU

AUF DEM HUEGEL 71

$D=5300$ BONN 1

GERMANY, F.R.

KUNZEL HORST TUT FUER

SCLARTERRESTRISCHE

PHYSIK

DDR- 15 POTSDAM

$G \cdot D \cdot R$.

KURIL-CHIK V N DR

STERNBERG STATE AS-

TRONOMICAL INSTITUTE

117234 MOSCOW

U. S. S.R.

KURTH R DR

PROF OF MATHEMATICS

GEORGIA INST OF

TECHNOLOGY

ATLANTA GA 30332

U. S.A.

KUSTAANHEIMO PAUL E PROF

DIA E 451

DANMARKS TEKNISKE

HOJSKOLE

OK-2805 LYNGBY

DENMARK

KUTUZOV S A OR

LENINGRAD STATE UNI-

OESERVATORY

199178 LENINGRAD

U.S.S.R.

KUZMIN GRIGORI G PROF

I APHA ESTONIAN

ACADEMY OF SCIENCES

TORAVERE

202444 TARTU

U.S.S.R.

KWOK SUN DR

HERZBERG INST ASTRO

NAT RES CQUNCIL CAN

IOS SUSSEX DRIVE

OTTAWA ONT KIA ORG

LABORDE GEORGES DR

OBSERVATOIRE

DE MEUDON

F-92I90 MEUDON

FRANCE 
LARRUM NORMAN R MR CSIRC DIVISION OF KAUIDPHYS

EFPING NSW 2121

AUSTRALIA

LACRCUTE PIERRE A PROF F-2IOJO DIJUN

FRANCE

LAFCN JEAN-PIERRE J DR CBSERVATOIRE DE MEUDCN F-9219U MEUDON FRANCE

LAHULLA J FORNICS DR CBSERVATORIO

ALFONSCXII 5

MADRID 7

SFAIN

LANG FREDERICK K PROF PAYSICS DEPT

UNIVERSITY OF

ILLINCI

UREANA

LANBERT DAVID L PROF

CEPT OF ASTRONOMY

UNIVERSITY OF TEXAS

R L MOCRE HALL,

U.S.A.

LANLA ERICH E CR

ASTRCNOMISCHES INST

STERNW UNIV BONN

AUF DEN HUE

GERMLIVY, F.R.

LANDE KENNETH PROF

PHYS ICS DEPT

UNIVERSITY OF

PHILADELPHIA PA19104

U.S.A.

LANCI CEGL INNOCENT I

EGIDIU"OR

CSSERVATORIO ARCETRI

LARGC FERMI

I -50125 FIRENZE

ITALY

LANDNAN DONALC ALAN DR

INST FCR ASTRONOMY

HALEAKALA OBS

$P$ COX 157

KULA MAUI HI 96790

U.S.A.

LANG JAMES DR

ASTRCPHYS ICS RES DIV

CULHAM L

CXCN OXI4 30B

U.K.

LANTCS PIERRE

FES 150 MEUDON

FRANCE
LABS DIETRICH PROF

LANDESSTERNWARTE

KOENIGSTUHL

D-6900 HEIDELEERG 1

LAOA CHARLES JOSEPH DR

STEWARD OBS

UNIV OF ARIIONA

TUCSON AZ 85721

U.S.A.

LAGEROVIST ALBIN PROF

INSTITUTE OF PHYSICS

VANAD I SVAEGEN

$S-11346$ STOCKHOLM

SWEDEN

LALA PETR DR

ASTRONOMICAL INST

25165 ONDRE JOV

CZECHOSLOVAK IA

LAMB SUSAN ANN DR

DEPT OF PHYSICS

UNIV OF MISSOUR

8001 NATURAL BRG RD

ST LOUIS MO 6312

U.S.A.

LAMBRECHT HERMANN PROF DR SCHILLERGAESSCHEN 2

DDR-69 JENA

$G . D \cdot R$.

LAMY $P$ OR

LAB D'ASTRON SPAT

TRAVERSE DU SIPHON

LES-TROIS-LUCS

F- 13012 MAR SEILLE

LANDECKER PETER BRUCE DR

AEROSPACE CORP

SPACE SCIENCES LAB

$P$ D BOX 92957

LOS ANGELES CA 90009

U.S.A.

LANDI - DESSY J UR

OBSERVATOR IO NACIO-

NAL ARGENTINO

CORDOBA

ARGENTINA

LANDOLT ARLD U PROF DEPT PHYS AND ASTRON LOU ISIANA STATE

UN IVERSITY

BATON ROUGE LA 70803

U.S.A.

LANG KENNETH R ASST PROF

DEPT OF PHYSICS

TUFTS UN IVER SI TY

U.S.A.

LAPAZ LINCQLN DR

IINST OF METEORITICS

UN IV OF NEW MEXICD

CAMPUS PO $80 \times 23$

ALRUOUERQUE NM 87131

U.S.A.
LACLARE F MR

A VENUE COPERNIC

F-06130 GRASSE

FRANCE

LAFFINEUR MARIUS MR

FI BLVD BRUNE

FRANCE

LAHIRI N C

INDI AN ASTTRON EPH UN

IND I AN MET DEPT

NEW ALIPORE CALCUTTA INDIA

LAMB DONALD QUINCY JR DR CTR FQR SPACE RES

BLDG $37-555$

MIT

CAMBRIDGE MA 02139

U.S.A.

LAMBECK KURT PROF

AUSTRALIA NAT UNIVER

SITY RE SEARCH SCHOOL

EARTH SC PO BOX 4

CANBERRA 2600

AUSTRALIA

LAMERS H J G L M DR

ONDERZOEK

BENELUXLAAN 21

UTRECHT

THE NETHERLANDS

LANCASTER BROWN PETER

41 INDEN CLOSE

ALDEBURGH SUFFOLK

U. K.

LANDECKER THOMAS L OR DOMINION RADIO ASTRO PHYSICAL OBSERVATORY $B O X 248$

PENTICTON BC VZA $6 K 3$ CANADA

LANDINI MASSIMO PROF OSSERVATORIO ARCETRI

LARGO FERMI

ITALY 25 FIRENZE

LANDSTREET JOHN D PROF DEPT OF ASTRONOMY

UNI VERS I TY OF

WESTERN ONTARID

LONDON ONT NGA $5 B 9$ CANADA

LANGER WILLIAM DAVID DR DEPT PHYSICS ASTRON $G R C-B$

UNIV OF MASS

AMHERST MA D 1003

U.S.A.

YAPOINTE S M DR UNI VERSITE DE QUEBEC 2875 BOUL LAURIER CANADA 
LAPUSHKA K K DR

LATVIAN STATE UN IVER

SITY ASTRONOMICAL

CESERVATORY

226098 RIGA

U.S.S.R.

\section{$\angle A R I C D R$ \\ LAE NATIONALE CI \\ RALI GASTRDNOMI \\ I A IRNER IO 46 \\ IT ALY}

LARSCN RICHARD $B$ PROF

YALE UNIVERS ITY

CESERVATORY BOX 2023

YALF STATION

NEW HIAVEN CT O652?

U.S.A.

LASKARIDFS PAUL C ASSPROF

UNIVIRSITY OF ATHENS

PANEPISTIMIOPOL IS

ATHENS 621

LATHAY DAVID W QR

CTH F ASTROPHYSICS

CO GAROEN ST

CANIRICGE MA 02138

U.S.A.

LATYPOV A A DR

ASTRCNCMICAL INST

UZREKI AN ACAO DF SC

700035 TASHKENT

U.S.S.R.

LAURENT RERTIL E PROF

INST ITUT E FOR

VANACISVAEGEN 9

$S-11346$ STOCKHOLM

SWEDEN

LAUSTSEN SVEND OR

DK $-9, O, 1$ ARHUS IT

DEAMARK

LAVAL ANNIE DR

CPSERVATOIRE DE

NARS ILLE

2 PLACE LE VERRIER

FRANCE

LAVRUKHINA A K PROF DR

IASTITUTE OF GEQCHE-

CHEMISTRY

I17334 MOSCOW

U.S.S.R.

LAZCVIC JOVAN P PROF

DEPT OF ASTRONOMY

FACULTY OF SCIENCES

STUOENTSKI TRG 16

11000 EEOGRAO

YUGCSLAVIA

LE SQUEREN ANNE-MARIE DR CESERVATOIRE

CE NEUDON

FRANCE
LAQUES PIERRE OR

OPSERVATOIRE

DU P IC DU MIDI

F-65200 BAGNERES

DE B I GORRE

FRANCE

LAR INK JOHANNES PROF DR HAMBURGER STERNWARTE

BERGEDORF

GOJENBERGSWEG 78

D-2050 HAMBURG 80

GERMANY, F.R.

LARSSON LEANDER GUNNAR

PRDF

LUND OBSERVATORY

SVANEGATAN 9

$S-22224$ LUND

SWEDEN

LASKER BARRY M DR

CERRO TOLOLO

INTERAMER ICAN OBS

CASILLA 630

LA SERENA

CHILE

LATOUR JEAN J

JILA

UNIV OF COLORADO

BOULDER CO 80309

FR ANCE

LAUBERTS ANDR IS DR

AS TRONOM ICAL

OBSERVATORY

BOX 515

S- 75 I 20 UPPSALA

LAURENT C DR

$\overline{\mathrm{B}} \stackrel{\mathrm{P}}{\mathrm{P}} \mathrm{N}^{\mathrm{P}} 10$

F-9 1370 VERRIERES

LE-BUISSON

LAUTERBORN D PROF DR

HAMBUR GER STERNWARTE

$D-2050$ HAMBURG 80

GERMANY, F.R.

LAVDOVSK I J VICTOR V DR

PULKDVO OBSERVATORY

196140 LENINGRAD

U.S.S.R.

LAWRENCE G M DR

SASP $P$ SC IENCE BLDG

UNIV OF COLORADO

BOULDER CO 80302

U.S.A.

LE CONTEL JEAN M

OBSERVATOIRE DE NICE

LE MONT GROS

B $P 252$

FRANCE

LEA SUSAN MAUREEN DR

ASTRONOMY DEPT

UN IV OF CAL I FORN I A

BERKELEY CA 94708

U.S.A.
LARGE MICHAEL I DR

SCHOOL OF PHYSICS

SYDNEY NSW 2006

AUSTRALIA

LARSON HAROLD P DR

LUNAR AND PLANETARY

LABORATORY

UNI YERSITY ARI ZONA

TUC SON AZ 85721

U.S.A.

LASHER GORDON JEWETT DR

IBM WATSON RES CTR

U.S.A.

LASOTA JAN PIOTR DR

COPERNICUS ASTRONO-

MICAL CENTER

UL BARTYCKA 18

OO 716 WARSAW

POLAND

LATTIMER JAMES M DR

DEPT OF ASTRONOMY

UNIV DF ILLINOIS

URBANA IL 61801

U.S.A.

LAUNAY J M DR

OBS DE MEUDON F-92190 MEUDON

LAUSBERG ANDRE DR B-462 I RETINNE

LAUTMAN D A DR SMITHSONIAN ASTROPHYSICAL

60 GARDEN STREET

CAMBRIDGE MA OLI 38 U.S.A.

LAVROV M I DR ENGELHAROT AASTRONOMICAL OBSERVATORY 422526 KAZAN U.S.S.R.

LAYZER DAVID PROF

HARVARD COLLEGE

OBSERVATORY

60 GARDEN ST

CAMBRIDGE MA 02138

U.S.A.

LE POQLE RUOOLF $S$ DR

STERREWACHT

POSTBUS 9513

2300 RA LEIDEN

LEBEOINETS $V N$ OR ASTRONOMICAL COUNCIL PYATNITSKAYA UL 48 109017 MOSCOW

U.S.S.R. 
LEELANC YOLANDE OR DE NLUOCN F-92190 MEUDON FRANCE

LECKRONE DAVID S CR GCLOARL SPACE CCCE 671 GREENBELT MD 20771 U.S.A.

LEE $P$ D DR

LCUISI INA STATE UNIV

BATCR ROUGE LA 70803

U.S.A.

LEER EGIL PROF UURCRAL OBSERVATORY $P$ C ACX 953 $N=9$ JUI TROMSO NCRWAY

LECG THOMAS H CR

CF ASTROPHYSICS

NAT RES COUNC CANADA

CTTANA CNT KIA ORG

CANADA

LE I HCWITZ ELIAM CR

ANO ASTRONOMY

TEL AYIV UNIVERSITY ISTA AVIV

LEINERT CHHISTOPH DR KCENISSTUHL

C-693 HEICELEERG 1

GEKNANY, F.R.

LENAITRE GERARD R DR CUSERVATOIRE

2 PLACE LEVERRIER

F-13jI4 MARSEILLE

FRAACE

LEORAT JACQUES CR

CESERVATOIRE

LE NEUDCN

FRANCE

LERCY JEAN LOUIS

LESEVATCIRE

F-65 DO BAGNERES

FE BICRRE

LELAG CHUN MING DR

DEPT OF PHYS ICS

REASSELAER POLYTECHN

INSTITUTE

TRCY NY 12181

U.S.A.

LEVASSEUR-REGOURC

ANAY-CHANTAL DR

SERV AERD CNRS PP 2

F-91375 VERRIERES

LE EUISSON

FRANCE
LEBOVITZ NORMAN R PROF

MATHEMAT ICS DEPT

UNIV OF CHICAGO

5734 S UNIV AVE

U.S.A.

LEDERLE TRUDPERT DR

ASTRONOMISCHES

RECHEN INSTI TUT

MUENCHHOF STR I 214

GERMANY, F.R.

LEE SEE-WOO DR

DEPT EARTH SC IENCES

KYUNGPOOK NATIONAL

UN IVER SI TY

TAEGU CITY

REPUBLIC OF KOREA

LEFEBVRE MICHEL DR

CNES-GRGS

18 AVE E BELIN

F-31055 TOULOUSE CED

FRANCE

LEHNERT $80 \%$ PROF

DEPT PLASMA PHYSICS

TECHNOLOGY

S- 10044 STOCKHOLM 70

SWEOEN

LEIGHTON R B PROF

HALE OBSERVATORIES

CAL IF INST OF TECHN

PASADENA CA 91125

U.S.A.

LEL IEVRE S OR

F-92190 MEUDON

FRANCE

LEMKE DIETRICH DR MP I FUER ASTRONOMIE KOENIGSTUHL

D- 6900 HE IDELBERG 1

GERMANY, F.R.

LEPINE JACQUES R D DR

CRAAM $/ O N / C N P Q$

RUA CEARA 290

SAO PAULO

BRAZ IL

LESTER JOHN B DR ER INDALE COLLEGE UNIV OF TORONTO MISSISSAUGA LSL IC6 CANADA

LEUNG KAM CHING PRDF BEHLEN OBSERVATORY

UN IV OF NEBRASKA

LINCOLN NB 68588

U.S.A.

LEVATO ORLANDO H DR

CALLE 38 NO 463

1900 LA PLATA

ARGENTINA
LECAR MYRON OR

CTR FOR ASTROPHYSICS

GO GARDEN ST

CAMBRIDGE MA 02138

U.S.A.

LEDQUX P J PROF

INST D ASTROPHYSIQUE UNI VERSITE DE LIEGE B-420O COINTE DUGREE BE LGIUM

LEE TERENCE J DR

U.K. I NFRARED

TELLESCOPE UNIT 900 LEILANI STREET

U.

LEFEVRE JEAN DR OBSERVATOIRE DE NICE B P 252

FRANCE

LEIBACHER JOHN DR

SPACE ASTRONONOMY GR

LOCKHEED 52-12/202

3251 HANOVER ST

PALO ALTO CA 94304

U.S.A.

LEIKIN G A OR

ASTRONOMICAL COUNCIL

USSR ACAD OF SCI

PYATNI TSKAYA UL 48

109017 MOSCOW

U.S.S.R.

LEMAIRE PHILIPPE DR

$L P S P$ CNR

B $P, 10$

FE 1370 VERRIERES

FRANCE

LENA PIERRE J PROF

OB SERVATOIRE

DE MEUDON

F-92190 MEUDON

FRANCE

LEQUEUX JAMES DR

OB SERVATOIRE DE

PAR I S MEUDON
$F-92190$ MEUDON

FRANCE

LETFUS VOJTECH DR

ASTRONOMICAL INST

CZECH ACAD SCIENCES

25165 ONDREJDV

OB SERVATORY

CZECHOSLOVAKIA

LEVALLOIS J J DR

INSTITUT GEOGRAPHI -

QUE NAT I ONAL

2 AVE PASTEUR

F-94160 SAINT-MANDE

FRANCE

LEVIN B J DR

A STRONOMICAL COUNCIL

PYATNITSKAYA UI SC

109017 MOSCOW

U.S.S.R. 
LEVISE RANDQLPH H DR CO CARCEN ST

CDNHRICGE MA 92138

U.S.A.

LENIN WALTER H G PROF

MIT 37-627

CANKRICGE MA 22139

U.S.A.

\section{CALIFORNIA UNIV \\ US31 NITHWORTH RQ \\ U.S.A.}

LIESKE JAY H CR

CALIF INST TECHN

JET PRQPULS ION $L A B$

4 हO GAK GROVE DRIVE

PASACEN

U.S.A.

LILLEY EDWARC A PROF

HARVART COLLEGE

CESERVATORY

GI GARLEN ST 02138

U.S.A.

LIN COUGLAS E CR

MAC INGLEY ROAD

CANBRICGE CB3 JHA

U.K.

LINDHLAD PER OLCF PROF

STCCKHCLM
CESERVATORY

$S-133, i$

SALTS JOERADEN

SWECEN

LINGENFELTER RICHARC E

PREF OF ASTRONOMY

UAIV CF CALIFORNIA

LCS ANGELES CA 90024

U.S.A.

LANELL ALBERT P PRUF

DEPT OF ASTRONOMY

ANE ASTROPHYSICS

MICHIJAN STATE UNIV

EAAS ING MI 48824

U.S.A.

LIPCVETSKY $V A$

SPECIAL ASTROPHYS OB

ZELEVCUK

35714 J STAVROPOLSKIJ

KRAJ

U.S.S.R.

LITTLE C GORDON

CHIEF DIV 87 T ANCARCS

RN 3OI RADID BLDG

BCLLCER CO 80302

$U \cdot S . A$.

LIU SOU-YANG OR

CENPUTER SCIENCES CO

SYSTEM SCIENCES DIV

A728 COLESVILLE ROAD

5 ILVER SPG MD 20910

U.S.A.
LEVY EUGENE H DR

LUNAR ANDETARY SCI

UN IVER SITY ARIZONA

TUCSON AZ 85721

U.S.A.

LEWIS BR IAN MURRAY DR CARTER OQSER VATORY

PO BOX 2909

WELL INGTON

NEW ZEALAND

LIDDELL U MR

NASA LUNAR AND PLAN-

ETARY PROGRAM OFF OF

SPACE SC AND APPL

WASHINGTON DC 20546

U.S.A.

LILLER MARTHA H DR

HARVARO COLLEGE

OBSERVATORY

CAMBR IDGE MA 02138

U.S.A.

$\angle I L L I E C F D R$

(DSTRON

DOULOR COLORADO

U.S.A.

LINCOLN J VIRGINIA MISS

WDC-A FOR SOLAR-

TERRESTRIAL PHYSICS

NOAA D 63

BOULDER CO 80303

U.S.A.

LINFOOT E H DR

THE OBSER VATOR IES

MADINGLEY ROAD

CAMBRIDGE CB 3 OHA

$U \cdot K$.

LINK FRANCOIS PROF

INSTITUT

D ASTROPHYSIQUE

98 BIS BLVO ARAGO

F-75014 PAR IS

FRANCE

LINNIK $V P$ PROF DR

MAIN A STRONOMICAL

OBSERVATORY PULKOVD

196140 LENINGRAD

$U . S \cdot S \cdot R$.

LIPP INCOTT SARAH LEE DR

SWAR THMORE COLLEGE

SWAR THMORE COLLEGE

U.S.A.

LITTLE LESLIE T DR

ELECTRON ICS LAB

CANTERBURY

KENT CT2 TNT

U.K.

LIVINGSTON WILLIAM C

UBSERVATURY

$P$ O BOX 26732

TUCSON AZ 85726

U.S.A.
LEVY JAQUES R DR

DE PARIS GI AV L OBSERVATOIRE F-75014 PARIS FRANCE

LI JUNG Y

ACLADEMY OF SCIENCES

PYONGYANG 'S REP OF KOREA

LIEBSCHER D E DR

ZNTRINST ASTROPHYSIK

STERNW BABELSBERG

ROSA LUXEMBURGST 17 A

DDR- 1502 POTSDAM

$G \cdot D \cdot R$.

LILLER WILLIAM PROF

CTR F ASTROPHYSICS

60 GARDEN ST

CAMBRIDGE MA 02138

U.S.A.

LIN CHIA C PROF MIT OF MATHEMATICS

77 MASSACHUSETTS AVE CAMBRIDGE MA O2139 U.S.A.

LINDBLAD BERTIL A DR

SVANEGATAN 9

SWEDEN 24 LUND

LING CHIH-BING DR

ACADEMIA SINICA

P O BOX NO 143

TAIPE I

LINKE RICHARD ALAN DR

BELL LABS

CRAWFORD HILL LAB

HOLMDEL NJ 07733

U.S.A.

LINSKY JEFFREY L DR

JILA

UNIVERSITY OF

COLORADO

BOULDER CO 80309

U.S.A.

LITTLE ALEC G ASSOC PROF

PHYSICS DEPT

UNIVERSITY OF SYDNEY

SYDNEY.NSW 2006

LITVAK M DR

SMITHSONIAN ASTRO-

PHYSICAL OBS

CAMBRIDGE MA 02138

U.S.A.

LIVSHITS M A DR

INSTI TUTE OF TERRE-

STRI AL MAGNETISM AND

I ONOSPHERE

142092 AKADEMGORODOK

U.S.S.R. 
LLCYOIEVANS THOMAS DR $S$ A ASTRONOMICAL OBS CESERVATORY

CAPE 7935

LCCHTE-HOLTGREVEN W PROF

INST I TUT FUER EXPPERI

NENT ALPHYSIK CER

UA IVERS IT AET

GERNALY KIEL

LCDEN KERSTIN R LR

STCCKHCLM

CESERVATORY

$S-133$ O O

SALTSJCERADEN

$S W E D E N$

LCUGAIR M S DR

ASTRCV ROYAL FOR

SCETLAND

ECINSURGH EH9 3JH

U.K.

LCPEZ JOSE A ING

FELIX AGUILAR

AV BENAVIDEZ 8175 OE

5477 MARGUE
ARGE INTA

LCRTET MAFIE CLAIRE

LPSERVATUIRE

DEE NEUDCN

$F-92190$ MEUDON

FRANCE

LCUGHHEAD RALPH E DR

NAT ICNAL NEASUREMENT

LAEOKATCRY

FRADFI LO RD

LINCF I

AUST:ALIA

LCVAS FRGNCIS JCHN DR

vlecular spectr

CIJ 545

NAT UUREAU STANCAROS

WASHIVCTOV DC 20234

L.S.A.

LEVELL SIR BERNARD PROF

CFESIITE SKII $9 P L$

U.K.

LCYCLA PATRICID DR

UNIVESIDAD DE CHILE

CACI LA $36-D$

CFIL

LU PHILLIP K CR

LEPT PHYSICS ASTRON

ISI WHITE ST

CANEUKY CT J6R10

U.S.A.

LUECHIVF FISICA

G GALILEI

$1-35130$ PADOVA

ITALY
LD KWOK-YUNG DR

OWENS VLY RADIO OBS

CAL TECH 102-24

PASADENA CA 91125

U.S.A.

LOCKE JACK L DR

HERZBERG INSTI TUTE

NF ASTROPHYSICS

NAT RES CQUNC CANADA

CANADA

LODEN LARS OLOF PROF

ASTRONOMICAL OBSERVA

TORY BOX 515

S-751 20 UPPSALA

LONGMORE ANDREW J

63 MOAT VIEW

ROSLIN MIDLOTHIAN

U.K.

LOPEZ-ARROYO M

DBSERVATOR IO

ASTRONOMICO

ALFONSO $\times 115$

MADR ID

SPAIN

LOSCO LUCETTE DR BESANCON F-25030 BESANCON CECEX

FRANCE

LOUISE RAYMQND DR

OESERVATOIRE

DE MARSEILLE

F- 13004 MARSEILLE

FRANCE

LOVAS MIKLOS

KONKOLY OBSERVATORY

$B \cap \times 67$

HUUNGARY 25 BUDEST

LOW FRANK J DR

4949 CALLE BARRIL

TUCSON

LOZINSKAYA T A DR TRONOMICAL INSTITUTE 117234 MOSCOW

U.S.S.R.

LUE JAN DR

C O E SO SHE IMERSTR 17
SCHLEISSHEIMERST

GERMANY, F.R.

LUCKE PETER B DR

CH-1290 SAUVERNY GE

SWITZERLAND
LOCANTHI DOROTHY DAVIS DR $218 O P I N E C R E S T$ DR.

U.S.A.

LOCKWOOD G WESLEY DR

LOWE BOX 1260

FLAGSTAFF AZ 86002

U.S.A.

\section{LOHMANN WERNER PROF \\ ASTRONOMI SCHES \\ RECHEN INS I I TUT \\ MOENCHHOF STR 1214 \\ GERMANY HEI DEL LE}

LOPEZ GARCIA ZUELMA L DR

ORS ASTRONOMICO

FELIX AGUILAR

AV BENAVIDEZ 8175 OE

5407 MARQUESADO S J

ARGENTINA

LOREN ROBERT BRUCE DR

MILLIMETER WAVE OBS

MCDONALD DBS

UNIV OF TEXAS

FT DAVIS TX 79734

U.S.A.

LOTOYA N A DR

PHYSICAL INSTITUTE

USSR ACA

SCIENCES

117924 MOSCOW

U.S.S.R.

LOULERGUE $M$ DR

FBS 2190 MEUDON

FRANCE

LOVELACE RICHAPD V E DR

SPACE SCIENCES

ITHACA NY I 4853

U.S.A.

LOWE ROBERT P DR

DEPT OF PHYSICS

UNI $V$ WESTERN ONTARID

LONDON ONT NGA $3 K 7$

CANADA

LOZINSKI J A M DR

ASTRONOMICAL COUNCIL

USSR ACADEMY OF SCI

PYATNI T SKAYA

U.S.S.R.

LUCAS R DR

ECOLE NORMALE SUP

24 RUE LHOMONO

FRANCE

LUCY LEON B PROF

COLUMBI A UNIVERS ITY

NEW YORK NY IOOZ27

U.S.A. 


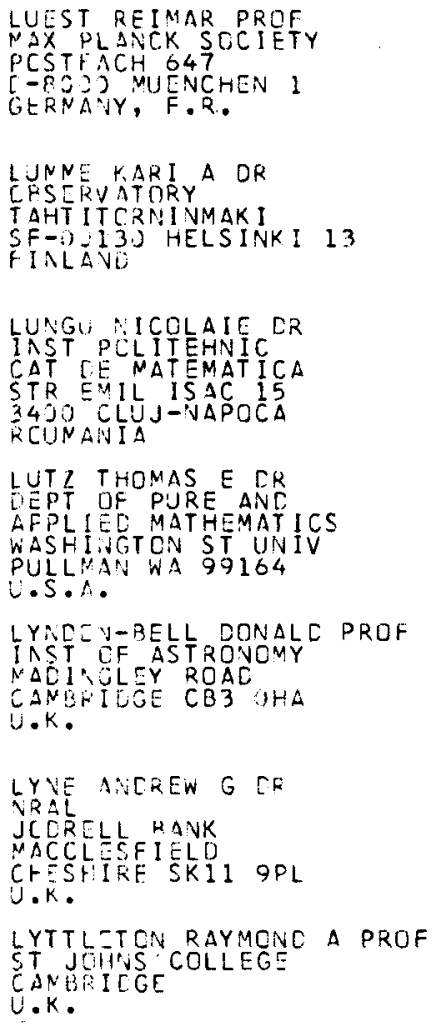

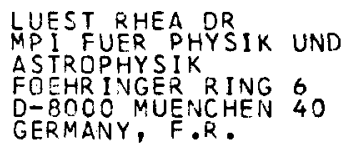

LUNDQUIST CHARLES A DR

SPACE SCTENCES

LABORATO

CODETSSSLIE AL 35812

U.S.A.

LUTZ BARRY L DR

LOWELL OBSER VA TORY

P O BOX 1269

FLAGSTAFF AZ 86002

U.S.A.

LUUD LAUR I DR

TARTU ORSERVATORY 202444 ESTONIA

U.S.S.R.

LYNDS BEVERLY T DR

KITT PEAK NATIONAL

OESER VATORY

$P$ O BOX 26732

TUCSON AZ 85726

U.S.A.

LYNGA COSTA DR

LUND OBSERVATORY

SVANEGATAN 9
S- 22224 LUND

SWEDEN

LYUTY VICTGR M DR STERNEERG INSTITLTE NAUCHNYJ

334413 CRIMEA

U.S.S.R.

MACCONNELL DARRELL J DR CENTRO DE

APARTADO 264

MER IDA

VENEZUELA

MACHADO JOSE MA B DR TECHNICAL UNIVERSITY OF LISEON

AV DA IGREJA 1710 LISBOA 5

PORTUGAL

MACLEOD JOHM M DR

HERZBERG INSTI TUTE

OF ASTROPHYSIC S

NAT RES COUNC CANADA

OTTAWA DNT KIA ORG

CANADA

MACR IS CDNSTANTIN I PRDF RSAAM ACADEMY ATHENS ANAGNOSTOPOULOU 14 ATHENS 136

GREECE

MADORE BARRY FRANCIS DR DAVID DUNLAP DBS

UN IV OF TORONTO

RICHMOND HL L 4C 4Y6

CANADA
LUKACEVIC ILIJA -

FACULTY OF SCIEA

DEPT OF MECHANICS

IIOOO BEOGRAD

YUGOSLAVIA

LUNEL MADELEINE DR

DBSERVATOIRE

DE -69230 SAINT GENIS

FAVAL

LUT 2 JULIE H DR

DEPT UTIEATHEMATICS

WASHINGTON STATE

UNIVERSITY

PULLMAN WA 99164

U.S.A.

LUYTEN WILLEM J PROF

SPACE SCIENCE CENTER

MINNESOTA

MINNEAPOLIS MN 55455

U.S.A.

LYNDS ROGER C OR

CB SERVATORY

TUCSON AZ 26732

U.S.A.

LTTKENS EJNAR OR

SKOLGATAN 33 R

$S=75221$ UPPSALA

MACCACARO TOMMASO DR

LAB RADIDASTRONOMIA

VIA IRNERID 46

I 40126 BOLOGNA

MACQONALO GEOFFREY H DR

ELECTRONICS LAB

UNIVERSITY OF KENT

KENT CT2 TNT

U.K.

MACHALSKI JERZY DR

30 -019 KRAKOW

POLAND

MACQUEEN ROBERT M DR

HIGH ALTI TUDE

PO BOX 300

BOULDER CO 80307

U.S.A.

MACY WILLIAM WRAY DR

INST FOR ASTRONCMY

UNIV OF HAWAII

2879 WOODLAWN DRIVE

HONOLULU HI 96822

U.S.A.

MAEDER ANDRE DR

OBSERVATOI RE

DE GENEVE

CH-1290 SAUVERNY GE

SWI TZERLAND 
MAEHARA HIDEO DR TCKYO ASTRONOMICAL OOSERVATORY

CSAWA MITAKA

TEKYO 181

JAPAN

NAGALASHVILI N L DR

$\triangle E A S T U M A N I$ ASTROPHY-

SICAL OBSERVATORY

383762 ABASTUMANI

GEERGIA

NAGNARADZE N G DR

UNIVERSITY

380043 TBILISI

$U . S . S \cdot R$.

MAHMCUL FAROUK M A A DR HELWAN OBSERVATORY HELWAY CAIRO

$\triangle R A B$ REP. OF EGYPT

NAILLARD JEAN-PIERRE CR

CESERVATOIRE

F-92190 MEUDON

FRANCE

NAKARQVA ELENA A DR

CAL INSTITUTE

117234 MOSCOW

U.S.S.R.

MALAISE DANIEL J CR

IASTITUT

E ASTRCPHYSIQUE

$B-42$ COINT E-OUGREE

BELGIUM

NALIN DAVID F DR

$\triangle A C L C-A U S T R A L I A N$ OBS

EFPING NSW 2121

AUSTRALIA

NALLIA EOWARD A OR

CEPT OF ASTROPHYSICS

SCUTH PARKS ROAD

U.K.

NALTEY PER PROF

INST THEOR PHYSICS

UNIVERSITY OF OSLO

$P C R^{2} O X \quad 1029$

ELINOEKN OSLO 3

NCRWAY

NANMANC A DR

I 36 . 12 AS I AGO VIC

IT TLLY

NANCUSCI-SANT I CR

CAPODIMONTE ASTRONO-

NICAL CBSERVATORY

$\checkmark$ IA NOIARIELLO 16

I-EUI3I NAPOL

ITALY
MAETZLER CHR ISTIAN OR

INST APPL IED PHYSICS

SIDLERSTRASSE 5

CH- 3012 BERN

MAGALHAES ANTONIO A S DR

OBS ASTRON DE BARROS

UNIV DO PORTO

MONTE DA VIRGEM

4400 VILA NOVA GAIA

PDR TUGAL

MAGUN ANDREAS DR

INST APPLIED PHYSICS

SIDLER STRASSE 5

CH- 3012 BERN

MAHRA H S DR

UTTAR PRADESH ST OBS

MANORA PEAK

NAINITAL 263129

INDIA

MAITZEN HANS M DR

UN IVER SI TAETS-

STERNWARTE

TUERK ENSCHANZ STR 17

$A-1180$ WIEN

AUSTRIA

MAK ITA MITSUGU DR

TOKYO ASTRONOM ICAL

OBSERVATORY

OSAWA MITAKA

TOKYO 181

JAPAN

MALAKPUR IRADJ DR

INST OF GEOPHYSICS

TEHRAN UN IVER SITY

AMIRABAD SHOMALI

TEHRAN

IRAN

MALINOVSKY MONIQUE DR

B P NO 10

F-91370 VERR IERES LE

BUISSON

MALLIK D C VDR

INDIAN INSTITUTE OF

ASTROPHYSICS

BANGALDRE $560 \quad 034$

INDIA

MALVILLE J MCKIM PROF

DEPT ASTROGEOPHYSICS

UN IVERS ITY OF

COLORADO

BOULDER CO 80302

U.S.A.

MANARA ALESSANDRO A DR

OSSERVATOR IO ASTRONO

MICO DI MILANO

$\checkmark$ IA BRERA $2 B$

I 20121 MILAND

MANDEL STAM $S L$ PROF

INST OF SPECTROSCOPY

USSR ACACEMY DF SC

142092 AKADEMIGORD

DOK MOSCOW REGION

$U \cdot S \cdot S \cdot R$.
MAFFEI PAOLO PROF

OSSER VATORIO

ASTROF I SICO

I $=95125$ CATANIA

I TALY

MAGNAN CHRISTIAN DR

LAT BIS BLVD ARAGO

F-75014 PARIS
FRANCE

MAHDY HAMED A DR

HELWAN OBSERVATORY

HELWAN-CAIRO
ARAB REP. OF EGYPT

MAIHARA TOSHINORI DR

DEPT OF PHYSICS.

KYOTO UNI VERSITY

JAPAN

MAKARENKO EKATERINA N DR

ASTRONOMICAL OBS

PARK SHEVCHENKO

270014 ODESSA

U.S.S.R.

MALACARA DANIEL

INAOE

AP POSTAL 216 Y5I

TONANTZINTLA

PUEBLA PUE

MEXICO

MALAROQA STELLA M DR

QBS ASTRONOMICO

PASEO DEL BOSOUE

1900 LA PLATA

ARGENTINA

MALI TSON HARR IET H MS

NASA GODDARD SPACE

FLIGHT CENTER

CODE 601

GREENBELT MO 20771

U.S.A.

MALMQUIST K GUNNAR PROF

ST QLOF SGATAN IO A

SWEDEN 21 UPPSAL

MAMEDREJLI G D DR SHEMAKHA ASTRDPHYS I -

CAL OBSERVATORY

373243 AZERBAIDZAN

U.S.S.R.

MANCHESTER RICHARD N DR CSIRO RADI OPHYS ICS

P $O$ BDX 76

EPPING NSW 2121

AUSTRALIA

MANFROI D JEAN DR

INST D'ASTROPHYS I QUE

UNIVERSITE DE LIEGE

AVENUE DE COINTE 5

$B-4200$ COINTE-OUGREE 


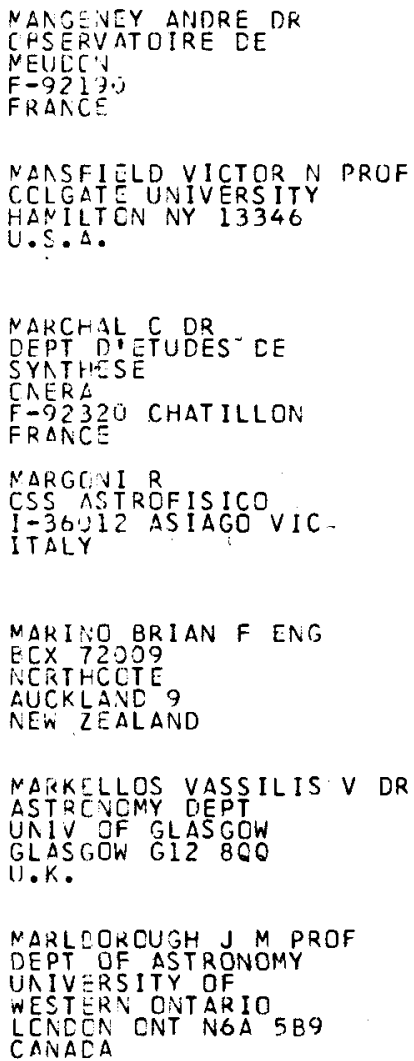

MANNINO GIUSEPPE PROF IST I TUTO MA TEMATICO $\checkmark I A$ CAMPI 181 I- 41100 MODENA

MARAN STEPHEN P DR NASA-GSFC CODE 680 GREENBELT MD 20771 U.S.A.

\section{MARCUS ELLA PROF \\ OBSER VATO IRE ASTRONOM IQUE ARGINT BUCAREST 75212 ROUMAN IA}

MARIK MIKLOS DR. ASTRONOM ICAL DEPT. KUN BELA TER 2 H- 1083 BUDAPEST HUNGARY

MARK JAMES WAI-KEE DR RES LAB ELECTRONICS MASS INST TECHNOLOGY

BLDG 26 RM 215

CAMER IDGE MA O2139 U.S.A.

MARKKANEN TAPIO

OESERVATORY

TAHT I TORN INMAK I SF-OO 130 HELSINKI 13 FINLAND

MAROCHNIK L S PROF DR USSR ACAD OF SCS SPACE RESEARCH INST PROF SO JOSNA A U.S.S.R.

MAKQUES MANUEL $N$ DR OB SER VA TOR IO A STRONOMICO TAPADA DA AJUDA LISBOA 3 PORTUGAL

MARSDEN PHILIP L PROF DEPT OF PHYSICS UN IVERS ITY DF LEEDS LEEDS LS2 9JT

U.K.

MARTEL MAR IE-THERESE DR OBSERVATOIRE DE 69230 SAINT GENIS LAVAL FRANCE

MARTIN NICOLE DR OBSERVATOIRE DE MAR SE I LLE 2 PLACE LE VERR IER F-13004 MARSEILLE FRANCE

MARTIN WILLIAM C DR AI67 P.HYSICS NAT IONAL BUREAU OF STANDARDS WASHINGTON DC 20234 U.S.A.
MANRI QUE WALTER T PROF OBS ASTRONOMICO FELIX AGUILAR $\triangle V$ BENAVIDEZ 8175 OE 5407 MARQUESADÓ S J ARGENTINA

MARASCHI LAURA DR VI A CELORIA 16 ITALY

MARGON BRUCE H PROF

ASTRONOMY DEPT

UNI VERSITY OF CALIF
LDS ANGELES CA 90024 U.S.A.

MARILLI ETTORE DR

OSS ÁSTROOFISICO CITTA UNIVERSITARIA I-95I25 CATANIA

MARKARIAN B E DR BYURAKAN ASTROPHYSICAL OBSERVATORY 378433 ARMENIA U.S.S.R.

MARKOWITZ WILLIAM DR 2800 E SUNRISE BLVD U.S.A.

MAROV MIKHAIL YA DR INST OF APPLIED MATH USSR ACAD OF SCS MIUSSKAYA SQ 4 MOSC OW A-45 U.S.S.R.

MARRACO HUGO G DR OBS ASTRONOMICO PASEO DEL BDSQUE 1900 LA PLATA ARGENTINA

MARSH JULI AN C D HATF IELD POLYTECHNIC OE SERVATORY BAYFORDRURY NR HERTFORD HERTS SGI3 $8 L D$ U.K.

MARTIN DEREK H PROF QUEEN MARY COLLEGE MILE END ROAD LONDON E1 4 NS U. $K$.

MARTIN PETER G PROF DEPT OF ASTRONOMY UNI VERSI TY TORONTO TORONTO ONT MSS IAT CANADA

MARTIN WILLIAM L DR ROYAL GREENWICH OBS HERSTMONCEUX CASTLE HAILSHAM

E SUSSEX BN2T IRP

U. K. 
NARTIN-LORON M OR 14 NACRID I

SPAI

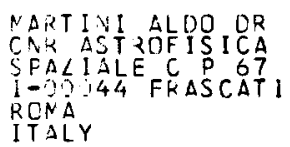

VARTYYCV D YA PRCF DR STERNAERG STATE ASTRCNOMICAL INS

U.S.S.R.

\section{MAKX S DR \\ ZRTRINST ASTROPHYSIK KARL SCHWARLSCHILD \\ CESEKVATORIUM \\ CDR-64. I TUT ENBURG G. C.R. \\ NESNCU FRANCOISE OR 28 ALLEE GAMBAUCERIE FOI 19}

MASSEVICH A G DR

ASTRE NOMICAL COUNCIL

PYATVITSKAYA UL 48

1.17 MOSCOW

U.S.S.R.

ATHEWS WILLIAM C PROF

LICK CESER VATORY

SAIVERSITY OF CALIF

U.S.A.

MATIAGIN VALERY S DR

ASTREPFYSICAL INST

480068 ALMA-ATA

U.S.S.R.

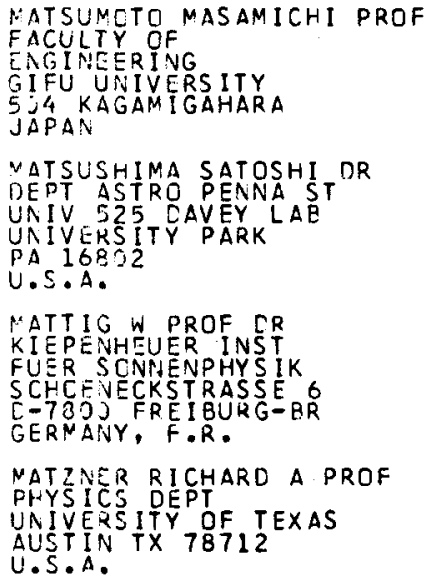

MARTINET LOUIS PROF ORSERVATOIRE

DE GENEV

C.H- 1290 SAUVERNY GE SWITZERLAND

MARTINS DONALD HENRY DR

DEPT PHYSICS ASTRON

UN IV OF GEORGIA

30602

U.S.A.

MARVIN UR SULA B DR

CENTER FOR

ASTROPHYSICS

60 GARDEN S

CAMBR IDGE MA 02138

U.S.A.

MASAN I A PROF

OSS ASTRDNOMICD DI

BRERA

VIA BRERA 28

I-201OU MILANO

MASNOU J L DR

SECT DIASTROPHYSIQUE

CEV SACLAY

F- 91120 GIF-S-YVETTE

FRANCE

MASURSKY HAROLD PROF

US GEDLOGICAL SUR VEY

BR OF ASTRO STUDIES

2255 NORTH GEMINI DR

FLAGSTAFF AZ $86 \mathrm{CO}$

U.S.A.

MATHEWSON DONALD S DR

MT STROMLO

WOOEN POACT 2606

AUSTRALIA

MATSON DENNIS L DR

JPL $183-501$ OOE DRIVE

PASADENA CA 91203

U.S.A.

MATSUMOTO TOSHIO DR

DEPT OF PHYSICS

NAGOYA UNIVERS ITY

FUROCHO CHIKUSAKU

NAGOYA 464

JAPAN

MATTEI JANET AKYUZ DR

AAVSO

187 CONCORD AVE

CAMBRIDGE MA 02138

U.S.A.

MATTILA KALEVI DR

OBSER VATORY

TAHT I TORN INMAKI

SF-00130 HELSINKI 13

FINLAND

MAUCHERAT-JUUBERT MME

LAB DIASTRON SPAT

TRAVER SE OU SIPHON

LES TROIS LUCS

F- 13012 MARSE ILLE

FRANCE
MARTINEZ M DR

INST DE ASTRONOMIA

UNAM

APT POSTAL 70-264

MEXICO DF

MEXICO

MARTRES MARIE-JOSEPHE

OB SERVATOIRE DE

PARI S-MEUDON

F-92190 MEUDON

FRANCE

MARX GYORGY PROF.

EOTVOS UNIVERSITY

PUSKIN U 5

H-1088 BUDDAPEST

HUNGARY

MASLOWSKI JOZEF DR

ASTRDNOMICAL

CBSERVATORY

UL ORLA I 71

30 - 244 KRAKOW

POLAND

MASON HELEN E DR

DEPT APPLIED MATH

AND THEORET PHYSICS

SILVER STREET

CAMBRIDGE

U.K.

MATAS VLADIMIR R DR

ASTRONOMISCHES

RECHEN INSTI TUT

MOENCHHOF S TR $12-14$

GERMANY, F, R.

MATHIS JOHN $S$ PROF

DEPT OF ASTRONOMY

UNI VERSITY OF

WI SCONSIN

MADISON WI 53706

U.S.A.

MATSUDA TAKUYA PROF

DEPT AERONAUTIC ENG

KYOTO UNI VERSITY

YOSHIDAHONMACHI

SAKYOKU KYOTO 606

JAPAN

MATSUOKA MASARU DR

INST SPACE AERON SC

UNI VERSITY OF TOKYO

KOMABA MEGURO

TOKYO 153

JAPAN

MATTHEWS THOMAS A DR

ASTRONOMY PROGRAM

UNI VERSI TY MARYLAND

COLLEGE PK MD 20742

U.S.A.

MATVEYENKO L I DR

SPACE RESEARCH

USSR ACADEMY OF SCI

117810 MOSCOW

U.S.S.R.

MAUDER HORST PROF DR

A STRONOMI SCHES INST

WALDHAUSER STR 64

D-T400 TUEBINGEN

GERMANY, F.R. 


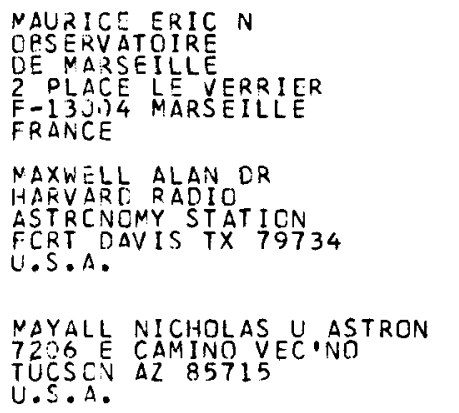

NAYER URSULA DR ASTRCNUN ISCHES INST CER UNIVERS I TAET D-740\% TUEBINGEN GERNAINY, F.R.

NALUREK THACDEUS JOHN DR DEPT UF ASTRONOMY

UNIV OF TEXAS

AUSTIN TX 78712

NCAOAN W BRUCE CR

UAIVERS ITY OF SYCNEY

SYCNEY NSW 2006

AUSTRALIA

NCCABE NARIE K MS

INST FOR ASTRONOMY

2600 WCCDLAWN CR

HENCLULU HI 96822

U.S.A.

MCCARTHY MARTIN F DR

SPECCLA VAT ICANA

CITT $A$ DEL VAT ICANC

NCCLUQE ROBERT C PROF

DĆNINICN ASTROPHYSIC

CESERVATORY

507 I SAANICH ROAO

VICTCRIA BC V $8 X \quad 3 \times 3$

CANACIA

MCCRACKEN KENNETH G DR

CSIRE

$P C$ BUX 136

AC RTH RYDE NSW 2113

AUSTKALIA

MCCKLSKY RICHARD. E DR

CENTER FOR

ASTREPHYSICS

CANPIICEE MA 02138

U.S.A.

NCECNALD FRANK B CR

CCCE 500

GREE VBELT MD 22771

U.S.A.
MAVRIDIS L N PROF

DEPT GED ASTRONOMY

UNIVERSITY

DF THESSALONIKI

THESSALLNIK

GREECE

MAY J ING

OBS RADIOASTRONOMICO

UNIV DE CHILE

CASILLA 68

MAIPU

MAYER CORNELL H

SPACE SCIENCE DIV

NAVAL RESEARCH LAB

CODE 7130

WASHINGTON DC 20375

U.S.A.

MAYFIELD EARLE $B$ OR

5536 M ICHELLE DR
TORRANCE CA 90503

U.S.A.

MALZITELLI ITALO DR

LAR ASTROFIZICA SPAZ

P O B 67

I =000 44 FRASCATI

MCALISTER HAROLD A DR

DEPT OF PHYSICS

GEORGIA STATE UNIV

UN IVER S I TY PLAZA

ATLANTA

MCCARROLL RONALD PROF

LAB D ASTROPHYSIQUE

UN I VER S I TE

DE BORDEAUX I

$F-33405$ TALENCE

FRAVCE

MCCLAIN EDWARD F

4133 MAPLE RD

MORNINGSIDE MD 20023

U.S.A.

MCCLUSKEY GEORGE E JR DR

DIV OF ASTRONOMY

DEPT OF MATHEMATICS

LEHIGH UN I VER SITY

U.S.A.

MCCRAY RICHARD DR

JILA

UNIVERSITY OF

COLTRADO

BOULDER CO 80309

U.S.A.

MCCULLOCH P M DR

DEPT OF PHYSICS

UNIV OF TASMANIA

HOEART TASNAANIA

AUSTRAL IA

MCDONALD J K PETRIE DR

OOMINION ASTROPHYSI-

CAL ORSERVATORY

5071 SAANICH ROAD

VICTOR IA BC V $8 \times 3 \times 3$

CANADA
MAX CLAIRE ER DR

I VERMORE LABORATORY

D O BOX 808

LI VERMORE CA 94550

U. S.A.

MAYALL MARGARET W

5 SPARKS ST

CAMBRIDGE MA 02138

U.S.A.

MAYER PAVEL DR

CHARLES UNIVERSITY

SVED SKA 8

15000 PRAHA 5

MAYOR MICHEL DR

OBSERVATOIRE

DE GENE V

CH- 1290 SAUVERNY GE

SWI TZERLAND

MAZZUCCONI FABRIZ OODR

OSSERVATOR IO ARCEYRI

LARGO FERMI 5

I -50125 FIRENZE

MCBREEN BRIAN PHILIP DR

PHYSICS DEPT

UNIV COLLEGE

BELFIELD

IRELAND

MCCARTHY DENNIS D DR US NAVAL OBSERVATORY WASHINGTON DC 20390

U.S.A.

MCCLINTOCK JEFFREY E DR

TUTE OF TECHNOLOGY

BLDG 37-52

CAMBRIDGE MA 02139

U.S.A.

MCCORD T B DR

INST F ASTRONOMY

2680 WODDLAWN DR

HONOLULU HI 96822

U.S.A.

MCCREA WILLIAM H PROF

ASTRONOMY CENTRE

SUSSEX UNI VERSITY

BRIGHTON BNI 9 QH

U.K.

MCCUTCHEON WILLIAM H PROF

DEPT OF PHYSICS

UNI VERS ITY OF Q C C

VANCOUVER BC VST IWS

CANAOA

MCDONNELL J A M DR

SPACE SCIENCE LAB

UNIVERSITY OF KENT

CANTERBURY

KENT CT2 7NT

U.K. 
NCEC NDUGH THOMAS R DR
1239 CERDOVA RM 206 PAST CCRDQVA RM 206 j. . . 1 .

NCGEE RICHARDX CR CSIKC DIVISION OF RADIOPHYSICS

PC BCX 70

EFPIVQ VS' 2121

AUSTRALIA

MCKE CHF ISTOPHER F PROF

PHYS ICS DEPT

JNIVERSITY OF CALIF

EERKELEY

MCKENYA LAWLOR SUSAN

N P CIR

ST PATRICKS COLLEGE

$I$ KEL $A J C$

VCLEAN CONALDJ JR

CS IRE

F Pid 70

CFPIVG NSW 2121

YCAALLY EEREK CR

UNIVLRSITY OF LONCON

CBSERVATORYY

MILL HILL PARK

LC $C$ CI NW $720 \mathrm{~S}$

$\mathrm{U} \cdot \mathrm{K}$.

NCWHIRTER R W PETER DR

ASTREPHYSICS RES. CIV

CULFAM LAB

$\triangle B$ IABOCN

CXCN OXI4 $30 B$

$\mathrm{U} \cdot \mathrm{K}$.

NEACOWS A JACK PROF

AST RCNCMY AND HISTO-

RY CF SCIENCE DEPT

UAIVERSITY LE ICESTER

U.K.

MEFFROY JEAN F RROF DR

INST MATHEMAT IQUES

UNIV OES SCIENCES ET

TECHIV CU LANGUECOC

FRANCE

MEHLTHETTER JPOCR

FUER SONNENPHYSIK

SCFOENECKSTR 6

GERNANY, F.R.

NEINIG MANFREC DR

ZENTEALINSTITUT

TELEGRAFE.VE, ERG 17 A

C. $R=I 5$ POTSDAM

$G . C . R$.

MELBCURNE WILLIAM G DR 48 JO [OK GROVE CRIVE PASAUENA CA 91103

U.S.A.
MCELROY M B DR

KITT PEAK NAT OBS

$950 N$ CHERRY AVE

TUCSON $A Z 85726$

U.S.A.

MCGIMSEY BEN Q JR DR

DEPT CIF PHYSICS

GEORGIA STATE UNIV

UNIVERSITY PLALA

ATLANTA GA 30303

U.S.A.

MCKEITH CONAL D DR

17 ADELA IDE PARK

BELFAST BT9 GFX

U.K.

MCK INLEY DONALD W R DR 1889 FAIRMEADOWW CR DTTAWA ONT

CANADA

MCLEAN IAN $S$ DR

LUNAR AND PLANET LAB

UNIV OF ARIZONA

TUCSON $A Z 85721$

U.S.A.

MCNAMARA DELBERT H DR

DEPT OF PHYSICS AND

ASTRONOMY

BR IGHAM YOUNG LNIV

PROVO UT 84602

U.S.A.

MEABURN J DR

THE UN IVER SI TY

MANCHESTER MI3 9PL

U.K.

MFBOLO U OR

MPI FUER RADID-

AUF DEM HEUGEL 69

DE-53OS EONN I

MEGESSIER CLAUDE DR

OBSERVATOIRE PAR IS

GI AVE OBSERVATOIRE

F-75014 PARIS

FRANCE

MEIN PIERRE

DE MEUDON

5 PLACE JANSSEN

F-92190 MEUDON

FRANCE

MEISEL DAVID D DR

DEPT OF PHYSICS AND

ASTRONOMY

STATE UNIV COLLEGE

GENESEO NY 14454

U.S.A.

MELCHIOR PAUL J PROF

QBSERVATOIRE ROYAL

DE BELGIQUE

AVENUE CIRCULAIRE 3

B-I180 BRUXELLES

BELGIUM
MCGEE JAMES D PROF

IMPEICS DEPT

LONDON SW7

U.K.

MCINTOSH BRUCE A DR

OF ASTROPHYSICS

NAT RES COUNCIL CAN

OTTAWA ONT KIA ORG

CANADA

MCKEITH NIALL ENDA DR ST PATRICK'S COLLEGE MAYNOOTH CO KILDARE IRELAND

MCLAREN ROBERT A DR

DEPY OF ASTRONOMY

UNIV OF TORONTO

TORONTO ONT MSS IAT

CANADA

MCMULLAN DENNIS DR

ROYAL GREENWICH

OQSERVATORY

HAI LSHAM

EAST SUSSEX BN2T IRP

U.K.

MCVITTIE GEORGE C PROF

74 OLD DOVER

KENT CTI 3 AY

U.K.

MEAD JAYLEE MONTAGUE DR

NASA-GSFC

GODEENBELT MD 20771

U. S.A.

MEEKS M LITTLETON DR

LINCOLN LABORATORY

P O BOX 73

LEXINGTON MA 02173

U.S.A.

MEGRELISHVILI T G PROF

ABASTUMANI ASTROPHY-

SICAL OBSERVATORY

GEORGIA

U.S.S.R.

MEINEL ADEN B PROF

OPTICAL SCIENCES CTR

UNIVERSITY OF

AR I ZONA

UU. S.A.

MEKLER YURI DR

DEPT OF GEOPHYSICS

TEL-AVIV

I SRAEL

MELNIKOV OLEG A PRQF DR

PULKOVO OBSERVATORY

196140 LENINGRAD

U.S.S.R. 


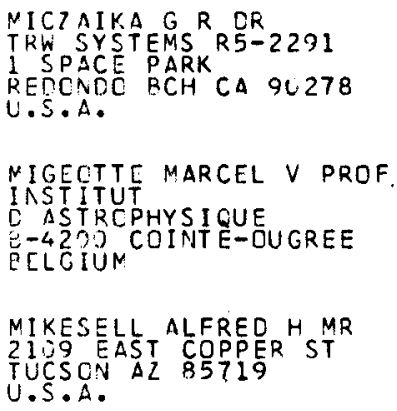

MILES HOW ARD G MR CLVEVTRY CV 3 5PE

MILLS BERNARD Y PROF SCHCCL CF PHYSICS UAIVERSITY OF SYDNEY SYCNEY NSW 2006 AUSTRALIA

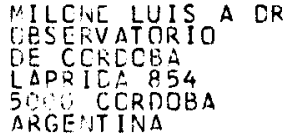

NIAN YOUNG KEY CR KCREAN NAT I O'JAL CPSERV DTQRY

KANGNAN-KU

SECUL $134-03$

REPU ILL OF KOREA
MIDDLEHURST BARBARA M MS - LUNAR AND PLANETARY

INSTI TUTE

3303 NASA ROAD 1

U.S.A.

MIHAILA IERONIM M DR

BUCHAREST UNIVERSITY

ACADEMIE I 14

70109 BUCHAREST

RGUMAN IA

MIKHAEL FAHMY I PROF DR A IN SHAMS UNIVERSITY

ARAB REP. OF EGYPT

MIKHELSON NIKOLAJ N DR PULKOVO OBSERVATORY 196140 LENINGRAD

U.S.S.R.

MILET BERNARD L DR $B$ P 252 F-C6007 NICE CEDEX

MILLAR THOMAS $J O R$

DEPT THEORET PHYSICS

OXFORD UNIV

1 KEBLE ROAD

OXFORD OXI 3NP

U.K.

MILLER JUHN C OR

MUICS

UNIV OF OXFORD

OXFORD

$\mathrm{U} \cdot \mathrm{K}$.

MILLIGAN J

ASTROPHYSICS BRANCH GODDARD SPACE FL CTR GREENEEL T MD 20771

U.S.A.

MILLMAN PETER M DR

HERZBERG INSTI TUTE

OF ASTROPHYSICS

NAT RES COUNC CANADA

OTTAWA ONT KIA ORG CANADA

MILNE K DR

DIV OF RADIOPHYSICS

O BOX 76

EPPING NSW 2121

AUSTRALIA

MILOVANOVIC VLADETA DR

INST ITUT ZA GEODE-

ZIJU BEOGRAD

BUL REVOLUCIJE 73

YUOOO BEOGRAD

MINNET HARRY C MR

CSIRO DIVISION OF

RADIOPHYS ICS

$P$ BOX 76

EPPING NSW 2121

AUSTRALIA
MIETELSKI JAN S DR

POLAND

MIHALAS DIMITRI DR

SACRAMENTO PEAK OBS

SUNSPOT NM 88349

U.S.A.

MIKHAIL JOSEPH SIDKY DR HELWAN OBSERVATORY

CARAB REP. OF EGYPT

MI LANO LEQPOLDO DR CAPODI MONTE ASTRONDMICAL OBSERVATORY VIA MOIARI ELLO 16 $1-80131$ NAPOLI

I TALY.

MILEY G K DR

POSTBUS 9513

$230^{\circ}$ RA LEIDEN

MILLER FREEMAN D PRCF UNI VERSITY MICHIGAN

DEPT OF ASTRONOMY

DENNISON BLDG

ANN ARBOR MI 48109

U.S.A.

MILLER JOSEPH S PROF

UNIVERSITY OF CALIF

UNIVERSITY QF CALIF

U.S.A.

MILLIKAN ALLAN G DR

RE SEARCH LABS B-59

EASTMAN KODAK CO

ROCHESTER NY 14650

U.S.A.

MILLS ALLAN A DR

DEPT OF ASTRONOMY

THE UNI VERSITY

LEICESTER LEI 7RH

U.K.

MILONE EUGENE F DR

PHYSICS DEPT

UNI VERSI TY

OF CALGARY

CALGARY ALTA T2N 1 N4 CANADA

MININ I $N$ DR

LENI NGRAD STATE UNI -

VERSITY ASTRONOMICAL

OBSERVATORY

I 99178 LENINGRAD

U.S.S.R.

MINTZ BLANCD MA B MR

CERRO TOLOLO INTER-

AMER I CAN OBS

LA SERENA

CA SEREN 


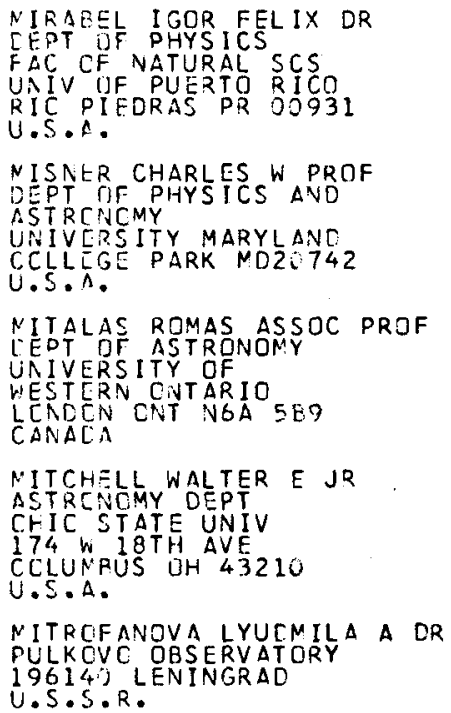

MIYANOTO MASANOR I DR TCKYC ASTRONOM ICAL

CESERVATORY

DSAWA MITAKA

TCKYO 181

JAPAN

NCCZKO JANUSZ DF

BCPCWIEC

63-120
PCLANO

MCELLENHOFF CLAUS DR

LANDESSTERNWARTE

KCENIGSTUHL

[-69ON HE IDELBERG 1

GERNANY, F.R.

MEESGAARD KRISTIAN P PH D RYEACEN

TCRRILC

CK-BวJO ODDER

LENNARK

NCFFETT THOMAS J PROF

DEPT OF PHYSICS

WEST LAFAYETTE

IN 47907

U.S.A.

MCISEEV I G DR

CRINEAN ASTRQPHYSI-

CAL ORSERVATORY USSR

ACAD OF SCI NAU

U.S.S.R.

NCLNAR MICHAEL R PROF

UAIVERS ITY OF TOLEDO

RITER OBSERVATORY

TCLEDO OH 43606

U.S.A.
MIRZOYAN L N DR PROF BYURAKAN ASTROPHYSI CAL OBSERVATORY

378433 ARMEN IA

U.S.S.R.

MISSANA MARCO DR $\checkmark$ IA BERGAMO

ITALY 20 OSNAGO COMO

MITCHELL GEORGE F DR

SAINT MARY S

UNIVERSITY

HAL IFAX NS B3H 3C 3

CANADA

MITIC LJUBISA A DR

OBSERVATOIRE

DE BEL GR ADE

VOLGINA 7

IIO50 RELGRADE

YUGOSLAVIA

MITTON SIMON DR

CAMBR IDGE UNIV PRESS

P D BOX 110

CAMBR IOGE CB2 3RL

U.K.

MIYAMOTO SIGINORI PROF

DEPT DF PHYSICS

MACHIKANEYAMA-CHO

MACHIKANEYAMA-CHO
TOYONAKA OSAKA 560

JAPAN

MODAL I SARMA B DR

6404 FAIRBORN TERR

NEW CARROLLTON

MD 20784

U.S.A.

MOELLER OLE PROF

INST OF ASTRONOMY

UN IVERSITY OF AARHUS

DK-8OOO AARHUS C

DENMARK MOFFAT ANTHONY F J OR UN IVER SI TE

DE MONTREAL

MONTREAL P \& H3C $3 J 7$ CANADA

MOGILEVSKI J EH I DR

INTR I IUTE ONETISM ÁND

IONOSPHERE

142092 AKADEMGORODOK

U.S.S.R.

MOLCHANOV A P PROF

LEN INGRAD STATE UNI -

VERSITY ASTRONOMICAL

OBSERVATORY

199178 LEN INGRAD

U.S.S.R.

MONAGHAN JOSEPH J DR

MATHEMATICS DEPT

MONASH UN I VERSITY CLAYTON VICT 3168 AUSTRALIA
MISCONI NEBIL YOUS IF DR

SPACE ASTRONOMY LAB

SUNY

EXECUTIVE PARK EAST

ALBANY NY 12203

U.S.A.

MISSANA NATALE PROF

VIA PUCCININ.3

I-10025 PINO

TORINESE

MI TCHELL R MR

C/OCICESE

AV CASTELUM NO 898

ENSENADA

BAJA CALIFORNIA

MEXICO

MI TRA A P DR

ASST DIRECTOR

NATI ONAL PHYS ICAL

LABORATORY

NEW DELHI

I NOIA

MIYAOI MASASI DR

SENG AWA 3-6-1 1

CHOFU-SHI 182

MIYAMOTO SYOTARO PROF DR KASAN OBSERVATORY

YAMA HIGASHIYAMA

KYOTO 607

JAPAN

MODISETTE JERRY L PROF

UNIVERSI TY

7502 FONDREN AVE

HOUSTON TX 77074

U.S.A.

MOEROI JK WILLY G DR

STERRENKUNDIG INST

KORTRIJKSE

STEENWEG 523

B-9DOO GENT

BELGIUM

MOFFET ALAN T PROF

OWENS VALLEY RADIO

OASERVATORY

CALTECH 102 24,125

U.S.A.

MOHLER ORREN C PROF

405 AWIXA RO

ANN ARBOR MI 48104

U.S.A.

MOLES MARIANO J DR

BINACEO HUESCA

SPAIN

MONFILS ANDRE G PROF INSTI TUT D-4200 COINTE-LIEGE BELG IUM 
NCANET GUY J DR

CPSERVATOIRE CE LYON AVENLE CHARLES ANCRE F-69230 ST GENIS LAVA

FRANCE

NCNIMERLE T DR

SECT DOASTROPHYSIQUE CEN SACLAY

5 INU 2

$F-91190$ GIF-S-YVETTE

FRANC?

FCCRE ELLIOTT P PROF JCIAT CES ERVAT ORY

F COMETARY RESEARCH

CANPUS STATION

SCCCRRC NM B780I

U.S. A.

NCCRE-SITTERLY CH MRS DR

PAT ICNAL BUREAU OF

STANEARES $151-00$

WASHINGTON DC 20234

U.S.A.

MCRAIIDC BRONO L CR

BUREAU D LONGI TUDES

77 AVE DENFERT

RCCHLREAU

F-75014 DARIS

FRANCE

NCRENO HUGO PROF

LEF UE $\triangle S T R O N O M I A$

UAIVERSIDAD OE CHILE

CASILLA 36-D

SANTIAGO

MCRGAi PETER CR

DIV CF NATFONAL

NAPPING

P C EOX 548

QUEANEEYAN NSW 2620

AUSTRALIA

NCRGULEFF NINA ING

INST D ASTROPHYS IQUE

98 B IS AD ARAGO

F-75CI 4 PARIS

FRANCE

NCRIYAMA FUMIO DR

TCKYC ASTRONOM ICAL

DESERVATORY

CSAWA NITAKA

TCKYC $1 \& 1$

JAPAN

NCRCZHENKO N N CR

MAIA ASTRON OES

UKRAINIAN ACAD OF SC

252127 KIEV

U.S.S.R.

MCRRIS S C DR

DCNINION ASTROPHYSI-

CAL CHSERVATORY

5071 W SAANICH RC RR7

VICTUKIA BC VBX $3 \times 3$

CANACA

NCRRISCN NANCY DUNLAP DR

UNIV OF TOLEDO

$28 J 1$ N EANCROFT ST

TCLEDC

U.S.A.
MONSIGNORI FOSSI BRUNA DR OSSERVATOR IO ARCETR I LARGO FERMI 5 A ITALY

MOOK DELD E PROF

DEPT OF PHYSICS AND

ASTRONOMY

DARTMOUTH COLLEGE
HANOVER NH 03755

U.S.A.

MOORE PATRICK DR OBE

FAR THINGS

39 WEST STREET
SELSEY SUSSEX

U.K.

MOOS HENRY WARREN DR

DEPT OF PHYSICS

JOHNS HOPK INS UNIV

U.S.A.

MORBEY CHR ISTOPHER L DOM IN ION ASTROPHYSI CAL OBSERVATORY

$5071 W$ SAANICH RD RR7

VICTOR IA BC V8X $3 \times 3$

CANADA

MORETON G E BALMORAL BCH NSW2088 AUSTRALIA

MORGAN THOMAS H DR HOUSTON BAPTIST

UN IVER S I TY

7502 FONDREN A VE
HOUSTON TX 77074

U.S.A.

MOR IMOTO MASAKI DR

TOKYO ASTRONOMICAL

ORSER VA TORY

OSAWA MITAKA

TOKYO 181

JAPAN

MOROZ V I PROF DR

SPACE RESEARCH

INST ITUTE

USSR ACADEMY OF SCI

117810 MOSCOW

MORR IS DAVIO DR

MPI FUER RADIOASTRON

AUF DEM HUEGEL 69

D-530O BONN 1

MORR ISON DAVID PROF NASE SL HEAOQUARTERS NASA HEADQUARTERS
WASHINGTON DC 20546 U.S.A.

MORR I SON PHILIP PROF PFYSICS MIT $6-205$
CAMBRIDGE MA 02139 U.S.A.
MONTES C DR

QBS DE NICE

F-06007 NICE CEDEX

FRANCE

MOORE D R DR

DEPT APPLIED MATHS

AND THEORETICAL PHYS

SILVER STREET

CAMBRIDGE CB3 $9 E W$

U. K.

MOORE RONALD $L$ DR

SOLAR ASTRONOMY

26433 CALIF INST TECHNOLOG

PALIF INST TECHNA CA 91125

U.S.A.

MORAN JAMES M DR

CENTER FOR

ASTROPHYSICS

6O GARDEN ST

CAMBRIDGE MA 02138

U.S.A.

MOREELS G DR

OBS DE BESANCON

41 BIS AVENUE DE

-250OO

FRANCE

MORGAN DAVID H DR

ROYAL DESERVATORY

BLACKFORD HILL

EDINBURGH EH9 $3 \mathrm{HJ}$

U. K.

MORGAN $W W$ PROF

YERKES ORSERVATORY

WILLIAMS BAY WI 53191

U.S.A.

MORISON IAN MR

NRAL

JODRELL BANK

CHESHIRE SKII 9DL

U. K.

MOROZHENKD A $Y$ DR

MAIN ASTRON OBS

UKRAINIAN ACAD OF SC

GOLOSEEVO

252127 KIEV

U.S.S.R.

MORR I S MARK ROOT DR

DEPT OF ASTRONOMY

COLUMBIA UNIV

NEW YORK NY 10027

U.S.A.

MORRI SON LESLIE Y

ROYAL GREENWICH

OBSERVATORY

HAI LSHAM

EAST SUSSEX BN27 IRP

U.K.

MORTON DONALD C DR

ANGLO AUSTRALIAN

OBSERVATORY

$P$ O BOX 296

EPPING NSW 2121 


\section{MCRTON G A DR
AIZT SKYCREST DRIVE \\ WALNUTT CREEK CA94595 \\ U.S.A. \\ MCTZ LLOYD PROF CCLUMBIA UNIVERS ITY PUPIN YCRK NY 10027 UEW Y.S. \\ NCUSSAS XENDPHON PH D 2 SKYLITSI STREET GREECE}

NEKCS ANT ONIN DR

DEPT OF ASTRONOMY CFARLES UNIVERSITY

150 OO PRAHA 5

MUELLER IVAN I PROF

CECEETIC SCIENCE

IO5 5 CIL AVE

CCLUMBUS OH 43210

U.S.A.

NUFSCN STUART LEE DR

ASTREIVMY DEPT

IRCIAMA UNIV

GLCONINGTON IN 47401

U.S.A.

MULDERS GERARD $F$ W 4517 EVERETT ST KENSINGTON MD 20795 U.S.A.

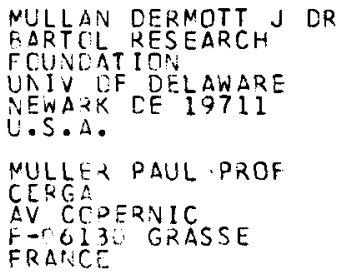

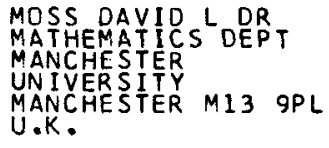

MOURADIAN ZADIG M DR

ORSERVATOIRE DE

PAR I S-MEUDON

F-92190 MEUDON

FR ANCE

MOUTSOULAS MICHAEL PRDF

ASTRONOMY DEPT

UN IVERSITY OF ATHENS

PANEP ISTIMIOPOLIS

ATHENS 621

GREECE

MUELLER EDITH A PROF

QBSERVATOIRE

DE GENEVE

CH-1290 SAUVERNY GE

SWITZERLAND

MUELLER R PROF DR

NEUBEUERER STRASSE I

GERMANY, F.R.

MUKAI SONOYO DR

K ANAZAWA INSTITUTE

OF TECHNOLOGY

ENKOJ IHONMACHI

KANAZAWA 921

JAPAN

MULHOLLAND J DERRAL DR

DEPT OF A STRONOMY

UN I VERSITY OF TEXAS

AUSTIN TX 78712

U.S.A.

MULLER A B DR

ESO SCLE ISSHE IMER STR 17

D-8O4E GARCHING BE I

MUENCHEN

GERMANY, F.R.

MULLER R DR

OBS DU PIC-DU-MIDI

F-65200 BAGNERES-DE-

BIGORRE

FRANCE

MURDIN PAUL G DR

ROYAL GREENWICH

OBSER VATORY

HAILSHAN

EAST SUSSEX BN27 IRP

U.K.

MURRAY C ANDREW

ROYAL GREENWICH

OASERVATORY

HAIL SHAM

EAST SUSSEX BN27 IRP

U.K.

MUSEN PETER OR

NASA GSFC

GREENBELTT MD 20801

$U \cdot S \cdot A$.
MOTTA SANTO OR

CITTA UNIVERSITARI A

ITÁLY

MOUSCHOVIAS TELEMACHOS $\mathrm{CH}$

DEPT OF ASTRONOMY

UNI VERS ITY I LLINOIS

URBANA IL 61801

U. S.A.

MOVAHED REZA DR

P O BOX

IRAN

MUELLER HELMUT O PROF

HERZOGENMUEHLESTR 4

CHIBOSI ZUERICH

MUENCH GUIDO PROF

MPI FUER ASTRONOMIE

KOENIGSTUHL

D-6900 HEI DELBERG 1

MUKAI TADASHI DR

KANA Z AWA INSTI TUTE

OF TECHNOLOGY

ENKOJ IHONMACHI

KANAZAWA 921

JAPAN

MULLALY RICHARD F DR

SCHOOL OF ELECTRICAL

ENG INEER I NG

UNIV OF SYDNEY

SYDNEY NSW 2006

AUSTRALIA

MULLER C A PROF ING

OD INKSVELD 8

DELDEN

THE NETHERLANDS

MUMFORD GEORGE $S$ PROF

BALLQU HALL

TUF TS UNIVERSITY

MEDFORD MA O2155

U.S.A.

MURDOCH HUGH S DR

UNI VERSITY OF SYDNEY

SYDNEY 2006

AUSTRALIA

MURRAY JOHN B DR

UNIV OF LONDON

OBSERVATORY

MILL HILL PARK

LONDON NWT $20 S$

U. K.

MUSMAN STEVEN DR

SACRAMENTO PEAK

OQBSERVATORY

SUNSPOT NM 88349

U.S.A. 


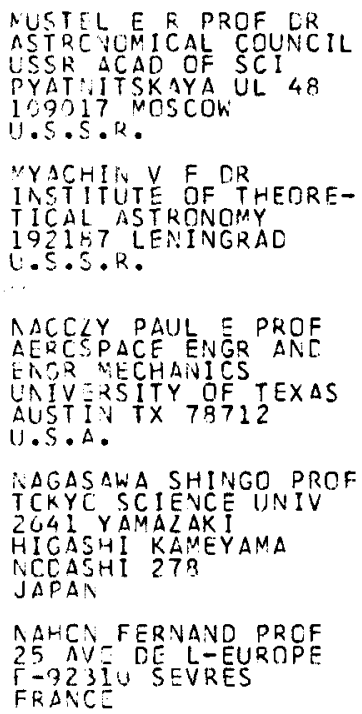

NANCY KASHINATT QR

KCYAL CBSERVATORY ECINEURCH

AAUNOV VITALIJ A DR PULKCVC OBSERVATORY 19614.2 LENINGRAD U.S.S.R.
MUTSCHLECNER J PAUL DR

SWAIN HALL W

INDIANA UN IVER SITY

BLOOMINGTON IN 47405

U.S.A.

MYERS PHILIP C PROF

PHYSICS DEPT

MIT

BLDG 26 RM 349

CAMBR IDGE MA 02139

U.S.A.

NADOLSCHI $V$ PROF DR

COM TARDEOANI

BACAU

ROUMANIA

NAGIRNER DMITRIJ I DR

LENINGRAD UN IVER SITY

ASTRONOMICAL OBS

199178 LENINGRAD

U.S.S.R.

NAKAGAWA NAQYA DR

UNIV OF ELECTRO-

COMMUNICATIONS

CHOFU-SHI

TOKYO 181

JAPAN

NAKANO SABLRO DR

KCBINATO 1-21-7

BUNKYO KU

TUK YO 112

JAPAN

NAKAZAWA KIYOSHI OR

DEPT OF PHYSICS

KYOTO UN I VER SI TY

KITASHIRAKAWA OIWAKE SAKYOKU KYOTO 606 $J A P A N$

NAPIER WILLIAM M DR

ROYAL OB SERVATORY

BLACKFORD HILL

EDINBURGH EH9 $3 \mathrm{HJ}$

U.K.

NARAYANA J V

REG METEOROLOGICAL

OFF ICE

4 COLLECE ROAD

MARAS 600006

INDIA

NARITA SHINLI DR DOSHISHA UNIVERSITY JYOTO 602

JAP AN

NATHER R EDWARD

UEPT OF ASTRONOMY

AUSTIN TX 78712

U.S.A.

NAWAR SAMIR DR HELWAN DBSERVATORY HELWAN

$A R A B$ REP . OF EGYPT
MUZZIO JUAN C DR

OBSERVATORIO

ASTRONOMICO

PASEO DEL BDSQUE

1900 LA PLA

ARGENTINA

MYERSCQUGH VALERIE P DR

DEPT OF APPLIED MATT

QUEEN MARY COLLEGE

MILE END ROAD

LONDON EI 4 NS

NADYOZHIN D K DR

INST THEOR EXP PHYS

117259 MOSCOW

U.S.S.R.

NAGNIBEDA VALERY G DR

LENI NGRAD UNI VERS ITY

ASTRONOMICAL

199178 LENI NGRAO

U.S.S.R.

NAKAGAWA YOSHINARI DR

HIGH ALTI TUDE

OBSERVATORY

$P$ O BOX 3000

BOULDER CO 80307

U.S.A.

NAKANO TAKENORI DR

DEPT OF PHYSICS

KYOTO UNIVERSITY

KI TASHIRAKAWA OIWAKE

SAKYOKU KYOTO 606

JAPAN

NAMBA OSAMU DR

ASTRONOMICAL INST

ZONNENBURG

$3512 \mathrm{NL}$ UTRECHT

THE NETHERLANDS

NAQVI A M DR

6274 DEL ROSA AVE

APT 11

S RERNARDINO CA92404

U.S.A.

NARI AI HIOEICH!

RESEARCH INST FOR THEORETICAL PHYSICS 725 TAKEHARA JAPAN

NARLIKAR JAYANT $Y$ PROF KING S CQLLEGE

CAMBRIDGE

U.K.

NATTA ANTONELLA DR

CNR A STROF I I I CA

SPAZIALE C P 67 .

ROMA

I TALY

NECKEL HEINZ DR

HAMBURGER STERNWARTE $D-2050$ HAMBURG 80 GERMANY, F.R. 


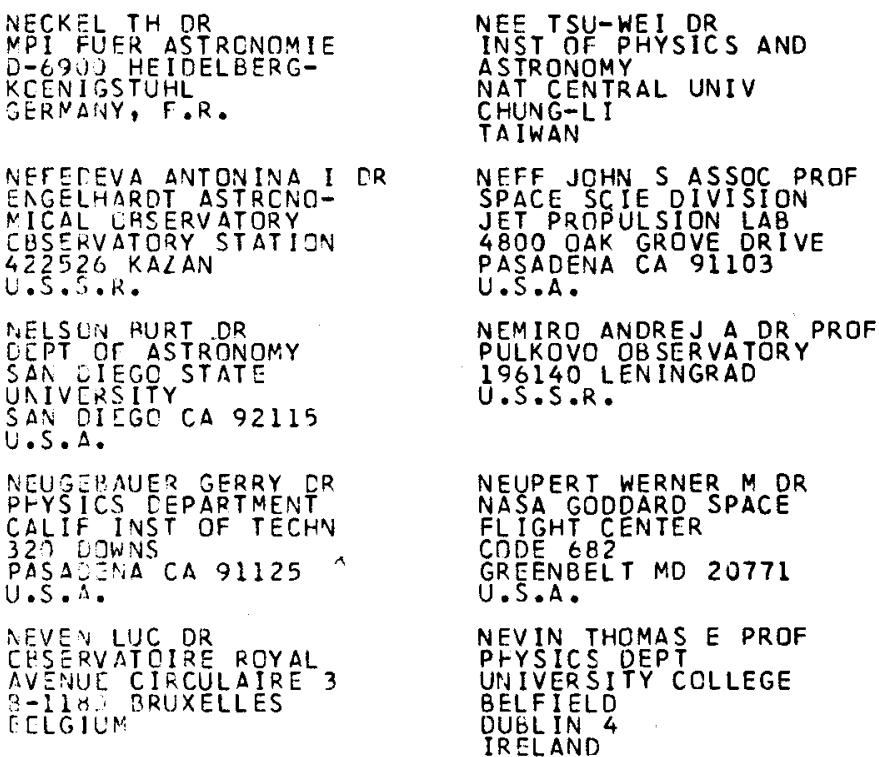

NEE TSU-WEI DR

INST OFF PHYSICS AND A STRONOMY

NAT CENTRAL UNIV

CHUNG-LI

TAIWAN

NEFF JOHN S ASSOC PROF

SPACE SCIE DIVISION

JET PROPULSION LAB

4800 OAK GROVE DR I VE

PASADENA CA 91103

U.S.A.

NEMIRD ANDREJ A DR PROF PULKOVO OB SERVATORY 196140 LENINGRAD

U.S.S.R.

NEUPERT WERNER $M$ DR NASA GODOARO SPACE FL IGHT CENTER

GREENBELT MD 20771

U.S.A.

NEVIN THOMAS E PROF PFYSICS DEPT

UN IVERSI TY COLLEGE

BELFIELD

OUGLIN 4

NICOLOV N S QR

UAIVERSITE DE SOFIA

SCFI

EULGÁIA

NISNELA VIRPI S QR CALLE 51 ESO 11 DA ARGENTINA

NIKITINA A DR LEA USRAC STATE UVICESERVATCRY 139173 LENINGRAD 1:. S.S.R.
NEWMAN MICHAEL JOHN DR

T-DOT MS 288

LOS ALAMOS SC LAB

LOS ALAMOS NM 87545

U.S.A.

NEY EDWARD P PROF

DEPT OF ASTRONOMY

MINNEAPOL IS MN 55455

U.S.A.

NIARCHOS PANAYIOTIS PH D DEPT OF ASTRONOMY UNIV OF A THENS

PANEPISTIMIOPOLI IS

ATHENS 621

GREECE.

NICOLET MARCEL PROF

UN IVERSITE

DE BRUXELLES

30 AVE DEN DOORN

B- 1180 BRUXELLES

BELGIUM

N ICOLSON GEORGE D DR

RADIO ASTRONOMY

OBSERVATORY

P O BOX 3718

JOHANNESBURG 2CCO

SOUTH AFRICA.

NIEUWENHUIJZEN HANS DR ASTRONOMICAL INST ZONNENBURG 2

UTRECHT

THE NETHERLANDS

NIKOLOFF IVAN DR PERTH OBSERVATORY BICKLEY W'A 6076 AUSTRALIA
UNIV OF MINNESOTA
NEE MAN YUVAL PROF DEPT DF PHYSICS AND ASTRONOMY

TEL AVIV UNIVERSITY

TEL AVIV

I SRAE L

NELSON ALISTAIR H DR AND ASTRONOMY UNIV COLLEGE CARDIFF

U. K.

NESS NORMAN F DR NASA GSFC CODE 690 U. S.A.

NEUZIL LUDEK DR

ASTRONOMICAL INST CZECH ACAD SC IENCES

OB SER VATORY

251-65 ONDREJOV

CZECHOSLOVAKIA

NEWELL EDWARD B DR

MT STROMLO AND

SIDING SPRING OBS

PRIVATE BAG

WODEN A A C

NEWSON GERALD H ASST PROF

ASTRONOMY OEPT

DHIO STATE UNIV

$174 \mathrm{~W} 18 \mathrm{TH}$ AVE

COLUMBUS OH 43210

U.S.A.

NEYMAN JERRY PROF

STATISTICAL LAB

UNIV OF CALI FORNIA

367 EVANS HALL

BERKELEY CA $\$ 4720$

U.S.A.

NICHOLLS RALPH W PROF

CRESS DEPT OF PHYS

YORK UNIVERSITY

470 ? KEELE ST

DOWNSVIEW ONT M3JIP3

CANADA

NICOLINI T PROF

VIA E NICQLAROI

COLLI AMINE

I OLDI 31 NAPOLI

221

NIELL ARTHUR E DR

FHL

4800 DAK GROVE DR

PASADENA CA 91103

U.S.A.

NIIMI HIDEYUKI DR

FACULTY EÑGINEERING

UNIVERSI TY OF KYOTO

YOSH I OAHONMACHI

SAKYOKU KYOTO 606 JAPAN

NIKOLOV ANDREJ DR

$P O B O X \quad 402$

RULGARIA 
AIKCLSKY G M PROF DR INST ITUTE OF TERREI CNCSPHERE

142192 AKADEMGORODOK

U.S.S.R.

NILSSON CARL CR

SNITHSCNIAN ASTRO-

PHYS ICS OBSERVATORY

CO GAREEN STREET

U.S.A.

NISHIDA MINORU PROF

DEPT OF PHYS ICS

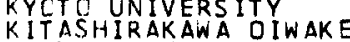

SAKYCKU KYOTO 606 $J A P \triangle N$

NISSEN POUL E PROF

IAST OF ASTRONOMY

UAIVERSITY OF AARHUS

LANGELANDSKADE

CK-8000 AARHUS C

EEIVNARK

NCEL FERNANDO

CPTC DERASTRONOMIA

UNIVERSIDAD DE CHILE

CASILLA 36D

CHILE

NCLLEL G CR

IAST D. ASTROPHYSIQUE

98 R IS BD ARAGO

F-75014 PARIS

FRANCE

VCRCH H LENNART OR

STCCKHCLM

$S-13300$

SALTS JOERADEN

SWEOEN

YCRL I NU WILHELM CR

SVANFGATAN 16

$5-222 \quad 24$ LUND

SWECEN

NCRRIS JOHN DR

OESERVATORY

WCDEN PO ACT 26:6

$\triangle U S T R A L I A$

NCTNI P DR

ZNTRI INST ASTROPHYSIK

STERNW BABELSBERC

RCSA LUXEMBURGST 174

CER- 1502 POTSCAM

G.0.k.

NCVIKOV I D. DR

SPACE RESEARH

USSR ACADEMY OF SCI

$117819 \mathrm{M}$

NCYES ROEERT W PROF

CTR F ASTROPHYSICS

OO GARDEN ST

CANORIEGE MA 02138

U.S.A.
NIKONOV V B DR

CRIMEAN ASTROPHYSI-

CAL OBSERVATORY USSR

334413 CR IMEA

$U . S \cdot S \cdot R$.

NIN INGER $H H$

BOX 420

SEDONA AZ 86336

U.S.A.

NISHIMURA JUN DR

INST SPACE AERON SC

KOMABA MEGURO-KU

TOKYO

JAPAN

NOB IL I L DR

OSS ASTRONOMICO

$\checkmark I C O L O$ DELL OSSERVA-

TOR IO

PADOVA

ITALY

NOELS ARLETTE UR

50 AVENUE DE LA PAIX

BOITE 063

$B-4030$ GR IVEGNEE

BELGIUM

NOMOTO KENICHI

IBARAK I UNIVERSITY

BUNKYO MITO 310

JAPAN

NORDLUND AKE DR

NORDITA

BLEGDAMSVEJ 17

DK - 2100 COPENHAGEN O DENMARK

NORLUND N E PROF

MALMOGADE 6

DK-21OO COPENHAGEN O

DENMARK

NORTH JOHN D PROF

28 CHALFONT ROAD

OXFORD

U.K.

NOTUKI MASAO DR

4-36-7 NAR I TAHIGASHI

SUG INAM I-KU

TOKYO 166

JAPAN

NOVIKOV SERGEJ B DR

STERNBERG STATE

ASTRONOMICAL INST

II 7234 MOSCDW

U.S.S.R.

NUSSBAUMER HARRY DR ATOMIC AND ASTROPHYS GROUP ETHZ

HUT TENSTR 34

CH- 8092 ZUER ICH
NILSON PETER DR

OB SERVATORY

BOX 515

S-751 20 UPPSALA
SWEDEN

NISHI KEIZO OR

TOKYO ASTRONOMICAL

OBSERVATORY

OSAWA MITAKA

TOKYO 181

JAPAN

NI SHIMURA SHIRO DR

TOKYO ASTRONOMICAL

OBSERVATORY

OSAWA MITAKA

TOKYO 181

JAPAN

NOCI GI ANC ARLO PROF

OSSERVATORI O ARCETRI

I 50125 FIRENZE

NOERDLINGER PETER D.PROF.

NASA AMES RES CTR

245-3

FLD CA 94035

U.S.A.

NOONAN THOMAS W PROF

PHYSICS DEPT

SUNY

BRDCKPORT NY 14420

U.S.A.

NOROSTROM BIRGITTA DR

COPENHAGEN

OBSERVATORY

BRORFELDE

DK -4340 TOLLOSE

DENMARK

NORMAN COLIN DR

HUYGENS LAB

STERREWACHT

2300 RA LEIDEN

THE NETHERLANDS

NOSKOV BORIS N DR

STERNBERG STATE

117234 MOSCOW

U. S. S.R.

NOVICK ROBERT

COLUMBI A UNIVERSITY

538 W 120 ST

NEW YORK NY 10027

U. S.A.

NOVOSELOV V S PROF DR

LENINGRAD STATE UNI-

OBSERVATORY

I99178 LENINGRAD

U.S.S.R.

O'CONNELL D J K J J DR

ITALY 
C'CCANELL ROBERT F PROF LCUISIAVA STATE

PATCN ROUGE LA 70803

U.S. 4 .

C'DELL STEPHEN L

PHIYSICS DEPT

$\checkmark P$ I ANO $S U$

ELACKSPURG VA 24061

U.S.A.

CEKEEFE JOHN A CR

CENT CR CODF 680

GREENRELT MD 20771

U.S.A.

CSULLIVAP JOHN CAVID DR RADI OS TERREW ACHT

FESTEUS 2

799 A A OWINGELOO

THE NETHERLANDS

CEI SHINYA PROF

FAC OF GENERAL ED.

UAIV OF IOKYO

TCKYE 153

JAPAN

CCCHIOAERO FRANCO PROF

CNR ASTROF IS ICA

SPAZIALE C P 67
$1-00944$ FRASCAT I

RONA

OCGERS GRAHAM J DR

CCNINICN ASTRO OBS

NAT RES COUNC CANADA

5071 WEST SAANICH RD

VICTCRIA BC V8X $3 \times 3$

CANACA

CESTTRWINTER CLAUS

CCNMANDER

NAVAL SURFACE WEA-

PCNS CENTER DK WO

CAHLGREN VA 22448

U.S.A.

OHKI KENICHIRO DR

TCKYC ASTRONOMICAL

OBSERVATORY

CSAWA

NITAKA TCKYO 181

JAPAN

CJA TARMO PROF

KVISTABERG

OESERVATORY

SWECEN I BRD

CKE J BEVERLY PRCF

HALE OBSERVATORIES

CALIFORNIA INSTITUTE

CF TECHNDLOGY

PASADENA CA 91125

U.S.A.

CKUDA HARUYUKI CR PROF

DEPT OF PHYSICS

KYCTO UNIVERSITY

KITASHIRAKAWA SAKYO

KYCTE 606
O'CONNELL ROBERT WEST DR

ASTRO DEPT UNIV VA

PO BOX 3818 UNIV STA

CHARLOT TE SVILLE

VA 22903

U.S.A.

O HANDLEY DOUGLAS A DR

JET PROPULSION

$L A B O R A T O R Y$

BLDG 169422

PASADENA CA 91103

U.S.A.

DLEARY BRIAN T

PRINCETON UNIVERSITY

PRINCETON NJ OB540

U.S.A.

QRASHEV SAKEN O DR

ASTROPHYSICAL

INSTITUTE

480068 ALMA-ATA

U.S.S.R.

OBREGON DIAZ OCTAVIO J DR

UAM I T TAPALAPA

PD BDX 55534

MEXICO I3 DF

MEXICO

ODA MINORU PROF

INST SPACE AERON SC

UN IVERSITY OF TOKYO

KOMABA MEGURO KU

TOKYO 153

JAPAN

DEHMAN YNGVE PROF

THULEHEM 53

SW 22367 LUND

OETKEN L DR

ZNTRINST ASTROPHYSIK ASTROPHY OBSER VATORI

TELEGRAFENRERG

DDR- 15 POTSDAM

$G \cdot D \cdot R$.

QHR ING GEORGE PROF DEPT OF GEOPHYSICS PLANETARY SCIENCES

TEL AVIV

I SRAEL

OKA TAKESHI DR

HERZBERG INSTI TUTE

OF ASTROPHYSICS

NRCC

QTTAWA ONT KIA ORG

OK I TOSHIO PROF

FACULTY OF EDUCATION

FUKUSHIMA UNIVERSITY

HAMADACHO

FUKUSHIMA 960

JAPAN

OKUDA TOYOZO DR

5-11-9 HANAZONO

CHIBA 281

JAPAN
Q'CQNNOR SEAMUS L DR

PHYSICS DEPY

UNI VERSI TY COLLEGE

DUBLIN 4

I RELAND

Q'HORA NATHY P J

ROYAL GREENWICH

DESERVATORY

HAILSHAM

EAST SUSSEX BN27, LRP

U.K.

O'MARA BERNARD J PROF

DEPT OF PHYSICS

UNIV OF QUEENSLAND

ST LUCIA

BRI SBANE QLD 4067

ORERLERCHNER DOROTHEA DR

UNIV-STERNWARTE

TUERKENSCHANZSTR 17

$A-1180$ WIEN

AUSTRIA

OBRIDKO VLADIMIR A DR

I ZMI RAN

142092 MOSCDW REGION

U.S.S.R.

ODFLL CHARLES R DR

HUNTSVILLE

U.S.A.

OEMLER AUGUSTUS JR DR

YALE UNIV OBS

$80 \times 2023$

YALE STATION

NEW HAVEN CT 06520

U.S.A.

OGORODNIKOY KYRILL P PROF

DEPT OF ASTRONOMY

199164 LENINGRAD

U.S.S.R.

OHYAMA NOBORU PROF

FAC OF ENG INEERING

SHIZUOKA UNIVERS ITY

3 CHOME JYOHOKU

HAMAMATSU 432

JAPAN

OKAMOTO ISAO OR

INTERNAT I ONAL LATITU

DE OBSERVATORY

HŌSHIGAOKA MIZUSAWA

I WATE 023

JAPAN

OKOYE SAMUEL E PROF

DEPT OF PHYSICS

NIGERIA

NSUKKA

NIGERIA

OLEAK H DR

ZNTRINST ASTROPHYSIK

STERNW BABELSBERG

ROSA LUXEMBURGST 17 A

DDR- 150

$G \cdot D \cdot R$. 


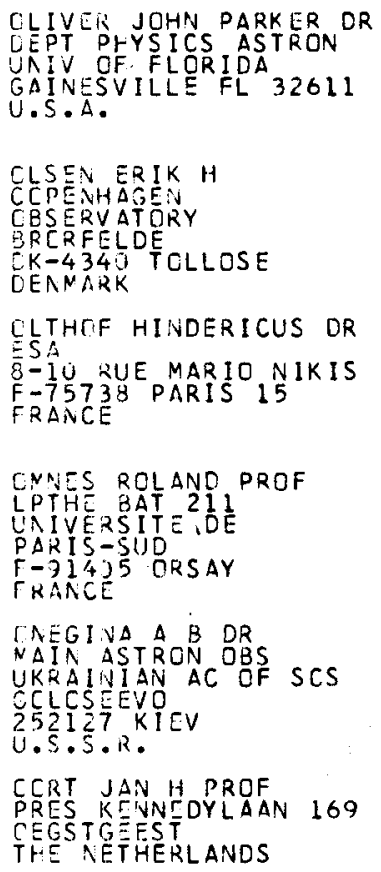

\footnotetext{
CPIK ERNST J DR ARNAGH OBSERVATORY ARMACH BT 1 I $90 \mathrm{G}$ U.K.
}

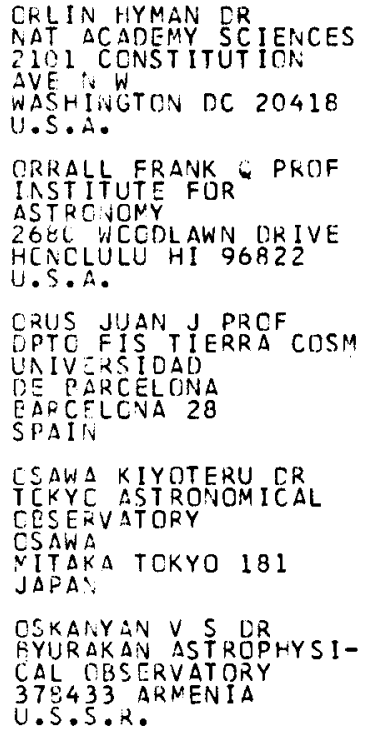

\begin{tabular}{|c|c|}
\hline $\begin{array}{l}\text { QLLONGREN A DR } \\
\text { DEPT OF MATHEMATICS } \\
\text { WASSENAAR SEWEG } 80 \\
\text { POSTBOX } 9512 \\
2300 \text { RA LE IDEN } \\
\text { THE NETHERLANDS }\end{array}$ & $\begin{array}{l}\text { QLOFSSON S GOERAN DR } \\
\text { STOC KHOLM } \\
\text { OBSERVATORY } \\
\text { S-I } 3 \text { O OO } \\
\text { SALTS JOEBADEN } \\
\text { SWEDEN }\end{array}$ \\
\hline $\begin{array}{l}\text { OLSEN KENNETH H DR } \\
\text { LOS ALAMOS SCIENTI- } \\
\text { FIC LABORATORY } \\
\text { BOX } 1663 \text { MS } 676 \\
\text { LOS ALAMOS NM } 87544 \\
\text { U.S.A. }\end{array}$ & $\begin{array}{l}\text { OLSON EDWARD C PROF } \\
\text { OESERVATORY } \\
\text { UNIVERSITY OF } \\
\text { ILLINOIS } \\
\text { URBANA IL } 62801 \\
\text { U.S.A. }\end{array}$ \\
\hline $\begin{array}{l}\text { OMAROV TUKEN B PROF } \\
\text { ASTROPHYSICAL } \\
\text { INSTITUTE ICA } \\
480068 \text { ALMA ATA } \\
\text { U.S.S.R. }\end{array}$ & $\begin{array}{l}\text { QMER GUY C JR PROF } \\
1 \text { OBD SW IITH TERRACE } \\
\text { GAINE SVILLE FL } 3260 \mathrm{I} \\
\text { U.S. A. }\end{array}$ \\
\hline $\begin{array}{l}\text { OMONT ALA IN PROF } \\
\text { OBSERVATOIRE } \\
\text { DE MEUDON } \\
\text { F-9219O MEUDON } \\
\text { FRANCE }\end{array}$ & $\begin{array}{l}\text { ONDERLICKA BEDRICH DR } \\
\text { DEPT OF ASTRONOMY } \\
\text { PURKYNE UNI VERSITY } \\
\text { KOTLARSKA } 2 \\
61137 \text { BRNO } \\
\text { CZECHOSLOVAKIA }\end{array}$ \\
\hline $\begin{array}{l}\text { ONO YDRO PROF } \\
\text { DEPT OF PHYSICS } \\
\text { UN IVER SITY HOKKAIDO } \\
\text { KI I AHACH J JYO NISHI } 8 \\
\text { SAPPORO HOKKAIOO } 063 \\
\text { JAPAN }\end{array}$ & $\begin{array}{l}\text { OOE MASATSUGU DR } \\
\text { INTERNATIONAL LATITU } \\
\text { DE OBSERVATORY } \\
\text { HOSHIGAOKA MIZUSAWA } \\
\text { IWATE OZ3 } \\
\text { JAPAN }\end{array}$ \\
\hline $\begin{array}{l}\text { OPALSKI WIESLAW PROF } \\
\text { ELEK TORALNA } 4-6 \text { M4 } \\
\text { OO-I39 WARSAW } \\
\text { POLAND }\end{array}$ & $\begin{array}{l}\text { QPHER REUVEN PROF } \\
\text { PHYSICS DEPT } \\
\text { TECHNION-ISRAEL INST } \\
\text { OF TECHNOLOGY } \\
\text { HAIFA } 32000 \\
\text { ISRAEL }\end{array}$ \\
\hline $\begin{array}{l}\text { OPOLSK I ANTONI PROF } \\
\text { ASTRONOMICAL } \\
\text { OBSERVATORY } \\
\text { KOPERNIKA II } \\
5 I-622 \text { WROCLAW } \\
\text { POLAND }\end{array}$ & $\begin{array}{l}\text { QPROIU TIBERIU DR } \\
\text { STR BUCIUM } 25 \text { BLOC } \\
\text { R-5 SC I ET I I AP } 10 \\
3400 \text { CLUJ-NAPOCA } \\
\text { ROUMANIA }\end{array}$ \\
\hline $\begin{array}{l}\text { ORLOV A A DR } \\
\text { STERNBERG STATE AS- } \\
\text { TRONOM ICAL INSTITUTE } \\
\text { IIT234 MOSCOW } \\
\text { U.S.S.R. }\end{array}$ & $\begin{array}{l}\text { ORLQV MIKHAIL DR } \\
\text { MAIN ASIRONOMICAL OB } \\
252 I 27 \text { KIEV } \\
\text { U.S.S.R. }\end{array}$ \\
\hline $\begin{array}{l}\text { ORTE ALBERTO } \\
\text { IST Y Y OBSERVATORIO } \\
\text { DE MAR INA } \\
\text { SAN FERNANDO CADIZ } \\
\text { SPAIN }\end{array}$ & $\begin{array}{l}\text { ORTON GLENN S DR } \\
\text { MS I } 33 \text { B }-365 \\
\text { JPL. } \\
480 \text { OAK GROVE ORIVE } \\
\text { PASADENA CA 9IIO3 } \\
\text { U.S.A. }\end{array}$ \\
\hline $\begin{array}{l}\text { QSAKI TORU DR } \\
\text { RYUKOKU UNIVERSITY } \\
\text { FUKAKUSA TSUKAMOTO } \\
\text { FUSHIMIKU KYOTD } 612 \\
\text { JAPAN }\end{array}$ & $\begin{array}{l}\text { OSAKI YOJI DR } \\
\text { OEPT OF ASTRONOMY } \\
\text { UNIV OF TOKYD } \\
\text { YAYOI BUNKYO } \\
\text { TOKYO } 113 \\
\text { JAPAN }\end{array}$ \\
\hline $\begin{array}{l}\text { OSBORN WAYNE DR } \\
\text { PYYSICS DEPT } \\
\text { CENTRAL MICHIGAN } \\
\text { UNIVERSITY } \\
\text { MT PLEASANT MI } 48859 \\
\text { U.S.A. }\end{array}$ & $\begin{array}{l}\text { OSBORNE JOHN L OR } \\
\text { DEPT OF PHYSICS } \\
\text { UNIV OF DURHAM } \\
\text { SOUTH ROAD } \\
\text { DURHAM DHI } 3 L E \\
\text { U.K. }\end{array}$ \\
\hline $\begin{array}{l}\text { OSMAN ANAS MOHAMED DR } \\
\text { HELWAN OBSERVATORY } \\
\text { HELWAN } \\
\text { ARAB REP. OF EGYPT }\end{array}$ & $\begin{array}{l}\text { OSMER PATRICK S DR } \\
\text { CERRO TOLOLO INTER- } \\
\text { AMERICAN OBSERVATORY } \\
\text { CASILLA } 63 \text { D } \\
\text { LA SERENA } \\
\text { CHILE }\end{array}$ \\
\hline
\end{tabular}


CSCRIC JOSE J S P DR

UNIV UC PURTO

NCNTE LA VIRGEM

VILA NCVA DE GAIA

PCRTUGAL

CSTRIKER JEREMIAH P PROF

PRINCETCN UNIVERSITY

CBSERVATORY

PEYTCN HALL

U.S.A.

QVENCEN MICHAEL W PROF AND ASTRCNOMY

UAIVERSITY OF B

VANCEIJVER BC VET IWS

CAIVACA

CWEN TCBIAS C PRUF

DEPT OF EARTH AND

SPACE SCIENCES

STATE UTIVERSITY NY

STCNY BRCOK NY 11794

U.S.A.

CZEMRE KAMURAN COCENT DR

UNIVERSITY

CBSERVATORY

UAIVERSIT:

I T T $\triangle$ N $3 \cup L$

TURKEY

PAAL GYORGY DK

KCNKCLY OBSERVATORY

$\triangle[X 67$

1525 BUCAPEST

HUUNGARY

PACINI FRANCO PROF

ISTITUTO ASTRONOMIA

LARGO E FERMI 5

ITALY

PALRIELLI

LAH OI RAOIOASTRON

IA IRNERIO 46

I-40I26 BOLOGNA

ITALY

PAGEL RERVARDE J PROF

RCYAL GREENWICH

CBSERVATDRY

HAILSHAM

EAST SUSSEX BN27 IRP

U.K.

PALNEIRA RICARDO A R DR

INPE CP 515 D CAMPOS

$S A C$ PAULO

PRAZIL

PALUMSO GIORGIO G C DR

VIA DE EASTAGNOL I 1

I -4 U 1 . Y O BOLOGNA

ITALY

PANDE MAHESH CHANCRA DR UP STATE OBSERVATORY

NANCRA PEAK

NAINI TAL 263129

INDIA
DSTER LUDWIG F PROF DR JILA

UNIV OF COLORADO

BOULDER CO 80309

U.S.A.

OTERMA LIISI PROF

SIRKKALANKATU 31

SF-20700 TURKU

FINLAND

OWAKI NAOAKI DR

TOKYO GAKUGE I

UN IVER S I TY

NUKUIK I TAMACHI

KOGANEI TOKYO 184

JAPAN

OWREN LEIF DR

FYSISK INSTITUTT

UNIVERS ITETET BERGEN

ALLEGATEN 5355

$N-5014$ RERGEN U

NORWAY

DZERNOY LEONID M PROF

PUYSICAL INSTI TUTE

SC IENCE

I17924 MOSCOW

U.S.S.R.

PACHNER JAROSLAV PROF DEPT OF PHYSICS AND

ASTRONOMY

UNIVERSITY OF REGINA

REGINA SK S4S OA2

CANADA

PACZYNSKI B PROF

COPPERNICUS ASTRONOMI

- CAL CENTER

UL BARTYCKA

POLAND

PAGE CLIVE G DR

UNIV OF LEICESTER

U.K.

PAL ARPAD PROF DR

STR RAKOCZI 72

3400 CLUJ NAPOCA

ROUMAN IA

PALMER HENRY $P$ DR

NRAL

JODRELL BANK

CHESHIRE SKII $90 \mathrm{~L}$

U.K.

PAMJATN IIKH A A DR ASTONOMICAL COUNCIL USSR ACAD OF SC S 109017 MOSCDW

U.S.S.R.

PAINEK ROBERT J DR

DEPT OF ASTRONOMY

525 DAVEY LAB

UNIV OF PENNA

UNIV PK PA 16802

U.S.A.
OSTERBROCK DONALD E PROF LICK OBSERVATORY

UNI VER I TY

OF CALIFORNIA

SANTA CRUZ CA 95064

U.S.A.

OTTELET I J OR

TOPHYS I QUE B-420O COINTE OUGREE BELGIUM

OWEN FRAZER NELSON DR NATI ONAL RADIO ASTR OBSER VATORY

EDGEMONT RD

CHARLOTTESVL VA22901

U.S.A.

OXENIUS JOACHIM DR

UNI V L IBRE BRUXELLES

CP 231

CAMPÚS PLAINE ULB

B- 1050 BRUSSELS

BELGIUM

OZSVATH I PROF

EARTH AND PLANET SCS

P O BOX 30365

DALLAS TX 75230

U.S.A.

PACHOLCZYK ANDRZEJ G PROF

STEWARD OBSERVATORY

UNI VERSITY ARIZONA

TUC SON AZ 85721

U.S.A.

PADEVET VLADIMIR DR

ASTRONOMICAL INST

CZECH ACADEMY SC

OSI 65 ONDREJOV

CZECHOSLOVAKIA

PAGE THORNTON $L$ DR

18639 POINT LOOKOUT

DRIVE

HOUSTON TX 77058

U.S.A.

PALLAVICINI ROBERTO DR

OSSERVATOR IO ARCETRI

LARGO FERMI 5

I TALY 5 FI 25 IRENZE

PALMER PATRICK E PROF

DEPT OF ASTRONOMY

UNI VERSITY CHICAGO

CHICAGO IL 60637

U.S.A.

PANAGI A NIND DR

$\angle A B O R A T O R I O$ DI

RADI OA STRONOMI A

VIA IRNERID 46

$I=40126$ BOLOGNA

ITALY

PANKONIN VERNON LEE DR

DIV ASTRON SCIENCES

NAT SCI I NCE FOUND

$1800 \mathrm{G} S T$ SW

WASHI NG TON DC 20550

U. S.A. 
PANCV K DR

DEPT OF ASTRONOMY

QULGARIAN AC OF SCS IOJO SOFIA

BULGARIA

PAPALIOLIOS C DR

SNITHSCNIAN ASTRO-

PHYSICAL OBS

CANBRIDGE MA 02138

U.S.A.

PACUET PAUL EG LR

CE RELGIOUE

$A V E$ CIRCULAIRE 3

B-IISU BRUXELLES

EELGIUN

PARIJSKIJ $N N$ DR

IASTIIUTE OF PHYSICS

UT TH= EARTH

USSR ACAD DF SCI

123810 MOS COW

U.S.S.R.

PAEKER EUGEVE N

UNIV UF CHICAGO

333 E 56 TH ST

CH. ICAOC IL 60637

U.S.A.

PARKINSON T DR

KITT PEAK NAT OAS

950 CHERRY AVE

TUCSCW AZ 85726

U.S. 1 .

PARSAMYAN E S CR PYURAKAN ASTROPHYS ICAL CBSERVATORY 378433 ARMENIA

U.S.S.R.

PARTKIUGE ROBERT B PROF HAVERFCRO COLLEGE

HAVEKF

PASCU CAN OR

US NAVAL UESERVATORY WASHINGTON DC 20390

U.S.A.

PATEKINO LUCIO CR

CSSEKVATORIO

ASTRCFISICO

CITTA UNIVERSITARIA

IT 5125 CATANIA

IT $\triangle L Y$

PAULS THOMAS ALBERT DR

NPI FUER RADIOASTRON

AUF OEM HUEGEL 69

$D=53 \mathrm{O}$ BONN 1

PAXTON HAROLDJ B R

RCYAL GQEENW ICH OBS

HEOSTNCNC

HAILSHAN

E SLSSEX AN27 IRP

U.K.
PAOLICCHI PAOLO DR

OSSER VATORIO ASTRO-

NOM ICO

MERATE

MILANO

I TALY

PAPALOIZOU JOHN C B DR

INST OF ASTRONOMY

MADIAGLEY ROAD

CAMBR IDGE CB 3 OHA

U.K.

PARCELIER PIERRE DR

OBSERVATOIRE

DE PAR IS

61 AV L OBSERVATOIRE

F-75014 PARIS

FRANCE

PARIJSK IJ YU N DR

SPEC IAL ASTROPHYS-

LENINGRAD RRANCH

196140 LENINGRAD

U.S.S.R.

PARKER ROBERT A R

COIDE CB

NASA-JSC

HOUSTON TX 77058

$U \cdot S \cdot A$.

PARKINSON WILLIAM H

HARVARD COLLEGE

DRSERVATORY

60 GARDEN ST

CAMRRIDGE MA 02138

U.S.A.

PARSONS SIDNEY B DR

UEPT OF ASTRQNOMY

AUSTIN TX 78712

U.S.A.

PASACHOFF JAY M PROF

WILLIAMS COLLLEGE

HOPK INS OESERVATORY

WILLIAMSTOWN MAO1267

$U . S . A$.

PASINETTI LAURA E PROF

OSSER VATOR IO DI

MILANG MERATE

$\checkmark I A$ B IANCHI 46

$I=22055$ MERATE

PATHR IA RAJ K PROF

DEPT OF PHYSICS

WNIVERSITY WATERLOQ

CANADA

PAVLOV NIKOLAJ N PROF DR PULKOVO ORSERVATORY 196140 LENINGRAD

U.S.S.R.

PEACH G DR

DEPT PHYSICS ASTRO

UN IVER S I TY COLLEGE

GOWER STREET

LONDON WCIE $68 T$
PAPAGIANNIS MICHAEL D PRO

DEPT OF AS TRONOMY

BOSTON UNI VERS ITY BOSTON MA OLZIS

U.S.A.

PAPATHANASOGLOU D DR

DEPT OF ASTRONOMY

UNIV OF ATHENS

PANEPISTIMI DPOL IS

A THENS 621

GREECE

PARESCE FRANCESCO DR

SPACE SCIENCES

LABORATORY

UNIVERSITY OF CALIF

BERKEL

PARKER EDWARD A DR

ELECTRONICS

LABORATORIES

THE UNIVERSITY

CANTERBURY CT2 7 NT

U.K.

PARKINSON JOHN H DR

MULLARD SPACE SC LAB

UNIV COLLEGE LONDON

HOL MBURY ST MARY

DORKING SURREYRH56NS

U.K.

PARRISH ALLAN UR.

STATE UNIV. OF N.YORK

$C / 06191$ GRC U MASS.

AMHERST MA OLOO3

U.S.A.

PARTHASARATHY M DR INDI AN INSTITUTE OF

ASTROPHYSICS

BANGALORE 560034

I NDI A

PASCOAL ANTONID J B SCI

OBS ASTR.ONOMIC

PROF MANUEL D BARROS

MONTE DA VIRGEM

VILA NDVA DE GAIA

PORTUGAL

PASTORIZA MIRIANI G DR

INSTITUTO DE FISICA

UNI VERSIDADE FEDERAL

DO RI O GRANDE DO SUL

GRAZIL

PAULINY TOTH IVAN K K DR

MPI FUER RADIOASTRO-

NCMIE

AUF DEM HUEGEL 69

D-53O 3 BONN

PAVLOVSKAYA E D DR

STERNBERG STATE AS-

TRONOMICAL INSTITUTE

117234 MOSCOW

U.S.S.R.

PEACH JOHN $V$ DR

DEPT OF ASTROPHYSICS

SOUTH PARKS ROAD

OXFURD OXI $3 R Q$

U.K. 
PEALE STANTON J FROF REPT UF PHYS ICS

CELIFOKVIA

SA EAREARA CA 93106

U.S.A.

PECK[R J C PR[JF

$3 \mathrm{G}$ P.IS RLVD ARACO

F-15 14 PARIS

PECERSEV CLAF PROF

UISTCRY SCI ENCE DEPT

MY NENKEGADE

(K-B.S) AARHUS $C$

DENNARK

PEEBLES P JAMES E

JCSEPH HENRY

PRINCETON NJ O854:

U.S. A.

PEKERIS CHAIM LEIP PROF

CEPT APPLIED MATHS

WEIL INN INST ITUTE

REHCVOT

REHCVOT

PELSEVEER JEAN KON PROF

UNIV LIERE DE RRUX

76 AV DES TRENADIERS

P.C. 6

B-II O BRUSSELS

PEANY C J A MISS
RCYAL GREENWICH
CESERATORY
HAISHAM
EAST SUSSEX 3N27 IRP
U.K.

PENZIAS ARNO A CR

EELL LABQRATORIES

ECX 4 :

U.S.A.

PERDAIU JEAN M CR

CASTKEPHYSI QUE

E-420) COINTE-DUGREE

FELGIUN

PEREZ-PERAZA J CR

INST DE ASTRONOMIA

INAM POSTAL 7D-264

NEXICO CF

NEXICD

PERCLA G C.DR

ISTITUTO FैIS ICA

IIA CELCRIA I6

$1=291$

PERRY JUDITH J DR IPI FUER PHYSIK UND

ASTRCPHYSIK

FEEHRINGEK RING 6

C-Q.J1O MUENCHEN 4\%

GEHNAVY, F.R.
PEARSE REGINALD W 8 DR GO GREENLANDS ROAD

STAINES MDX TWI8 4LR

U.K.

PEDER SEN $B$ DR

OBS DE MEUDON

FRANCE

PEDLAR ALAN DR

NRAL

JODRELL BANK

CFESHIRE SKII 9DL

U.K.

PEERY BENJAMIN F PROF DEPT OF PHYSICS AND

HOWARO UN I VER SI TY

WASHINGTON DC 20059

U.S.A.

PEL J W DR

KAPTE I JN STERREWACHT

RODEN 8120

THE NETHERLANDS

PELTIER LESLIE C

DELPHOS OH 45833

U.S.A.

PENSADO J DR

OBSERVATORIO

ASTRONOMICO

ALFONSO XII 5

MADR ID 7

SPAIN

PERAIAH ANNAMANENI OR

ASTROPHYSIC

ANGALORE 560034

INOIA

PEREK LUEOS DR

UNIV OF CALIFORNII

SAN DIEGO PO BOX 109

LA JOLLA CA $92037^{10}$

U.S.A.

PER INOTTO MARIO DR

FISICO ARCETRI I-5OI 25 FIRENZE

ITALY

PERR IN MAR IE-NOELLE DR OBSER VATOIRE

DE PAR IS

6 I AVE OBSERVATOIRE F-75014 PARIS

PERSIDES SOTIRIOS C ASST PROF

UNIV OF THESSALONIKI

THES SALON IK I
PEAT $D W D R$

THE DBSERVATORIES

MADINGLEY ROAD

CAMBRIDGE CBS OHA

U.K.

PEDERSEN HOLGER DR

ESO 0

CASI LLA 16317

CHILE

PEDOUSSAUT ANDRE

I AVE FLAMMARIDN

F-3I500 TOULDUSE

PEIMBERT MANUEL OR INST DE ASTRONOMIA APDO POSTAL 70264 MEXICO 20 DF MEXICO

PELLAS PAUL DR

G1 RUE BUFF ON

FRANCE

PENNY ALAN JOHN DR S A ASTRON OBS ORSERVATORYY 7935 CAPE SOUTH AFRICA

PENSTQN MICHAEL $V$ DR VI LLAFRANCA SATELLIT TRACKING STATION PO BOX APT 54065 MADR I D

SPAIN

PERCY JOHN R PROF ERINDALE COLLEGE MISSI SAUGA ONT L5L IC6

PEREZ-DE-TEJADA H A DR INST DE ASTRONOMIA UNAM

AV GASTELUM ENSENADA BAJA CALIFORNIA
MEXICO

PERKINS FRANCIS $W$ DR PLASMA PHYSICS

LABORATORY

$P$ O BOX 451

PRINCETON NJ 08540

U.S.A.

PERRY CHARLES L DR

DEPT OF PHYSIASTRON

LOUISI ANA STATE UNIV U.S.A.

PESCH PETER DR WARNER AND SWASEY ORSERVATORY

1975 TAYLOR RD

E ČLEVELAND OH 44112 
PETERS GERALDINE JOAN DR UNIV SOUTHERN CAL IF LCS ANGELES CA 90024 U.S.A.

PETERSQN CHARLES JOHN CR 1) $E$ PT OF PHYS ICS 223 PHYSICS RLDG CCLUMBIA MO 65211 U.S.A.

PLTFLRD A DAVID OR DEPT OF ASTROPHYSICS SCUTH PARKS ROAC CXFORD OXI $3 R Q$ U.K.

PETREPOULOS BASIL CH CR RES CTR ASTRO AP MAT ACACEMY OF ATHENS ATHENS 136 GREECE

PETRCV G M DR AIKOLAEV DEPT OF THE NAIN ASIRONOMICAL OPSERVATORY $32700 \%$ NIKOL AEV U.S.S.R.

FETIENGILL GORDCN H PROF PN 54-620

NASS INSTITUTE OF

TECHNCLOGY

CAMBRIDGE MA 02139

U.S.A.

PEYTURAUX ROGER H PROF

INST O ASTROPHYSIGUE

$98 \mathrm{Q}$ BLVD ARAGO

F-75OI 4 PARIS

FRANCE

PFLUG KLAUS DR

ZENTRALINSTITUT FUER

CER- 15 POT SDAM-

TELEGRAFENBERG

G.D.R.

PHILLIPS JOHN G PROF

UAIV OF CAL IFORN IA

EOI CAMPBELL HALL

EERKELFY CA 94720

U.S.A.

PICK MONIQUE DR

CASCP PSATOIRE

DE NEUDCN

$F-92190$ MEUDON

FRANC :

PIERCE DAVID ALLEN

PHYS ICAL SCS DIV

ELCAMINO COLLEGE

U.S.A.

PILCHER CARL BERNARD OR

INST FOR ASTRCNOMY

UNIV UF HAWAII

2600 WCODLAWN DRIVE

HCNOLULU HI 96822

U.S.A.
PETERS WILLIAM L III DR ASTRONOMY DEPT

UNIV OF TEXAS

U.S.A.

PETER SON LAURENCE E PROF

DEPT OF PHYSICS COII

AT SAN DIEGO

AT JAL DIEGO 92093

U.S.A.

PETR I WINFRIED PROF DR

UNTERLEITEN 2

POSTFACH 106

8162 SCHL IERSEE OBB

GERMANY, F.R.

PETROSIAN VAHE PROF

INSTITUTE FOR PLASMA

RESEARCH

STANFORD UNIVERSITY

STANFORD CA $943 \mathrm{C5}$

U.S.A.

PETROV NIKOLAI

ASTRONOMICAL OBS

VARNA

BULGARIA

PETTIT E DR

MT WILSON AND

PI3 ST BARBARA ST

PASASENA CA 91106

U.S.A.

PFENNIG HANS H DR

MPI.F PHYSIK UND

ASTROPHYSIK

FOEHR INGER RING 6

GERMANY UENCHEN

PHAM-VAN J MME

CERGA

ÁVENUE COPERNIC

F-06130 GRASSE

FRANCE

PHILLIPS THOMAS GOULD DR BELL TELEPHONE LABS

MURRAY HILL NJ 07476

$U . S \cdot A$.

PIDDINGTON JACK H RES FEL NATIONAL MEA SUREMENT

LABORATORY P O B 218

LINDFIELD

SYDNEY 2070

AUSTRALIA

PIIROLA VILPPU E DR

TAHT I TCRN I NMAK I

SF-OO 130 HELSINKI 13

FINLAND

PILKINGTON JOHN D H DR ROYAL GREENWICH

DESER VATORY

HAIL SHAM

EAST SUSSEX BN27 IRP

U.K.
PETERSON BRUCE A DR

ANGLO AUSTRALIAN

OBSERVATORY

$P$ B BOX 296

EPPING NSW 2121

AUSTRALIA

PETERSON RUTH CAROL DR

607 MARI ON PLACE

PALD ALTO CA 94301

U.S.A.

PETRINI DANIEL DR OBSERVATOIRE DE NICE

B-P 252
FRANCE

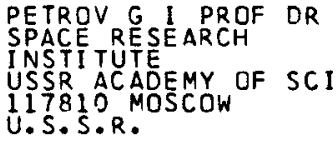

PETROVSKAYA M S DR INSTITUTE OF THEORETICAL ASTRONOMY

192187 LENINGRAD

U.S.S.R.

PEYTREMANN ERIC DR 8 SA
F- 757538 RUE MARIO NIKIS FRANCE

PFLEIDERER JORG PROF INST F ASTRONOMIE UNI VERSITAETSSTR 4

A-6O2O INNSBRUCK

PHILIP A G DAVIS

DUDLEY OBSERVATORY

1202 TROY-

SCHENECTADY RD

LATHAM NY 12110

U.S.A.

PICAT J P DR

F-92190 MEUDON

FRANCE

PIERCE A KEITH DR

OBSERVATORY

PO 26732

TUCSONAZ 85726

U.S.A.

PILACHOWSKI CATHERINE DR A STRONDMY DEPT FM-20 UNIV OF WASHINGTON SEATTLE WA 98195

U.S.A.

PILOWSKI K PROF DR GEODAETISCHES INST TECHN UNI VERS I TAET NIENBURGER STR 1 D-3ODO HANNDVER GERMANY, F.R. 


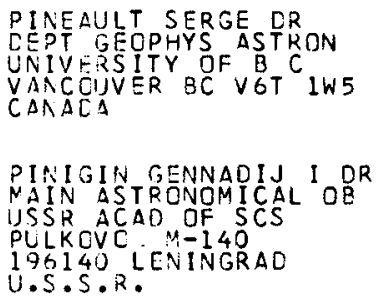

PCLETTO GIANNINA PRDF OSSERVATORIO ARCETRI LARGC FERMI 5

I -50125 FIRENZE

ITALY

PCLCZHENTSEY OIMITRIJ COR PULKCVE OBSERVATORY

$19614 \%$ LENINGRAD

U.S.S.R.

PCNSONAY JOHN E A DR

NRAL

JCDRELL BANK

CHESHIRE SKII 9CL

U.K.

PCPELAR J DR

GRAVITY AND GEOC CIV EARTH PHYS ICS BRANCH 3 CBSERVATORY CRESC

OTTAWA CNT KIA OE 4

CANALA
PINES DAVID PROF

UNIVER SITY ILLINOIS

URBANA IL GI 801

U.S.A.

PINKAU K PROF

MPI FUER EXTRATERRE

STR ISCHE PHYSIK

D- 8046 GARCHING

GERMANY, F.R.

PIPHER JUDITH L

MY DEP I

UNIV OF ROCHESTER

ROCHESTER NY 14627

U.S.A.

P ITZ ECKHART DR

MP I-ASTRONOMY

D-69CO HE IDELBERG

GERMANY, F.R.

PLASKETT HARRY HEMLEY

48 8LENHE IM DR IVE

OXFORD OX 2800

U.K.

PLAVCOVA Z DR

DEPT OF ASTRONOMY

UCLA

405 HILGARD AVE

LOS ANGELES CA 90024

-S.A.

PODOBED V V DR

STERNBERG STATE AS-

TRONOM ICAL INSTITUTE

117234 MOSCOW

U.S.S.R.

POHL ECKHARD DR

STERNWARTE NUERNBERG

LUETZOWSTR IO

D-850O NUERNBERG

GERMANY, F.R .

POLLACK JAMES B DR

SPACE SC DIV MP245-3

NASA AMES RES CTR

MOFFETT FIELD

CA 94035

U.S.A.

POLUPAN P N OR

KIEV STATE UNI VERSI TY ASTRONOMICAL OBSERVATORY $252053 \mathrm{KIEV}$

U.S.S.R.

POOLEY GUY DR

CAVENDISH LABORATORY MADINGLEY RD U.K.

POPOY N A DR GR AVIMETR ICAL OBSERVATORY OF UKRA INIAN ACADEMY OF SCIENCES 314029 PDLTAVA U.S.S.R.
PINGREE DAVID PROF BROWN UNIV PROVIDENCE RI 02912 U.S.A.

PINTO GIROLAMO PROF

OSSERVATORIO

ASTRONOMICO

I 351 IOO PADOVA

PISMIS DE RECILLAS PARIS

PROF

INST DE ASTRONOMIA

APDO POSTAL 70264

MEXICO

\section{PIZZELLA G DR \\ I ST DI FI I I CA \\ G MARCONI \\ UNI VEI \\ I TALY}

PLASSARD J DR

KSARA DBSERVATORY

KSARA

LEBANON

PLAVEC MIROSLAV PROF

DEPT OF ASTRONOMY

UNI VERSI TY OF

CALI FORNIA MS 8979

LOS ANGELES CA 90024

U.S. A

POEPPEL WOLFGANG G L DR

INSTITUTO ARGENTIND

DE RADI OASTRONOMIA

CASILLA DE CORREO 5

I 894 VILLA ELISA BA ARGENTINA

POLAND ARTHUR I DR

HIGH ALTI TUDE

OBSSERVATORY Y

P D BOX 3000

BOULDER CO 80307

U.S.A.

POLNITZKY GERHARD DR

UNIV WIEN

TUERKENSCHANZSTR 17

$A-1180$ WIEN

AUSTRIA

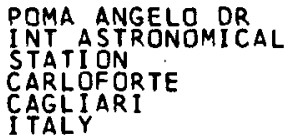

POPE JOHN D

ROYAL GREENWICH

OBSERVATORY

HAILSHAM

EAST SUSSEX BN27 IRP

U.K.

POPQV VICTOR S DR

MAIN ASTRONOMICAL OB

USSR ACAD OF SCS

PULKOVO M-140

196140 LENINGRAD

U.S.S.R. 
PCPGVA MALINA D CR DEPT OF ASTRONOMY QULGARIAN ACAD SCI 7 NCVENBER 1

SCFIA 1200

BULGARIA

PCPPER DANIEL M PROF

DEPT OF ASTRONOMY

UNIVERS ITY OF

CALIF ANGELES CA 90224

PCRTER VEIL A PROF

PHYSICS DEPT

RELF IELC

IRELAND

PCTTER HEINO I CR

PULKCVC OESERVATORY

196140 LENINGRAD

U.S.S.R.

PQUNOS KENNETH A PROF

DEPT OF PHYS ICS

UNIVERSITY

LFICESTER LEI 7RH

U.K.

PRACERIE F DR
CESPA
CPSERVATOIRE MEUCON
F-OS 190 MEUDON
FRAACE

PKEITE-MARTINEZ ANDREA DR LABCRATCRIO OI

ASTRCF
CP 67

I-0OS 44 FRASCAT 1

IT $A L Y$

PRESS WILLIAM H CR

HARV ARD COLLEGE UES

CANBRICGE MA 02138

U.S.A.

PREVCT BURNICHON MARIE

LCIJISE DR

INST ASTROPHYSIQUE

98 BIS BLVD ARAGO

$F-7514$ PAR IS

FRANCE

PFICE I MARCUS CR

DIV ASTRONOMICAL SCS

NAT SCIENCE FOUND

$1870 \%$ STRET N $h$

WASHINGTON DC 20550

U.S.A.

PRINCE HELEN DODSON PROF

4800 FILLMORE AVE

ALEXAVDYIA VA 22311

U.S.A.

PRCBSTEIN R F DR

ENGINEERING

CANBRICGE MA O2139

U.S.A.
POPOVIC BOZIDAR PROF DR FACUL TY OF TRAFFIC

TAKOV SKA 34

IIOOO BEOGRAD

YUGOSLAVIA

PORFIR EV $\vee V D R$

KRUPSKAJA PEDAGOGIC

INST ITUTE

107846 MOSCOW

$U . S . S . R$.

PORUBCAN VLADIMIR DR ASTRONOMICAL INSTI-

TUTE SAV

DUBRAVSKA CESTA A 11

BG930 BRATISLAVA

CZECHOSLOVAKIA

PCULAKOS CONSTANTINE DR RESEARCH CTR ASTROND ACADEMY OF A THENS ATHENS 136

GREECE

PQUQUET A DR

B

F-060D7 NICE CEDEX

FRANCE

PRADHAN DR

NIZAM IAH OBS

HYDERABAD

IND IA

PRENDERGAST KEVIN H PROF DEPT OF ASTRONOMY COLUMBIA UNIVERSITY $538 W 1205 T$

NEW YORK NY 10027

U.S.A.

PRESTON GEORGE W DR

HALE ORSER VATOR IES

813 SANTA BARBARA ST

PASADENA CA 91101

U.S.A.

PREVOT LOUIS OR

OBSERVATOIRE DE MAR SE ILLE

2 PLACE LE VERRIER F-13004 MARSEILLE

FRANCE

PR IEST E R DR

APPLIED MATHS DEPT

THE UN IVER SI TY

KY 16 ISS

U.K.

PRINGLE JAMES E DR

INST OF ASTRONOMY

MADINGLEY ROAD

CAMBRIDGE CE. 3 OHA

U.K.

PROCHAZKA FRANZ $V$ DR UNIVER S I TAETS-

STERNWAR TE

TUERKENSCHANZSTR 17

$A-1180$ WIEN

AUSTR IA
PQPOVIC GEORG IJE DR A STRONOMICAL OBS 11050 BEOGRAD YUGOSLAVIA

PORTER JOHN G DR

WHI TESTONES

HEMPS TEAD LANE

HAILSHAM

SUSSEX BN27 $3 A A$

U.K.

POTTASCH STUART R PROF KAPTEYN LABORATORIUM

POSTBUS 800

GRONINGEN

THE NETHERLANOS

POUMEYRQL FERNAND MR OBSERVATOIRE

DE RORDEAUX

F-33270 FLOUIRAC

FRANCE

POVEDA ARCADIO DR

INST DE ASTRONOMIA

APDD POSTAL 70-264

MEXICO 20 DF

MEXICD

PRATAP R DR

PHYSICAL RESEARCH

LABORATORY

NAVRANG PURA

AHMEDABAO 380009

I NDI A

PRENTICE ANDREW J R DR

DEPT OF MATHEMAT ICS

MONASH UNIVERSITY

CLAYTON VICT 3168

AUUSTRALIA

PREUSS EUGEN DR

MPI FUER RADI O-

ASTRONOMIE

AUF DEM HUEGEL 69

D-5300 BONN

GERMANY, F.R.

PRICE MICHAEL J DR.

5055 E BROADWAY

SUITE $A-214$

TUC SON AZ 85711

U.S.A.

PRIESTER WOLFGANG PROF

INST F ASTROPHYSIK

AUF DEM HUEGEL 71

D-53 OD BONN

PRI TCHET CHRISTOPHER J DR

DEPT GEOPHYS ASTRON

UNI VERSITY OF $B C$

VANC OUVER BC V6T IW5

CANADA

PRODAN Y I DR

STERNBERG STATE AS-

TRONOMICAL INSTI TUTE

117234 MÓSCOW

U.S.S.R. 
PRCIEY PAUL E DR CESERVATOIRE CE LYON

LAVAL
FRANCE

PRCKCF'EVA IRINA A DR PULKCVC OPSERVATORY 13614 LENINGRAD U.S.S.k.

\section{PRCAIK V I DR \\ CRIMEAN ASTROPHY OBS \\ $\triangle C A D$ SCIEISCES USSR \\ CRINEA \\ U.S.S.R. \\ PFCVFRBIO EDOARCO PROF \\ IST CI ASTRONDM I A \\ $\checkmark$ IA CSPEDALE 72 \\ $1=09150$ CAGL IAR I \\ ITALY}

PRZYBYLSKI ANTONI LR

MCUAT STROMLO

CESERVATORY

WCDEN A C T 2606

AUSTRALIA

\section{PUGET JEAN-LOUP DR \\ $\checkmark$ ASTRCPHYSIQUE \\ 98 IIS ELVD ARACO \\ F-75, 4 PARIS}

PURTCN CHRISTOPHER R DR

CRESS OEPT OF PHYS

YCRK. UNIVERSITY

$470 \%$ KEELE STR

CCWNSVIEW ONT MBJIP3

CAA.ACA

PYPER D M DR

ASTRENGMY

TEL-AVIV UNIVERS ITY

TEL-AV IV

ISKAEL

GUERCI FRANCOIS R CR

EQUIPE ETOILES

FRCIOES

CBS CE MEUDON

F-32190 MEUDON

FPANCE

QUINTANA HERNAN DR

NAT RADIO ASTRO OBS

ECGENONT RD

CHARLOTTESVILLE

$\checkmark A 2.901$

U.S.A.

CVIST BERTIL PROF

MATHEMATICS

SF-2050O ABO 50

FINLAND

RACINE RENE DR

DEPT OE PHYS IQUE

UAIVERS ITE DE

NCNTREAL PQ H3C $3 J 7$

CANACA
PROKAKIS THEODORE J DR OBSERVATOIRE

D ATHENES

ATHENS 306

PROKOF'EVA VALENTINA $V$ DR

CRIMEAN ASTROPHYS OB

NAUCHNYJ

334413 CR IMEA

U.S.S.R.

PROTHEROE WILLIAM M PROF

DEPT DOF ÁSTROONOMYY

OHIO STATE UNIV

I74. W $18 T H$ AVE

COLUMBUS OH 43210

U.S.A.

PROVOST J DR

OES DE NICE

$B \quad 252$

FRANCE

PSKOVSKIJ JU P DR

STERNBERG STATE AS-

117234 MOSCOW

U.S.S.R.

PUNETHA LALIT MOHAN DR

UP STATE OBSERVATORY

MANÖRA PEAK

NAIN I TAL 263129

INDIA

PUSHK IN SERGEY B DR

TIME AN FREQUENCY

SERV ICE

GOSSTANDARD USSR

117049 MOSCOW

U.S.S.R.

QUAST G R

OBS NACIONAL

R GENERAL BRUCE 586

SAO CR I STOVAO

2 COOORIO DE JANEIRO BRAZ IL

QUERCI MONIQUE DR

EQUIPE

OBS DE MEUDON

F-92190 MEUDON

FRANCE

QUINTANA JOSE M DR

INSTITUTO DE ASTROF I

SICA DE ANDALUCIA

GRANADA

SPAIN

RAADU MICHAEL A DR

REPT PLASMA PHYSICS

TECHNOLOGY

S-10044 STOCKHOLM 70 SWEDEN

RACKHAM THOMAS W DR

JODRELL BANK

CHESHIRE SK 11 9PL

$\mathrm{U} . \mathrm{K}$.
PROKOFIEV VLADIMIR K PRDF

CRIMEAN ASTROPHYSI-

CAL OBSERVATORY

PO NAUCHNY

334413 CR I MEA

U.S.S.R.

PRONIK I I DR

CRIMEAN ASTROPHY OBS

ACAD SC! IENCES USSR

CRIMEA

U.S.S.R.

PROTICH MILORAD B

ASTRONOMICAL OBSERVA

TORY OF BELGRADE

VOLGINA 7

11050 BELGRADE

YUGOSLAVIA

PRYCE MAURICE H L DR

DEPT OF PHYSICS

2075 WESBROOK MAL

VANCOUVER BC VOT IWS

CANADA

PUCILLO MAURO DR

OSSER VATORIO

ASTRONOMICO

IIA TIEPOLO 11 TI

I $T A L I Y Y$

PURGATHOFER ALOIS PROF DR

INST FUER ASTRONOMIE

UNIV WIEN

TUERKENSCHANZSTR 17

A-I 180 WIEN

AUSTRIA

PYE JOHN P DR

8 BEAUMONT WALK

LEICESTER LE4 OPO

U. K.

QUENBY JOHN J DR

BLACKETT LAB

I MPERIAL COLLEGE

PRINCE CONSORT ROAD

LONDON SW7 2 BZ

U.K.

QUI JANO LUIS

INSTI TUTO Y OBSERVA-

TORI D DE MARINA

SAN FERNANDO CADIZ

SPAIN

QUIRK WILLIAM J OR

HAYWARD CA 9454

UAYWARD.

RACHKOVSKY D N DR CRI MEAN ASTROPHYSIACAD OF SCI NAUCHNIY 334413 CRIMEA

U.S.S.R.

RADHAKRISHNAN $V$ PROF

DIRECTOR

RAMAN RESEARCH

I NST I TUTE

BANGALORE 560006

INDI A 
PADLCVA L N DR INST ITITE OF SCIENCE $T I C N$

$125213 \mathrm{MOSCOW}$

U.S.S.R.

RAGHAVAN NIRUPAMA DR TE CT TECHNCLOGY ITUCANPUS

NEW DELHI 110029

incia

RAINCIND ERNST OR

NETHERL FCUNDATION

FCR A IOIUASTRCNCMY

7991 AC DW INGELCO

THE NETHERL ANCS

PAJCHL JAROSLAV TR

ASTRL YONICAL INST

CZECHOS ACAD

251 t5 CNOREJOV

CZ ECHOSLLV AK I A

RAKCS KARL D PRCJF

IAST FUER ASTRONCMIE

URIV WIEN

TURKENSCHANZSTR 17

$A-1180$ W IEN

AUSTKIA

RAN LTY REUVEN CR

LAE HIGH ENERGY ASTR

FLIGHT CT P COOE ESO

FLIGHT CT R COOE ESO

U.S.A.

RAN:CIC LEO PROF DR

GURLUL ICEVA 54

ZACRE

YUECSLAVIA

\section{RAC A P DR \\ RAQIC ASTRONOMY CTR \\ $P$ C BOX ND 8 \\ CCTACAMUND 643001}

INCIA

RAPLEY CHRISTOPHER G DR

MULLARD SPACE SC LAB

UNIV COL

LCNOCN

$R A L I N V A O R$

RESEARCH INST ITUTE

GI 3600 GORKIJ

GI) $3601.5 \cdot \mathrm{G}$

REAVES GIBSON PROF

UNIVERSITY OF

SCUTHERN CALIFORNIA

LOS $\triangle N$

QEEER GROTE DR

GEAERAL DELIVERY

PCTHWELL TASM 7411

AUSTRALIA
RAEDLER $\mathrm{K} H$ H DR

ZNTR INST ASTROPHYSIK

ASTROP ORSERVATORIUM

TFLEGRAFENEERG

CER - I5 POTSDAM

$G \cdot O \cdot R$.

RAHE JURGEN PROF

UNIV ERL-NUERNBERG

ASTRONOMICAL INST

STERNWAR TSTR 7

D-B OCO BAMBERS

RAINE DEREK J DR

DEPT OF ASTRONOMY

UN IVERSITY OF

LEICESTER

LEICESTER LEI TRH

RAJU $P K$ DR

RAJU P K DR
INDIAN INSTITUTE OF

ASTROPHYSICS
BANGALDRE 560034

IND IA

RAKSHIT H PROF

BENGAL ENGINIEER ING

COLLEGE

HEWRAH

INDIA

RAMBERG JOERAN M PROF

GENVAEGEN 4

$S-13300$

SALTSJUEBADEN

SWECEN

RANK DAVID M PROF

LICK OBSERVATORY

UN IVER SITY OF CALIF

SANTA CRUZ CA 95064

U.S.A.

RAO RAMACHANDRA $V$ PROF

ISRO SATELLITE

BANGALORE $56 \mathrm{CO} 58$

INCIA

RASMUSEN H Q DR

VAER SLEVGAAREN

FRANK ERUPVE I 40

DK -4400 KALUNDBORG

DENMARK

REAOHEAD ANTHONY C S DR

RADIO ASTRONOMY DEP

CAL IFQRN IA INST

OF TECHNOLOGY

U.S.A.

REAY NEWR ICK K DR

ASTRONOMY GROUP

BLACKETT LABORATORY

INPER IAL COLLEG

U.K.

RECILLAS-CRUZ ELSA DR

INST DE ASTRONOMIA

UNAM

APDO POSTAL 70-264

MEXICO D F

MEXICO
RAF ANELLI PIERO DR OSSERVATORI O ASTRONOMICO

$I=35100$ PADOVA

I TALY

RAIKOVA DQNKA OR

BULGARIAN $\triangle C$ OF SCS 7 TH NOVEMBER ST I 1000 SOF IA

BULGARIA RAJAMOHAN R DR ASTROPHYSICS BANGALORE $560 \quad 034$ I NOI A

RAKAVY GIDEON PROF HEBREW UNI VERSITY EINSTEIN INST PHYS JERUSALEM

I SRAEL

RAMAOURAI $S$ DR

TATA INST FUND RES

HOMI BHABHA ROAD

BOMRAY 400005

I ND I A

RAMSEY LAWRENCE W DR

DEPT DF ASTRONOMY

525 DAVEY LAB

LNIV OF PENNA

UNIV PK PA 16802

U.S.A.

RANKIN JDANNA M DR

DEPT OF HISTORY

CORNELL UNIVERSITY

MCGRAW HALL

1 THACA NY 14853

U.S.A.

RAPAPORT $M$ DR

ORS DE BORDEAUX

$F-33270$ FLOIRAC

FRANCE.

RAYROLE JEAN R DR

OBSERVATOIRE

DE MEUDON

F-92190 MEUDON

FRANCE

REASENBERG ROBERT D DR

MIT

54-612

CAMBRIDGE MA 02139

U.S.A.

REBEIROT EOITH DR

OBS DE MAR SEILLE 2 PLACE LE VERRIER FRANCE

REDDI SH VINCENT C PROF ROYAL ORSERVATORY EOINBURGH EHI2 9AJ U. K. 


\section{REES MARTIN J PROF INST OF ASTRONOMY NADINGLEY RD CANPRICGE CA3 OHE $U . K$. \\ RETSEAL S PROF DR HAMRURGER STERNWARTE GCCJEJEERGSWEG 112 D-2D5O HAMBURG 80 GERMAUY, F.R. \\ REID MARK JONATHAN DR NAT RACIO ASTRON OBS ECGENONT RC CHARLOTTESVL VA22901 U.S.A. \\ REINISCH C CR \\ P P 252 \\ FRANCE}

RENSE WILLIAM A ER DEPT PHYS AND ASTROP

UAIV OF COLORADC

CUANE PHYSICAL LABS

FCULDER CO 8030

U.S.A.

REGUIFNE YVES DR

CESERVATOIRE

OE ELRCEAUX

F-33270 FLOIRAC

FRAACE

KEYNCLDS JOHN H PROF DEPT OF PHYS ICS UAIVERSITY OF CALIF PERKELEY CA 94720 U.S.A.

RIBES ELIZABETH CR

CES ERVATOIRE

CE NEUECN

$F=9213 !$ MEUDUN

FRANCE

RICE JCHN D DR

DEPT PHYSICS AND

ASTRCNCMY

BRANCON UNIVERSITY

CRANEOH MAN RTA GAG

CANATA

RICHER HARVEY R OR DEPT GECFHYS-ASTRON UNIVERSITY OF EC 2075 WESBROOK PLACE VANCCUVER BC VGT IWS CANACA

RICHTER N B PROF

PEST ULLA

CLLR -5321 WEIMAR

C.D.R.

KICKER GEURGE $R$ DR

CTR FOR SPACE RES

77 NASS AV RM 37-527

CANERIDGE MA J2139

U.S.A.
REEVES EDMOND M DR

P. 0 BOX 3000

BOULDER CO 80307

U.S.A.

REGO FERNANDES M. DR.

ASTROFISICA

FACULTA FISICA

UNIV COMPLUTENSE

MADR ID 3

SPAIN

REIMERS DIETER OR

INST THEO PHY STERNW OLSHAUSENST GB N $61 \mathrm{C}$ D-2300 KIEL 1

REIZ ANDERS PROF

UNIVER I ITY

OPS SER VATORY

OSTER VOLDGADE 3

OK - 1350 COPENHAGEN K DENMARK

RENSON $P$ F $M$ DR

INSTITUT

D ASTROPHYSIQUE

B-4200 COINTE-DUGREE

BELGIUM

REUN ING ERNEST G DR

ASTRONOMY

UNIV OF GEORGIA

ATHENS GA 30602

U.S.A.

REYNOLDS RONALD J OR

SPACE PHYSICS GROUP

MAEISON WI 53706

U.S.A.

RIBES JEAN-CLAUDE DR INAG

77 AVE DENFERT-

ROCHEREAU

F-75014 PARIS

FRANCE

RICHARDSON E HARVEY DR

DOMINION ASTROPHYSI -

CAL OBSERVATORY

5071 W SAANICH RD

VICTOR IA $B C$ V $8 \times 3 \times 3$

CANADA

R ICHTER G A DR

ZENTRALINSTITUT FUER A STROPHY S IK STERNWARTE SONNEBERG $G . D . R$.

RICKARD JAMES JOSEPH DR CLARK LAKE RAOID OBS

P O BOX 626

BORREGO SPRINGS

CO 92034

U.S.A.

RICKETT RARNABY JAMES DR DEPT $\triangle P P L I C D$ PHYSICS

AND INFORMATION SC

UN IV CALIF SAN DIEGO

LA JOLLA CA 92093

U.S.A.
REEVES HUBERT PROF

CEN SACLAY

B-PILOO GIF S YVETTE
FRANCE

REID JOHN H DR IOO7 RESEDA DRIVE HOUSTON TX 77062 U.S.A.

REINHARDT MICHAEL PROF

A STRONOMI SCHES INST

RUHR UNI VERS I TAET

POSTFACH 102148

$D-4630$ BOCHUM

GERMANY, F.R.

REMY BATTIAU LILIANE G A

DR

INST D ASTROPHYSI QUE

UNI VERSITY DE LIEGE BELGIUM COINTE DUGREE

RENZINI ALVIO PROF

DSSERVATORIO

VIA $2 A M B O N I$
I -40126 BOLOGNA
ITALY

REVELLE DOUGLAS ORSON DR

DEPT OF PHYSICS

C U BOX 6010

NOR THERN ARIZ UNIV

FLAGSTAFF AZ BOOII

U.S.A.

RHYNSBURGER ROBERT W DR US NAVAL OBSERVATORY WASHINGTON OC 20390

U.S.A.

RICE D A

SURVEY

U.S.A.

RICHARDSON R S

GRIFFITH OBS

LOS FELIX STATION

LOS ANGELES CA 90027

U. S.A.

RICHTER JOHANNES PROF

INST F EXPER PHYSIK

PHYSIKZENTRUM

D-2300 KIEL

GERMANY, F.R.

RICKARD LEE J DR

NAT RADIO ASSTRON OBS

EOGEMONT RD

U.S.A.

RICKMAN HANS DR

ASTRONOMISKA OBS

$80 X 515$
$S-75120$ UPPSALA

SWEDEN 


\begin{tabular}{|c|c|}
\hline $\begin{array}{l}\text { RICELE ANTHONY C CR } \\
\text { UEPT ASTRO-GEOPHYS }\end{array}$ & $\begin{array}{l}\text { R IEGEL KURT W DR } \\
\text { DEPT OF ASTRONOMY }\end{array}$ \\
\hline $\begin{array}{l}\text { CLLCRADO CO } 80302 \\
\text { ECULEER CO } 8030.4\end{array}$ & $\begin{array}{l}\text { LCLA ANGELES CA } 90024 \\
\text { U.S.A. }\end{array}$ \\
\hline $\begin{array}{l}\text { RISHINI ALRERTO PROF } \\
\text { CSS ERVATORIOAASTRO- } \\
\text { FISICO ARCETRI } \\
\text { LARGO A FERMI } 5 \\
\text { ITSCI } 125 \text { FIRENZE } \\
\text { ITALY }\end{array}$ & $\begin{array}{l}\text { RIGHINI-COHEN GIOVANNA DR } \\
\text { DEPT EARTH SPACE SCS } \\
\text { SUNY } \\
\text { STONY BROOK NY } 11794 \\
\text { U.S.A. }\end{array}$ \\
\hline $\begin{array}{l}\text { RIIHIMAA JQRMA J OR } \\
\text { AARNE KARJALAINEN } \\
\text { CPSERVATORY } \\
\text { UNIVERSITY OF OULU } \\
\text { SF-9OIDO OULU IO } \\
\text { FINLAND }\end{array}$ & $\begin{array}{l}\text { RILEY JULIA M DR } \\
\text { MULLARD RADIO ASRON } \\
\text { LABORATORY } \\
\text { MADINGLEY ROAD } \\
\text { CAMBR IDGE } \\
\text { U.K. }\end{array}$ \\
\hline $\begin{array}{l}\text { RINEHART JOHN S PROF } \\
\text { HYPERDYNAMICS } \\
\text { P C } 3 O X \quad 392 \\
\text { SANTA FE NM } 87501 \\
\text { U.S.A. }\end{array}$ & $\begin{array}{l}\text { RING JAMES PROF } \\
\text { THE BLACKETT } \\
\text { LABORATORY } \\
\text { IMPER IAL COLLEGE } \\
\text { LONDON SW7 2BZ } \\
\text { U.K. }\end{array}$ \\
\hline $\begin{array}{l}\text { RINGUELET ADELA E DR } \\
49342 \text { A PLATA } \\
\text { I9OO LA A } \\
\text { ARGENTINA }\end{array}$ & $\begin{array}{l}\text { RITTER HANS DR } \\
\text { MPI FUER PHYSIK UND } \\
\text { ASTROPHYSIK } \\
\text { FOEHRINGER RING } 6 \\
\text { D-8OOOMUENCHEN } \\
\text { GERMANY, F.R. }\end{array}$ \\
\hline $\begin{array}{l}\text { RCACH FRANKLIN E } \\
2969 \text { KALAKAUA AVE } \\
\text { AFT GOS } 5 \text { HI } 96815 \\
\text { HINCLULU HI } \\
\text { U.S.A. }\end{array}$ & $\begin{array}{l}\text { ROARK TERRY P PROF } \\
\text { OEPT OF ASTRONOMY } \\
\text { OHIO ST UNIVERSITY } \\
174 \text { W I TH AVE } \\
\text { COLUMBUS OH } 43210 \\
\text { U.S.A. }\end{array}$ \\
\hline $\begin{array}{l}\text { KCEE H A G DR } \\
\text { IAST ITUT } \\
\text { DSSTROPHYSIQUE } \\
\text { B-42OO COINTE-OUGREE } \\
\text { EELGIUN }\end{array}$ & $\begin{array}{l}\text { ROBERTI GIUSEPPE DR } \\
\text { OSSERVATORIO ASTRO- } \\
\text { NOMICO } \\
\text { NAPOLI } \\
\text { ITALY }\end{array}$ \\
\hline $\begin{array}{l}\text { RCPERTS DAVID HALL DR } \\
\text { RES LAB ELECTRONICS } \\
\text { 2E-32I } \\
\text { MIT } \\
\text { UAMHRIDGE MA } 02139 \\
\text { U.S.A. }\end{array}$ & $\begin{array}{l}\text { RORERTS JAMES A DR } \\
\text { CSIRO DIVISION OF } \\
\text { RADIOPHY ICS } \\
\text { PODOX 76 } \\
\text { EPPING NSW } 2121 \\
\text { AUSTRALIA }\end{array}$ \\
\hline $\begin{array}{l}\text { RCQERTS WALTER ORR DR } \\
\text { ASPEN INST HUM STUE } \\
\text { I 229 UNIVERSITY AVE } \\
\text { LND FLCOR } \\
\text { BCULDER CO } 80302 \\
\text { U.S.A. }\end{array}$ & $\begin{array}{l}\text { ROBERTS WILLIAM W JR PROF } \\
\text { OPT APPL MATH COM SC } \\
\text { THORNTON HALL UNIVVA } \\
\text { CHARLOTESVILLE } \\
\text { VA 229OI } \\
\text { U.S.A. }\end{array}$ \\
\hline $\begin{array}{l}\text { RCEINSCN BRIAN J DR } \\
\text { CSIRO RADIOPHYSICS } \\
\text { SAECRATORY } \\
\text { EC FOX 76 } \\
\text { EPPING NSW } 2121 \\
\text { AUSTRALIA }\end{array}$ & $\begin{array}{l}\text { RUB INSON EDWARD LEWIS DR } \\
\text { DEPT OF ASTRONOMY } \\
\text { UNIV OF TEXAS } \\
\text { AUSTIN TX } 78712 \\
\text { U.S.A. }\end{array}$ \\
\hline $\begin{array}{l}\text { RCEINSCN LLOYD B OR } \\
\text { LICK OESERVATCRY } \\
\text { UAIVERSITY OF CAL IF } \\
\text { SANTA CRUL CA } 95064 \\
\text { U.S.A. }\end{array}$ & $\begin{array}{l}\text { ROBINSON WILLIAM J DR } \\
\text { DEPT OF MATHEMATICS } \\
\text { THE UNIVERSITY } \\
\text { BRADFORD BDT IDP } \\
\text { U.K. }\end{array}$ \\
\hline $\begin{array}{l}\text { RCESCN IAN E CR } \\
\text { OIV PHYS AND ASTRON } \\
\text { PRESTON POLYTECHNIC } \\
\text { PRESTCN PRI 2TQ } \\
\text { U.K. }\end{array}$ & $\begin{array}{l}\text { ROCCA-VQLMERANGE B DR } \\
\text { LAB RENE BERNAS } \\
\text { BAT } 1086 \text { ORSAY } \\
\text { F- } 1406 \text { ORSAY } \\
\text { FRANCE }\end{array}$ \\
\hline
\end{tabular}

RIEU N G DR DRSERVATOIRE

$F=92190$ MEUDON

FRANCE

RIGUTTI MARIO PROF OSSERVATOR IO ASTRONO MOI ARI ELLO 16 ITALY

RINDLER WOLFGANG PROF UNI VERSITY OF TEXAS RICHARDSON TX 75080 U.S.A.

RINGNES TRULS S DR INST THEOR ASTROPHYS UNIVERSITY OF OSLO $P$ O BOX 1029 BLINDERN OSLO 3 NORWAY.

RIZVANOV NAUFAL G DR ENGELHARDT OBS 422526 KASAN

U.S.S.R.

ROBBINS R ROBERT PROF UNIVERS ITY OF TEXAS

ASTRONOMY DEPT

AUSTIN TX 78712

U.S.A.

ROBERTS BERNARD DR

DEPT APPLIED MATH

UNIV OF ST ANDREWS

ST ANDREWS FIFE

KY16 8QP

U.K.

ROBERTS MORTON S DR

NAT RADIO ASTRO OBS

EDGEMONT RD

CHARLOTTESVILLE

VA 2290

U.S.A.

ROBERTSON WILLIAM H BSC SYONEY OBSERVATORY OBSERVATORY PARK

SYDNEY NSW 2000

AUSTRALIA

ROBINSON I PROF

UNIVERSITY OF TEXAS

BOX 688

MAI OSTATION BE 32

RICHARDSON TX 75080

U.S.A.

ROBLEY R DR OBSERVATOIRE

DU PIC DU MIDI

1 AVE C FLAMMARION F-31500 TOULOUSE FRANCE

ROCHESTER MICHAEL G PROF PHYSICS DEPT ST JOHNS 
RECCIER C DR

CEPT DIASTROPHYS IGUE UAIVERSITE DE NICE

PARC VALRORE

F- 26 U 34 NICE CECEX

FRANCE

RCONAIN RICHARD B CR

¿S LCCUST AVE

LEXINGTON MA O2173

U.S.A.

RCECER ROEERT C PROF

DEPT OF ASTRONONY

SCARPCROUGH COLLEGE

W265 MILITARY TRAIL

CANADA

RCENINAENG RERNT O DR

CASALA SPACE

CUSERVATORY

SWECEN
SW ONSALA

RCGERSCN JOHN B PROF PR INCETON UN IVERSITY DEPT ASTROPHYS SCS

PEYTON HALL 08540

U.S.A.

RCLAND GINETTE DR

INST D ASTROPHYS IQUE

UNIVERSITE DE LIEGE

EET 2 OU

RCNANA ANT ONIO DR

CESEHVATORIO CEL

ERKC

RCCULTAS

TEKTOS $\triangle$ BARCELONA

SPAIN

RCNANCV YURI S DR

CDESSA ASTRONUMICAL

CBSERVATORY

SHEVCHENKO PARK

270014 ODESSA

U.S.S.R.

RCCQ HERBERT J

52 ELIZABETH ST

SCUTH BOUND BROOK

$\mathrm{NJ}$ (.8880

U.S.A.

RCSADO MARGARITA DR

IAST DE ASTRONOMIA

UNAM

APCO POSTAL 70-264

MEXICO D

RCSEN E DR

CEPT OF HISTORY

CITY CCLLEGE OF THE

CITY UNIV NEW YORK

NEW YORK NY 10031

U.S.A.

RESINO LEONIDA PROF

ASTRENCMICAL OBSERVA TCRY PADOVA

PAOCVA
RODOIER FPANCOIS PROF

UNIVER SITE DE NICE

PARC VALROSE

F-06034 NICE CEDEX

FRANCE

RODONO MARCELLO DR

OSSERVATORIO

ASTROFISICO

CITTA UNIVERSI TARIA

IT 9525 CATANIA

RDEMER ELIZABETH PROF

LUNAR AND PLANETARY

LABORATORY

UNIVERSITY ARIZONA

TUCSON AZ 85721

U.S.A.

ROGER ROBERTS S DR

DOMINION RADIO ASTRO

PHYSICAL OBSERVATORY

$B O X 248$

PENTICTON BC V $2 A \quad 6 K 3$

CANADA

ROGSTAO DAVID H DR

MAIL CODE IOZ-24

1201 E CALIF BLVD

PASADENA CA 91125

U.S.A.

ROLLAND ANGEL DR

JUPITER 2

HUETOR VEGA

GRANACA

SPAIN

ROMANCHUK $P R$ DR

KIEV STATE UNI VERSI-

TY ASTRONOMICAL

ORSER VATORY

252053 KIEV

U.S.S.R.

ROMPOLT BOHDAN DR

ASTRONOAICAL INST

UL KOPERNIKA II

51,622 WROCLAW

ROOD ROBERT T DR

BOX 3818 UNIVERSITY STATION

CHARLOTTESVILLE

VA 22903

$\mathrm{U} \cdot \mathrm{S} \cdot \mathrm{A}$.

ROSCH JEAN PROF DIR
OBSERVATOIRES DU PIC

DU MID I ET DE

TOULOUSE

F-65200 BAGNERES

FRANCE

ROSENBERG J DR

SERVAAS BOLWERK 13

UTRECHT

THE NETHERLANDS

ROSLUND CURT DR

DEPT OF ASTRONOMY

CHALMERS UNI VER I TY

OF TECHNOLOGY FACK

$S-40220$ GOETEBORG

SWEDEN
RODGERS ALEX W DR

MT STROMLO

ORSERVATORY

WODEN PO ACT 2606

AUSTRALIA

RODR IGUEZ LUIS F

INST DE ASTRONOMIA

UNAM

APDO POSTAL 70-264

MEXICO D F

ROEMER MAX PROF

INST F ASTROPHYSIK

EXTRATERR FORSCHUNG

AUF DEM HUEGEL 71

AUF DEM HUEG

GERMANY, $F \cdot R$.

ROGERS ALAN E E DR HAYSTACK OBSERVATORY WESTFORD MA D1886 U.S.A.

ROHLFS K PROF DR RUHR UNIV BOCHUM INST FUR ASTRUPHYS

POSTFACH 102148

D-4630 BOCHUM 1

GERMANY, F.R.

ROMAN NANCY G DR

ASTRDPHYS DIV NASA

CODE SC -7

WASHINGTON DC 20546

U.S.A.

ROMANO GIULIANO PROF

VIALE S ANTONIO

I TAI 1 OO TREVISO

RONAN COLIN A

13 ACORN AVE

CAMBRIDGE CB3 BDT

U. K.

ROOSEN ROBERT G DR

LAB FOR SOLAR PHYS

ALBUQUERQUE NM 87131

U.S.A.

ROSE WILLI AM K DR

UNI VERSITY MARYLAND

COLLEGE PK MD 20742

U.S.A.

ROSENDHAL JEFFEREY D DR

NASA HEADQUARTERS

WASHINGTON OC 20546

WASHINGTO

ROSS DENNIS K PROF

IOWA ST UNI VERSITY

AMES IA 50010

U.S.A. 


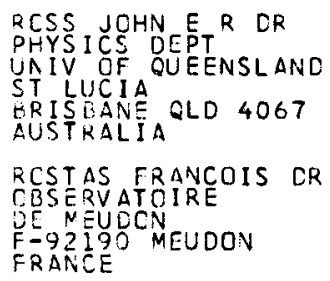

KETS ARNOLD H CR NAT RALIO ASTRCN OBS SCCORRO NM 87801 U.S.A.

RCUNTREE LESH JANET DR LABORATORY ASTRCNOMY SCLAR PHYS CODE GQI GREENEELT MD 20771 U.S.A.

KCUSSEAU JEANINE DR CESERVATOIRE DE LYON FRANCE

RCVITHIS PETER EMM DR WAT ICNAL OBSERVATORY CF ATHENS

ATHE SS 306

CRECCE.

\section{RCWSCN BARRIE DR \\ $N R A L$ \\ JCLRELL RANK \\ CPACLESFIELD \\ U.K. \\ RCY JEAN-RENE \\ DEPT DE PHYSIGUE \\ UNIVERSITE LAVAL \\ CIIE UNIVERS ITAIRE \\ CANALIA}

RUPASHEV HORIS $M$ CR

PULKCVC OBSERVATORY

$13614^{\circ}$ LENINGRAC

U.S.S.R.

RUCIMSKI SLAWOMIR M DR $\triangle S T R C N O M I C A L$

CESERVATORY

AL UJAZDOWSKIE 4

OO 478 WARSZ AWA

PCLAND

KUEK JUHING MOGENS PROF ASTRENOMISK INST ITUT

LANGELANDSGAD

CK-BTO AARHUS C

$D E N M A K K$

RUTENER FREDY $G$ PROF

CESEKVATOIRE

DE GEVEV

CH-129\% SAUVERNY GE

SWITLERLAND
ROSSELAND SVEIN PROF

UN IVER SITY OF OSLO

P O BOX 1029

BLINDERN OSLO 3

NORWAY

ROTH MAR IA-LUISE PH D

HAMBURGER STERNWARTE

D-205 HAMBURG 80

GERMANY, F.R.

ROTTENBERG J A DR

$29 I I$ BAYVIEW AVE

SUITE $110 \mathrm{C}$

WILLOWDALE ONTM2K1E8

CANADA

ROUSE CARL A DR

627 15TH ST

DEL MAR CA 92014

U.S.A.

ROUTLEDGE DAVID DR

ELECTR ICAL ENG

$\triangle L B E R T A$

ELMONTON TGG 2J1

CANADA

ROVITHIS-LIVANIOU H PH D

ASTRONOMY OEPT

UNIV OF ATHENS

PANEPISTIMIOPOLIS

ATHENS 621

GREECE

ROXBURGH IAN W PROF

DEPT APPLIED MATHS

QUEEN MARY COLLEGE

MILE END ROAD

LONDON E 1

U.K.

RCZELOT JEAN $P$

150 BLVD DIDEROT

IMMEUBLE F 128

F-75012 PARIS

RUBEN G PROF DR

ZNTR INST ASTROPHYSIK

ASTROPHYS OBS

TELEGRAFENBERG

DDR - I5 POTSDAM

G.D.R.

RUDDY VINCENT P DR

RECIONAL TECHNICAL

COLLEGE

ROSSA AVE

CORK

IRELAND

RUDN ICK LAWRENCE DR NAT RADIO ASTRON LAB ECGEMONT RO

CHARLOTTESVL VA22901

U.S.A.

RUGGE HUGO R DR

THE AEROSPACE

CORPORATION

P O BOX 92957

LDS ANGELES CA 90009

U.S.A.
ROSSI BRUNO B PROF

MASS INSTITUTE OF

TECHNOLOGY

CAMBRIDGE MA 02139

U.S.A.

ROTH MIGUEL R DR

INST DE ASTRONOMIA

APDO POSTAL 70-264

MEXICO D F

MEXICO

ROUEFF EVELYNE $M$ A OR

QBSERVATOIRE MEUDÔN

DEPT D ASTRDPHYSIQUE

FDTLE 92190 MEUDON

FRANCE

ROUSSEAU JEAN-MICHEL MR

OBSERVATOI RE

DE BORDEAUX

$F-33270$ FLOIRAC

FRANCE

RQUTLY PAUL M DR

US NAVAL DESERVATORY

$34 T H$ AND MASS AVE NW

WASHINGTON DC 20390

U.S.A.

ROWAN-ROBINSON MICHAEL DR

DEPT OF APPLIED MATH

QUEEN MARY COLLEGE

MILE END RD

LONDON E1 $4 \mathrm{NS}$

U. $k$.

ROY ARCHIE E PROF

DEPT OF ASTRONOMY

GLASGOW UNIVERS ITY

GLASGOW GI2 800

U.K.

ROZHKOVSKIJ DIMITRIJ A

PRO

ASTROPHYSICAL

INSTI TUTE

48068 ALMA ATA

U.S.S.R.

RUB IN VERA C DR

DEPT TERRESTRIAL MAG

CARNEGIE INST WASH

5241 BRDAD BR RD N W WASHINGTON DC 20015

U.S.A.

RUDERMAN MALVIN A

PHYSICS DEPT

COLUMBIA UNI VERSITY

NEW YORK NY 10027

U.S.A.

RUDNICKI KONRAD PROF

ASTRONOMICAL OBS

UL ORLA 171

30244 KRAKOW

POLAND

RUIZ MARIA TERESA DR

OBS ASTRON NACIONAL

UNI VERSIDAD DE CHILE

CASILLA 36-D

SANTIAGO

CHILE 
RULE BRUCE H STAFF RETIRE HALE URSERVATORIES

22.5 MCNTE VISTA ST

PASADE

U.S.A.

RUPRECHT JAROSLAV DR ASTRCNOMICAL INST OF HULECSKA $C$

CLECHCSLOVAK IA

RUSS LL JANE L CR

DLLE. THENY OBS

PITTSHURGH PA 15214

U.S.A.

RUSSC GUIDO DR

CSSE RVATORIO ASTRO-

NCNICO CAPODIMONTE

VIA NOIARELLO 16

$1 \% 3131$ NAPOL

RUSU L DR

ASTRENCMICAL OBS

5 CUTITUL DE ARGINT

EUCAKEST 28

RUZICKCVA TOPOLOVA

ELAZENA DR

ISTRCNOMICAL INST

CZECHOSLOVAK ACAD SC

251 I 5 CNDREJOV OBS

CZECHOSLOVAK IA

RYCKA EUGENIUSZ PROF DR

UL SZOPENA 23
30047 KRAKOW

PCLANO

FYCGREN ALFRED ERIC JR DR PHYS ICS CEPT

RENSSELAER POLYTECHN

INSTITUTE

TRCY NY 12180

U.S.A.

RYTEK CHARLES E DR

CEN SACLAY

DFH EP A

FF 2 I 190 GIF $S$ YVETTE

FRANCE

$S A A R$ EAN OR

DESERVATORY 5-19

2.2444 TORAVERE

ESTCNIA

U.S.S.R.

SACK NCAM DR

DEPT OF THEORET ICAL

HEPREW UNIVERSITY

JERUS ALEM

ISRAEL

SADLER DONALD H OR

8 CELLINGTON RISE

BEXHILL ON SEA

SUSSEX TN39 $3 R T$

U.K.
RUMSEY NORMAN J

PHYSICS AND ENG INEE-

RING LABORATORY

O S I R

LOWER HUTT

NEW ZEALAND

RUSCONI LUIGIA DR

$\checkmark$ IA GHIRLANDAIO $22 / 5$

$1-34100$ TRIESTE

ITALY

RUSSELL JOHN A PROF

DEPT OF ASTRONOMY

UNIV OF SOUTHERN

LOS ANGELES CA 90007

U.S.A.

RUST DAVID M DR

AMER ICAN SC IENCE AND

ENGINEER ING CO

955 MASS AVE

CAMBR IDGE MA 02139

U.S.A.

RUTTEN ROBERT JELLE DR

OBS SONNENBORGH

ZONNENBURG 2

3512 NL UTRECHT
THE NETHERLANDS

RYABOV YU A PROF DR

MATHEMATICS DEPT OF

MAOI

U. 25319 MOSCOW

RYBKA PRZEMYSLAW DR

INSTI TUTE OF HISTORY

DF SC IENCE

NOWY SWIAT 72

OC- 330 WAR SZAWA

POLAND

RYLE MARTIN PROF SIR

MADINGLEY ROAD

CAMBR IDGE CB 3 OHE

U.K.

RYZHKOV N F DR

SPECIAL ASTROPHYS-

OBS USSR ACADEMY SCI

LEN INGRAD BRANCH

196140 LENINGRAD

U.S.S.R.

SABAND YUTAKA DR ASTRONOMICAL INST TOHOKU UN IVER I TY ARAMAKI

SENDAI 980

JAPAN

SACKMANN I JULIANA DR

KELLOGG RADIATION

$\angle A B O R A T O R Y$

CALIF INST OF TECHN

PASADENA CA 91125

U.S.A.

SADZAKOV SOF I JA DR

ASTRONOMICAL OBS

VOLGINA 7

11050 BEOGRAD

YUGOSLAVIA
RUNCORN S K PROF

THE UNI VER SHY ICS

NEWCASTLE UPON TYNE

NEI $7 R U$

U.K.

RUSKOL EUGENIA L DR

OJSCHMIDT INSTI TUTE

OF PHYSICS OF EARTH

$A C$ SCI USSR

123810 MOSCOW

U.S.S.R.

RUSSEY RUSCHO DR

DEPT OF ASTRONOMY

ANTON IVANOV ST 5

IILLG SOFIA

RUSU I DR

ASTRONOMICAL ORS

5 CUTITUL DE ARG INT BUCAREST 28

ROUMANIA

RUZDJAK VLADIMIR DR

INST OF PHYSICS

UNIVERSI TY

$P$
$4100 B O X$ Z 304
YGREB

YUGOSLAVIA

RYBICKI GEORGE B DR

HARVARD SMITHSONI AN

C TR F ASTROPHYSICS

GO GAROEN ST

CAMBRIDGE MA 02138

U.S.A.

RYDBECK OLOF E H PROF

ONSALA SPACE

OB SERVATORY

S-430 34 ONSALA

SWEDEN

RYLOV V S DR

CAL IAL ASTROPHYSI-

USSR ACADEMY OF SCI

357140 N ARKHYZ

U.S.S.R.

RZHIGA $N$ DR

INSTITUTE OF RADIO

AND ELECTRONICS

USSR ACADEMY OF SCI

U.S.S.R.

SABBADIN FRANCO DR

OSSERVATORIO ASTROFISICO

I 36012 ASIAGO

SADEH D DR

DAPT OF PHYSICS AND

TEL AVIV UNIVERSITY

I SRAEL

SAFRONDY VICTOR S DR

INSTITUTE OF PHYSICS

OF THE EARTH

B GRUZINSKAYA 10

123242 MOSCOW

U.S.S.R. 
SAGA Y CARL DR

CCRNELL UNIVER ITY

ITFACA NY 14853

U.S.A.

SASNIER JEAN LOUIS MR

77 AURE DU LONGITURES

TCCHEREAU

$F=75,14$ PARIS

FRANCE

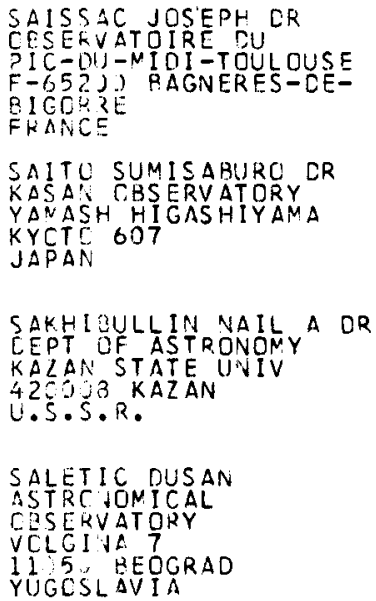

SALPETER EDWIN E PROF

VEWMAV LAE OF NS
CCRNELL UNIVERSITY

ITHACA NY 14853

U.S.A.

\section{SANAIN D DR \\ LFSP \\ P NC 10 \\ QUISSON}

SANCHEL FRANCISCO PROF

IACTITUTO ASTROFISI-

CA CANARIAS

UAIVFRSIDAD LAGUNA

LA LAGUNA TENER IFE

SPAIN

SANCIS I RENZO DK

KAPTFYN LAQORATORIUM

PCSTBUS 800

9700 RV GRONINGEN

THE NETHERLANDS

\section{SANUERS R DR \\ KAPTEYN LABORATORY \\ $P$ C PCX 800 \\ 97.0 RV GRON INGEN \\ THE NETHERLANDS}

SANCOVIST $\triangle A G E$ CR

STCCKHICLM

DPSERVATORY

$S-133$ OJ

SWLDEN JUEBADEN
SAGGION ANTONIO PROF ISTITUTO DI FISICA $G$ CAL ILE I VIA MARZOLO 8 IT 35100 PADOVA

SAHADE JORGE PROF 49342

1900 LA PLATA

AR GEN TINA

SAITO KUNIJI PROF TOKYO ASTRONOMICAL OQSER VATQRY

OSAWA MI TAKA

TOKYO 181

JAPAN

SAKASHITA SHIRO PROF

DEPT OF PHYSICS

HOKKAIDO UNIVERSITY

KITAJYUJO NI SHI 8

SAPPORO HOKKAIDO 063

JAPAN

SAKURAI KUNITOMO PROF

KANAGAWA LNIVERSITY

KANAGAWAKU

YOKOHAMA 252

J AP AN

SAL ISPURY $J W D R$

AFCRL

BLDG 1121

B G HANSCOM FIELD

U.S.A.

SALUKVADLE G $N$ DR ABASTUMANY ASTROPHYSICAL OBSERVATDRY 383762 ABASTUMANY

U.S.S.R.

SAMPSOY ROUGLAS H PROF DEPT OF ASTRONDMY

PENN STATE UNIV

525 DAVEY LAB

UN IV PARK PA 16802

U.S.A.

SANCHEZ MAGRO C DR

ASTROF IS ICA CANARIAS

UNIV DE LA LAGUNA

TENER IFE

SPA IN

SANDAKOVA E $V$ DR

KIEV STATE UNIVERSI TY ASTRONOMICAL

QBSERVATURY

$252053 \mathrm{KIEV}$

U.S.S.R.

SANDERS WL PROF

NEW MEXICD STATE

UN IVERSITY

AOX 4500

LAS CRUCES NM 88003

U.S.A.

SANDULEAK N DR

WARNER AND SWASEY

OBSERVATORY

1975 TAYLOR RD

E CLEVELAND OH 44112

U.S.A.
SAGI TOV M U DR

STERNBERG STATE AS-

117234 MOSCOW

U.S.S.R.

SAHAL-BRECHOT SYLVIE DR

OBSERVATOIRE DE

PARI S-MEUOON

FRANCE

SAITO MAMORU DR

OBSERVATORY

OSAWA MI TAKA

TOKYO 18

JAPAN

SAKHAROV VLADIMIR I OR

PULKOVD OBSERVATORY

196140 LENINGRAD

U.S.S.R.

SAKURAI TAKEO T PROF

FACULTY OF

ENG INEER ING

KYOTO UNIVERS ITY

SAKYO-KU KYOTO 606

JAPAN

SALOMONOVICH A E DR

PHYSICAL INSTITUTE

USSR ACADEMY OF SCI

$117924 \mathrm{M}$

U.S.S.R.

SAMAHA A H M PROF

5 WADI EL NILE ST

MAADI CAIRO

ARAB REP. OF EGYPT

SANAMIAN $V$ A DR

BYURAKAN ASTROPHYSI-

CAL OBSERVATORY

378433 ARMENIA

U. S. S.R.

SANCHEZ MANUEL

INSTI TUTO Y OBSERVA-

TORIO DE MARINA

SAN FERNANDO CADIZ
SPAIN

SANDELL GORAN HANS L DR STOC KHOLM OBS

S-13300 SALTSJOBADEN SWEDEN

\section{SANDF ORD MAXWELL T II \\ LOS ALAMOS SCI \\ LOS ALAMOS NM 87545 \\ U.S.A.}

SANFORD P W MR

MULLARD SPACE SC LAB

HOLMBURY ST MARY

DORKING

SURREY RHS SNS.

U. K. 
SANT IN PACLO DR IA CUCOVICH 3

SAPAR $\triangle R V E D$ DR

I APHA ESTONIAN AC OF

SCIENCE TORAVERE

U. U. $24.4 . T .2$.
.

SARGENT WALLACE LEW . CR.
HALE OBSERVATORIES

CALTECH

I2O I E CALIFORNIA ST

PASAEENA CA GII25

U.S.A.

SARRIS EMMANUEL T PH D

TERCC

XANTH

GREFCE SASLAW WILLLAM C PROF

UNIVERSITY VIRGINIA

RCX 3918 UNIVSTATION

CFARLOTTESV VA 22903

SATC FUMITAKA PROF

INST ITUTE OF FUNCA-

NENTAL PHYSICS

UAIVERSITY DF KYOTO

SAKYUKU KYOTU 606

JAPAN

SATC NACNOBU DR

AKITA UIO

JAPAN

\section{SAUVAL JACQUES CR \\ 3 AVE CIRCULAIRE \\ I-IISO BRUXELLES \\ C.ELÓIUM}

SAVEOUFF MALCOLM P PROF

PFYS AIV ASTRDN CEPT

LNIVERSITY ROCHESTER

BAUSCH ANO LOME ELDG

RCCHEST TR NY 14627

U.S.A.

SAXENA P P DR

UT T $\triangle R$ PRADESH

STATE CBSERVATOFY

MAICRA PEAK

WAINI TAL

INEIA

SCALTKITI FRANCO CR

CSSERVATOKIO ASTRO-

Nivi ICO

PINC IORIIIESE

TCi I

SCARRUTT STANLEY M CR
PHYSICS DEPT

UN IV CF DURHAM

SCUTH HCAE

DURHAM DHI 3LE

U.K.
SANWAL N B DR

DEPT OF ASTRONOMY

OSMANIA UNIVERSITY

HYDER

INDIA

SARAZIN CRAIG L DR

UNIV OF VIRGINIA

POB 3818 UNIV STA

CHARLOTTESVL VA22903

U.S.A.

SARMA M B K PROF

DEPT OF A STRONOMY

DSMANIA UNIVER SI TY

HYDERABAD 500007

INOIA

SARTOR I LEO PROF

BEHLEN LABORATORY OF

PHYSICS

UN IVERSITY NEBRASKA

LINCOLN NB 68588

U.S.A.

SASTRY CH V

ASTROPHYSICAL

OBSER VATORY

KOOA IKANAL 3

INDIA

SATO KATSUHIKO DR

RESEARCH INST FDR

FUNDAMENTAL PHYSICS

KYOTO UN I VERSI TY
SAKYOKU KYOTO 606

JAPAN

SATO SHUJ DR

DEPT OF PHYSICS

UN IVER SITY OF KYOTO

KITASHIRAKAWA

SAKYOKU KYOTO 606

JAPAN

SAVAGE ANN DR

ROYAL OBSERVATORY

BLACKFORD HILL

EDINBURGH EH9 $3 \mathrm{HJ}$

U.K.

SAWYER CONSTANCE S DR NOAA PMEL

3711 15TH $\triangle V E$ NE

SEATTLE WA 98105

11.5 .4 .

SCALISE JR EUGENIO DR

CRAAM-ON-CNPQ

OI243 SAO PAULO

ARAZIL

SCARFE COLIN D DR

DEPT CF PHYSICS

$P$ C BOX 1700

VICTOR IA BC V8W $2 Y 2$

CANADA

SCHADEE AERT DR

STERRE NACHT

SONNENEORGH

UTRECHT 2501

THE NETHERLANDS
SANYAL ASHIT DR

DEPT OF ASTRONOMY

PENNA STATE UNIV

U.S.A.

SAREYAN J P DR

OBS DE NICE

F-36007 NICE CEDEX

FRANCE

SARRIS ELEFTHERIOS PH D

NAT OBS OF ATHENS

ASTRONOMICAL INST

ATHENS 306

SASAO TETSUO DR

INTERNATI ONA

LATITUDE OBSERVATORY

OFF MIZUSAWA

MI ZUSAWA IWATE. 023

JAPAN

SATO FUMIO OR

CHIRA PREFECTURE

EDUCATI ON CENTER

1 O-I KATSURAGI 2-CHO

CHIBA 280

JAPAN

SATO KOICHI DR

INTERNATIONAL

LATI TUDE OBSERVATORY

OF MI ZUSAWA

MIZUSAWA IWATE 023

SATO YUZO DR

OSAWA 4-8-19

MI TAKA

TOKYO 181

JAPAN

SAVAGE BLAIR D DR

DEPT OF ÁSTRONOMY

UNI VERSITY WISCONSIN

475 N. CHARTER STR

U.S.A.

SAWYER HOGG HELEN B DR

DAVID DUNLAP DBS

$P$ O BOX 360

RICHMOND HILL

CNT L4C 4 Y6

CANADA

SCALO JOHNMICHAEL OR

UNIV DF TEXAS

AUSTIN TX 78712

U.S.A.

SCARGLE JEFFREY D DR

NASA-AMES RESEARCH

CENTER MS245-3

MOFFETT FIELD

$C A 94035$

U.S.A.

SCHAIFERS KARL DR

LANDESSTERNWARTE

KCENI SSTUH

D-6900 HEIDELBERG 1

GERMANY, F.R. 


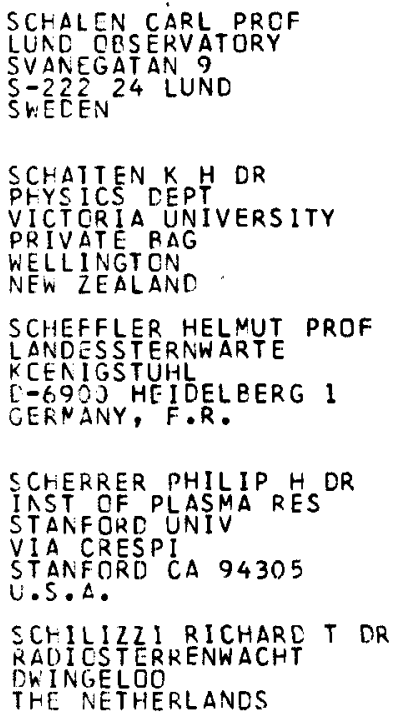

SCHKOOROV V G DR

DEPT OF ASTRONOMY 7 TH NOVEMBER ST 1 SCF I A

PULGARIA

SCHLUETER A PROF DR MPI F PLASNAPHYSIK $D-8: 46$ GARCHING GERMANY, F.R.

SCHMAHL EOWARD J DR ASTRCNOMY PROGRAM UAIV CF MARYLAND CCLLECE PK MD 20742 U.S.A.

SCHNEIDLER F PROF DR INST ITUT F ASTRONONIE UND ASTROPHYSIK SCHE INERSTR I D-8000 MUENCHEN 80 GERMANY. F.R. SCHNIDT H U DR ${ }^{H}$ PU FUER PHYSIK UND ASTRCPHYS IK FCEHKINGER RING 6 GERMSNY, F.R.

SCHMIOT MAARTEN PROF HALE OESERVATORIES 813 SANTA BAREARA ST PASAUENA CA 91101 U.S.A.

SCHNIEDER B DR FPS CE NEUDON FRANCE
SCHANDA ERWIN PROF

INST OF

SIDLER STR 5

CH-30I2 BERN

SWITZERLAND

SCHATZMAN EVRY PROF OBSERVATOIRE DE NICE BP 252 F-06007 NICE CEDEX FRANCE

SCHEIDECKER JEAN-PAUL DR DBSERVATOIRE DE NICE BP 252 F-O6OOT NICE CEDEX

SCHEUER PETER A G DR CAVENDISH LABORATORY MADINGLEY RO

CAMBR IDGE CB 3 OHE

$U \cdot K$.

SCHILLER KARL PROF DR PIR SCH-WEG 6 D-6072 DREIEICHBUCHSCHLAG

SCHLESINGER BARRY $M$ DR SYSTEMS AND APPLIED 681 I KEN I LWORTH.AVE RIVERDALE MD 20840 U.S.A.

SCHLUETER DIETER PROF INST THEO PHY STERNW NEUE UNIV PHYSIK ZTM OL SHAUSENST GB N GIC DE 2300 KIEL GERMANY, F.R.

SCHMAHL GUENTER PROF UN IVER S I TAE TSSTERNWARTE

GE ISMAR LANDS TR 11

O-340O GOETTINGEN GERMANY, F.R .

SCHMID BURGK J DR MPI RADIOASTRONOMIE AUF DEM HUECEL 69 $D-5300$ BONN 1 GERMANY, F,R.

SCHMIDT HANS PROF UN IVER SI TAE TSAUF DEM HUECEL 71 D-5300 BONN I

SCHMIDT THOMAS DR MPI FUER ASTRONOMIE KOENIGSTUHL D- 9900 HE IDELBERG GERMANY, F.R.

SCHMITTER Y MARTIN DEL CAMPO E F DR INST ASTRONOMY UNAM MEXICO D MFXICO
SCHARLEMANN ERNST T DR DEPT OF ASTRONOMY COLUMB I A UNIV

BOX 98 PUPIN

NEW YORK NY 10027

U.S.A.

SCHEEPMAKER ANTON DR COSM RAY WORK GROUP HUYGENS LABORATORY

WASSENAARSEWEG 78

2300 RA LE IDEN

THE NETHERLANDS

SCHERB FRANK PROF

PHYSICS DEP

UNI VERSI TY WISCONS IN MADISON WI 53706 U.S.A.

SCHILD RUDOLPH E DR

CTR F ASTRDPHYS ICS

60 GARDEN STREE

CAMRRIDGE MA O2138

U.S.A.

SCHILT JAN DR

188 HILLSIDE AVE LEQNIA

U. S.A.

SCHLOSSER WOLFHARD PROF ASTRONDMI SCHES

INSTITUT

POSTFACH 102148

D-4630 BOCHUM

SCHMADEL LUTZ D DR

ASTRONOMI SCHES

RECHEN-INSTI TUT

MOENCHHOF STR I $2-14$

GERMANY, F.R.

SCHMALBERGER DONALD C DR DUDLEY OBSERVATORY

1202 TROY-

SCHE NEC TADY RD

LATHAM NY 12110

U.S.A.

SCHMIDT EDWARD G

UNI VERSITY OF

NEBRASKA

LINCOLN NB 68588

U.S.A.

SCHMIDT $K H$ DR

ZNTR INST ASTROPHYSIK

STERNW BABELSBERG

ROSA LUXEMBURGST 17 A

DDR-1502 POTSDAM

G.D.R.

SCHMIDT-KALER TH PROF

STEINHUEGEL 105

D-58ID WI TEN

SCHNELL ANNELISE DR

INST FUER AS TRONOMIE

UNI VERSITAT WIEN.

TUERKENSCHANZST 17

$A-1180$ WIEN

AUSTRIA 
SCHNCPPER HERBERT W OR CTR FOR ASTROPHYSICS 60 GAROEN ST

CANEKIEGE MA 02138

U.S.A.

SCHCEFFEL EBERHARD F DR ASTRENOM ISCHES INST

RUHR UNIVERS ITAET

PCSTFACH 1021218

UE -463, BOCFUM

SCHCCNEICH W OR

ZNTRINST ASTROPHYSIK

ASTRGIP OBSERVATOR IUM

TELEGRAF ENBERG

C.C.R-15 PETSDAM

G.C.R.

SCHOLZ GERHARD CR

ZENTR INST ASTROPHYS

DER ADW DER DCR

RCSA-LUXENAURGST 17 A

CLR- 1502 POTSCAM

G.C.R.

SCHCCLMAN STEPHEN A DR

LCCKHEED PA RES LAB

3251 HANOVER ST

PALC ALTO CA 94304

U.S.A.

SCHROEDER DANIEL I PRDF

DEPT PHYS AND ASTRON

EELCIT COLLEGE

BELCIT WI 53511

U.S.A.

SCHRULL ALFRED DR

SCANENCBSERVATORIUM

KANZELHCEHE

$A-9520$ SATTENDORF

AUSTRIA

SCHUKART JOACHIM OR

ASTRDNOM I SCHES

RECHEN INSTITUT

NCENCHHOFSTR 12-14

$D-69,3$ HEI DEL BERG

GERNANY, F.R.

SCHUERNAN DONALD W CR

SPAC

EXECUTIVE PK EAST

DLBAAY NY 12203

U.S.A.

SCHUNANN JOERG OIETER DR

OBS HOHER L IST

UAIV STERNWARTE BONN

GERNANY, F.R.

SCHWAN HEINER: DR

ASTRCNCM ISCHES

RECHEN INST ITU

NCENCHHOFSTR I 2-14

D-6900 HEIDELBERG

GERMANY, F.R

SCHWARTZ RICHARE D

ASST PROF DEPT PHYS

$800 I$ NAT BRIDGE RD

ST LCUIS MO 63121

U.S.A.
SCHNUR G F 0

ESO ADMINISTRATION

ALONSO CORDOBA 3107

VITACURA CAS I I P

CHILE

SCHOEMBS ROLF UR

INST FUER ASTRONOMIE

ASTROPHYSIK

SCHE INER STR I

O-BOOO MUENCHEN 81

SCHOENFELDER VOLKER DR

MP I F EXTRATERRE-

STR I SCHE PHYSIK

D-8046 GARCHING

GERMANY, F.R.

SCHOLZ M PROF

INST F THEORETISCHE

ASTROPHYS DER UNIV

NEUENHE IMER FELD 294

D-6900 HE IDELBERG

GERMANY, $F \cdot R$.

SCHRAMM DAVID N PROF

UN IVER SI TY C

933 56TH ST

CHICAGO IL 60637

U.S.A.

SCHROEDER ROLF DR HAMBURGER STERNWARTE GD JENBERGSWEG 112 D-2O5O HAMBUR

SCHR UETER EBERHARD DR INST F ASTROPHYSIK UN IVER SI TAET BONN AUF DEM HUEGEL 71 $D-5300$ PONN 1

GERMANY, F.R.

SCHUECKING E L DR

DEPT OF PHYSICS

NEW YORK UNIVERSI

$U \cdot S . A$.

SCHULER WALTER DR

STERNWAR TE DER

KAN TON S SCHULE

CH-4500 SOLOTHURN

SWITZERLAND

SCHUSTER HANS-EMIL PH D EUROPEAN SOUTHERN OB CAL 16317 CORREO 99 SANTIAGD

CHILE

SCHWARTI DANIEL A DR CENTER FOR ASTROPHYS GO GARDEN ST CAMBR IDGE MA 02138 U.S.A.

SCHWARTZ ROLF PH D MP I FUER

RADIOASTRONOMIE

AUF DEM HUEGEL 69

D-5300 BONN 1

GERMANY, F.R
SCHOBER HANS J DR

ASTRONOMISCHES INST

UNI VERSI TAETSPLATZ 5

$A-8010$ GRAZ

AUSTRIA

SCHOENBERNER DETLEF PH D

INST F THEOR PHYSIK

OLSHAUSENSTRASSE

D-230O KIEL

GERMANY, F.R.

SCHOLL HANS OR

ASTRONOMISCHES

RECHEN-INSTI TUT

MOENCHHOF STR I2-14

D-6900 HEI DELBERG

GERMANY, F.R.

SCHOMBERT JOHN L MR

21920 DAVIS MILL RD GERMANTOWN MD 20767

U.S.A.

SCHRI JVER J DR

LABORATORIUM VOOR

RUI MTEONDERZOEK

$35 N E$ LUXLAAN 21

THE NETHERLANDS

SCHROETER EGON H PROF

KIEPENHEUER INST

FUER SONNENPHYS I K

SCHOENECKSTRASSE 6

GERMANY, F.R.

SCHRUTKA RECHTENSTAMM

GUNTRAM PRÖF DR

COTTAGEGASSE 56

A-I I 90 WIEN

SCHUERER MAX PROF

ASTRONOMI SCHES INST

UNI VERSITAET BERN

SIDLERSTR 5

CH-3012 BERN

SCHULTE D H DR

IOEK CCORPQRATION

LEXING TON MA O2173

U.S.A.

SCHUTZ BERNARD F DR

APPL IED MATHEMATICS

AND ASTRONOMY

UNI VER ITY COLLEGE

CARDIFF CFI IXL

. K.

SCHWARTZ PHILIP $R$ DR

NAVAL RESEARCH LAB

CODE 7138

WASHINGTON DC 20375

U.S.A.

SCHWARZ ULRICH J DR

KAPTEYN LABORATORIUM

POSTBUS 800

GRONINGEN

THE NETHERLANDS 
SCHWARZSCHILD MARTIN PROF PRINCETON UNIVERSITY

CBSERV ATORY

PEYTON HALL

PRINCETON NJ 08540

U.S.A.

SCCNZO PASQUALE DR

ARLINGTCN MA OZI74

U.S.A.

\section{SCCTT PAUL F CR \\ CAVENDISH LABORATORY \\ NADINGLEY ROAC \\ CANBRIDGE CB3 OHE \\ $U . K$.}

SEACUIST ERNEST R PROF UEPT OF ASTRONOMY

UNIVERS ITY

TCRCATO ONT MSS IAT

CANACA

SEATCN NICHAEL J PROF

UNIV CCLLEGE LONDON

GCWER ST

LCNDCN WCIE GBT

U.K.

SEINAK GIORGIO DR

CSSERVATORIO ASTRONO

NICE DI TRIESTE

VIA TIEPOLD II

ITALY

SEGAL IRVING E CR

$2-224$

CARBRIDGE MA 02139

U.S.A.

SEHNAL LADISLAV DR

ASTRCNCMICAL INST

CSAV

25165 CNDREJOV

CZECHOSLOVAK IA

SEIELSTAD GEORGE A

OWENS VALLEY RADIO

OHSERVATORY

PC PIOX $3 B 7$

EIC PINE CA 93513

U.S.A.

SEKI MUENZO DR ASTREVOMICAL INST

TCHCKU UNIVERSITY

KAWAUCHI

SENEA I 980

JAPAN

SENEL NEIR DR

DE NUUDCN

F-9 230 MLUDON

FRANCE

SEYGEUSCH KURT V CR $\triangle S T R Q P H Y S I K$

FCEHIOINGER RING 40

C. -8005 MUENCHEN 45

GERMANY, F.R.
SCHWEIZER FRANCOIS DR

CERTER-AMLOLICAN OBS

CASILLA 63-D

LA SERENA

CHILE

SCOTT ELIZABETH L PROF

STATISTICS LAB

UNIVERSITY OFF CALIF

367 EVANS HALL 320

U.S.A.

SCOVILLE NICHOLAS 2

ASTRO-PHYSICS DEPT

UN IVERSITY OF MASS

GRADUATE RES CEN

U.S.A.

SEARLE L DR

HALE DBSERVATORIES

PI3 SANTA BARBARA

U.S.A.

SEDOON HARRY MR

ROYAL OBSERVATORY

EDINBURGH EH9 3HJ

U.K.

SEEGER CHARLES LOUIS III

SAN FRANCISCO STATE

UN IVER S I TY

473 JAMES ROAD

PALD ALTO CA 94306

U.S.A.

SEGALUVITZ ALEXANDER DR 11 HABANIM ST

KEFAR SAVA

I SRAEL

SEIDELMANN P KENNETH DR US NAVAL OBSER VATORY

U.S.A.

SEITTER WALTRAUT C PROF

ASTRONOM I SHES INST

STE INFUR TER STR 107

D- 4400 MUENSTER/W

GERMANY, F.R.

SEK IGUCHI NAOSUKE DR

TOKYO ASTRONOM ICAL

OBSERVATORY

OSAWA MITAKA

TOKYO 181

JAPAN

SEMEN IUK IRENA DR

ASTRONOMICAL OBS

UL UJAZDOWSK IE

POLAND

SERKOWSKI KRYSZTOF DR

LUNAR AND PLANETARY

LABORATORY

UNIVER SITY AR I ZONA

TUCSON AZ 85721

U.S.A.
SCIAMA DENNIS W DR

DEPT OF ASTROPHYSICS

SOUTH PARKS ROAD

OXFORD OXI 3RQ

U. K.

SCOTT JOHN S DR

ASTRONOMY PROGRAM

UNIV OF MARYLAND

COLLEGE PK MD 20742

U.S.A.

SCUF LAIRE RICHARD DR INST D.ASTROPHYSIQUE UNI VERSITE DE LIEGE 5 A VENUE DE COINTE B-4200 COINTE-OUGREE BELGIUM

SEARS RICHARD LANGLEY DR DEPT OF ASTRONOMY

UNIV OF MICHIGAN

PHYSICS-ASTRO BLDG

ANN ARBOR MI 48109

U.S.A.

SEDLMAYER ERWIN DR

TI SCHE ASTROPHYSIK

NEUENHEIMER FELD 294

D-6900 HEI DEL

GERMANY, F.R.

SEEGER PHILIP A DR

LOS ALAMOS SCIENT I -

FIC LABORATORY

LOS ALAMOS NM 87545

U.S.A.

SEGGEWISS WILHELM DR

OB SERVATORIUM

HOHER LIST

D-5568 DAUN EIFEL

SEIDOV ZAKIR F DR

SHEMAKHA ASTRO-

PHYSICAL OBSERVATORY

373243 SHEMAKHA AZER

U.S.S.R.

SEKANINA ZOENEK OR

SMI THSONI AN ASTROPHY

SICAL OB SERVATORY

CAMBRIDGE MA O2138

U.S.A.

SELVELLI PIERLUIGI DR

VIA A TRONOMICO

VIA TIEPQLO IIIE
I TTALY 3 I TRIESTE
ITALY

SEN S $N$ DR

INDI AN ASSN FQR THE

CULTIVATION OF SC

JADAVPUR

INDIA

SERRANO ALFONSO DR

INST DE ASTRONOMIA

UNAM

APDO POSTAL 70-264

MEXICO 


\section{SERSIC J L DR \\ CESERVATOKIO \\ 5 COO CCRDUAA \\ $\triangle R G E N I$ INA}

SEVARLIC BRANISLAV M PROF

DEPT OF ASTRONOMY

UAIVERSITY BEOGRAC

VCLGINA. 7

11350 REOGRAD

YUGUSLAVID

SHAFFER DAVID B CR

CCEE 745

GREEABELT MD 20771

U.S.A.

SHAHPAZIAN RUMELIA K DR

QYUURAKAN ASTRO-

PHYSICAL OBSERVATORY

378433 BYURAKAN ARM

U.S.S.R.

SHAKHOVSKOJ N M CR

CRINEAN ASTROPHYSI-

CAL EBSERVATORY USSR

334413 CRIMEA

U.S.S.R.

SHALTOUT MESALAM A M DR

HELW AN OBSERVATIIRY

HELWAN

$A R A B$ REP. OF EGYPT

\section{SHAC CHING-YUAN \\ HARVARD COLLEGE \\ OESERVATORY \\ G. GARCEN STR \\ CANBHIDGE MA O2138 \\ U.S.A.}

SHAPIRC STUART L

ASST PROF

CRSK

CCRAELL UNIVERSITY

U.S.A.

SHARAF SH G DR

IASTITUTE OF THECRE-

TICAL ASTRONOMY

192197 LFNINGRAD

U.S.S.R.

SHAVER PETER A DR

KAPTEYN LABORATOR IUM

PCSTBUS 800

GRON INGEN

SHAW JCHN H PROF

$174 \mathrm{~W} 18 \mathrm{TH}$ AVE

CCLUNCUS CH 43210

U.S.A.

SHAWL STEPHEN J CR

DEPT OF PHYSICS AND

ASTRCNOMY

UAIVERSITY OF KANSAS

LAWRENCE KS 66045

U.S.A.
SERVAJEAN ROGER DR

OESERVATOIRE

DE MEUDON

F-92190 MEUDON

FRANCE

SEVERNYJ A B PROF DR

CRIMEAN ASTROPHYSI-

CAL OBSER VATORY USSR

ACAD OF SCI NAUCHNIY

334413 CR IMEA

U.S.S.R.

SHAH GHAN SHYAM A DR

INDIAN INST ASTROPHY

SAR JAP UR RD

KORAMANGALA

BANGALORE $560 \quad 034$

INDIA

SHAKESHAFT JOHN R DR CAVENDISH LABORATORY MADINGLEY ROAD

CAMBRIDGE CB 3 OHE

U.K.

SHAKURA N I DR

STERNBERG STATE AS-

TRONOM ICAL INSTITUTE

117234 MOSCOW

U.S.S.R.

SHANE C D ASTRONOMER

BOX 582

SANTA CRUZ CA 95061

U.S.A.

SHAPIRO IRWIN I PROF

54-620 MIT

CAMBR IDGE MA 02139

U.S.A.

SHAPLEY ALAN H

BOAA DER CO 80302

U.S.A.

SHAROV A S DR STERNBERG STATE ASTRONOMICAL INSTITUTE $117234 \mathrm{MOSCOW}$

U.S.S.R.

SHAVIV GIORA DR

TEL-AVIV UNIVERSITY RAMAT-AVIV

TEL $-A V I V$

SHAW R WILLIAM PROF CORNEL UN I VERSITY

IOS HALCYON HILL ITHACA NY 14850

U.S.A.

SHCHEGLOV P Y DR STERNBERG STATE ASIRONOMICAL IN

U.S.S.R.
SETTI GIANCARLO PROF LABORATORIO RADIOAST ISTITUTO DI FISICA VIA I RNERIO

ITALY 26 BOLOGNA

SEVILLA MIGUEL J DR ALMÁNSA 76

ID 20

SPAIN

SHAHAM JACOB PROF RACAH INSTITUTE OF PHYSICS

HEBREW UNIVERSITY

JERUSALEM GIOOO

I SRAEL

SHAKHBAZYAN YUR I J L DR PULKOVD OBSERVATORY 196140 LENINGRAD

U.S.S.R.

SHALLIS MICHAEL J DR

SOUTH PARKS ROAD

OXFORD OXI $3 R Q$

U. K.

SHANE $W W$ DR

STERREWACHT

POSTBUS 9513

2300 RA LEIDEN

THE NETHERLANDS

SHAPIRO MAURICE M LAB FOR COSMIC RAY PHYSICS CODE 7020 NAVAL RESEARCH LAB U.S.A.

SHAPLEY MARTHA B MRS 4170 PINON DR BOULDER CO 80303 U.S.A.

SHARPLESS STEWART PROF DEPT DF PHYSICS AND ASTRONOMY UNI VERSITY ROCHESTER ROCHES U.S.A.

SHAW J SCOTT DR ASTRONOMY UNI VERSITY GEORGIA ATHENS GA 30602 U.S.A.

SHAWHAN STANLEY D DR DEPT PHYSICS ASTRON

UNIV OF IOWA

U. S.A. CI TY IA 52242

SHCHEGLOV $Y P$ PROF DR ASTRONOMICAL INSTITU TE ULBEKIAN ACADEMY OF SCIENCES 700000 TASHKENT

U.S.S.R. 
SFCHECCLEV DIMITRIJ E OR PULKEVE OESERVATORY $19614 !$ LENINGRAD

U.S.S.R.

SHEELEY NEIL R DR

CCCE 7140 TVS

NAVAL RESEARCH LAB
WASHINGTON DC 20375

W.SSHINGT

SHEN CHUN-SHAN PRESIDENT

ASTRC SQCIETY CHINA

CEAN SCHCOL OF SC

NAT TS ING-HU
HS IN-CHU 300

TAIWAV

SHERIDAN K V CR

CIV IFR RADIOPHYSICS

CPING NSW

2121

AUSTRALIA

SHIRATA YUKIO OR

TIFIC NEASUREMENTS

TCHCKU UNIVERSITY

SENCAI 980

JAPAN

SHINILU MIKIO ASST PROF

IAST SPACE AERON SC

UNIVERS ITY OF TOKYO

TCKYC 153

$J \triangle P A N$

SHINCDA NAHIRO CR

TCKYC GAKUGEI
UAIVERSITY

AUKU IKIT AMACHI

KLGANEI TOKYO 184

JAPAN

SHIRYAEV A V CR

LEA INGRAD STATE UNI-

VERS ITY ASTRD

I9GI78 LENINGRAD

U.S.S.K.

SHCEEKCCK ROEERT R CR

CHATTERTOIN DEPT OF

ASTRCNOMY

UAIVERSITY OF SYDNEY

SYCNEY NSW 2006

AUSTRALIA

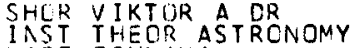

NAEEREZHNAYA

KUTUZOVA 1 ?

132137 LENINGR AD

II.S.S.R.

SHTEIUS K A DR

LATVIAN STATE UNIVER

SITY ASTRONOM I CAL

CBSERVATORY

226098 RIGA

U.S.S.R.

SHUK EA C S DR

FANAT RFSFARCH INST

P C HEPEAL

CAAGALCRE 560 OC6

INEIA
SHCHERBINA-SAMOJLOVA' I S

INSTITUTE OF SC IENCE

ANDN

I 25219 MOSCOW

U.S.S.R.

SHELUS PETER J DR

UNIVERSITY OF TEXAS

$R$ L $16-316$

AUSTIN TX 78712

U.S.A.

SHEN ZEE DR

DEPT OF MATHEMATICS

NATIONAL TAIWAN UNIV

TAIPEI

SHERWOOD WILLIAM A DR

MP I FUER RADIO

A STRONOMIE

AUF DEM HUEGEL 69

$D-5300$ BONN

GERMANY, F.R.

SHIELDS GREGORY A OR

RLM 15212

UN IV OF TEXAS

AUSTIN TX 78712

U.S.A.

SHIMIZU TSUTOMU PROF

DEPT DF ASTRONOMY

UNIVERS ITY OF KYOTO

SAKYOKU KYOTO 606

JAPAN

SHINE RICHARD A DR

NASA GSFC

CODE 685

GREENBELT MD 20771

U.S.A.

SHIVANANDAN KANDIAH DR NAVAL RESEARCH LAB

CODE 7122

WASHINGTON DC 20375

U.S.A.

SHOEMAKER E

US GEOLOGICAL SUR VEY U.S.A.

SHORE BRUCE W

LABORATORY

LIVERMORE CA 94550

U.S.A.

SHU FRANK H PROF

ASTRONOMY DEPT

UNIV OF CALIFORNIA

CAMPBELL HALL

BERKELEY CA $\$ 4720$

U.S.A.

SHUL MAN L M DR

MAIN ASTRCNOMICAL OB

SERVATORY UKRAINIAN

ACADEMY OF SCIENCES

$252127 \mathrm{KIEV}$

$U \cdot S \cdot S \cdot R$.
SHEA MARGARET A DR

AIR FORCE GEOPHYSICS

LAB PHC

HANSCOM AFB MA 01732

U.S.A.

SHEN BENJAMIN S P PROF

DEPT ASTRONOMY EI

UNI VERSITY OF

PENNSYLVANIA

PHILADELPHIA PAI 9104

U.S.A.

SHER PAVID DR

2837 MINTO DR APT ?

CINCINNATI OH 45208

U. S. A.

SHE VCHENKO V V DR STERNBERG STATE ASTRONOMICAL IN

117234 MOSCOW

U.S.S.R.

SHIM WOON-TAIK PROF

$236-53$ SINDAN-DONG

JOONG - KU

REPUBLIC OF KOREA

SHIMMINS A J

ASTRONONYY OBS

$P$ O BOX 276

PARKES NSW 2870

AUSTRALIA

SHIPMAN HENRY L DR

PHYSICS DEPT

UNIV OF DE LAWARE

NEWARK DE 19711

U.S.A.

SHKLOVSKY I S DR

INACE RESEARCH

USSR ACADEMY OF SCI

117810 MOSCOW

U.S.S.R.

SHOLOMITSKY G B DR

SPACE RESEARCH

INSTI TUTE

$117810 \mathrm{M}$

SHOSTAK G S DR

KAPTEYN ASTRON INST

POSTBUS 800

9700 AV GRDNINGEN

THE NETHERLANDS

SHUKLA K

DEPT OF MATHEMATICS

AND ASTRONOMY

LUCKNOW UNIVERSITY

LUCKNOW U P

I NDI A

SHUL'BERG A M DR OOE SSA STATE UNIVERSITY ASTRONOMICAL ORSERVATORY

270014 DDESSA

U.S.S.R. 
SHULCV O $S$ DR

LENINGRAO STATE UNI-

VERS ITY ASTRONOM ICAL

CESÉTIATCRY

I99178 LENINGRAD

U.S.S.R.

SICA DERRICK W PROF

TEPT CF PHYSICS

CARLETCN UNIVERS ITY

CTTALA KIS 5 R6

CANACA

SIEGLLELTET

GCETIIAGFN

AUPSINST $3-5$

D-34 SOETT INGEN

CERNAVY, F.R.

IILVEREERG ERIC C DR

UAIVERSITY OF TEXAS

ICX 1337

TERT DAVIS TX 79734

U.S.A.

SINKIN SUSAN M CR NT STRCNLO OESERVA-

$T C R Y$

CATEEPRA ACT 2606

AUSTRALIA

SINCN GEORGE W CR

CESERVATORY

SUNSPIT NM B8349

U.S.A.

SINCA NOFMAN R ASSOC PROF

ETIILEN LAB PHYSICS

U.S.CL

SINCN RENE L E PROF

INSTITUT

[ ASTRCPHYSIOUE

$P-42, C D$ CNTE-L IEGE

BELGIÚM

SINCNSCN S CHRISTIAN DR

1061 RUSSELL AVE

LCS ALTCS CA 94022

U.S.A.

SINCLAIR ANDREW T DR

ECYAL GREENWICH

OESERVATORY

HAILSHAM

EAST SUSSEX BN27 IRP

U.K.

SINVHIAL SHAMBHU OAYAL DR . KUNAUN UNIVERS ITY

NAINI TAL 263001

INIIA

SISSCN GEORGE M NR

PLANETREES WALL

U.K.
SHUTER WILLIAM L H DR

DEPT DF PHYSICS

UN IVERS ITY OF B C

2075 WESTBROOK MALL

VANCOUNE

SIOL ICHOVSKY MILOS DR ASAV

BUDEC SKA 6

12023 PRAHA 2

促

SILBERBERG REIN DR NAVAL RESEARCH LAB CODE 7028

WASHINGTON DC 20375

U.S.A.

SIM MARY E DR

RCYAL OBSERVATCRY

RLACKFORD HILL
EDINRURGH EHS $3 H \mathrm{JJ}$

U.K.

SIMMONEAU E OR

INST D ASTROPHYSIQUE

98 BIS BO ARAGO

F-7501 4 PARIS
FRANCE

SIMON J L MR

BUREAU D LONGI TUDES

77 AVENUE

F-7501 4 PAR IS

FRANCE

SIMON PAUL A DR

DE MEUDON

F-92 I9O MEUDON

SIMONENKO ALLA N DR

ASTRON COUNCIL

PYATN ITSKAYA 48

109017 MOSCOW

U.S.S.R.

SIMOVLJEVITCH JOVAN L DR

DEPT DF ASTRONOMY

FACULTY OF SC IENCES

STUDENTSKI TRG 16

11000 BEOGRAD

YUGOSLAVIA

SINNERSTAD ULF E PROF STOCK HOLMS S- 13300 SAL TSJUBADEN SWEDEN

SINZI AKIRA M DR HYDROGRAPHIC OEPT TSUKIJI 5 CHUO KU TCKYO 104 JAPAN

SISTERC ROEERTO F DR OBSERVATORIO

ASTRONOMICO

LAPR IDA 854

5 COO COR DOEA

ARGENTINA
SIHILLE FRANCOIS DBSERVATOIRE DE LYON F-69230 ST-GENIS LAVAL

FRANCE

SIEBER WOLFGANG PH D MPI FUE

RADI OASTRONOMIE

AUF DEM HUEGEL 69

D-5300 RONN I

SILK JOSEPH I PROF

ASTRONOMY DEPT

UNIV OF CALIFORNIA

CERKELEY CA 94720

U.S.A.

SIMEK MILOS DR

ASTRONOMICAL INST

CZECHO ACAD SCIENCES

ORSERVATORY

25165 ONDREJOY

CZECHOSLOVAKIA

SIMO CHARLES DR

PJE MARIMON 11

BARCELONA 21

SPAIN

SIMON MICHAL PROF

DEPT EARTH SPACE SC STATE UNIVERSITY OF

STOINY BROOK NY 11794 U.S.A.

SIMON PAUL C DR AV MANOIR D'ANJOU 14 B- II 50 BRUXELLES BELGIUM

SIMONS STUART DR
I 70 HOLMLEIGH ROAD
LONDON NIG 5 PY

U. $k$.

SIMS KENNETH P DR

SYDNEY OBSERVATORY

SYDNEY N S W 2000

AUSTRALIA

SINTON WILLIAM M

UNIV OF HAWAI I

2680 WOODLAWN DR

HONOLULU HI 96822

U.S.A.

SIRY JOSEPH W 43 W 42 ND WASHINGTON DC 20016

U.S.A.

SITARSKI GRZEGORZ DR CTR FOR SPACE RES

UL BARTYCKA 18

POLAND 
SITAIK G F DR CTERNEERG STATE ASE 117234 MOSCOW

U.S.S.R.

S JCGREN WILLIAM L OR JFL 264-738

480 STAK GROVE CRIVE PASAEIENA CA OIIU 3

U.S. A.

SKUNANICH ANDRE PRQF

IIICH ALI ITUDE

OESERVATORY

$P C$ POX 3000

PCULCER CD BO307

$U . S . A$.

SLEAARSKI TADEUSZ DR

UAIVFRSITY DOBS

CUCHANAN GAROENS

$K Y 16 \rightarrow L Z$

U.K.

SLCAIM E M DR

ASTRCNCMICAL INSTITU

T UZBEKIAN ACACEMY

CF SCIENCES

700030 TASHKENT

U.S.S.2.

SNEYERS PAUL PROF

$\triangle S T R C V O M I S C H$ INST

VAANSESTRAAT 61

P-3030 LEUVEN

BLLGIUN

SNITH ALEX G PRCF

CEPT PFYS

231 'SPACE SC PLCG

GAINESVILLE FL 32611

II.S.A.

SNITH ERADFORE A PROF

DEPT OF PLANETARY

SIENCES

UAIVERSITY ARIZONA

TUCSCA AL 85721

U.S.A.

SNITH CEAN F DR

GECPHYSICS

LINIV OF COLORADO

HCULEFR CO 80303

I.S.A.

SNITH GEOFFREY CR

DEPT CF ASTROPHYSICS

SCUTH PARKS ROAD

CXF (KI) CXI $3 R Q$

IJ.K.

SNITH HAYWOOD C OR

SEPT OF ASTRONOMY

UNIVEKSITY OF

TANPA FL 33620

U.S.A.

SNITU LINDSEY F DR

DEPT OF PHYS ICS

UNIV OF WOLLOVGONG

PE ECX 1144

WCLLCVGCNG NSW 2500

AUSTRALIA
SIVAN J P DR

LAB DIASTRON SPAT

TRAVER SE DU SI PHON

LES TROIS LUCS

F-13012 MARSE ILLE

FRANCE

SKALAFUR IS ANGELO

MATH RESEARCH CTR

NAVAL RESEARCH LAB

CASHINGTON DC 20375
U.S.A.

SLADE MARTIN A III DR

JPL

264-720

4800 OAK GROVE DRIVE

PASADENA CA 91103

U.S.A.

SLEE OPBOR

CS IRO

BOX 76

EPP ING NSW 2121

AUSTRALIA

SLYSH V I DR

SPACE RESEARCH

INSTITUTE

USSR ACADEMY DF SCI

117810 MOSCOW

U.S.S.R.

SMILEY CHARLES H DR

28 MONTAGUE ST

PROVIDENCE RI 02906

U.S.A.

SMITH ANDREW M DR

CODE 672 SPACE FLIGHT

CENTER

GREENBELT MD 20771

U.S.A.

SMITH ERUCE F DR THEORET $\&$ PLAN STUD $B R 245-3$

NASA-AMES RES CTR MOFFETT FLD CA 94035 U.S.A.

SMITH ELSKE V P DR UN IVERSI TY MAR YLAND

COLLEGE PK MD 20742

U.S.A.

SMITH HARCING E JR DR

PHYSICS DEPT C-OII

UNIV OF CALIFORNIA

SAN DIEGO

LA JOLLA CA 92093

U.S.A.

SMITH HENRY J DR

NASA HEACQUARTERS

CODE SG

WASHINGTON DC 20546

MEXICO

SMITH MALCOLM G DR

ANGLO AUSTRALIAN

OBSER VATORY

$P$ O BOX 296

EPPING NSW 2121

AUSTRAL IA
SIVARAMAN $K$ R DR I NDIAN INSTI TUTE OF ASTROPHYSICS BANGALORE 560034 IND I A

SKILLING JOHN DR PPLIED MATHS AND THEO PHYS SILVER STREET CAMBRIDGE CR3 SEW U.K.

SLAUCITAJS SERGEJS PROF DR OR SERVATORIO ASTRONO MICD DE LA PLATA 1900 LA PLATA ARGENTINA

SLETTEBAK ARNE PROF PERKINS DBSERVATORY DELAWARE OH 43015

U.S.A.

SMAK JOSEPH I PROF COPERNI CUS ASTRONOMI

CAL CENTER

UL BARTYCKA 18

OO 716 WARSAW

POLAND

SMIT J A PROF

PHYSICAL LAB

UNI VERSITY

SQRBONNE AAN 4

UTRECHT UITHOF

THE NETHERLANDS

SMITH BARHAM W DR

G-6 MS 665

LASL

LOS ALAMOS NM 87544

U.S.A.

SMITH CLAYTON A JR DR U S NAVAL OBS

WASHINGTON DC 20390

U.S.A.

SMITH F GRAHAM PROF

ROYAL GREENWICH

DBSERVATORY

HAI L SHAM

EAST SUSSEX BN27 IRP

U. K.

SMITH HARLAN J PROF

ASTRONOMY DEPT

UNIVERSITY OF TEXAS

RL MODPE HALL 15,206

U.S.A.

SMITH HUMPHRY M

23 NORMANOALE

BEXYI LL ON SEA SAS TL TH

U. K.

SMITH MYRON A ASST PROF

DEPT OF ASTRONOMY

UNI VERSITY OF TEXAS

AUSTIN TX 78712

U.S.A. 
SNITH: PETER L DR

CTR FOK ASTROPHYSICS OUARCEN ST

CANBRICGE MA O2138

U.S.A.

SNULLINKI JAN CR

NICA ICUS AST

$C+C P N A D 12=18$

Q7-1 1 TORUN

PCLAND

SNRIGLIO FILIPPO PROF

CSSFRVATORIO

$\triangle S T R C N C M I C D$

VIA PARCO MELLINI 84

I J) I 36 ROMA

SNCW THEODORE P PROF OEPT OF PHYSICS AND $\triangle S T R E P H Y S I C S$

UNIVERS ITY COLORACC

PCULDER CO 80309

U. S.A.

SCEIESKI STANLEY DR

CCEE 673

GCEEARE SPACE FLIGHT

CENTER

GREENBELT MD 20771

U.S.A.

SCrOLEVA NS DR

SPECIAL ASTROPHYS

OES LSSR ACAD OF SCI

LEVINGKAD BRANCF

19614.1 LENINGRAD

U.S.S.R.

SCLERBLOM LARRY LR

US GEOLOGICAL SURVEY

2555 U GEMINI DRIVE

FLAGSTAFF AL 86001

U.S..

SCIFER BARUCH T DR

32 PYYSICS DEPT

CALTECH

PASARENA CA 91125

U.S.A.

SCLHEIM JAN ERIK

INR P O BOX 953

NOG I TROMSO

NCRWAY

SCNETT CHARLES P PROF

DEPT PLANETARY SCI

UAIVTRSITY OF

$\triangle R I Z O N A$

TUCSCN AZ 85721

U.S.A.

SCRCCHENKO R L CR

PEYSICAL INSTITUTE

USSR ACA

117924 MOS COW

U.S.S.R.

SCUFFRIN PIERRE E DR \& 252

F-D 3377 NICE CECEX
SM ITH RORERT CONNON DR ASTRONOMY CENTRE UN IVERSITY OF SUSSEX BRIGHTON BNI $9 Q \mathrm{H}$ U.K.

SMOLKOV G YA DR

SIBIZMIR

$\mathrm{P} B 4$

664697 IRKUTSK 33

U.S.S.R.

SMYLIE DQUGLAS E DR

DEPT OF PHYSICS

4700 KEELE STREET DOWNSVIEW ONT MSJIP3 CANADA

SNYDER LEWIS E

DEPT DF ASTRONOMY

UNIV OF ILLINDIS

UREANA IL GIBOI

U.S.A.

SCBOLEV V V DR

LENINGRAD STATE UNIV ASTRONDMICAL OBS

199178 LENINGRAD

SOBOUTI YOUSEF PROF

B IRUN I OB SER VA TORY

AND PHYSICS DEPT

UN IVER S ITY

SHIRAZ

IRAN

SOFIA SABATINO PROF GODDARD SPACE FLIGHT CENTER

CODE 681

GREENBELT MD 20771

U.S.A.

SOLC IVAN DR

ASTRON INST CSAV

GROUP OF OPTICS

DVORAKOWA 298

511 OI TURNOV

SOLOMON PHILIP M DR ASTROPHYSICS PROGRAM DEPT EARTH SPACE SCS

SUNY AT STONY BROOK

STONY BROOK NY 11794

U.S.A.

SORENSEN GUNNAR DR

INSTITUTE OF PHYSICS

LANGEL AND SGADE

DK -8000 AARHUS C

DENMARK

SORU-ESCAUT IRINA MRS

OBSERVATOIRE

DE MEUDON

$F=92190$ MEUDON

FRANCE

SOULIE GUY

OBS DE LUNIVERSITE

DE BORDEAUX

F- 33270 FLOIRAC

FRANCE
SMITH WM HAYDEN PROF

MCDONNELL CENTER FOR SPACE SCIENCES

WASHINGTON UNIVERS IT

ST LOUIS MD 63130

U.S.A.

SMOLUCHOWSKI ROMAN PROF

ASTRONOMY DEPT

UNIVERSITY OF TEXAS

RLM-15-212

AUSTIN TX 78712

U. S. A.

SMYTH MICHAEL J DR

DEPT OF ASTRONOMY

ROYAL ORSERVATORY

EDINBURGH EH9 $3 \mathrm{HJ}$

U. K.

SOBERMAN ROBERT $K$ OR

FRANKLIN RES CENTER

THE FRANKLIN INST

2O+B. FRANKLIN PARKW

PHILADELPHIA PAI9IO3

U.S.A.

SOBOLEV VLADISLAV M DR

MAIN ASTRONOMICAL

OB SERVATORY PULKOVO

196140 LENINGRAD

U.S.S.R.

SOCHILINA ALLA S DR

INSTI TUTE FOR THEORE

TICAL ASTRONOMY

10 KUTUZOV QUAY

192187 LENINGRAD

U.S.S.R.

SOFUE YOSHIAKI DR

DEPT OF PHYSICS

UNI VERSITY OF NAGOYA

FUROCHO CHI KUSAKU

NAGOYA 464

JAPAN

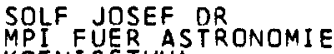

KOENIGSTUHL

D-6900 HEI DELBERG 1

GERMANY, F.R.

SOMERVILLE WILLIAM B DR UNIVERSITY COLLEGE

LONDON WCIE GRT

U.K.

SORENSEN SOREN-AKSEL DR

UNIV OF LONDDN

OBSERVATORY

MILL HILL PARK

LONDON NWT $2 Q S$

. $K$ 。

SOTIROVSKI PASCAL DR

OBSERVATOIRE

DE MEUDON

F-92190 ME UDON

FRANCE

SOUTHWORTH R $P$ DR

HAR VARD COLLEGE OBS

OO GARDEN STREET

U.S.A. 


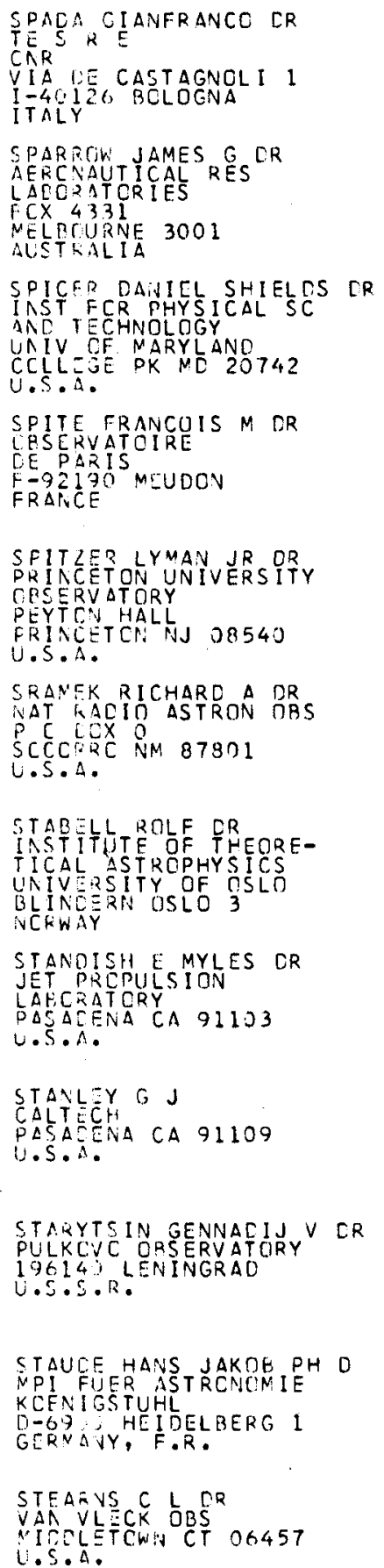

STAUCE HANS JAKOE PH D

NPI FUJER ASTRCNOMIE

KCENIGSTUHL

G-69 HEIDELBERG 1

STEAEYS C L RR

$\checkmark A A, V L E C K$ OBS

NICLLETCW CT O6457

U.S.A.

SPIEGEL E DR

COURANT INST

MATHEMATINAL SCS

251 MERCER STREET

U.S.A.

SPITE MONIQUE DR

OBSERVATO

FE PAR IS MEUDON

FRANCE

SPCELSTRA T A TH DR

STERREWACHT

2300 RA LE IDEN

THE NETHERLANOS

SREEKANTAN B V DR

TATA INST ITUTE OF

HOMI BHAEHA ROAD

BOMBAY 400กO5

INDIA

STAHR-CARPENTER M DR LEANOER MCCORMICK OE BOX 3918

UNIVERSITY STATION

CHARLOTTESVL VA22903

U.S.A.

STANILA GEORGE DR

ASTRONOMICAL OBS 5 CUTITUL DE ARGINT BUCAREST 28

ROUMANIA

STANNARD DAVID DR

JODRELL BANK

MACCLESFIELO I $90 L$

U.K.

STASINSKA G DR

OBS DE MEUDON FRANCE

STAUDE JUERGEN DR

ZENTRAL INST FUER

SOLAR - TERR PHYSIK

TELEGRAFENBERG

DDR - I5 POTSDAM

G.D.R.

STECHEP THEODORE $P$

CODE 672

GOODARO SPACE FLIGHT CENTER

GREENRELT MD 20771

U.S.A.

SPARKS WARREN M DR CODE 671 SPACE FLIGHT CENTER

GREENBELT MO 20771

U.S.A.

SPENCER RALPH E QR

NOMY LABS

JODRELL BANK MACCLES

FIELD CHES SKII 9 DL

U. K.

SPINRAD HYRON PROF

DEPT OF ASTRONOMY

UNIVERSITY

OF CALIFORNIA

BERKELEY CA 94720

U.S.A.

SPITHAS ELEFTERIOS $N$ DR

DEPT OF ASTRONOMY

PANE PISTIMIOPOLLIS

$\triangle$ THENS 621

GREECE

SPYROU NICOLAOS DR

DEPT OF ASTRONOMY

UNIVERSITY OF

THE SSALONIKI

THE SSALONIKI

GREECE

SREENIVASAN S RANGA PROF

PHYSICS DEPT

THE UNI VERSITY

2920-24 AVE N W

CALGARY ALTA T2N INA

CANADA

STALIO ROBERTO DR OSSERVATORIO ASTRONO MICO OI TRIESTE YIA TREPOLO II I TALY

STANKEVICH K S DR

RADI OPHYSICAL

603600 GORKIJ

U.S.S.R.

STARRFIELD SUMNER PROF DEPT OF PHYS ICS

ARI ZONA STA

UNI VERSITY

TEMPE AZ 85281

U.S.A.

STAUBERT RUDIGER DR A STRONOMISCHES INST. DER UNIV TUEBINGEN WALDHAUSERSTR 64 D-740O TUEBINGEN GERMANY, $F \cdot R$.

STAWIKOWSKI ANTONI DR ASTRONOMICAL CENTER UL CHOPINA $12-18$ 87-10J TORUN

POLAND

STECKER FLOYDW DR LAB FOR HIGH ENERGY ASTROPHYS 660 NASA GOODARO SPACE CENTER U.S.A. 


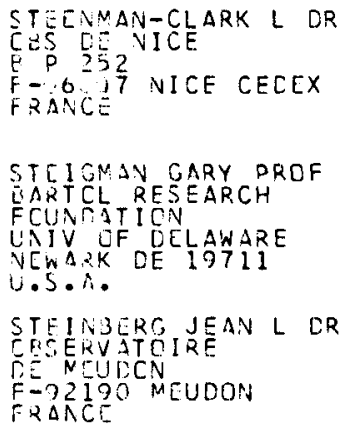

STELLINGWERF RORERT F OR

STEYAERT HERMAN PROF DR STERRENKUNDIG INST KRYGSLIAN 271 SO $B=909 J$ GEN

BELGIUM

SIIFT M J DR

IAST FUER ASTRONOMIE TUERKENSCHAN AUSTRIA

STCCK JURGEN

CEATRO DE INVESTIGACICV DE ASTRONOMIA $\triangle F A R T$ IDC 264 MER ICA VENEZUELA
STEFANOVITCH-GOMEZ A E DR OSSERVATOIRE

DE MEUDON

F-92190 MEUDON

FRANCE

STEIN ROBERT F ASSOC PRCF

ASTRONDMY DEPT

MICHIGAN STATE

UNIVER S ITY

EAST LANSING MI 48824

U.S.A.

STEINITZ RAPHAEL PROF PRYSICS DEPT UNIVERSITY OF THE

NEGEV

BEER SHEVA

ISRAEL

STELLMACHER GOET

INST D ASTROPHYSIQUE 98 BIS BO ARAGO

F -75014 PARIS

FRANCE

STEPANIAN A A DR

CR IMEAN ASTROPHYSI-

CAL OESERVATORY USSR

ACAD OF SCI NAUCHNIY

334413 CR IMEA

U.S.S.R.

STEPHENSON C BRUCE PROF WARNER ANO SWA SEY

BSERVATORY

1975 TAYLOR RD

E CLEVELAND OH 44112

STEPPE HANS OR

ASTRON INST BASEL

VENUSSTRASSE

CH-4ICZ BINNINGEN

SWITZERLAND

STESHENKO $N \vee D R$

CR IMEAN ASTROPHYSI-

CAL OBSERVATORY USSR

334413 CR IM.EA

U.S.S.R.

STEWART PAUL OR

MATHEMATICS DEPT

MANCHESTER MIIS $9 P L$

U.K.

STIBBS DOUGLAS $W N$ PROF UN IV OBSERVATORY

BUCHANAN GARDENS

ST ANDREWS

FIFE KYI6 $9 L 2$

$U . K$.

STIX MICHAEL DR

K IEP ENHEUER INST

SCHOENECKSTR 6

D-7800 FRE IBURG

GERMANY, F.R.

STOCKMAN HER VEY S JR DR

STEWARD OBS

UN IV DF AR IZONA

TUC SON AZ 85721

U.S.A.
STEIGER W R PROF

HAWAII INST GEOPHYS

UNI OF HAWAL

HONOLULU HI 96822

U.S.A.

STEIN WAYNE A PROF

SCHOOL OF PHYSICS

AND ASTRONOMY

UNI VERSI TY MINNESOT A

MINNE APOLIS MN 55455

U.5.A.

STEINLIN ULI PROF ASTRONOMISCHES INST UNI VERSITAET BASEL

VENUSSTR 7

CH-4 I 2 B INNINGEN

SWITZERLAND

STELLMACHER IRENE DR

BUREAU D LONGITUDES 77 AVENUE DENFERTROCHEREAU

F-75014 PARIS

FRANCE

STEPANIAN N N OR

CR I MEAN ASTROPHYS I-

ACAD DF SCI NAUCHNIY

334413 CRIMEA

$U . S \cdot S \cdot R$.

STEPHENSON F RICHARD DR

SCHOOL OF PHYSICS

UNIVERSITY

NEWCASTLE UPON TYNE

NE 1 TRU

U.K.

STERKEN CHRISTIAAN LEO DR

ASTROPHYSICAL INST

$\checkmark \cup B$

PLEINLAAN

B-1 OSO BRUSSELS

BELGIUM

STEVENS GERARD A DR

LABORATORI UM VOOR

RUIMTEONDERZOEK

BENE LUXLAAN $2 I$

UTRECHT

THE NE THERLANDS

STEWART RONALD T MR

RADI OPHYSICS

$P$ B BOX 76

EPP ING NSW 2121

AUSTRALIA

STICKLAND OAVID J OR VILLAFRANCA DEL CAST P OBOX 54065 MADRI D

SPAIN

STOBIE ROBERT S DR

INST OF ASTRONOMY

CAMBRIDGE CB3 OHA

U.K.

STOCKTON ALAN N DR

INSTI TUTE FOR

ASTRONOMY

2680 WOODLAWN DR

HONOLULU HI 96822

U.S.A. 


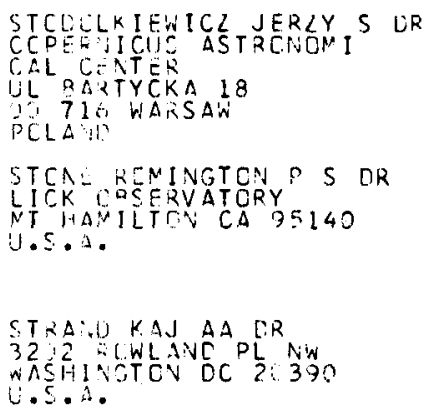

STREL'AITSKIJ VLAUIMIR OR ASTRLVCMICAL COUNCIL USSR ACAO OF SCS $1090: 7$ MOSCOW

U.S.S.R.

STRCOEL WILLI DR RECHEN INSTITUUT MUENCHHCFSTR 1214 (1)-6,9 HE IOELBERG 1 CERNAIY, F.R.

STRCN EICHARD G CR RAQI USTERRENWACHT
CUI: HCOGEVEENSETIJK

IWIVLELCO-NL 7514

THE VETHERLANDS

STHCNGKEN BENGT PROF

GANLE CAPLSBERCV 15

TK-253\% COPENHACEN

$\checkmark A L E Y$

CENNARK

STUNPFF PETER CR

NPI FUER R

AUF $=N$ HUESEL 69

CERNAYY, F.R.

SUCA KATUO ASST PROF

LCPT CF ASTRDNOMY

TEHCKU UNIVERSITY

AKANAKI

SUSAWA CHIKARA CK

INTERNATI ONAL LAT ITU

IIUSAWA

MIZUSAWA-IWATE 023

JAPS?

SULENT IC JACK W CR

WICHICHAN ST UNIV

F LANSING MI 48824

L.S.A.

SUNNCKS HUGH P CR 1)EPT OF APPLIED MATH AND THEORETICAL PHYS SILVER ST

CANEKICGE CB3 9EW

U.K.
STOHL JAN DR

ASTRONOMICAL INST

SAV

DL:PRAVSKA CESTA A 11

89930 ERATISLAVA

CZLCHCSLOVAKIA

STOYKO ANNA

OPSERVATOIRE

DE PAR IS

GI AVE DPSER VATOIRE

F-75014 PARIS

STRASSL HANS L PROF

DCHTRUPWEG 39

D-4AOC NUENSTER
GERMANY, F.R.

STRITTMATTER PETER A PROF STEWARD CB, SERVATORY TUC SON AZ BST21

U.S.A.

STROHMEIER WOLFGANG PROF VOLKFELDSTRASSE 5

D- B6CJ BAMBER

GERMANY, F.R.

STROM ROEERT G PROF

DEPT OF PLANETARY SC

UN IVER SI TY

OF AR IZONA

TUC SON AZ 85721

U.S.A.

STRONC IAN B DR

LOS ALAMOS SCI LAB

MAIL STOP 436

LOS ALAMOS NM 87545

U.S.A.

STURCH CONRAD R DR

1942 LEWIS AVE

ROCKVILLE MD 20851

$U . S . A$.

SUEMOTO ZENZABURO PROF DR TOKYO ASTRONONICAL

OPSER VATORY

OSAWA MITAKA

TOKYO 181

JAPAN

SUGIMOTO DAIICHIRO PROF

DEPT EARTH SC ASTRO

COLL GENERAL EDUC

UNIVERS I TY OF TOKYO

MEGURO

SULL IVAV WOODR UFF T IIIDR

UN IVER S ITY OF

WASHINGTON

SEATTLE WA 98195

U.S.A.

SUZUK I YCSHIMASA PROF

23-1 NAKAJ IMA

HIRONOMACH

UJI SHI 611

JAPAN
STONE R ${ }^{R} G$ DR

LAB FOR EXTRATERRES

PHYSICS NASA-GSFC

GREENBELT MD 23771

U.S.A.

STRAIZYS $V$ PROF OR

ASTRONOMIJOS OBS

VILNIUS 31

CIURLIONID 29

II THUANI A

U.S.S.R.

STRAUSS FEDERICO $M$ DR

CRAAM/ON/CNPQ

RUA PARA 277

OI 243 SAO PAULO SP

BRAZIL

STRDREL ANDRZEJ DR

INST OF ASTRONOMY

N COPERNICUS UNIV

CHOPINA $12 / 18$

87-1GS TORUN

POLAND

STROM KAREN M

KITT PFAK NATIONAL

OESERVATORY

$P$ O BOX 26732

TUC SON AL 85726

U.S.A.

STROM STEPHEN E

GALACTIC EXTRAG PROG

KITT PEAK NAT OBS

$P$ O $80 X 26732$

TUCSON AZ 85726

U.S.A.

STRONG JOHN D PROF

ASTRONOMY RESEARCH

FAC I LITY

UNI VERSITY OF MASS

AMHERST MA O1003

U.S.A.

STURROCK PETER A PROF INST PLASMA RESEARCH VIA CRESPI

STANFORD UNIVERSITY

STANFORD CA 94305

U.S.A.

SUESS STEVEN T DR

LABORATORY

NOAA ERL

BDULDER CO 80302

U.S.A.

SUKHAREV LEONID A DR

PULKOVOO OBSERVATORY

196140 LENINGRAD

U.S.S.R.

SULTANOV G F ACAD

SHEMAKHA ASTROPHYSI -

CAL OBSERVATORY

373243 AZERBAIDZAN

U. S. S.R.

SVALGAARD LEIF DR

INST FOR PLASMA RES

STANFORD UNIV

VIA CRESPI

STANFDRD CA 94305

U.S.A. 


\section{SVATOS JAN DR LCPT OF ASTRONOMY SVECSKD 8 \\ IS ÉFHSLOVAKIA \\ SVCLCPCULOS SOTIRIOS PROF \\ CEPT OF ASTROPHYSICS \\ UAIVERSITY OF ATHENS \\ ITHENS G21 \\ DREECE}

SWEET PETER A PREF
CEPT OF ASTRCNOMY
THEUUIVERSITY
GLKSOW GIZ BOW
U.K.

SWENSSEN JOHN W CR

SEELVEGATAN I4

SWE 252 LUNO

SWINGS JEAN-PIERRE CR

4 ISI NEITPE

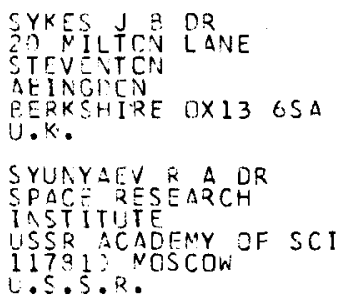

SZEQ OHELY VICTOR G PROF

UAPT AEROSPACE ENG

ULSTIVTX 78712

U.S.A.

TARARA HIRUTO OR

FACULTY OF EDUCATION

UTSU NQMIYA UNIV

$N$ INENACH] I

LISUNUMIYA 325

JAPA:

TACLIAFERH I CIUSEPPE PROF

CSSEKVATORIO ARCETRI

LARGL FERMI 5

ITALY FIRENZE

TAKACI SHIGETSUGU DR

INT KIATIONAL LAT ITU

QIZUSIWA IWATE 023

JAPAi,

TAKABALA KATSUO ER

KYCTC UNIVERSITY IND

BRIS TETILE FIFLRS

WATEUCASAKI SAKYCKU

K.YCTL C.)

JAPAS
SVECHNIKOV M A DR ASTRONOMICAL DEPT OF

STATE UN IVER SITY

620083 SVERDLOVSK

U.S.S.R.

SWANENBURG B N DR

COSMIE RAY WORKING

GROUP HUYGENS LAB

WASSENAAR SEWEG 78

THE RETHERLANDS

SWEIGART ALLEN V DR

CODE GODCARD SPACE

FL IGHT CENTER

GREENBELT MD 20771

U.S.A.

SWERCLOV NOEL PROF

UNIV OF CHICAGO

U.S.A.

SWINGS $P$ DR

AVEL SOUGUENET 23

B- 4050 ESNEUX

SYKES-HART AVR IL B DR

SYP ES OHAR TSAVRIILSICS

OXFORD OXI $3 R Q$

U.K.

SZABADOS LASZLU PH D KONKOLY OB SERVATORY $P$ BOX ET H-1525 BUDAPEST

HUNGARY

SZEIOL BELA OR

KENKOL Y OESERVATORY 1525 RUDAPEST

HUNGARY

TADEMARU F DR

UNIV OF MASS

AH'HERST MA 01002

U.S.A.

TAI YUIN-KWE I PROF

NO 7 CHAO-CHOU ST

TAIPEI

TAIWAN

TAKAKUHO KEIYA PROF

DEPT OF ASTRONOMY

TOHOKU UN I VER S I TY

ARAMAKI SENDAI 980

$J \triangle P A N$

TAKASE BURSSHIRO PROF TOKYO ASTRONOMICAL

OESERVATORY

OSAWA MITAKA

TOKYO 181

JAPAN
SVESTKA ZDENEK DR

SPACE RESEARCH LAB

BENE LUXLAAN 21

UTRECHT

THE NE THERLANDS

SWARUP GOVINO PROF

RADIO ASTRONOMY

CENTRE OF TIFR

POST BOX 8

OOTACAMUND 643001

INDI A

SWENSON GEORGE W JR PROF

OBSERVATORY

UNIVERSITY

OF ILLINOIS

URBANA IL 61801

U.S.A.

SWIHART THOMAS L DR

STEWARD ORSER

ARI ZONA

TUC SDN AZ 85721

U.S.A.

SWOPE HENRIETTA H DR

HALE OBSERVATORIES

PASADENA CA 91106

U.S.A.

SYKORA JULIUS DR

SKALNATE PLESO

ORSERVATORY

O59 6O TATRANSKA

LOMNICCA LOVAKIA

SZAFRANIEC ROZALIA DR

ASTRONOMICAL OBS

UL ORLA 171

$30244^{\circ}$ KRAKOW

POLAND

SZKOUY PAULA OR

DEPT OF ASTRONOMY

UNI V OF WASHINGTON

SEATTLE WA 98195

U.S.A.

TAFFARA SALVATORE PROF VIA CALZA 5 BIS

TAKAGI KOSHIRO PROF

DEPT OF PHYSICS

TOYAMA UNIVFRSITY

3190 GOFUKU

TOYAMA 920

JAPAN

TAKAKURA TATSUD PROF

DEPT OF ASTRONONY

UNIVERSITY OF TOKYO

EUNKYO-KU

TOKYO 113

JAPAN

TAKAYANAGI KAZUO PROF

INST SPACE AERON SC

UNIVERSITY OF TOKYO

KOMABA MEGURD KU

TOKYO I 53

JAPAN 
TAKECA HIDENOR I DR QEPT OF AERONAUT ICAL KYCTO UNIVERS ITY SAKYOKU KYOTO 606 JAPAN

TALBCT RAYMOND J JR PROF SPACE PHYSICS AND

RICE UNIVERS ITY

HCUSTCN TX 77001

U.S.A.

TANENAGA TATSUO OR

TACULTY OF EDUCATION

MIE UNIVERSITY

TSU SHI MIE 514

JAPAN

TANABE HIROYOSHI DR

TCKYC ASTRONOMICAL

CESFRVATORY

CSAWA MITAKA

TEKYC 181

JAPAN

TAAAKA RI ICHIRO PROF

FACULTY GENERAL ECUC

ASAHINACHIDOR I

NIIGATA 951

$J A P A N$

TANDEERG HANSSEN EINAR A

DR

NASA MARSHALL SPACE

FLIGHT CENTER ES5I

U.S.A.

TAPLEY BYRON D CR

CEPT AEROSPACE ENGR

ANO [NGR MECHANICS

UNIV TX-AUST WRW

U.S.A.

TARNSTROM GUY CR

NIT LINCOLN LAR

$P$ C BOX 73

LEXINGTCN MA 02173

U.S.A.

TASSCUL JEAN-LOUIS PROF

UNIVERSITE MONTREAL

CP 6128

CANTREAL PQ H3C 3J7

TATCN RENE PROF

KCYRE

I2 RUE COLBERT

$F=75002$ PARIS

FRANC

TAVARES J T L DR

$A V$ CIAS DA SILVA

17 R/C ESQ

CEIMBRA

TAYLER ROGER J PROF

ASTRCNOMY CENTRE

UA IVERSITY OF SUSSEX

CRIG

U.K.
TAKENOUCHI TADAO DR

I $-28-30 \mathrm{~K} I C H I J Y O J I$

MUSASHINO TOKYO 180

JAPAN

TALON R DR

CESR

9 AV DU COL ROCHE

B P 4336
F- $310299^{\circ}$ TOULOUSE CED
FRANCE

TAMMANN G ANDREAS PROF OR ASTRONOMI SCHES INST UN IVERS I TAET BASEL

VENUSST 7

CH-4IO2 B INNINGEN

SWITZERLAND

TANAKA HARUD PRDF

TOKYO ASTRONOMICAL

OBSERVATORY

OSAWA MITAKA

TOKYO 181

JAPAN

TANAKA YASUO DR

FACULTY OF EDUCATION

IBARAKI UNI VERSITY

BUNKYO

JAPAN

TANDON JAGDISH NARAIN DR

DEPT OF PHYSICS AND

ASTROPHYSICS

UN IVERSITY OF DELHI

DELHI I 10007

INDIA

TARAFDAR S P DR

HON I BHABHA ROAD

ROMBAY 400005

INDIA

TARTER C BRUCE DR

LAWRENCE LIVERMORE

LABORA TORY L-71

UNIVERSITY OF CALIF

LIVERMORE CA 94550

U.S.A.

TASSOUL MONIQUE OR

CIO DEPT DE PHYSIQUE

UNIV DE MONTREAL

MONTREAL P Q H3C 3J7 CANADA

UATUM JEREMY B DR TO-

RIA OBSER VATORY

VICTORIA BC VBW $2 Y 2$

CANADA

TAVASTSHERNA KIRILL N DR PULKOVO OESERVATORY 196140 LENINGRAD

U.S.S.R.

TAYLOR DQNALO J DR

ASTRONOMY

UN OF NEBRASKA

LINCOLN NB 68588

U.S.A.
TAKEUTI MINE DR

DEPT OF ASTRONOMY TOHOKU UNIVERSITY ARAMAKI SENDAI 980 JAPAN

TALWAR SATYA P DR PHYSICS DEPT

DELHI UNIVERSITY INDI A

TAMURA SHINICHI DR DEPT OF ASTRONOMY TOHOKU UNIVERSITY
ARAMAKI SENDAI 980 JAPAN

TANAKA KATSUD OR TOKYO ASTRONOMICAL OB SERVATORY

OSAWA MITAKA

TOKYO 1 BI

JAPA.N

TANAKA YASURO PROF

INST SPACE AERON SC

UNI VERSITY OF TOKYO

KOMABA MEGURO-KU

TOKYO 153

JAPAN

TANNER RICHARD

I046 F I SHER AVENUE

OTTAWA ONT

CANADA

TARENGHI MASSIMO DR

LABORATORIO DI FISI-

CA COSMICA

VIA CELORIA 16

I-20I33 MI LAND

I TALY

TARTER JILL C DR

MS 245-3

MOFFETT FLD CA 94035

U.S.A.

TATE VYAN S K DR

ASTRONOMICAL COUNCIL

PYATNITSKAYA UL 48

IOgOI T MOSCDW

U.S.S.R.

TAUBER GERALD E PROF

DEPT DF PHYSICS

TEL AVIV UNIVERSITY

IEL AVIV

TAWADROS MAHET JACOUB DR HELWAN OBSERVATORY

HELWAN-CAIRO
ARAB REP. OF EGYPT

TAYLOR GORDON E

ROYAL GREENWICH

OBSERVATORY

HAI L SHAM

EAST SUSSEX BN27 1RP

U. K. 
TAYLCR JCSEPH H PROF

UNIV VASSACHUSETTS

GRADUATE RES CTR

TCWEP 8

ANHERST MA 01003

U.S.A.

TEERIKORPI VELI PEKKA DR

CES ANC AST ROPH LAE

TAHT ITORN INMAK I

SF-0. 33 HELSINK I 13

FINLAND

TELEK! GEORGE OR

ASTRCVUMSKA

CFSERVAT RR IJA

VCLGINA 7

11550 PEOGRAD

YUGCSL DVIA

TENCRIO-TAGLE G DR
ESC CEQN
CIE CHENA 23
CH-12II GENEVA 23
SWITERLA:VD

TERASHITA YOICHI PROF KANAZAWA INST TECHN

ACNCICH CHO

NINANIKYCKU

KANAZAWA 921

JAPAN

TERRIEN JEAN CIRECTEUR PAVILLON DE BRETEUIL F-923 IO SEVRES

FRARCE

TESKE RICHARD G PROF DEPT OF ASTRONOMY

UAIVERSITY OF

NICHIGAN

ANN ARBOR MI 48109

U.S.A.

THE PIK SIN

ASTRCNCMICAL INST

RCETERSSTRAAT IS

1018 WF AMSTERCAM

THE NETHERLANDS

THCNAS CAVID V CR

SCIENCE RES CQUNCIL

$P$ C EOX 18

SWINDON SN2 IET WILT

U.K.

THCNAS JOHN H PROF

CEPT MECH AERO SC

UNIVERS ITY ROCHESTER

RCCHESTER NY 14627

U. S.A.

THONASSCN PETER DR

$N R$ A L

JCCRELL BANK

NACCLESFIELD

CHESHIRE SKII 9DL

U.K.

THONPSCN LAIRD A CR

OEPT PHYSICS ASTRON

UAIV OF NEBRASKA

LINCOLN NE 68506

U.S.A.
TAYLOR KEITH DR

ROYAL GREENWICH

OBSERVATORY

HAILSHAM

EAST SUSSEX BN27 IRP

U.K.

TEHERANY D

83 AVENUE REY

TEHERAN

TEMESVARY S DR

R STRAUSSSTRASSE 127

D-8000 MUENCHEN 80

GERMANY, F.R.

TEPLITSKAYA R B DR

SIBIZMIR

664697 IRKUTSK 33

U.S.S.R.

TEREAIZH VALERY YU DR CRIMEAN STATION DF

STERNBERG

ASTRONOMICAL INST

NAUCHNY CRIMEA 334413

U.S.S.R.

TERZAN AGOP DR

OBSERVATO IRE DE LYON

F-69230 SAINT GENIS-

LAVAL

TEXEREAU JEAN M

CERGA

ÁVENUE COPERNIC

F- 06130 GRASSE

FRANCE

THERNOE KARL-AUGUST

TEKTOR

VINKELVEJ 36

DK- 2800 LYNGBY

DENMARK

THOMAS HANS-CHR ISTQPH DR MPI FUER PHYSIK UND

ASTROPHYSIK

FOEHR INGER RING 6

D-80OO MUENCHEN 40

GERMANY, F.R.

THOMAS RICHARD N DR

LAT

98 BIS RLVD ARAGO

FRANCE

THOMPSON A RICHARD DR

NAT RADIO ASTRO OBS

VLA PROJECT

SOCORRE NM 87801

U.S.A.

THOMPSON ROGER I PROF STEWARD QBSERVATORY UN IVER SITY

OF AR I ZONA

TUCSON AZ 85721

U.S.A.
TECH JACK L DR B 306 METROLLOY RLOG STANDARDS WASHINGTON OC 20234 U.S.A.

TEJFEL VIKTOR G DR $\angle A B$ DF LUNAR AND ASTROPHYSICAL INST 480068 ALMA-ATA U.S.S.R.

TEMPESTI PIERO PROF OSSERVATORI D ASTRONO MICO DI COLLURANIA ITG41 DO TERAMO

TER HAAR DIRK MAGDALEN COLLEGE
OXFORD OXI 4 AUU

$U . K$.

TERRAZAS MR L.R. INST NAC DE ASTROFIS OPI Y ELECTRONICA APT POSTALES $216 \quad 51$ PUEBLA

TERZIAN YERVANT PROF SPACE SCIENCES BLDG CORNELL UNIVERS ITY I THACA NY 14853

U.S.A.

THADDEUS PATRICK PROF INSTI TUTE FOR

SPACE STUDIES

2880 BROADWAY

NEW YORK NY 10025

U. S.A.

THIRY YVES R PROF

UNI VERSITE DE PARIS

VI TOUR 66

4 PLACE JUSSIEU

F-75230 PARIS CDX O5

FRANCE

THOMAS JOHN A OR

PHYSICS RAAF DEPT

UNIV OF MELBOURNE

PARKVILLE VIC 3052

AUSTRÁLIA

THOMAS ROGER J DR

CODE 682

NASA GODOARD SPACE

FLIGHT CENTER

GREENBELT MD 20771

U.S.A.

THOMPSON G I DR

ROYAL OBSERVATORY

EDINBURGH EH9 $3 \mathrm{HJ}$

U. K.

THOMSEN BJARNE B PROF INSY OF ASTRONOMY UNI VERSI TY OF AARHUS DK-80OO AARHUS

DENMARK 
THCNIVARD NORBERT DR CARNEGIE INST WASH $524 I$ BROAR BR RC NW
WASHINGTON DC 26015 U.S.A.

THUAN TRINH X DE

UCIV OF VIRGINIA

CFAKLOTTESVL VAZ2903

U.S.A.

TINCTHY J GETHYN CR

LAH FDR ATMOSPHER IC

UAIV OF COLORACO

BCULDER CO 803.0

U.S.A.

TIASLEY BLATRICE M PROF

YALE UNIVERSITY OBS

$C C X 2 \geq 23$

YALE STATION

NEW HAVEN CT 06520

U.S.A.

TLAMICHA ANTOVIN DR

ASTRE VOM ICAL

CESERVATORY

25165 ONDREJOV

CZECHUSLOVAK I A

TCLEEKT CHARLES R OR

S[X 3818

UNIV STATION

CHARLOTTESVL VA22901

U.S.A.

I GMHAUGH CLYOE W PROF

DEPT OF ASTRONOMY

FiCX 4520

NF STATE UNIVERSITY

LAS CRUCES NM $88 C 113$

U.S.A.

TCCNRE ALAN DR

NIT

77 MASSACHUSETTS AVE

CANGRICGE MA O2139

U.S.A.

ICRAL MASAHISA PROF

IEKYO SCIENCE UNIV

$1-3$ KAGURAZAKA

SR IN JYUKU

TCKYC 162

JAPAN

TCRRES CARLOS ALBERTO IR CES NACIONAL

K GENERAL RRUCE 586

$20 O \bar{J}$ RIO OE JĀNEIRO QRALIL

TCRROJA J PROF

CATECKA CE ASTRON

FACULTAD DE CIENCIAS

UAIV CCMPLUTENSE

SPAI

TCUSCY RICHARE DR

CCCE 7140

NAVAL RESEARCH LAB

WASHIVGTON CC 20375

U.S.A.
THOREN VICTOR E PROF 130 GOODBOOY HALL BLOONINGTON IN 47401

U.S.A.

TIFFT WILLIAM G PROF STEWARD DB SERVATORY

UNIVER S I TY

OF AR IZONA

TUC SON AZ 85721

U.S.A.

TINBERGEN JAAP DR

POSTBUS 9513

2300 RA LEIDEN

THE NETHERLANDS

TIUR I MARTTI PRO HELS INKI UNIV TECHN RADIO LABORA

OTAKAARI 5 A

SF-O2150 ESPOO 15

F INLAND

TODORAN IOAN DR

ASTRONOMICAL

OBSER VATORY

STR REPUBLICII 109

$3400^{\circ}$ CLUJ-NAPOCA

ROUMAN IA

TOMASI PAOLD DR

LAB RADIOASTRONOMIA

IIA IRNER ID 46

I-40126 BOLOGNA

TOM ITA KENJI PROF

RESEARCH INSTI TUTE F THEORETICAL PHYSICS TIROSHIMA UN

JAPAN

TOOMRE JUR I

DEPT ASTRO-GEOPHY-

UN IV OF COLORADO

BOULDER CD 80309

U.S.A.

TORELL $1 M$ DR

VSIA ASTRQN PMICD

J TALY

TORRES CARLOS DR

OBS ASTRON NACIONAL

UN IVER SIDAD DE CHILE

CASILLA 36-0

SANTIAGO

CHILE

TOSA MAKOTO DR NAGOYA UNIVERSITY FURUCHO CHIGUSA

NAGOYA 464

JAPAN

TOVMASSIAN H M DR BYURAKAN ASTROPHYSI CAL OBSERVATORY 378433 ARMEN IA

U.S.S.R.
THORNE KIP S PROF $130-33$

PASADENA CA 91125

U.S.A.

TIFREA EMILIA DR

OBS DE BUCAREST

STR CUTITUL DE

ARGINT

75212 BUCAREST

ROUMANIA

TING YEOU-TSWEN

ASTRONOMY SECTION

CENTRAL WEATHER BUR

64 KUNG YUEN ROAD

TAIPE I 100

TAIWAN

TJIN-A-DJIE HERMAN R E CR A STRONOMICAL INST

UNI VERSITY AMSTERDAM

ROE IER S STRAAT 15

AMSTERDAM 1004

THE NETHERLANDS

TOFANI GIANNI PROF

OSSERVATOR I Q ARCETRI

LARGO FERMI 5

I 50125 FIRENZE

TOMASKO MARTIN G DR

LUNAR PLANETARY LAB

UNIV OF ARIZONA.

SPACE SCIENCES BLDG

TUC SON AZ B5721

U.S.A.

TOMITA KOICHIRO MR

TOKYO ASTRONOMICAL

OBSERVATORY

OSAWA MI TAKA

TOKYO 181

JAPAN

TOPAKTAS LATIF A DR

UNI VERSITY

OBSERVATORY

UNI VERSITY ISTANBUL

TURKEY

TOROSHLIDZE TEIMURAZ I DR

ABASTUMANI ASTRO-

383762 GEORGIA

U. S. S.R.

TORRES PEIMBERT SILVIA CR

INST DE ÁSTRONOMI A

$\triangle P D O$ POSTAL 70264

MEXICO $20 \mathrm{D} \mathrm{F}$

MEXICO

TOSHIAKI MASUURA OSCAR DR

RUA I TACEMA

$\triangle P$ TO 43

SAO PAULO CEP 04530

BRAZIL

TOWNES CHARLES HARD DR

DEPT DF PHYSICS

RM 557 BIRGE HALL

RERKELEY CA 94720

U.S.A. 
TCZER OAVID C ER UAIVERSITY NEWCASTLE NLWCASTLE UPON TYNE NEI 7 RंU

U.K.

TRAVIIVG GERHARD PROF IAST ITUT FUER THEORE I ISCHE ASTROPHYSIK NEUEVHE INEQ FELC 294 D-t9 HEIDELAERG GFRNANY, F.R.

TREF LGER CHARLES F DR ASTRCN INST BASEL VENUSSTRASSE 7 CF-4IJ2 BINNINGEN SWITLERLAND

TREMKO JOZEF OR

CESERATETORY ESO

$5596 \%$ TATRANSKA

LCNNICA

CZECHOSLOVAK IA

TKINFLE VIRGINIA L DR

CEPT OF PHYS ICS

UNIVERSITY OF CAL IF

IRVINE CA 92717

U.S.A.

TRCITSKY $V S$ PROF DR

RACICPHYS ICAL TTUTE

o. 36 GORKIJ

U.S.S.R.

TRURA JANES W JR

DEPT OF ASTRONOMY

UAIV OF ILLINOIS

CESERVATORY

UREAINA IL 61801

U.S.A.

TSAC N PROF

NE 47 SEC 3

HSINE-1 ROAO

TAIWAN

TSESEVICH V P PROF DR

CEESSA STATE UNIVER

C.SERVATORY

270014 OCESSA

U.S.S.R.

TSUEAKI TOK 10 PROF FDCULTY OF EDUCATION

SHIGA UNIVERSITY

ISHIYANAHIRAT SUCHO

CHTSU 520

JAPAN

TSUJI TAKASHI

TCKYO ASTRONOMICAL CESERVATCRY

MITAKA

TCKYC 181

J $\triangle P A N$

IUCKER ROY H

RCYAL GREENWICH

OESERVATORY

HAILSHAM

EAST SUSSEX BN27 IRP

U.K.
TRAFTON LAURENCE M DR' ASTRONOMY DEPT

UN IVERSITY OF TEXAS

AT AUSTIN

AUSTIN TX 78712

U.S.A.

TREDER H J PROF DR

ZNTR INST ASTROPHYSIK

STERNW BABEL SBERG

ROSA LUXEMBURGST ITA

DDR- $15 \% 2$ POTSDAM

$G .0 . R$.

TREHAN SUR INOAR $K$ PROF

DEPT OF MATHEMATICS

PAN JAB UNIVERSITY

CHANDIGARH 160014

INOIA

TREUMANN RUDOLF DR

HOCHSTRASSE 58

SWITZERLAND

TRITAKIS BASIL P DR ASTRONOMY APPL MATHS

ACADEMY OF ATHENS 14 ANAGNOSTOPOULOU ATHENS 136 GREECE

TRUEMPER JOACHIM PROF MPI FUER EXTRATERRESTR ISCHE PHYSIK D-8046 GARCHING BEI MUENCHEN

GERMANY, F.R .

TRUTSE YU L DR

INSTITUTE OF PHYSICS

USSR ACADEMY OF SCI

U.S.S.R.

TSAP T T DR

CR IMEAN ASTROPHYSI-

CAL OBSERVATORY USSR

ACAD OF SCI NAUCHNIY

334413 CR IMEA

$U \cdot S \cdot S \cdot R$.

TSIKOUDI VASSILIKI PH D DEPT OF ASTRONOMY

UNIV OF IOANNINA

IOANN INA

TSUBOKAWA IETSUNE DR INTERNATIONAL LATITU DE OBSER VATORY MIZUSAWA IWATE JAPAN

023

TSURUTA SACHIKO DR MP I PHYS UND ASTROPH FOEHR INGER R ING 6 POSTFACH 401212 D-8000 MUENCHEN 40 GERMANY, F.R.

TUCKER WALLACE H DR 保S CAMBR IDGE MA 02138 U.S.A.
TRAN MINH N DR

OBS DE MEUDON

F-92190 MEUDON

FRANCE

TREFFT 2 ELEONORE E DR MPI F PHYSIK UND

ASTROPHYSIK

FOEHRINGER RING 6.

D-8OOO MUENCHEN 40

TRELLIS MICHEL DR

OASERVATOIRE DE NICE

$B$ P 252

F-060D7 NICE CEDEX

TRE XLER JAMES H MR

5609 OTTAWA STREET

LABDRATORY

OXON HILL MO 20021

U.S.A.

TRITTON KEITH P DR

ROYAL OBS EDINBURGH

BLACKFORD HILL U.K.

TRULSEN JAN K PROF UNIVERSITY OF TROMSO $N-9001$ TROMSO NORWAY

TSAI CHANG-HSIEN DIRECTOR

GENERAL SECRETARY ASTRO SOCIETY CHINA

TAIPEI OSSERVATORY

TAI WAN

TSCHARNUTER WERNER $M$ DR

MPI FUER ASTROPHYSIK

FOEHRINGER RING 6

D-8000 MUENCHEN 40

GERMANY, F.R.

TSI QUMIS ALEXANDROS DR

DEPT OF GEODETIC

ASTRONOMY

UNIV OF THESSALONIKI

THESSALONIKI

GREECE

TSUCHIYA ATSUSHI DR PROF TOKYO ASTRONOMICAL OR SERVATORY

OSAWA

OSAWA MITAKA TOK 181 JAPAN

TSVETKOV TSVETAN DR

DEPT OF ASTRONOMY

FACULTY OF PHYS ICS

1126 SOF IA

BULGARIA

TUFEKCIOGLU ZEKI DR

ASTRQNOMY DEPT

UNIVIOF ANKARA

FEN FAKULTESI

ANKARA 
TULL RCRERT G

DEPT CF ASTRONOMY

WNIVERSITY OF TEXAS

AT AUSTIN RLM 15212

ALSTIV TX 78712

U.S. A.

TUCVIVTN ILKKA V CR

TAHT IT CRMINMAK

SF-0O130 HELSINK I 13

FINLANC

TUKALR RARRY E CRR

WRAC NTNT RD

CFARLUTTESVL VA22901

U.S.h.

TUKA K KENNETH C CR

T ICA OF WASHINGTON

5241 AREAD BR RE NW

WSHIUGTON OC 20015

U.S.A.

TURTLE $\triangle J$ JR

LEPT TIF PHYS ICS

UA IVEXS ITY OF SYCVEY

AUSTRALIA

IUZI KCNCSUKE TR

$-2 \overline{2}-\ddot{2}$ ?

TCKYE 181

JAPAY

TYSLIN JOHN A QR

QELL LABS RM 1D-316

GOJ NOUNTTAIN ÁVE

NURRAY HILL NJ 07974

U.S.A.

IJCHICA YUTAKA DR

TCKYO ASTRONOMICAL

CESEIVATORY

CSDW

NITAKA TCKYO 181

JAPAN

UESUGI AKIRA DR

CEFT CF ASTRONDMY

UNIVEKSITY OF KYOTO

SAKYUKU KYOTO 606

JAPAN

ULNSCHYEIDER PETER CR

IAST FUER ASTRONO

AN HUBLANO

C-BT:S WUERZ BURG

CERNAVY, F.R.

ULRICH ROGER K PROF

CEPT OF ASTRDNOMY

UAIV OF CALIFORNI

Q931 MSB

LCS AVGELES CA 90024

U.S.A.

UANC WASABURO PRCF

DEPT OF ASTRONOMY

UNIVERSITY OF TOKYO

EUlikYO-KU

TCKY 113

JAPAi
TULLY JOHN A DR

QBSERVATOIRE DE NICE

F- 60 O 7 NICE CEDEX

TUOMINEN JAAKKO $V$ PROF

PIHLA NATIE 49
SF-O B 20
FF

FINLANO

TURNER DAVID G DR

DAVID DUNLAP OBS

UNIV OF TORONTO

R. ICHMOND HL L4C 4 YO

CANAOA

TURNER MARTIN J L L DR

$X$ RAY ASTROND
PHYS ICS DEP

UN IV OF LEICESTER

LEICESTER LEI TRH

U.K.

TUTUKOV A $Y$ DR

A STRONOM ICAL COUNCIL

PYATNITSKAYA UL 48

109017 MOSCOW

U.S.S.R.

TWISS R Q DR

NATIONAL PHYSICAL

LABORATCRY

TEDDINGTON MIODLESEX

$U . K$.

TZVETKDV MILCHO K DR

BULGAR IAN ACADEMY

OF SCIENCES

ICOO SOFIA

BULGARIA

UDAL'TSOV $V$ A DR

PHYSICAL INST I TUTE

USSR ACADEMY OF

SC IENCES

117924 MOSCOW

ULFBECK OLE DR

NIELS BOHR INST

BLEGDAMSVEJ 17

DK - 2100 COPENHAGEN-D

DENMARK

ULRICH BRUCE T PROF

C $/ 0$ CERN

CH-1 121 GENEVA 23

SWITZERLAND

UNDERHILL ANNE B DR GODDARD SPACE FL CTR GREENBELT MD 20771 U.S.A.

UNSOEL D ALBRECHT PROF INST THEO PHY STERNW NEUE UNIV PHYSIK ZNT OLSHAUSENST GB N $61 \mathrm{C}$ $D-2300 \mathrm{KIEL} 1$ GERMANY, F.R.
TULLY RICHARD BRENT DR UNI VERS I TY OF HAWAI I 2680 WOODLAWN OR HONOLULU HI 96822

U.S.A.

TURLO ZYGMUNT OR

ASTRONOMICAL CENTER

UL CHOPINA I2-IB

POLAND

TURNER EDWIN L DR

PRINCETON UNIV OBS

PEYTON HALL 08540

U.S.A.

TURON PIERRE

INST D'ASTROPHYSIQUE

98 BIS BO ARAGO

$F-75014$ PARIS

TUVE MERLE A DR

5241 BROAD QRANCH RD

WASHING

TYLER JR G L DR

STANF ORD UNIV

STANF ORD CA 94305

U.S.A.

UCHIDA JUICHI DR

TOHOKU GAKUEN

UNI VERSITY

TAGAJYOMACHI

MIYAGI 985

JAPAN

UENO SUEO PROF

KANA ZAWA INSTITUTE

OF TECHNOLOGY

NONOI CHIMACHI

I SHIKAWA 921

JAPAN

ULMER MELVILLE P PROF DEARBORN OBSERVATORY

NORTHWESTERN

UNI VERSITY

EVANSTON IL 60201

U.S.A.

ULRICH MARIE HELENE D DR

ESO C O CERN

CH- 1211 GENEVA 23

SWI TZERLAND

UNDERWOOD JAMES H DR

INSTITUTE F PLASMA

RESEARCH

STANFORD UNI VERSITY

STANF QR

U. S.A.

UPGREN ARTHUR R DR

VAN VLECK

OBSERVATORY

WESLEYAN UNI VERS I TY

MIDDLE 
VAN DER KKUIT PIETER C DR KAPTEYN LABORATORIUM

PCSTEUS 800

GRCNINGEN

THE NETHERLANDS

VAN DIGGELEN J DR

STERREWACHT

ZCNNENEURG 2

3512 NL UTRECHT
THE NETHERLANDS

VAN FLANDERN THCNAS DR WS NAVAL DBSERVATORY

U.S.A.

VAN HERK-KLUYVER H A DR

KCRTE HAARDER 58
2415 AV NIEUWERBRUG

THE NETHERLANOS

VAA HOUTEN C J CR

STERREWACHT

PCSTUUS 9513

2300 RA LEIDEN

VAN LEER B DR

PCSTRUS 951

2300 RA LEICEN

THE NETHERLANDS

VAN REGEMORTER HENRI DR OESERVATOIRE

DE NEUDCN

$F-9219 G$ MEUDON

FRANCE

VAN SPEYRROECK LEON P DR

CTR TUR ASTROPHYSICS

6) GAZDEN ST

CANERIEGE MA O2138

U.S.A.

VAN WOERDEN HUGO DR

K.AFTEYN LABORATORIUM

PCST CUS 800

TREA INGEN

VANCERVCORT PETER O OR

DEPT ASTRON ASTROPH

$1100-14$ E 58TH ST

CHICAGC IL 60037

U.S.A.

VARCANIAN R A OR

EYUPAKAN ASTROPHYSI-

CAL CBSSERVATORY

378433 ARMENIA

U.S.S.R.

VARSHALOV ICH D A OR

PHYS IKOT-TECHNICAL

IAST ITUTE USSR

194021 LENINGRAD

U.S.S.R.
VAN DER LAAN H PROF DR

STERREWACHT

POSTBUS 9513

2300 RA LEIDEN

VAN DORN BRADT H DR

MIT RM 37-581

CTR FOR SPACE RES

CAMBR IOGE MA O2139

U.S.A.

VAN GENDEREN A.M. DR .

STERRENWACHT LEIDEN

POSTBUS 9513

2300 RÁ LEIDEN

VAN HOOF A PROF EM

A STRONOMI SCH

INSTITUUT KU LEUVEN

NAAMSESTRAAT 61

$B-3000$ LEUVEN

BELGIUM

VAN HOUTEN-GROENE VELD

INGR ID DR

STERREWACHT LEIDEN

POSTBUS 9513

2300 RA LE IDEN

VAN NIEUWKOOP J DR IR

STERREWACHT

SONNENBORGH

ZONNENEURG 2

3512 WK UTRECHT

VAN RENSBERGEN WALTER DR

ASTROPHYSISCH INST

VRIJE UNIVERSI TEIT

PLEINLAAN 2

$B-1050$ BRUSSEL

BELGIUM

VAN $T$ VEER FRANK DR

INST D ASTROPHYSIQUE

Q $B$ IS BLVD ARAGO

F-75014 PARIS

FRANCE

VANDEN BOUT PAUL A

ASTRONOMY DEPT

RLM IVERSIIY OF TEXAS

AUSTIN TX 78712

U.S.A.

VANYSEK VLADIMIR PROF

ASTRONOMY DEPT

CHARLES UNIVERSITY

SVEOSKA $B$

150 OO PRAHA 5

CZECHOSLOVAKIÁ

VARDYA M S OR

TATA INSTITUTE OF

FUNDAMENTAL RESEARCH

HOMI BHABHA RD

BOMBAY 400 DO5

INDIA

VASHKOV'YAK S N. DR STERNBERG STATE ASTRONOMICAL INSTITUTE 117234 MOSCOW

U.S.S.R.
VAN DESSEL EDWIN LUDO DR KON STERRENWACHT

RINGLAAN 3

B-1180 BRUSSELS

BELG I UM

VAN DUINEN R DR SPACE RESEARCH DEPT

$P$ BOX 800

GRONINGEN

THE NETHERLANDS

VAN HERK G

STERREWACHT VAN

LEIDEN

POSTBUS 9513

2300 RA LEIDEN

THE NETHERLANDS

VAN HORN HUGH M PROF

DEPT PHYSICS AND

ASTRONOMY

UNI VERSITY ROCHESTER

ROCHESTER NY 14627

U.S.A.

VAN HOVEN GERARD DR

DEPT OF PHYSICS

UNIV OF CALIFORNIA

IRVINE CA 92717

U.S.A.

VAN PARADIJS J DR

ASTRONOMICAL INST

ROETERSSTRAAT 15

AMSTERDAM IOI8

THE NETHERLANDS

VAN RIPER KENNETH A DR

DEPT OF PHYSICS

UNIV OF ILLINOIS

URBANA

VAN T VEER - MENNERET

CLAUDE DR

98 BIS BLVD ARAGO

F-75014 PARIS

FRANCE

VANDERLINDEN HENRI L PROF UNI VERSI TE IT GENT

B-IITO RRUSSEL

BELGIUM

VAPILLON LOIC J DR

PARI S-MEUDON

F-92 190 MEUDON

FRANCE

VARSAVSKY C M DR

BUENOS AIRES

ARGENTINA

VASILEV VLADIMIR M DR

PULKOVO OBSERVATORY

196140 LENINGRAD

U.S.S.R. 


\section{VASILEVA GALINA J DR PULKEVC OBSERVATORY $19614 \%$ LENINCRAD \\ U.S.S.R.}

VALCLAIR SYLVIE C DR CASERVATOIRE DE F -92190 MEUDON

FRANCE

VEELER GLENN J CR 4800 OAK GROVE ORIVE PASACENA CA 91103 U.S.A.

VELSHE ALBERT G PROF DR OESERVATOIR AVENUE CIRCULAIRE R-I190 BRUSSELS PEL'GIUM

VENUGOPAL $\checkmark R$ RR

FACIC AST RONOMY

CENTRE OF IIFR

PCST BOX 8

CETACAMUINO 64300$]$

INCIA

VERGNANO A PROF

CSS ASTRONOMICO

ION 25 PIND TORINESE

I TALY

VERCN PHILIPPE CR

CESERVATOIRE

CE NEUCCN

F-92195 MEUDON

FRANCE

VETESNIK MIROSLAV DR DEPT OF ASTRONOMY PURKYNE UNIVERSITY KCTLARSKA 2

61137 BRNO

CZECHOSLOVAK IA

VICAL J L DR

CES DU PIC-DU-MILI BIGQRRE

FRANCE

VIGIER JEAN-PIERRE DR .

INST ITUT H.POINCARE

$\frac{1}{F}=75005$ PARIS

FRANCE

VILA SAMUEL C PROF

DEPT OF ASTRONOMY

UNIVERS ITY OF PENNA

PHILACELPHIA PAI9104

U.S.A.

VINER MELVYN R DR

ASTRCNCMY GROUP

CUEEN'S UNIVERSITY

KINGSTCN ONT KTL 3NG

CDNADA

\section{VASILEVSKIS STANISLAUS \\ 455 GRAND AVE NO 16 \\ PÁLD ALTO CA 94306 \\ U.S.A. \\ VAUGHAN ARTHUR HDR \\ HALE OBSERVATORIES \\ PASADENA CA 91101 \\ U.S.A. \\ VEIS GEORGE PH D \\ NAT TECHNICAL UNIV \\ ATHENS}

VELTMANN ULO ILMAR OR

TARTU CIB SER VATORY

202444 ESTONIA

U.S.S.R.

VERBEEK PAUL DR

GEORGE MINNELAAN 50 9830 S MARTENS-LATEM

BELGIUM

VERN I ANI FRANCO PROF

CNR METEOR PHYSICS I-40126 BOLOGNA

ITALY

VERSCHUUR GERRIT L PROF UNIVERSITY OF

COLORADO

BOULDER CO 80302

U.S.A.

VEVERKA JOSEPH DR

312 SPACE SC BLDG

CORNELL UNIVER SI

U.S.A.

VIDAL $N \vee D R$

DEPT OF PHYSICS AND

ASTRONOMY

TEL - AVIV UNIVERSITY

TEL-AVIV

I SRAEL

VIGOTTI MAR IO

LABORATOR IO DI

RADIOASTRONOMIA

VIA IRNER IO 46

$I=40126$ BOLOGNA

VILHENA DE MORAES R DR DEPTO DE ASTRONOMIA ITA-CTA 200 SAO JOSE DOS I 2200 SAO JOSE DOS BRAZ IL

VINTI JOHN P DR

RM W91-202

MEASUREMENT SYST LAB

CAMBR IOGE MA 02139

U.S.A.
VAUCLAIR GERARD P DR

OBSERVATOIRE DE

PARI S-MEUDON

$F-92190$ MEUDON

FRANCE

VAZQUEZ MANUEL DR

INST ASTROFISICA

DE CANARIAS

CANARIAS

SPAIN

VE I SMANN UNO DR TARTU OBSERVATORY 202444 ESTONIA U. S. S.R.

VELUSAMY T DR RÁDIO ASTRONOMY CTR P O BOX NO 8 OOTACAMUND 643001 INDIA

VERDET JEAN-PIERRE DR OBSERVATOIRE

DE PARIS 61 AVE DBSERVATOIRE FRT5014 PARIS

VERON MARIE-RAULE DR

OBSERVATOI RE DE

MEUDON

F-92190 MEUDON

VESECKY J F DR

TEP ASTRONOMY

LEICESTER LEI $7 R H$

U. K.

VICENTE RAIMUNDO D PROF R MESTRE AVIZ $30 \mathrm{R}$ C PORTUGALISBOA.

VIDAL-MADJAR ALFRED DR BP 10 F-91370 VERRIERES LE BUISSCE

VIIK TONU $D R$

TORAVERE OBS

202444 TARTU RAJ EST

U.S.S.R.

VILHU OSMI DR

ORS AND ASTROPHY LAB

UNIV OF HELSINK

TAHTI TORNI NMAKI

SF-OOI30 HELSINKI 13

FINLAND

VIOTTI ROBERTO DR

CNR ASTROF I S I CA

I-00044 FRASCAT

ROMA

I TALY 
$\checkmark I R G O P I A N D R$

CSS ASTRENOMICD

VIA TRICNFALE 204

IT 136 ROMA

VITCN NAURICE DR

ALLESE PEIRESC

FRANCE

\section{VCELK HEINRICH J PROF \\ MPI FUER KERNPHYSIK \\ PCSTFACH 103980 \\ D-69, 3 HE IDEL BERG \\ GERNANY, F.R.}

VCLLANE H DR

ASTRENOMISCHES INST

DER UVIVERS ITAET

C-5 300 HON

GERNANY, F.R.

VCN DER HEIDE JOHANN DR $\triangle L A R$ UUSSTR I 2

C- 200 J HAMBURG 19

GERNANY, F.R.

VCN WEIZSAECKER C F

PRCF DR

M $\triangle X-P L A N C K$ - INST I TUT

RIEMERSCHMID STR 7

$C-\sum 130$ STARNEERC

GERNANY, F.R.

VCRCSHILOV V I DR

OESERVATORY UKRAINI-

AN $\triangle C A D$ OF SCIENCES

$252127 \mathrm{KIEV}$

U.S.S.R.

VREUX JEAN MARIE CR

INST O ASTRUPHYS I QUE

UNIVERSITE DE LIEGE

EELGIUN

VUJNOVIC VLADIS DR

INSTITUTE OF PHYSICS

CF THE UN IVEPS ITY

F C $B 34$

41001 Z AGREB

YUGCSLAVIA

WACHNANN A A PROF DR

SCHNIEDESBERG 2

D-2i, 57 RE INREK

GER, NAVY, F.R.

WACE CAMPRELL M DR

NAT RACIO ASTRON OBS

$P C$ WOX O

SCCERRE NM 87801

U.S.A.

WAGA.ER WILLIAM J CR

HICF ALTITUDO

$P C$ BDX 3700

BCULEER CO 80307

$U . S . A$.
VISVANATHAN NATARAJAN DR

MT STROMLO

OBSER VATORY

WODEN PO ACT 2606

VIVES THEODORO JOSE DR

UFMG

UFNG

BRAZIL

VOGT NIKOLAUS OR

EUROPEAN SOUTHERN

OBSER VATORY

SCHLE ISSHE IMER STR 17

D-8046 GARCHING B M

GERMANY, $F \cdot R$.

VOLONTE SERGE DR

TORUE CAPOR

$B-7020$ HYON

BELGIUM

VON HOERNER SEBASTIAN DR

NAT IONAL RADIO ASTRO

NOMY OB SER VATORY

$P$ O BOX 2

GREEN BANK WV 24944

U.S.A.

VONDRAK JAN DR

ASTRONOMICAL INST

CSAV

BUDECSKA 6

12023 PRAHA 2

VORPAHL JOAN A DR

AEROSPACE

CORPORATION

PO BOX 92957

LOS ANGELES CA 90009

U.S.A.

VSEKHSVYATSKIJ SERGIUS $K$

PROF

KIEV ASTRONOMICAL

DBSER VATORY

$252053 \mathrm{KIEV}$ PER 3

U.S.S.R.

VUKICEVIC K M PROF DR

DEPT OF ASTRONOMY

FACULTY OF SCIENCES

STUDENTSK I TRG 16

11000 REOGRAD

YUGOSLAVIA

WACKERNAGEL H BEAT DR

2939 COUNTRY CLUB DR

COLORADO SPRINGS

CO 80909

U.S.A.

WAGMAN NICHOLAS E PROF

3 ALLEGHENY CTR

AP T 120

PITTSBURGH PA 15212

U.S.A.

WAGONER ROBERT $V$ PROF DEPT OF PHYSICS STANFORD UNIVERSITY STANFORD CA 94305

U.S.A.
VITINSKI J YUR I J I DR

PULKOVO OBSERVATORY

196140 LENINGGRA

U.S.S.R.

VLACHOS DEMETRIUS G PROF UNIV THESSALONIKI

DEPT OF GE RDES

GREECE

VOIGT HANS H PROF

UNI VER SI TAETS-

STERNWARTE

GEI SMARLAÑDSTR 11

D-3400 GOE TT INGEN

GERMANY, $F$. R.

VON BORZESZKOWSKI H H OR

DER ADW DER DDR

ROSA-LUXEMBURGST 17 A

DDR- 1502 POTSOAM

G.D.R.

VON SOCHER HERMANN DR

INST FUER ASTRONOMIE

DER UNIV WIEN

TUERKENSCHANZSTR 17

$A-I I B O$ WIEN

AUSTRIA

VORONTSOV-VEL'YAMINOV B A

PROF OR

STERNBERG STATE AS-

TRONOMICAL INSTITUTE

117234 MOSCOW

U.S.S.R.

VRBA FREDERICK J DR

S NAVAL OBS
O BOX 1149

FLAGSTAFF AZ 86002

U.S.A.

VUUREADR D LONG I TUDES

77 AVENUE DENFERT

ROCHEREAU

FT-75014 PARIS

FRANCE

VYALSHIN GENNADIJ F DR

MAIN ASTRONOMICAL OB

PULKOVO $M-140$

196140 LENINGRAD

U.S.S.R.

WADDINGTON C JAKE PROF

SCHOOL PHYS ASTRON

UNIV MI NNE SOTA

116 CHURCH ST S.E.

MINNEAPOLIS MN 55455

U.S.A.

WAGNER RAYMOND L DR

FORD AEROS PACE

2880 E.FOUNTAIN BLVD

$\mathrm{F}-10$

COLO SPGS CO 80910

U.S.A.

WAKAMATSU KEN-ICHI DR

JUNIOR TECHN COLLEGE

GIFU UNIVERSITY

KAGAMIGAHARA

GIFU 504

JAPAN 
WAKC KCJIRO DR

OE CUSERVATORY LATITU

NIZUSAWA IWATE 023

JAPAN

WALKER ARTHUR B C JR PROF

INST FCR PLASMA

RESEARCH

STANFOPC UNIVERS ITY

STAAFORO CA $943 C 5$

U.S.A.

WALKER MERLE F PROF

UAIVERSITY OF CAL IF

SANTA CRUZ CA 95064

U.S.A.

WALKER WILLIAM S G

14 APPLEYARD CRES

AUCKL AND 5

NEW ZEALAND

WALLACE PATR ICK T CR
ANGLC-AUSTRALIAN OBS

$P$ C ROX 296

EFPING NSW2121

AUSTRALIA

WALLIS MAX K. CR DEPT APP MATH ASTRON UA IVERS ITY COLLEGE $P C$ POX 78

CAREIFF CFI IXL

U. K.

WALSH CENNIS CR

NRAL

JCCCRELL BANK

NACCLESFIELD

CHESHIRE SKII 9DL

U. K.

WANPLER E JOSEPH PROF

LICK OBSERVATORY

UAIVERSITY OF CALIF

SANTA

WANIER PETER GREGORY DR

CALTECH

OWENS VLY RADID ORS

PASALENA CA 91125

U.S.A.

WARD WILLIAM R DR

$J F L$

$48 \% 0$ OAK GROVE DRIVE

PASALENA CA 91103

U.S.A.

WARNAV J DR

INST DI ASTRONOM IA

UNAM

APT POSTAL 7D-64

NEXICC DF

MEXICO

WARNER PETER J CR

CAVENDISH LABS

NACINGLEY ROAD

CANBRINGE CB3 DHE

U.K.
WALBORN NOLAN R DR CERRO TOLOLO INTERAMER ICAN OBSER VA TORY

CASILLA 63 D

LA SERENA

CHILE

WALKER EDWARD $N$ MR

ROYAL GREENWICH OBS

HER STMONCEUX CASTLE

HA IL SHAM

E SUSSEX BN27 IRP

U.K.

WALKER R RL VET OERY

FLAGSTAFF STATION

BOX 1149

FLAGSTAFF AZ 86002

U.S.A.

WALL JASPER $Y$ DR

MULLARD RADIO ASTRO-

OBS CAVENOISH LAB

MADINGLEY RD

CAMBR IDGE CB 3 OHE

U.K.

WALLENQUIST AAKE A E PROF NORRLANDSGATAN $34 \mathrm{D}$

$5-75229$ UPPSALA

SWEDEN

WALMSLEY C MALCOLM DR

MPI FUER RADID-

ASTRONOMIE

AUF DEM HUEGEL 69

Q-530O BONN 1

WALTER HANS G OR

ASTRONOMISCHES

RECHEN-INSTI TUT

MONCHHOF STER $12-14$

D-6900 HE IDELBERG 1

GERMANY, F.R.

WAMSTEKER W DR

EUROPEAN SOUTHERN

OBSER VATORY

SCHLE I S SHE IMER STR 17

D- 8046 GARCHING

GERMANY, F.R.

WAPSTRA AALDERT H PROF

VAN WOENSEL KOOI J-

LAAN 48

NAARDEN

THE NE THERLANDS

WARDLE JOHN F C PROF BRANDE IS UNIVERSITY WAL THAM MA O2154

U.S.A.

WARNER BR IAN PROF

ASTRONOMY DEPT

UN IVER S ITY OF CAP

CAPE TOWN 7700

SOUTH AFR ICA

WARREN WAYNE H JR DR

NASA-GSFC

CODE 60I

GREENBELT MD 20771

U.S.A.
WALOMEIER MAX PROF

SWISS FEDERAL OBS

SCHMEL ZBERGSTR 25

CH-8006 ZUERICH

WALKER GORDON A H PROF

INST ASTRON SPACE SC

UNI VERS ITY OF $B$

2075 WESBROOK PLACE

CANADA

WALKER ROBERT M A PROF

PHYSICS DEPT

$B O X 1105$

WASHINGTON UNIV

ST LOUIS MO 63130

U.S.A.

WALLACE LLOYD $V$ DR

KITT PEAK NATI DNAL

DBSERVATORY

$P$ BOX 26732

TUC SON AZ 85726

U. S.A.

WALLERSTEIN GEORGE PROF

ASTRONOMY DEPT FM 20

UNIVERSITY OF

WASH I NGTON

SEATTLE WA 98195

U.S.A.

WALRAVEN TH DR

LEIDEN SOUTHERN

STATION P O BOX 13

BROEDERSTROOM

TRANSVAAL O240

SOUTH AFRICA

WALTER KURT PRDF DR

ASTRONOMI SCHES INST I

TUT DER UNIVERS ITAET

WALDHAUSERSTR 64

D-7400 TUEBINGEN

GERMANY, F.R.

WANAS M I DR

FACULTY OF SCIENCE

CAIRO UNIVERSITY

ARAB REP: OF EGYPT

WARD RICHARD A DR

W K KELLOGG RADIAT

LAB 106-38

CALTECH

PASADENA CA 91125

U.S.A.

WARES GORDON W OR

WEST NEWTON MA 02165

U.S.A.

WARNER JOHN W DR

NASA CODE TA-OI

MARSHALL SFC AL35812

U.S.A.

WARWICK JAMES W DR

ASTRO-GEDPHYSICS

DEPT

UNIV OF COLORADO

BOULDER CO 80309

U.S.A. 
WARWICK ROBERT S CR

UAIV OF LEICESTER

LEICESTER LEI 7RH

U.K.

WASSERMAN LAWRENCE H CR

LCWELL OBS

FLAGSTAFF AZ 86002

U.S. 1 .

WATERFIELD REGINALD L OR WCOLSTON OASERVATURY

NCRTH CADBURY NR

YECVIL SOMERSET

U.K.

\begin{tabular}{|c|}
\hline $\begin{array}{l}\text { WATTENEERG D PRCF } \\
\text { ARCH VHOLC STERNW } \\
\text { ALT TREPTOW I } \\
\text { CLR I IS3 BERLIN- } \\
\text { TREPTOW } \\
\text { C.C.K. }\end{array}$ \\
\hline $\begin{array}{l}\text { WEAVER THOMAS A OR } \\
\text { PHYSICS DEPT L } 372 \\
\text { LAVREVCE LIVERM LAB } \\
\text { PCESX BOQ } \\
\text { LIVERMCRE CA } 94550 \\
\text { U.S.A. }\end{array}$ \\
\hline 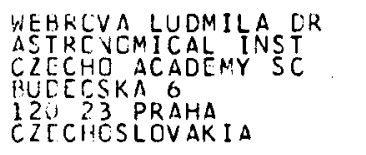 \\
\hline
\end{tabular}

WEELNAN DANIEL $W$ ASS PROF CYER CESERVATORY
VANCERBILT UNIV

CCX 13.33

NASHVILLE TN 37235

U.S.A.

WE EHLAU AMEL $1 A$ CR

ASTRCNCMY DEPT

UR IVERSITY DF

WESTERN OUTARIO

LCNLCN CNT NGA $5 B 9$

CARALA

WEICENANN VOLKER PROF

INST THEO PHY STERNW

CUELL UNIV PHYSIK ZNT

CLSHAUSENST GB N $6 I C$

T- $=3 \% \mathrm{~K}$ K I L I

GERN, A YY, F.R.

WILER KURT W CR

$N$ P I FUER RADIO-

ASTRCVOMIE

AUF LEN IIJECEL 69

O-53 0 BOHN 1

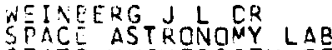

STATE UNIVERSITY DF

$\because Y Y$ AT $\triangle L B A N Y$

ALIANY NY 12203

i). 5.4 .

WEISS NIGEL O CR

LANPT SILVER STREET

CANEFICGE CB3 OEW

U.K.
WARZEE J DR

BIS RUE MATTOT

BELGIUM

WAS SON JOHN T UNIVERSITY OF CALIF

LOS ANGELES CA 90024

U.S.A.

WATERWORTH MICHAEL DR

UNIVERSITY TASMANIA

GPO BOX $252 \mathrm{C}$

HOBART 7001

AUSTRALIA

WAYMAN PATRICK A PROF

DUNSINK OESERVATORY

CASTLEKNOCK

CO DÚBLIN

WEBBER JOHN C DR

UN IVERSITY OF I LLI -

NOI IS OBS SER VATORY

URANA IL 61801

U.S.A.

WEBSTER ADRIAN $S$ DR

14 CLARE HALL

HERSCHEL ROAD

CAMBR IDGE

U.K.

WEEKES TREVOR C DR

MOUNT HOPKINS

OBSERVATORY

PMADO AZ 875640

U.S.A.

WEHLAU WILLIAM H PROF

ASTRONOMY DEPT

UNIVERSITY OF

WESTERN ONTARIO

LONDON ONT NEA 5B9

CANADA

WEIGERT ALFRED PROF HAMBURGER STERNWARTE GOJENBERG SWEG 112

GERMANY, F.R.

WEILL GILBERT M DR CENIRE NATIONAL

D ETUDES SPATIALES 129 RUE L UNIVE FRANCE

WEISHEIT JON C OR PRINCETON UNIV OBS PRINCETON NJ 08540 U.S.A.

WEISS WERNER W OR INST FUR ASTRON DER UNIV WIEN

TUERKENSCHANZSTR 17 $A-1180$ WIEN
WASHIMI HARUICHI DR RESEARCH INSTITUTE OF ATMOSPHERICS NAGOYA UNIVERSITY TOYOKAWA AICHI 442 JAPAN

WATANABE TAKASHI DR RESEARCH INSTITUTE OF ATMOPHER I CS NAGOYA UNI VERSITY TOYOKAWA 442 JAPAN

WATSON WILLIAM D PROF PHYSICS DEPT

UNI VERSITY

OF ILLINOIS

URBANA IL 61801

U.S.A.

WEA VER HAROLD F PROF

DEPT OF ASTRONOMY

UNI VERSITY OF

CALIFORNIA

BERKELEY CA 94720

U.S.A.

WEBBINK RONALD F DR

DEPT OF ASTRONOMY

UNIV OF ILLINOIS

URBANA IL $6 I 801$

U.S.A.

WEBSTER B LOUISE DR

ANGLO AUSTRALIAN

OBSERVATORY

$P$ O BOX 296

EPPING NSW 2121

AUSTRALIA

WEHINGER PETER A DR ROYAL GREENWICH OBS HERSTMONCEUX CASTLE HAILSHAM

SUSSEX BN27 IRP

U. K.

WEHRSE RAINER DR

INSTI TUT F THEORET ISCHE ASTROPHYSIK IM NEUENHE I MER F 294 D-6900 HEI DELBERG GERMANY, F.R.

WEI LER EDWARD J DR PRINCETON UNIV OBS PEYTON HALL PRINCETON NJ 08540

U.S.A.

WEIMER THEOPHILE P F UR CBSERVATOIRE

DE PARIS

61 AV L'OBSERVATOIRE F-75014 PARIS
FRANCE

WEISS EDWARD W DR

VAN VLECK OBS

WESLEYAN UNIV

MIODLETOWN CT 06457

U.S.A.

WEI SSKDPF MARTIN CH DR

NASAMMSC CODE ES-62

M.S.A. 
WE ISTRCP CONNA W CR

LAH FOR ASTRONOMY

$A \cap E$ SOLAR PHYS ICS

OREENIELT ME 20771

U.S.A.

WELIACHEW LEONIC CR

I RAN

53 FUT CES MARTYRS

FRANCE

WELLNANV PETER PFOF TR

IAST F ASTRON UND

ASTRIPPHS IK

SCHEIVER-STR

U-QOSO MUFNCHEN RO

GERNANY, F.R.

WENOKER HEINRICH J PROF

HANBURGER STERNWIRTF

CEJENHEROSWEG 112

C. 26.5$)$ HAMUURG 50

GERNANY, F.R.

WEALEL W UR

ZENTEALINSTITUT FUER

ASTRCPHYS IK

STERIWARTE SONNEPERG

CLE-64 SONNEBERG

G.L.?.

WEST RICHARD M ER

ESC CERN

CH-1 GENEVA 23

SWITZERLAND

WESTFOLO KEVIN C PROF

DEPT OF MATHEMATICS

NCNASH UNIVERSITY

CLAYTON VICT 3168

AUSTRALIA

WEYAAVN RAY J PEOF

UTEKARD QQSERVATORY

$A R I \angle C N A$

TUCSCN AL 85721

U.S.A.

WHELAI JOHN A JUCR

IAST OF ASTRONOMY

U.K.

WHITE NATHANIEL M DR

LCWELL OBS

FLAGSTAFF AZ 86002

U.S.A.

NHITE RAYMOND E CR

STEWARD OBSERVATCRY

UN IVERSITY OF

$A R I Z C V A$

TUCSCN AZ 85721

U.S.A.

WTITIVEY BALFOUR $S$

1102 E MISSOURI

ACRNAN OK 73069

U.S.A.
WELCH GARY A DR

DEPT OF ASTRONOMY

ST MARY'S UNIVERSITY

HAL IFAX NS B $3 H^{3} 33$

CANADA

WELLER CHARLES S DR

NAVAL RESEARCH

LAE CDDE 7124

WASHINGTON DC 20375

U.S.A.

WELLS CONALD C II I CR

IT PEAK NAT OBS

TUCSON AZ 85726

$U . S . A$.

WEN IGER SCHAME DR

OBSERVATOIKE

DE MEUDON

F-92190 MEUDON

FR ANCE

WESSELINK AORIAAN J DR 143 FALLS ROAD

BE THANY CT D6525

U.S.A.

WESTERHOUT GART DR

SCIENTIFIC DIREC TOR

US NAVAL OBSER VA TORY

WASHINGT

WESTPHAL JAMES A DR

CAL TECH

$170-25$
169 SOUTH MUDD

PASADENA CA 91125

U.S.A.

WHEELER J CRAIG

DSSPT OF ASTRONOMY

UNIVERSITY OF TEXAS

AUSTIN TX 78712

U.S.A.

WHIPPLE FRED L DR

CTR FOR ASTROPHYSICS

60 GARDEN ST

CAMBR IDGE MA 02138

U.S.A.

WHITE ORAN R DR

HIGH ALT I TUDE

OHSERVATORY

$B O X 3000$

BOULDER CO 80307

WHITEOAK J B DR

DSIV OF RADIOPHYSICS

P BOX 76

EPPING NSW 2121

AUSTRALIA

WHITNEY CHARLES A PROF PENTER FOR ASTRO-

60 GARDEN STREET

CAMBR IDGE MA 02138

U.S.A.
WELCH WILLIAM J PROF

UNIV OF CALIFORNIA

617 CAMPBELL HALL

BERKELEY CA 94720

U.S.A.

WELLGATE G BERNARD MR

CANEHEATH HOUSE

$\triangle R L I N G T O N$

POLEGATE

EAST SUSSEX BN26 $65 \mathrm{~J}$

U.K.

WEMPE J PROF DR

ZNTRINST ASTROPHYSIK

A STROPHYS DBS

TELEGRAFENBERC

DDR IS POTSDAM

G.D.R.

WENTZEL DONAT G DR

ASTRONOMY PROGRAM

UNI VERSITY MARYLAND

U.S.A.

WESSELIUS PAUL R DR

$P$ O HOX 800

GRONINGEN

THE NETHERLANOS

WESTERLUND BENGT E PROF

A STRONOMICAL

BOX 515

S-75 20 UPPSALA

WETHERILL GEORGE W W

CARNEGIE INST WASH

5241 BROAD BR RD NW

WASHINGTON DC 20015

U.S.A.

WHEELER JOHN A DR

DEPT OF PHYSICS

AUSTIN TX TB71

U.S.A.

WHI TAKER EWEN A

4332 E SIXTH STREET

TUCSON AL 85711

U.S.A.

WHITE R STEPHEN PROF

UNIVERSITY OF

CALIFORNI

RIVERSIDE CA 92521

U.S.A.

WHITFORD ALBERT E PROF

LICK OB SERVATORY

UNIVERSITY OF CALIF

SANTA CRUZ CA $\$ 5064$

U. S. A.

WHITROW GERALD JAMES PROF

41 HOME PARK RD

LONDON SW19 THS

U. K. 
WHITTET DOUGLAS C B DR UNIV CF LONOON ATCRY

NILL HILL PARK

LCNDON NWT 26 S

(I. $k$.

WIIING KENNETH G CR

CCOE 7144

US NAVAL RESEARCH

LAECPATCRY

WASHINGTON DC 20375

U.S.A.

WIEHR ERERHARC CR

UAIV STERNWARTE

GEIS VARLAND STR 11 .

LERM G G GOETT ING

WIESE WOLFGANG L ER

NATIONAL BUREAU OF

STANEAROS

WASHINGTON OC 20234

U.S.A.

WILCOX JUIHN M PROF

INSTITUTE FOR PLASMA

STANFO UNIVERSITY

U.S.A.

WILO PETEE AT ASSOC PROF

ASTRCANCMY DEPT

UAIVEZSITY

RCNCEBCSCH 7700

SCUTH AFRICA

WILKINS GEORGE A CR

CESERVATORY

HAILSHAM

EAST SUSSEX BN27 IRP

U.K.

WILLI INON RICHARE M

FERNAANK SCIENCE CTR

156 HEATOV PARK OR

ATLANTA GA 30307

U.S.A.

WILLIAYS DAVIO A CR

MATHEMATICS DEPT

UN IST

MANCHESTER MGO $10 \mathrm{C}$

U.K.

VILLIAMS JOHN A CR

PHYSICS DEPT

ALEICN COLLEGE

ALPICI

NILLIANS THEODORE B DR

129 PEYTON HALL

PRINCETCN NJ OB 543

U.S.A.

bI LLNORE A PETER PROF

SFAC RESEARCH CEPT

DC EOX 363

BJKMIVGHAM BIS 2TT

U. $k$.
WICKRAMASINGHE D T DR

INST I TUTE OF

ASTRONOMY

MADINGLEY ROAD

CAMBR IDGE CB 3 OHA

U.K.

WIDORN THOMAS OR

GRINZ INGERSTR 936

$A-1190$ WIEN

AUSTRIA

WIELEB INSK I RICHARD PROF

MP I FUER RADIO-

AS TRONOM IE

AUF DEM HUEGEL 69

O- 530 O BONN

WIETH KNUOSEN NIELS P DR SVEND TRDSTSVEJ 12 DENMARK

WILD JOHN PAUL DR CSIRO RADIOPHYSICS

2021

WILOEY ROGERT L PROF OR NORTHERN

ASTROPHYSICAL DBS

FLAGSTAFF AZ 86011

U.S.A.

WILKINSON PETER N DR

JOOR ELL RANK

MACCLESFIELD

CHESHIRE SKII 9DL

WILLIAMS CAROL A

ASSOC PROF

UNIVERSITY S FLORIDA

TAMPA FL 33620

U.S.A.

WILLIAMS IWAN P OR

APPL IED MATH DEPT

QUEEN MARY COLLEGE

LONDON EI 4 NS

U.K.

WILLIAMS PEREDUR M DR

UK INFRARED

TELESCQPE UNIT

9 CO LEILANI ST

HILOA. H

WILLIS A G DR

DEPT OF PHYSICS

BRANDE IS UNIVERSITY

WAL THAM MA O 2154

U.S.A.

WILLNER STEVEN PAUL DR

DEPT OF PHYSICS COII

UNIV OF CALIFOKNIA

SAN DIEGO

LA JOLLA CA 92093

U.S.A.
WICKRAMASI NGHE N C PROF

UNI VERSITY COLLEGE

DEPT OF APPLIED MATH

AND THEO PHYS

CARDIFF CFI IXL

U.K.

WIEDLING TORD DR

VILLAVAEGEN 15

S-6II OO NYKOEPING

WIELEN ROLAND PROF DR

TECH UNIV BERLIN

INST FUR ASTROPHYS

ERNST REUTER PLATZ 7

D 1000 BERLIN 10

GERMANY, F.R.

WILCOCK WILLIAM L PROF

SCHOOL PHY MOLEC SC

BANGOR GWYNEDD

LL57 2UW WALES

U.K.

WILD PAUL DR

ASTRONOMISCHES INST

UNIVERSITAET BERN

SIDLERSTR 5

CH-3O12 BERN

SWI TZERLAND

WILKENING LAUREL L DR

UNIV OF AR IZONA

TUC SON AZ 85721

U.S.A.

WILL CLIFFORD M DR

STANF ORD UNI

STANFORD CA 94305

U.S.A.

WILLIAMS D DR

RAOIO-ASTRONOMY LAB

UNIV DF CALIFORNIA

BERKELEY CA 94720

U.S.A.

WILLIAMS JAMES G DR

JPL $264-720$

4800 DAK GROVE DRIVE

PASADENA CA 91103

U. S.A.

WILLIAMS ROBERT E PROF STEWARD OBSERVATORY

UNI VER SITY OF

$\triangle R$ I ZONA

TUC SONAZ 85721

U.S.A.

WILLI S ALAN J DR

AND ASTRONOMY

UNIV COLLEGE LONOON

LONDON

U. K.

WILLS BEVERLEY J DR

REMT 15 OF 212

UNIVERSITY OF TEXAS

AUSTIN TX 78712

U.S.A. 
WLLS CEREK DR

UAIVCRSITY OF TEXAS

AUSTIN TX 78712

U.S.A.

WILSCN ALBERT G OR

STUDIES

PC ROX 113

TEPANGA CA 30290

U.S.A.

WISCN JANES R OR

LAWR LIVERMORE LAB

$L-35$

[IVERMORE CA 94550

U.S.A.

WILSCN PETER R PROF

TEPT OF APPLIEC

MATHEMATICS

UAIVERSITY OF SYONEY

SYLNEY 20137

LUSTRALIA

WILSCN RQBERT PRCF

DEPT PHYS ASTRONOMY

UAIV CCLLEGE LONDON

GCWER ST

LCADCN WCIE GBT

U.K.

WILSON W. J DR

UNIVERSITY OF TEXAS

CEPT OF ELECTRICAL

ENGINEER ING

AUSTIN TX 78712

U.S.A.

WIAK JOERN ERHARD DR

NPI FUER

RAC ICASTRONOMIE

AUF LEN HUEGEL 69

C-53) BONN

WINNEERG $\triangle N D E R S$ CR

MPI FLIER RADIO-

$\triangle S T R C N O M I E$

AUF DEN HUEGEL 69

D-5300 RONN

WITHEROE GEQRGE L CR

HARVARD COLLEGE OES

GO GARUEN ST
CANEKILGE MA O2138

U.S.A.

WITTNANIN AXEL CIETER PH D

UNIV STERNWART E

GE ISNARLANDSTR 11

CE-34OO GOETT ING
GERMANY, F.R.

WCEHL HUBERTUS DR

UNIVERSITAETS-

STERNWARTE

GEISNARLANDSTR 11

D-340O UOETT INGEN

GERMANY, F.R.

WCLFE ARTHUR M PROF

CEPT OF PHYSICS AND

ASTRCNCMY

UNIV OF PITTSEURGH

PITTSBURGH PA 15260

U.S.A.
WILLSON LEE ANNE OR E W F ICK OBSERVATORY PHYSICS

IOWA ŠT UNIVERSITY

AMES IA 50011

U.S.A.

WILSON ANOREW S DR UNIVERSITY OF SUSSEX

FALMER

BRIGHTON BNI $9 Q H$

U.K.

WILSON LIONEL OR

UNIT E S DEPT

LANCASTER UN IVERSITY

LANCASTER LAI $4 Y R$

U.K.

WILSON RAYMOND H DR

C $/ 0$ ANNIE WILSON

5829 NOR TH 25 TH ROAD

ARL INGTON VA 22207

U.S.A.

WILSON ROBERT WUR

BELL LABORATOR IES

HOH L 229

eOX $4 \mathrm{CO}$

HOLMDEL NJ 07733

U.S.A.

WINCKLER JOHN R PRDF

SCHOÓL OF PHYSICS

AND ASTRONOMY

UN IV OF MINNESUTA

MINNEAPOL IS MN 55455

$U \cdot S \cdot A$.

WINKLER FRANK P DR

DEPT OF PHYSICS

MIDOLEQURY COLLEGE

MIODLEBURY VT 05753

U.S.A.

WINNEWISSER GISBERT DR

MPI FUER RADIO-

A SIRONOMIE

AUF DEM HUEGEL 69

D- 5300 BONN

GERMANY, F.R.

WITT ADOLF N DR

RITTER ASTROPHYSICAL

RESEARCH CENTER

TOLEDO OH 43606

U.S.A.

WITZEL ARNO OR

MP I FUER RADIO-

ASTRONOM IEE

D- 5300 BONN

GERMANY, F.R.

WOLF BERNHARD PH D

MOMBER TSTRASSE 5
D-69OO HEIDELBERG

GERMANY, $F \cdot R$.

WOLFENDALE ARNULD W PROF PHYSICS DEPT

UN IVER SITY

SOUTH ROAD

DURHAM DHI 3LE

U.K.
WILLSTROP RODERICK $V$ DR

INST OF ASTRONOMY

MAD I NGLEY ROAD

CAMBRIDGE CB3 OHA

U.K.

WILSON BRIAN G PROF

SIMON FRASER

UNI VERSITY

BURNABY BC VSA IS6

CANADA

WILSON OLIN C

HALE OBSERVATORIES

813 SANTA BARBARA ST

PASADENA CA 91106

U.S.A.

WILSON ROBERT EE PROF

UNI VER S TY OF

SOUTH FLORIOA

TAMPA FL 33620

U.S.A.

WILSON THOMAS L OR MPI FUER RADIO-ASTRO

NOMIE

AUF DEM HUEGEL 69

D-530 RON RE

WING ROBERT F PROF

OHIO ST UNIVERSITY

174 WEST $18 \mathrm{TH}$ AVE

U.S.A.

WI NKLER GERNOT M R DR US NAVAL ORSERVATORY WASHING TON DC 20390

U.S.A.

WISNIEWSKI WIESLAW Z

LUNAR AND PLANETARY

LABORATORY

TUC SON AZ 85721

U.S.A.

WITTEN LOUIS PROF

DEPT OF PHYS I CS

UNI VERSI TY OF

CINCINNATI

CINCINNATI OH 45221

U.S.A.

WLERICK GERARD DR

QBSERVATOIRE PARIS

FRANCE

WOLF RAINER E A DR MPI FUER ASTRONOMIE KOENIGSTUHL D-6900 HEIDELBERG 1

WOLFF SIDNEY C DR

INSTITUTE

2680 WOODLAWN ORIVE

HONOLULU HI 96822

U. S. A. 
WCLSTENCROFT RAMQN C DR RCYAL CBSERVATCRY PLACKFORD HILL U.K.

WCCC F BRADSHAW PROF DEPT OF PHYSICS

AND ASTRCNOMY

GAIVERSITY FLORIDA U.S.A. WCCO JCHN A DR GL GAROEN ST CDNBRICGE MA 02138 U.S.A.

WCCOWARD $P R$ DR STERRCWACHT PCST UUS 9513 2300 RA LEIDEN THE NETHERLANDS

WECLLEY RICHARD SIR NAENOLIA HOUSE HAAKUAM NR PEVENSEY EAST SUSSEX

U.K.

WCROEN SINON P CK SACRAMENTO PEAK OBS SCLAR RES BRANCH SUNSPUT NM 88349 U.S.A.

VCSZCZYK ANDRZEJ CR

CHST OF ASTRONO

PIIOSTORUN

WRIGHT EDWARD L CR REPT OF PHY

NIT

CANBRIDGE MA U2139

U.S. A.

WR I GHT HELEN

579 FOREST LAKE CR

AACCVER NJ D7821

U.S.A.

WRIGHT NELVYN C H DR

RADIC ASTRONOMY

LAECRATORY

UAIVERSITY OF CAL IF

EERKEL

WU HSIN-HENG DR

NEPT CENTPHY UICS

CIUNG $-L$ l

TAIUAN

WYCKCFF SUSAN DR

PHYSICS DEPT

ARIZCNA STATE UNIV

$T E N P E A Z$ \& 5281

U.S. A.
WOLT JER LODEW I JK PROF. EUROPEAN SOUTHERN OBSERVATORY

CH-1211 GENEVA 23

WOOD HARLEY W DR

178 KENTHURST RD

KENTHURST 2154

AUSTRALIA

WOOD PETER R DR

MT STROMLO AND

PRIVATE BAG

WODEN A ${ }^{\circ}$ T 2606

WOOLF NEVILLE J TOR Y

UNIV OF ARIZONA

TUCSON AZ 85721

U.S.A.

WOOL SEY E G

695 BR IER WOOD

OTTAWA ONT

CANADA

WORLEY CHARLES E

ASTROMETRY AND ASTRO PHYSICS DIVISION

US NAVAL DBSER VATORY WASHINGTON DC 20390

U.S.A.

WRAY JAMESTDDR

UNIVERSITY OF TEXAS

AUSTIN TX $7871 \frac{1}{2}$

U.S.A.

WR IGHT FRANCES W DR

ASTRONOMY DEPT

HAR VARD COLLEGE

CAMBR IDGE MA 02138

U.S.A.

WRIGHT JAMES $P$ DR

DIV ASTRONOM ICAL SC

1800 G STREET N

WASHINGTON DC 20550

U.S.A.

WR I XON GEORGE T DR

DEPT OF ELECTR ICAL

ENG INEER ING

UN IVER S TY COLLEGE

CORK

IRELAND

WU SHI TSAN DR

SCHOOL SCIENCE ENGIN

UN IV OF ALABAMA

$P$ O BOX 1247

HUNTSVILLE AL 35801

U.S.A.

WYLLER ARNE A PROF

STOCK HOLM

S- 133 DO

SALTSJOEBADEN
WOOD DAVID B DR

WOOD ASSOCI ATES

BETHESDA MD 20014

U.S.A.

WOOD II I H J DR

UNIV OF VIRGINIA

CHARLOTTESVL VA22903

U. S. A.

WOOD ROGER DR

ROYAL GREENWICH OBS

HERSTMONCEUX CASTLE

HAI LSHAM

E SUSSEX BN27 IRP

WOOLFSON MICHAEL M PROF UNI VERSITY OF YORK YORK YOI $5 D D$

U.K.

WOOSLEY S E PROF

UNI VERSITY OF CALIF

SANTA CRUZ CA 95064

U.S.A.

WORRALL GORDON DR

RIRD SWOOD

EARDI SLEY HEREFDS

U.K.

WRIGHT ALAN E DR ANGLO-AUSTRALIAN OBS

P O BOX 296

EPPING N S W 2121

AUSTRALIA

WRIGHT GEOFFREY A E DR

INST F ASTROPHYS UND

EXTRATERR FORSCHUNG

AUF DEM HUEGEL 71

D-5300 RONN

WRIGHT KENNETH O DR DOMINION ASTROPHYSI CAL OBSERVATORY

$5071 W$ SAANICH RD

VICTORIA BC VBX $3 \times 3$

CANADA

WU CHI CHAO DR

COMPUTER SCIENCES CO

NASA GODDARD SPACE

FLIGT CNTR CODE 6B5

GREENBELT MD 20771

U.S.A.

WYATT STANLEY P JR PROF

DEPT GF ASTRONOMY

UNIV OF ILLINOIS

OBSERVATORY

URBANA IL 61801

U.S.A.

WYNN-WILLIAMS C G DR

INST FOR ASTRONOMY

UNI VERSITY OF HAWAI I

2680 WOCDLAWN DRIVE

HONOLULU HI 96822

U.S.A. 
NYYNE CHAZLES G PROF RCYAL GREENWICH

CFSERVATORY

HAILSHAN

EAST SUSSEX EN27 IRP

U.K.

YABUUT I KIYOSHI PROF

20 T ANAKA HIGASK I

HIVCYUCH MACHI

SAKYCKU 6:6 KYOTO

JAPAN

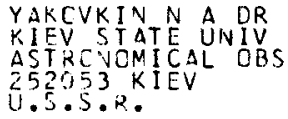

YANASHITA KOJUN DK

CEPT GF PHYSICS

DACOYA UNIVERSITY

FURCCIIC CHIKUSAKU

$\triangle A G C Y A 464$

JAPAN

YANKULLCVA IVANKA DR

DEPT OF ASTRONOMY

FACULTY OF PHYSICS

ANTCV IVAINOV STR 5

FULGARIA

YARCV-YARUVOJ $M$ DR

CF NYTU

IOTOS MOSCOW

U.S.S.R.

YAVNEL $\triangle L E X A N C E R$ A DR

NETEORITE COMMITTEE

UL UL I ANOVOJ $3 \mathrm{~K}$

I173:3 MOSCOW

U.S.S.R.

YECNANS DONALD K CR

JET PRCPULSIDN LAE

48 OAK GROVE CR

U.S.A.

YCKCYAMA KOICHI OR

INTERNATI ONAL LATITU

DE CUSERVATORY

NIZUSAWA IWATE 023

JAPAi:

YCRKE HAROLD W CF

UAIV STERNWARTE

GE ISNARLANDSTR 11

GERNAIYY, F.R.

YCSS KENNETH M CR

ASTRCNOMY DEP

UAIV CF ILLINOIS

CESEHVATOKY

UREANA IL 61801

U.S.A.

YCUAO LOUISE GRAY DR

1200 HCLLEMAN CR IVE

CELLEGE STATION

U.S.A.
XANTHAKIS JOHN N PROF

R C A A M A

I 4 ANAGNOSTOPOULOU

ATHENS 136

GREECE

YAHIL AMOS DR

ASTRONOMY PROGRAM

ESS BUILOING

SUNY AT STONY BROOK

STONY BROOK NY 11794

U.S.A.

YALLOP BERNARD D DR

ROYAL GREENWICH

DUSER VATORY

HAIL SHAM

EAST SUSSEX BN27 IRP

U.K.

YAMASHITA YASUMASA PROF TOKYO ASTRONOMICAL

OBSER VATORY

DSAWA

MITAKA TOKYO 181

JAPAN

YANOVITSKIJ EDGARDT G DR

MAIN ASTRONOMICAL OB

UKRAINIAN

252127 KIEV

U.S.S.R.

YASUDA HARUD PROF DR

TOKYO ASTRONOMICAL

ORSER VATORY

OSAWA

MITAKA TOKYO 181

JAPAN

YEIVIN Y PROF

TEL-AVIV UNIVERSITY

TEL-AVIV

Y ILMAZ FATMA DR

OQS DE LUNIVERSITE

I TANBUL

YONEYAMA TADAOKI DR

3-14-3-802

OHKUBO

SHINJUKU-KU

TOKYO 112

JAPAN

YOSHIDA JUNZO PROF

DEPT OF PHYSICS

KYOTO SANGYO UNIV

KAMIGAMO KITA-KU

KYOTO 603

JAPAN

YOUNG $\triangle N D R E W T$ TR

1200 HOLLEMAN DR

COLLEGE STATION

U.S.A.

YQUSEF SHAHINAZ M DR ASTRONOMY DEPT

FACULTY OF SCIENCE

CAIRO UN IVER SI TY

CAIRO

ARAB REP. OF EGYPT
YABUSHITA SHIN A PROF OEPT OF APPLIED MATH AND PHYSIC

KYOTO UNI VERSITY

SAKYOKU KYOTO 606

JAPAN

YAKHONTOYA N S DR

TICAL ASTRONOMY

192187 LENINGRAD

U.S.S.R.

YAMASAKI ATSUMA DR

DEPT EARTH SC ASTRON UNIVERSITY OF TOKYO KOMABA MEGURO-KU

TOKYO 153

JAPAN

YAMAZAKI AKIRA DR HYDROGRAPHIC DEPT TSUK I JI 5 CHUD KU TOKYO 104

JAPAN

YAPLEE B $S$

6105 WESTLAND DR

HYATTSVILLE MD 20782

U.S.A.

YATSKIV YA S DR

MAIN ASTRDNOMICAL

OBS UKRAINI AN ACADE-

MY OF SCIENCES

252127 KIEV

U.S.S.R.

YEN YUI - LIN PROF

DEPT OF ELECTRICAL

UNIVERSI TY TORONTO

ONT MSS 1 A4

YILMAZ NIHAL DR FACULTY OF SCIENCE DEPT OF ASTRONOMY

ANKARA

TURKEY

YORK DONALD G DR

PEYTON HALL

PR INCETON UNI VERS ITY

PRINCETON NJ 08540 U.S.A.

YOSHIMURA HIROKAZU DR DEPT OF ASTRONOMY

UNIVERSI AY OF TOKYO

YAYOI BUNKYO

TOKYO 113

JAPAN

YOUNG ARTHUR DR

A STRONOMY DEPT

SAN DIEGO STATE UNIV

SAN DIEGO CA 92180

U.S.A.

YOUSSEF NAHED H DR

ASTRONOMY DEPT

FACULTY OF SCIENCE

CAIRO UNIVERSITY

CAIRO REP. OF EGYPT 
YU KYUNG-LOH PRCF

SECUL AAT UNIVERS ITY

MININ-CCING

X.WANAK-KU

KEPULLIC OF KOREA

YUP1 S C.

TEYC UNIVEKSITY

HAKUSAN $5-28-20$

DUAKYC-KU

TCKYC II?

JAPAlv

ZACHELCV IGOR CR ASTRCVOMICAL INST CESERVAT CRY

251 CO CNOREJOV

CZLCHOSLOVAK IA

ZAITSEV VALERII VDR

ULYAYEVAST 46

6036,3 CCRKY

U.S.S.R.

ZAPPALA VINCENZS CR

CSSEQV

PIAC TORINESE

TCRINC

ZCANAVICIUS KAZIMERAS DR

ASIRCNC:MIJOS OBS

VILAIUS $3 I 0$

LITHUVIA

U.S.S.R.

ZEIPPEN C DR

F-9219O MEUDUN

FRAIC?

2ELENKA ANTOINE LR

ShISS FEDERAL

SCHNELZEERGSTR 25

CH-80O6 ?UER ICH

SHITLERLANAD

2. EVAKIN S $\triangle$ PROF TR

RADICPHYSICAL

RESEARCH INSTITUTE

$60360 \%$ GORKI

U.S.S.R.

ZIEPA STANISLAW OR

CBSEFIVATORIUM

DSTRCINMICZNE UJ

UL CRLA ITI

$34=24$ KRAKUW

ZINA RCBERT J DR

YALE UNIVERSITY

ICX ZU 23 YALE STAT

NEE HAVEN CT OG520

U.S.A.

ZIKIN HAPOLD DR

CAL TECH 264-33

PASALENA CA 91125

U.S.A.
YUAN CHI PRQF

DEPT OF PHYSICS $\mathrm{N}$

I 38 ST CONVENE AVE

NEW YUIRK NY IOOSI

U.S.A.

YUN HONG-SIK ASSOC PROF

SEOUL NAT UNIVERSITY

SIVLIM-DONG

KWANAK - K I

SEOUL 151

REPUEL IC OF KOREA

ZADUNAISKY PEDRO E PROF

AV MITRE 3 ICO

1663 SAN MIGUEL

BUENOS AIRE

ZANDER RODOLPHE OR

INST $D$ ASTROPHYSIQUE

UN IVERSI IE DE LIEGE

BELGIUN

ZARE K DR

DEPT OF MATHEMATICS

P BOX 6934

ISFAHAN

IP.AN

2EALY WILLIAM J DR

UK SCHMIDT TELESCOPE

PEIVATS BAG

COONBARABRAN

$N S W 2857$

AUSTRALIA

ZEL DOVICH YA B ACAD

SPACE RE SEARCH

INSTITUTE

USSR ACADEMY OF SCI

$117810 \mathrm{MO}$

ZELLNER BENJAMIN H DR

LUNAR AND PLANETARY

LABORATORY

UN IVERSITY ARI ZONA

TUCSON $A Z 85721$

U.S.A.

ZHCNGOLOVICH I D PROF DR

INSTITUTE OF THEORE-

TICAL ASTRONOMY

192187 LENINGRAD

U.S.S.R.

ZIKIDES MICHAEL C DR

PANEPISTIMIOPOLIS

ATHENS 621

GREECE

2 IOLKOWSKI J DR

COPERN ICUS ASTRONOMI

CAL CENTER

UL BARTYCKA 18

OO 716 WARSAW

PCLAND

Z IRKER JACK B OR

SACRAMENTÓ PEAK

OBSER VATORY

SUNSPCT NM 88349

U.S.A.
YUASA MANABU UR

DEPT OF ASTRONOMY

UNI VERSITY OF TOKYO

BUNKYO-KU

TOKYO 113

JAPAN

ZABRISKIE F R PROF

ALEXANDRIA PA 16611

U.S.A.

ZAHN JEAN-PAUL DR

OBSERVATOIRE DE. NICE

$B P 252$

F-06007 NICE CEDEX

FRANCE

ZAPPALA ROSAR10 ALDO DR

OSS ASTROF ISICD

CITTA UNIVERSITARIA

I-95125 CATANIA

I TALY

ZASOV ANATOLE V DR

CAL INSTITUTE

117234 MOSCOW

U.S.S.R.

ZEILIK MICHAEL II DR

DEPT PHYSICS ASTRCN

UNIV OF NEW MEXICO

BOO YALE BLVD BE

ALRUQUERQUE NM 87131

U.S.A.

2EL'MANOV A L CR

STERNBERG STATE AS-

TRONOMICAL INSTITUTE

117234 MOSCOW

U.S.S.R.

ZHELEZNIAKDV VLADIMIR V

PROF APPLIED PHYSICS

ULYANOOV STREET 46

603600 GORKIJ

U.S.S.R.

2IEBA ANDRZEJ PROF

ASTRONOMICAL

OBSERVATORY

UL ORLA ITI

$30-244$ KRAKOW

POLAND

2 IMMERMAN HELMUT DR

UNI VERS ITAETS

STERNWARTE JENA

SCHILLERGAESSCHEN 2

DDR-69 JENA

G.O.R.

ZIOLKOWSKI KRZYSZTOF DR

MICKIEWICZA 4 M 17

OI-5I7 WAR SZAWA

POLAÑ

ZLOBEC PAOLO DR

S LLRI ANA I A

I TALY 
2CNBECK MART IN V DR

CTR FCR ASTROPHYSICS

GC GARDEN ST 02138

U.S.A.

ZWAAA CORNELIS DR

UESEFVATORY

ZCNNENBURG 2

UTRECHT

THE NE:THERLANCS
ZUCKERMAN BEN M DR

ASTRONOMY PROGRAM

UN IVER SI TY MAR YLAND

COLLEGE PARK MO 20742

U.S.A.
ZVEREV MITROFAN S PROF DR PULKODVO OBSERVATORY 196140 LENINGRAD U. S. S.R. 